\title{
A SOCIO-CULTURAL ANALYSIS OF LEARNING ENGLISH IN UNASSISTED AND ASSISTED PEER GROUPS \\ AT UNIVERSITY IN VIETNAM
}

\author{
by
}

Lê Phạm Hoài Hương

\begin{abstract}
A thesis
submitted to the Victoria University of Wellington in fulfilment of the requirements for the degree of

Doctor of Philosophy

in Education
\end{abstract}

Victoria University of Wellington

2004 


\section{ABSTRACT}

The fieldwork for this study was carried out in Vietnam over a period of three months with a class of 45 first-year university students who were learning English as a foreign language. The conceptual framework was sociocultural theory as developed by Vygotsky and his followers. The focus was on small groups of peers as they practised speaking English. The processes involved in learning and using English were explored by studying what occurred within two kinds of groups. In one kind there were five firstyear students. In the second there were four first-year students and one fourth-year student. These are referred to as unassisted and assisted peer groups. Over the three months of the study all students in the class had an opportunity to work in an unassisted and an assisted group. Data were collected each week by audio- and video-recording an unassisted and an unassisted group discussing the same topic. The processes within each of the groups were compared on the basis of the social interaction and the use of classroom artifacts including the text book which supplied the topics for discussion. First-year students were interviewed following the classroom observations and they kept journals. Students reported their experiences of being assisted and unassisted and what they believed they had learnt from each. Information was also collected on support for learning the English language in the broader Vietnamese environment.

The results showed that the discourse pattern of the unassisted groups was unpredictable whereas in the assisted groups the senior student conducted a series of dyadic interactions with each student in turn. In both kinds of groups, students discussed the assigned topics but the assisted groups spoke almost entirely in English while the unassisted groups used more Vietnamese. Analysis of the incidence of Vietnamese showed the kind of situations which produced it. There were differences in 
the management of the tasks, and unassisted students had more trouble getting started. Observations showed that the unassisted students often teased others and laughed more often. The experience of speaking English amongst peers produced both stress and enjoyment irrespective of the type of group. Students from both groups reported that they had learned new words associated with the discussions of the topics set by the textbook.

The textbook was a major factor in guiding participation and structuring the continuity of the discourse. Unassisted students worked directly with the textbook whereas the senior student mediated the questions from the textbook. The textbook came from a foreign culture and the study illustrated how students used their knowledge of Vietnamese culture in giving responses to the questions in the text.

The study showed a complex mediation process consisting of interconnected layers. Mediation occurred both through the oral language of the discussions and through the written language in the textbook and on the blackboard, through the classroom teacher's instructions, by peers in both types of groups, and between the senior student and peers.

On the basis of the research findings recommendations are made for teaching practice in EFL classrooms. 


\section{ACKNOWLEDGEMENTS}

I would like to express my gratitude to those who helped shape this dissertation and whose encouragement led to its completion. I am sincerely thankful to my two supervisors: Dr Geraldine McDonald and Dr Val Podmore for their enthusiastic and insightful supervision throughout the research. Geraldine has given me input from her broad-based knowledge of sociocultural theory and by way of her professional perceptiveness. She has been a most important mentor to me. She gave me hours of time to reply promptly to my questions and to challenge me. Val has given me her organised vision of the research journey and of the structure of the thesis. I have learned from her expertise in sociocultural theory and research methodology. I thank her for encouraging me to be involved in the academic environment by attending and presenting papers at conferences as a venue for broadening my knowledge. I have learned from both of them not only in the field of the body of knowledge as employed in the thesis but also as role models in supervising, motivating and encouraging my independence.

I also would like to thank the American volunteer teacher, Sarah Amit, who gave me an opportunity to observe her class and all the Vietnamese students who volunteered to participate in the research. I am thankful to the senior student who had the courage to volunteer as a special research participant. All the enthusiasm of the participant students made it possible for me to conduct my research in a high spirit throughout my time for data collection in Vietnam. 
I am especially grateful to New Zealand Ministry of Foreign Affairs and Trade who granted me an NZAID doctoral scholarship for me to be able to come to New Zealand to do my study. I am thankful for their financial and spiritual support through the NZAID advisers of the International Student Office, Rachel Crump and Jay Neill. The research committee of the Faculty of Humanities and Social Sciences gave me several research grants to enable me to attend and present at international conferences. They lent the research equipment necessary for me to collect data in Vietnam. I thank them for their support. I also would like to express my thanks to the board of directors of my university in Vietnam for granting me study leave. I thank Mary Redmayne for helping me with the thesis editing.

To my husband, Quang, and our daughter, Nhi, I owe a special word of thanks for their ongoing support and love. My husband has been a most patient and supportive partner who in many ways put his own ambitions aside so that I could accomplish mine. My daughter's hugs and smiles have kept me highly motivated during the journey of doing research. I thank my late father for teaching me the first English words and inspiring me with the love of English learning. My beloved mother and parents-in-law, though being far away in Vietnam, have always encouraged me to pursue my academic dreams. I thank them for their love and kindness. 


\section{CONTENTS}

Abstract 1

Acknowledgments 3

Contents $\quad 5$

List of tables 9

$\begin{array}{ll}\text { List of figures } & 10\end{array}$

1. Introduction 11

$\begin{array}{ll}1.1 \text { Background } & 11\end{array}$

1.2 Statement of purpose and research questions $\quad 12$

1.3 Significance of the study 16

1.4 English teaching and learning in the Vietnamese context 18

$\begin{array}{ll}\text { 1.4.1 Social context } & 18\end{array}$

$\begin{array}{ll}1.4 .2 \text { Vietnamese cultural values } & 19\end{array}$

$\begin{array}{ll}1.4 .3 \text { Institutional context } & 21\end{array}$

1.4.4 A brief comparison of Vietnamese with English 23

1.5 Definitions of terms used in the current study 24

1.6 Structure of the thesis $\quad 26$

2. Construction of mind: Vygotsky and other theorists 28

2.1 Introduction 28

2.2 A brief biography of Vygotsky $\quad 28$

2.3 Vygotsky and colleagues $\quad 29$

2.4 Origin of sociocultural theory $\quad 29$

2.5 Vygotskian pedagogy 34

2.6 Vygotsky and contemporary theorists on the construction of mind 36

$\begin{array}{ll}2.7 \text { Behaviourism versus constructivism } & 37\end{array}$

2.8 Vygotsky and Piaget: Different views of constructivism 39

2.9 Criticisms of Vygotskian theory $\quad 40$

2.10 Activity theory 43

2.11 Vygotsky's views of learning a foreign language 45

2.12 Summary 47

3. Sociocultural theory: Interpretations and applications 48

$\begin{array}{ll}3.1 \text { Introduction } & 48\end{array}$

3.2 Interpretations $\quad 48$

3.3 Mediation $\quad 49$

3.3.1 Mediation through oral language $\quad 51$

3.3.2 Mediation through non-verbal language 53

3.4 Mediation in the Zone of Proximal Development 54

3.5 Peers and more knowledgeable peers $\quad 59$

$\begin{array}{ll}3.6 \text { Interpretations of learning } & 60\end{array}$

$\begin{array}{ll}3.7 \text { Culture } & 62\end{array}$

3.8 Tools and artifacts $\quad 63$

3.9 Affective factors $\quad 66$

$\begin{array}{ll}3.10 \text { Cultural resources } & 68\end{array}$

3.11 Applications of sociocultural theory in classroom studies $\quad 70$

$\begin{array}{ll}3.12 \text { Sociocultural theory and EFL approaches } & 77\end{array}$

3.13 Sociocultural theory as a conceptual framework for the $\quad 78$ current study

$\begin{array}{ll}3.14 \text { Summary } & 80\end{array}$

4. Research methodology $\quad 81$ 
4.1 Introduction $\quad 81$

4.2 Research design $\quad 81$

4.3 Qualitative approach $\quad 84$

4.4 Ethical considerations $\quad 85$

4.5 Fieldwork 88

4.6 Pilot study $\quad 88$

4.7 The class unit of work $\quad 90$

4.8 Participants in the main study $\quad 92$

4.8.1 The students $\quad 92$

4.8.2 The senior student $\quad 93$

4.8.3 The classroom teacher $\quad 94$

4.8.4 The roles of the researcher 95

4.9 Classroom physical set-up $\quad 97$

4.10 Data collection $\quad 99$

4.10.1 Audio recordings $\quad 99$

4.10.2 Video recordings 99

$\begin{array}{ll}4.10 .3 \text { Journals } & 100\end{array}$

4.10.4 Interviews 101

4.11 Identification 103

4.12 Analysis 103

4.12.1 Analysis of the audio transcriptions of group work 104

4.12.2 Videotape analysis 107

4.12.3 Journal analysis 107

4.12.4 Interview analysis 108

$\begin{array}{ll}\text { 4.12.5 Artifact analysis } & 109\end{array}$

$\begin{array}{ll}4.13 \text { Research validity } & 109\end{array}$

4.14 Research assumptions $\quad 111$

$\begin{array}{ll}4.15 \text { Limitations } & 112\end{array}$

4.16 Summary 113

$\begin{array}{ll}\text { 5. Group discussion of topics } & 114\end{array}$

$\begin{array}{ll}5.1 \text { Introduction } & 114\end{array}$

5.2 Topics 114

$\begin{array}{ll}5.3 \text { Journals } & 120\end{array}$

5.3.1 Unassisted group discussions $\quad 120$

5.3.2 Assisted group discussions 121

5.3.3 Knowledge of the topics $\quad 122$

5.3.4 Students' evaluation of the topics $\quad 125$

$\begin{array}{ll}5.4 \text { Interviews } & 127\end{array}$

5.4.1 Unassisted peer groups $\quad 127$

5.4.2 Assisted peer groups 128

5.5 Group work with peers or with peers and the more $\quad 130$

knowledgeable peer

5.6 Video recordings $\quad 131$

5.6.1 Starting snapshots $\quad 132$

5.6.2 Ending snapshots $\quad 133$

5.6.3 Gestures 134

5.7 Discussion and conclusion 136

6. Opportunities to speak 138

$\begin{array}{ll}6.1 \text { Introduction } & 138\end{array}$

6.2 English, Vietnamese and combined utterances 139 
6.3 On and Off-the-assigned-topic utterances $\quad 142$

$\begin{array}{ll}6.4 \text { Utterance allocation } & 145\end{array}$

6.4.1 Utterance allocation at the beginning of a discussion $\quad 145$

6.4.2 Utterance allocation in the middle of a discussion $\quad 147$

6.4.3 Utterance allocation at the end of a discussion 149

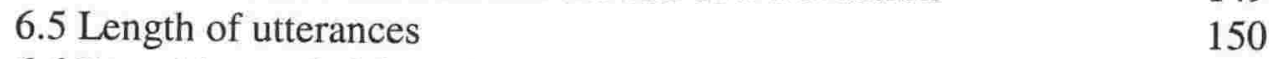

6.6 Repetition and elaboration 151

$\begin{array}{ll}6.7 \text { Journals } & 154\end{array}$

6.8 Interviews 156

$\begin{array}{ll}\text { 6.9 Discussion and conclusion } & 157\end{array}$

$\begin{array}{ll}\text { 7. Peer mediation through oral language } & 159\end{array}$

$\begin{array}{ll}7.1 \text { Introduction } & 159\end{array}$

$\begin{array}{ll}7.2 \text { Negotiation in the group work } & 159\end{array}$

$\begin{array}{ll}\text { 7.2.1 Negotiation of task planning and procedures } & 160\end{array}$

$\begin{array}{ll}\text { 7.2.2 Negotiation of understanding } & 168\end{array}$

7.2.3 Mediation through the first language $\quad 176$

$\begin{array}{ll}7.3 \text { Unsuccessful negotiation } & 177\end{array}$

$\begin{array}{ll}7.4 \text { Discussion and conclusion } & 180\end{array}$

$\begin{array}{ll}8 . \text { Learning vocabulary } & 181\end{array}$

8.1 Introduction 181

8.2 Audio transcriptions $\quad 183$

8.3 Unsuccessful negotiations of word meanings and forms $\quad 189$

8.4 The first language and learning English vocabulary 190

$\begin{array}{ll}8.5 \text { Journals } & 191\end{array}$

$\begin{array}{ll}\text { 8.5.1 New words } & 191\end{array}$

$\begin{array}{ll}\text { 8.5.2 Joint-learning of vocabulary } & 192\end{array}$

8.5.3 Collective memory in learning vocabulary 193

$\begin{array}{ll}\text { 8.5.4 Report of unsuccessful learning vocabulary } & 194\end{array}$

$\begin{array}{ll}8.6 \text { Interviews } & 195\end{array}$

$\begin{array}{ll}\text { 8.7 Discussion and conclusion } & 196\end{array}$

$\begin{array}{lr}\text { 9. Artifact use } & 198\end{array}$

$\begin{array}{ll}9.1 \text { Introduction } & 198\end{array}$

9.2 Audio transcriptions $\quad 199$

$\begin{array}{ll}\text { 9.2.1 The textbook } & 199\end{array}$

$\begin{array}{ll}\text { 9.2.2 Other classroom artifacts } & 204\end{array}$

$\begin{array}{ll}9.3 \text { Video recordings } & 206\end{array}$

9.4 Artifact use in other classes $\quad 207$

9.5 Vietnamese culture as mental artifacts $\quad 208$

$\begin{array}{ll}\text { 9.6 Discussion and conclusion } & 210\end{array}$

10. Students' feelings $\quad 213$

$\begin{array}{ll}10.1 \text { Introduction } & 213\end{array}$

$\begin{array}{ll}10.2 \text { Laughter } & 213\end{array}$

10.2.1 Laughing at the ideas related to the tasks 214

10.2.2 Students' laughing at each other 215

10.2.3 Laughing at Vietnamese cultural beliefs and practices $\quad 215$

10.2.4 Laughing because of the senior student's humorous remarks 216

10.2.5 Off-task laughter $\quad 216$

$\begin{array}{ll}10.3 \text { Enjoyment } & 217\end{array}$

10.4 Group members' enjoyment $\quad 220$

$\begin{array}{ll}10.5 \text { Stress } & 222\end{array}$ 
10.6 Emotions and learning $\quad 224$

$\begin{array}{ll}10.7 \text { Other feelings } & 226\end{array}$

10.8 Discussion and conclusion $\quad 228$

11. English outside the classroom 230

11.1 Introduction $\quad 230$

11.2 Motives to learn English $\quad 230$

11.2.1 Choosing English as a major $\quad 231$

11.3 Learning English outside the classroom 232

$\begin{array}{ll}11.3 .1 \text { Vocabulary } & 232\end{array}$

11.3.2 Reading $\quad 233$

11.3.3 Resources $\quad 235$

11.4 Using English outside the classroom 236

11.4.1 Using English with foreigners $\quad 236$

11.4.2 Using English with Vietnamese friends $\quad 237$

11.4.3 Speaking English at home 238

11.5 Summary 238

12. Discussion of overall findings: Theoretical contributions and practical 240

implications

12.1 Introduction

240

12.2 Similarities $\quad 241$

12.3 Differences $\quad 243$

12.4 The roles of peers in different group settings 246

12.5 The first language use $\quad 250$

12.6 Social interaction for task management 252

12.7 The roles of artifacts in the classroom 253

12.8 Mediation as a complex process 256

12.9 Emotions in participating in group work $\quad 257$

$\begin{array}{ll}12.10 \text { Vietnamese culture in the English classroom } & 259\end{array}$

12.11 Group work as viewed in EFL pedagogy 264

12.12 Changes in EFL in Vietnam 266

12.13 Implications $\quad 268$

12.14 Areas for further research $\quad 272$

$\begin{array}{ll}12.15 \text { Conclusions } & 274\end{array}$

$\begin{array}{ll}\text { References } & 277\end{array}$

Appendix A: Ethics application letter to an institution in Vietnam 291

Appendix B: Information sheet for the teacher(s) 293

Appendix C: Consent to participation in research: Teacher 295

Appendix D: Information sheet for students 296

Appendix E: Consent to participating in research: Students 298

Appendix F: Journal questions for unassisted students 299

Appendix G: Journal questions for assisted students 301

Appendix I: Interview questions $\quad 303$

Appendix J: Summary of categories and coding 305

Appendix K: A sample of audio transcription 307

Appendix L: A sample interview transcription with a participant student 313 


\section{LIST OF TABLES}

Table 1: Number and types of gestures used by unassisted and assisted students during group discussions

Table 2: All utterances of students in unassisted and assisted peer groups 140

Table 3: On- and off-the-assigned-topic utterances

Table 4: Quality of utterances of students in unassisted and assisted peer groups

Table 5: English learning resources outside the classroom 


\section{LIST OF FIGURES}

Figure 1: Research diagram $\quad 82$

Figure 2: Diagram of the classroom setting and data collection 98

Figure 3: Utterance allocation of an unassisted peer group-Topic: Anger 145

Figure 4: Utterance allocation of an assisted peer group-Topic: Anger 146

Figure 5: Utterance allocation of an unassisted peer group-Topic: Work 147

Figure 6: Utterance allocation of an assisted peer group-Topic: Work 148

Figure 7: Utterance allocation of an unassisted peer group-Topic: 149

Taking risks

Figure 8: Utterance allocation of an assisted peer group-Topic:

Taking risks 


\section{Chapter 1}

\section{INTRODUCTION}

\subsection{Background}

After completing my bachelor's degree majoring in English in 1994, I was appointed to the position of an English lecturer at a university in Vietnam. I then went to the United States to do a master's degree in TESOL (Teaching English to Speakers of Other Languages) and returned to Vietnam to continue my teaching career. In my time working as an English lecturer, I have perceived Vietnamese students' eagerness and thirst for learning English. They usually have a good command of written English and grammar but lack oral competence and confidence in using the language. I searched for a learning theory that embraces the importance of social interaction in learning a language to help me with my teaching of English. As my search continued, I became interested in sociocultural theory developed by L. S. Vygotsky (1896-1934) and his collaborators in Russia in the 1920s and 1930s.

Sociocultural theory holds that specifically human forms of mental activity arise in the interactions we enter into with other members of our culture and with the specific experiences we have with the artifacts produced by our ancestors and by our contemporaries. (Lantolf, 2000a, p. 79)

Vietnamese culture is collectivistic. It encourages knowledge exchange and acknowledges the roles of teachers and friends in learning (more details are presented in section 1.4 of this chapter). Sociocultural theory, therefore, is compatible with Vietnamese culture in the ways it emphasises collective learning. 
All the premises of sociocultural theory, such as social interaction and shared understanding in the context of joint action in a cultural context, and the similarities to Vietnamese cultural values, offer a relevant conceptual framework for a study of group interaction in learning English as a Foreign Language (EFL) in Vietnam.

\subsection{Statement of Purpose and Research Questions}

Vygotsky (1978) referred to peers as agents for learning. He argued that we learn from others who are more knowledgeable than ourselves, either peers or adults. The basic meaning of "peer" is an "equal". We can be equal in any number of ways but in sociocultural studies of classrooms, equality has generally been interpreted as being in the same class at school. A result of such studies has been to show that when working together, more knowledgeable peers build knowledge in the less knowledgeable. However, this leaves open the question of how much more knowledgeable a peer should be in order to bring about learning in others, and whether or not there are variations in outcomes and processes according to the "distance" in knowledge between more and less knowledgeable peers. In addition, Vygotsky did not specify the size or composition of the peer group which gives the best result. Nor did NeoVygotskians. The current study explores these issues by observing two kinds of groups, who are peers in the sense that they are all students learning English at the same educational institution. One kind of group consists of five peers from the same class. This kind of group has been called an "unassisted peer group" for the purposes of this study. The other kind consists of four peers from the same class and a senior student from a more advanced class. This kind of group has been called an "assisted peer group". The establishment of two groups of the same size meant that they could be 
observed while they were engaged in the same unit of work in the classroom where other students were also working in groups. Both groups were practising speaking English and both were following the same textbook, Well Spoken (Ramsey \& ReesParnal, 1989).

Sociocultural theory was used as the conceptual framework to analyse the learning processes in the two kinds of groups. It was used in the research design and in data analysis and interpretation. Within this framework, the study explored the roles of peers in the different group settings and mediation through psychological and material artifacts. It also focused on students' feelings during group work. In addition, the study examined support for learning English outside the classroom. A secondary purpose of this study was to uncover some pedagogical issues in relation to EFL in group work in a language class. It was not an intervention study in the usual sense. I did not establish the knowledge status of the students before the group sessions or test their competence in spoken English but I introduced a senior student into twelve group sessions which normally consisted of only students from the same class.

\section{Research Questions}

Before presenting the research questions, it should be made clear that there are two distinct uses of "learning". In common usage it refers to activities with the object of learning. In socio-cultural theory learning takes on the meaning of actual gains in knowledge, facility and practice. There is however an inherent contradiction between learning as a process and learning as a gain. Students in classrooms are learning in the first sense even if they are not learning in the second. In 
the current study, students were taking part in an activity which was officially described as an opportunity to use what had been learnt. The current study focused, therefore on the learning processes and only made claims for specific gains in learning when there was firm evidence that this had occurred.

The research question is: What are the processes of using English among Vietnamese students working in unassisted and assisted peer groups? The two kinds of groups are compared, focusing on the following sub-questions:

1. What are the differences in the way a topic is discussed?

2. What are the opportunities for speaking English in the two kinds of groups?

3. How do English and Vietnamese languages, if used, play a mediational role in the discussions?

4. How is vocabulary learnt when the students are practising oral English?

5. What artifacts function as mediational tools?

6. Do students have different feelings according to the kind of group they are in?

7. What support for the learning of English is provided by the Vietnamese environment?

The first sub-question aimed to provide a background for what the students were required to do. It examined the discussion processes and whether the students gained knowledge of the topics under discussion.

A student produces an utterance in the classroom to respond in certain ways, such as to reject, question, extend, and incorporate another's utterance, or to construct an idea in collaboration with others (Wertsch \& Toma, 1995). The second sub-question focused 
on the utterances the students produced in the process of participation in group work. It examined how students contributed ideas during discussion.

Central to sociocultural theory is the concept of mediation through language. "Language is therefore not just a means by which individuals can formulate ideas and communicate them, it is also a means for people to think and learn together" (Mercer, 1995, p. 4). The third sub-question investigated how talk and interaction among group members created conditions for joint-learning and shared understanding of the topics under discussion. This research sub-question explored the roles of peers in different group settings.

"The word in language is half someone else's" (Bakhtin, 1981, p. 293). Vocabulary is a major unit of EFL through which meanings are perceived and conveyed. Therefore, the fourth sub-question with a focus on vocabulary combined both the sociocultural theory framework and the practices of pedagogy in foreign language learning.

Language can be oral or written. Although sociocultural theory has focused on oral language and the students were all required to use English, the presence of the textbook meant that written language could not be overlooked. The concept of mediation in sociocultural theory also refers to the roles of physical artifacts and their impact on the process of idea formation and learning. Artifacts encapsulate sociocultural values and knowledge. The fifth sub-question aimed to examine artifacts available to the students, such as the textbook, the teacher's instructions on the blackboard, handouts in the classroom, and other forms of learning materials. 
Vygotsky (1962, p. 8) resisted the separation of cognition and affect, emphasising instead "the existence of a dynamic system of meaning in which the affective and the intellectual unite". Working in groups can arouse feelings within the participants. Subquestion six focused on the enjoyment, stress and other feelings involved as part of participation in group discussion.

Sociocultural theory also includes informal learning outside the classroom. Subquestion seven explored the broader context of the Vietnamese environment for students' learning of English.

\subsection{Significance of the Study}

The current study has both theoretical and practical significance. It has the potential to contribute to the body of knowledge of sociocultural theory and may be of interest to sociocultural theorists. The study discusses metatheory as expressed in Vygotsky's theory of construction of mind, including psychological and material tools of which he saw speech as the principal means of acquiring knowledge and speech as the way that knowledge becomes internalised. In addition to examining the roles of peers, it explores aspects of sociocultural theory which have received relatively little attention in sociocultural classroom studies. These include the use of the textbook as a material artifact or tool, students' feelings, their cognitive activity, and cultural influences in and beyond the classroom.

Application of the metatheory to classroom practice has resulted in an "official sociocultural narrative, or at least neo-Vygotskian versions of it" (Cobb, 1998, p. 188). 
With a focus on the processes of the two kinds of groups, the findings of the current study allowed a pedagogical judgement to be made about the value of each kind of group in an EFL classroom. Practically, the findings of the current research have implications for the application of sociocultural theory to the classroom; for example, how to create effective social interaction in group work, how to develop the roles of peers and how to make the most of a textbook. In other words, the research provides empirical data about group work processes which may help classroom teachers and textbook writers. The research may inspire classroom teachers to apply sociocultural theory in their teaching practices. It should be of interest to teachers of English in Vietnam as well as TESOL and TEFL (Teaching English as a Foreign Language) lecturers, particularly where the context is similar to that of Vietnam.

In Vietnam, so far only a small number of doctoral theses (Do, 1996; Duong, 2003; Nguyen, 2003) have been written on learning and teaching English but none has studied group interactions in the classrooms. While sociocultural theory has been embraced by many theorists and classroom teachers in other countries, there has been little research with a sociocultural theory framework in Vietnam. Sullivan $(1996,2000)$ appears to have been the first, investigating language playfulness in the Vietnamese classroom using a sociocultural framework. Tran (2003) discussed storybook reading among the first graders in Vietnam in a Vygotskian developmental framework. The current research seems to be the third example of the use of sociocultural theory to investigate the processes of learning English in Vietnam. 
In Vietnam, English teaching is prescribed in a curriculum written by the Ministry of Education and Training (MOET). It is useful to approach a natural classroom to investigate the learning processes that are the result of this curriculum.

\subsection{English Teaching and Learning in the Vietnamese Context}

\subsubsection{Social Context}

In December 1986, a policy of educational, economic and social openness known as doi moi was instituted in Vietnam. An outgrowth of this policy has been an increase in social and educational exchange, which has had the result of a growing demand for English (Gayle, 1994). The role of English as the international language for business is being promoted in the nation's rush to join the international marketplace and as the need for communication with other countries where English is used as a second language or foreign language increases (Mydans, 1995). Additionally, the need for English is being promoted to assist the integration of Vietnam into the world. "English in Vietnam is extremely important in Vietnam's promotion of international development programmes and its participation in international organisations" (McKay, 1999, p.1).

Since a good command of English promises future professional advancement, all Vietnam has been described as having an "English fever" (Denham, 1997). Vietnamese generally learn English not in order to live and work in an English speaking country but to have a good command of written and spoken English. They also learn English to pass compulsory English exams for high school graduation, to be able to take the 
university entrance exams, to function well in their jobs in foreign companies, or to obtain qualifications for such jobs.

\subsubsection{Vietnamese Cultural Values}

Different ideologies have shaped Vietnamese cultural values. First, Vietnam, through being a colony of China for thousands of years, has been influenced by the Chinese ideology of Confucianism which holds the teacher in high esteem (Ellis, 1995; Kramsch \& Sullivan, 1996). This reverence for teachers in Vietnam still has its effect in classrooms. In addition, the Confucian tradition of education encourages little selfreflection, divorces academic study from reality and aims at achieving social stability through observance of a rigid system of hierarchy (McCook, 1998). The result of this tradition is evident in the current pre-eminence of teacher-centredness and grammarcentredness in classrooms, while the students are seen as passive recipients. Nguyen (as cited in Kramsch \& Sullivan, 1996), however, pointed out that the Confucian notion of mutual obligation and membership in a group encourages the students to share knowledge with each other. The Confucian value of co-operation provides a favourable condition for group work (Flowerdew, 1998).

It is perhaps these values that have led to students in Vietnam having a strong relationship with their classmates, "The assumption of life-long relationships and obligations brings with it an expectation of cooperation and coordination in class, as well as hierarchy in relationships" (Sullivan, 1996, p. 34). Park (2000) pointed out that compared with Anglo or East Asian students, Southeast Asian students in general and Vietnamese students in particular seem to have a higher preference for group learning. 
This preference for group learning may be attributable to cultural characteristics that value cooperation and mutual assistance among the clans and villagers. This collectivist culture stresses the importance of the group over individual needs (Gudykunst, 1998; Hofstede, 1997). Additionally, in a collectivist culture, interpersonal conflict and confrontations that disrupt group harmony are to be avoided (Silka \& Tip, 1994).

Second, the major religion in Vietnam is Buddhism. Buddhist values tend to support the avoidance of disharmony, strengthen close ties in human relationships and encourage parents to become involved in their children's education (Pham, 2000). "Buddhist teaching advocates an adherence to the 'middle way' or moderation, which may have deterred any moves to take on 'radical' new approaches to language teaching, a feature that appears to be inherent in Western education" (McCook, 1998, p. 12).

With regard to learning, Vietnamese people are known to be hard working and studious. Vietnamese learners expect to learn from their teachers and from their peers. In Vietnam, teachers are highly respected, "Nhất tụ vi su, bán tụ vi su" "One who teaches you a word or even half a word is your teacher". In addition, students usually consider knowledge resides in their teachers, "Không thầy đố mầy làm nên" "Without a teacher, you cannot complete your work successfully". These proverbs show respect for those who are more knowledgeable and the importance of obtaining knowledge as part of the Vietnamese tradition. Knowledge in the Vietnamese context also implies that one has to cooperate with others to share wisdom and broaden knowledge, "Một cây làm chẳng nên non, ba cây chụm lại nên hòn núi cao" "A tree cannot make a mountain, three trees can make a high one". In certain situations, friends are considered 
to be more helpful than teachers, "Học thầy không tày học bạn" "It is easier to learn from friends than from teachers".

Generally, knowledge in the Vietnamese context implies both internal effort by the learners and external factors from teachers, friends or whoever is providing knowledge.

\subsubsection{Institutional Context}

In Vietnam, the MOET is responsible for such tasks as developing educational policies and the standardisation of curricula and testing. Most EFL institutions are public or semi-public and are thus subject to Ministry guidelines. English is taken as a compulsory subject by secondary and university students, and high school entrance and graduation examinations include English. Do (1999, p. 30) provides the background of learning English in Vietnam:

University students are now required to take foreign languages for their graduation exams. In addition, many universities require students of all majors to take a foreign language at the entrance exams. Post-graduate education and the granting of faculty positions also require foreign language proficiency. Foreign languages, especially English, are increasingly used widely in education and daily-life activities. As a matter of fact, the status of foreign languages, particularly English, has recently been reconfirmed by an Order, signed by the Prime Minister (August 15, 1994), in which government officials are required to study foreign languages, usually English. It should be noted here that, in contemporary Vietnam, there has never been a stronger, clearer decision concerning foreign language education policy and planning made at the highestlevel authority.

With regard to foreign language learning, a variety of foreign languages are taught in language-training institutions including Chinese, English, French, German, Japanese, and Russian; however, about $80-85 \%$ of all foreign language students study English (Gayle, 1994). As for English learning and teaching materials, there is a dearth of 
teaching resources for Vietnamese learners of English. The main teaching resource is the teacher (Denham, 1992; Lewis, 1996). Most textbooks are published in Englishspeaking countries and are used in Vietnam without being adapted to fit in with the new learning environment. Classrooms are generally furnished with fixed benches arranged in parallel rows facing a chalkboard. Classes at university level gather either in the morning or in the afternoon and study in the same room for the whole semester. Class size for students at university who major in English is typically 40-50 students, but this varies from university to university and according to class level. Within the context of large classes and varied levels of English among the students, student participation has been referred to as a problem (Le, 2002a).

Many methods of teaching English can be found in Vietnamese schools and their use varies depending on the levels of instruction and purposes. Eyring and Silverman (1996) found that Vietnamese teachers of English employed methods, such as an Audio-Lingual approach (teaching language with drills), the Grammar-Translation approach (focusing on grammar, translating from mother tongue to target language or vice versa), the Direct Method (with all learning in the target language), and the Communicative Approach (focusing on meaning and fluency). However, among these, the Grammar-Translation method, which is closely connected to traditional Vietnamese education, is still dominant. In foreign language classes, translation accompanied by an explanation and practice for certain grammatical points takes most of the class time. Lewis (1996), when conducting a workshop on teaching English in Vietnam, found that Vietnamese teacher trainees had few chances to use English outside the classroom. The class is mainly monolingual without genuine communicative reasons to speak English to one another. Lewis pointed out that, as there is a high level of importance placed on 
using English in Vietnam, "students need to see the point of talking as a way of becoming fluent in language" (p. 9). She also suggested that students should be shown how to be better language learners and reminded of why they are learning English.

In general, there is a discrepancy between the need and the reality of learning and teaching English in Vietnam. Although the students have high objectives and goals to learn English, they face a shortage of resources, the opportunity to use the language, and exposure to a good English speaking environment.

\subsubsection{A Brief Comparison of Vietnamese with English}

Vietnamese is monosyllabic. It possesses six tones which are indicated in the Romanised script by five diacritical marks, the absence of a diacritic indicating the sixth. They are high rising as in bá, high level as in $b a$, low falling as in bà, fall rise in $b a ̉$, high creaky as in $b \tilde{a}$, and low creaky in $b a$. The earliest writing comprised ideograms, either Chinese characters or others developed from these. In Vietnam

during the $17^{\text {th }}$ century, European missionaires and priests created a new Romanised script to help them with translation and publication of their religious materials and to replace the character writing. Alexandre de Rhodes among many other missionaries and anonymous Vietnamese developed the first system of modern Vietnamese orthography (Nguyen, 1994). Modern Vietnamese differentiates words by tone and makes great use of syntax and particles for grammatical purposes. However, in sentence structure, the Vietnamese word order in general is similar to that of English with subject-verb-object (S-V-O), which is a facilitating factor helping Vietnamese learners of English to understand word order. 
Regarding orthography, Vietnamese is entirely phonetic in spelling. An example of Vietnamese orthography is, "Chúng tôi học tiếng Anh ở trường" "We learn English at school".

\subsection{Definitions of Terms Used in the Current Study}

The following key terms are used in this thesis:

\section{Sociocultural discourse analysis}

Sociocultural discourse analysis differs from "linguistic" discourse analysis in being less concerned with the organisational structure of spoken language, and more with its content, function and the ways shared understanding is developed, in social context, over time. (Mercer, 2000, p. 4)

A socio-cultural analysis looks for evidence of processes. The strength of socio-cultural discourse analysis, according to Mercer, is its sensitivity to culture, context and the ways talk enables educational activity to progress, minute by minute. The analysis of classroom discourse provides one possible means through which educators across disciplines can explore how students collectively and individually construct disciplinary knowledge. Questions that orient the discourse analysis of the current data are: (1) How is language used to create joint knowledge and understanding? and (2) How do students help other students to learn? (Adapted from Mercer, 1995, p. 84).

Higher mental functions, according to Vygotsky (1986), include voluntary attention, memory, intentional memory, planning, interpretive strategies, creative imagination, verbal thinking, and forms of logic and rationality which develop on the foundation of lower mental processes (direct perception, eidetic memory, involuntary attention, and preverbal thinking) through mediation by psychological tools or signs. Lower mental 
processes are subject to the control of the environment, whereas higher mental processes are subject to self-regulation (Manning \& Payne, 1993).

Artifacts include both physical objects and immaterial cultural tools (Wartofsky, 1979; Wertsch, 1998). They are the signs and sign systems which Vygotsky (1981, p. 137) defined as, "language; various systems for counting; mnemonic techniques; algebraic symbol systems; works of art; writing; schemes, diagrams, maps, and mechanical drawings; [and] all sorts of conventional signs". Wertsch (1998) stated that physical objects are artifacts in the sense of historical artifacts that continue to exist after the humans who used them have disappeared. According to Wertsch, spoken language is an immaterial cultural tool. Artifacts are the material and conceptual tools and practices that the students used in the goal-oriented group discussion. They include for example a textbook, a blackboard, and the Vietnamese cultural practices and representations.

Unassisted peer group: A group of five students from the same level who work together in English speaking activities.

Assisted peer group: A group of four students from the same level who work with the assistance of the senior student from a more advanced class.

Utterances: An utterance is a unit of speech communication. It defines who speaks and the boundaries between speakers, which are determined by a change of speaking subjects. It also indicates the flow of the interaction (Bakhtin, 1986). Bakhtin emphasised the speaker's response-orientation and considered that each utterance is filled with echoes and reverberations of other utterances. 
Speech can exist in reality only in the form of concrete utterances of individual speaking people, speech subjects. Speech is always cast in the form of an utterance belonging to a particular speaking subject, and outside this form it cannot exist. (Bakhtin, 1986, p. 71)

EFL (English as a Foreign Language): refers to learners whose first language is not English and who are learning English in their home countries, for example, Vietnamese and Chinese learners of English in their home countries.

ESL (English as a second language): refers to learners whose first language is not English but who are learning English in an English speaking country.

TESOL: Teaching English to Speakers of Other Languages.

University students: The study focuses on Vietnamese students who study four years at university to obtain their bachelor's degree in English. Their ages are between 18 and 22.

\subsection{Structure of the Thesis}

This thesis consists of 12 chapters. Chapter 1 has introduced the research, its purposes and research questions. It also summarised aspects of English learning and teaching in Vietnam. The literature review is presented in two chapters: 2 and 3. Chapter 2 discusses the formation of sociocultural theory taking into consideration its origin. It considers contemporary theorists of Vygotsky from the point of view of epistemology and the construction of mind. Chapter 3 continues with details of sociocultural theory and of how it has been interpreted and applied in classroom research. Chapter 4 
presents the research methodology. The results are reported in Chapters 5-11 in accordance with the research questions. The last chapter provides a summary of the thesis, drawing out the similarities and differences of group processes in the two kinds of group settings. It then synthesises all the findings according to emerging sociocultural themes and with relevance to EFL approaches. The chapter also discusses theoretical contributions and practical recommendations as well as further areas of research.

In the literature review and the result chapters of my thesis, where appropriate, I have ordered the presentation according to metatheory to provide a general orientation with ontological and epistemological assumptions. Generally, I start with the level of metatheory in Vygotsky's theory of construction of mind and then provide data to discuss its application.

Chapter two will start the metatheory discussion with Vygotsky's philosophical ideas of construction of mind and the origin of sociocultural theory. 


\section{Chapter 2 \\ CONSTRUCTION OF MIND: VYGOTSKY AND OTHER THEORISTS}

\subsection{Chapter Introduction}

This chapter introduces Vygotsky's life and works and compares his philosophy and pedagogy with those of other theorists. The chapter also discusses the origin of sociocultural theory.

\subsection{A Brief Biography of Vygotsky}

Lev Semyonovitch Vygotsky was born on November 5, 1896 in Orsha in Russia. During his life, he participated in different academic fields, such as completing a law degree then teaching literature and psychology, and writing a $\mathrm{PhD}$ thesis on The Psychology of Art (1917-1923). From 1925-1934, he conducted research in psychology, "defectology" and "mental abnormality" (Luria, as cited in Cole, JohnSteiner, Scribner \& Souberman, 1978, p. 15). According to Luria (1979), Vygotsky was a genius with a breadth of knowledge in many fields, drawing heavily on German, French, English, and American writings. He and his colleagues read widely in the psychology of their day, translating and writing prefaces to the works of Dewey, James, Janet, Piaget, Freud, Levy-Bruhl, Durkheim, the Gestalt psychologists and more (Cole, 1996, p. 36).

Vygotsky was influenced by different scholars, especially A. A. Potebriya and Alexander von Humboldt, who were interested in the effect of language on thought 
processes. He was particularly interested in work of V. A. Wagner on the comparative study of animal behaviour. Vygotsky died of tuberculosis on June 11, 1934, aged 38. For detailed descriptions of Vygotsky's life and works, see Cole et al. (1978) and Blanck (1990).

\subsection{Vygotsky and Colleagues}

Before his death, Vygotsky, his colleagues, mainly Leontiev, Luria, and their students established a laboratory in Kharkov headed by A. N. Leontiev. Luria and Vygotsky initiated studies, aiming at examining the cognitive consequences of rapid social change and the specific impact of schooling. Luria was among the researchers who later went on to carry out pioneering work in developmental and neuro psychology. The Kharkovites later veered away from Vygotsky's position regarding the symbolic mediation of mental life and embraced the notion that mediation arises fundamentally from practical activity with the world of objects. The Kharkovites, largely in the person of A. N. Leontiev, proposed a formal theory of activity. Section 2.10 in this chapter presents more information on activity theory.

\subsection{Origin of Sociocultural Theory}

L. S. Vygotsky developed and systematised sociocultural theory in the 1920s and 1930s with his collaborators in Russia (see John-Steiner \& Mahn, 1996; Kozulin, 1998; Moll, 1990; Wells, 1999; Wertsch, 1985). Sociocultural theory is based on the concept that human activities take place in cultural contexts and are mediated by language and other symbol systems. Human activities can be best understood when investigated in the 
context of their cultural historical development. Wertsch (1985) summarised the three themes in Vygotsky's theoretical approach: a reliance on a genetic, or developmental method; an emphasis on higher mental functioning in the individual and its origin in social life; and the idea that human mental functioning is mediated by tools and signs.

There are different cultural historical factors that mediated the process of the development of sociocultural theory. Vygotsky grew up with the Revolution in Russia in 1917. Russian society was infused with the energy of revolutionary change. Vygotsky, together with other young scholars working in the general area of psychology, was searching for a future science of psychology. He was interested in the relation between conditioned reflexes and humans' conscious behaviour (Luria, 1979). In Russia at that time, Bekhterev and Pavlov were well known for their opposition to subjective psychology, in which consciousness was a key concept. Vygotsky defended the position that consciousness had to remain in psychology, arguing rather that it must be studied by objective means. Vygotsky and his colleagues (e.g. Luria, 1979) considered that the natural science base of adaptive adjustments to the environment provided a materialistic underpinning to the study of mind.

Pavlovian reflexes might serve as the material foundation of mind, but they did not reflect the structural realities of complex behaviour or the properties of higher psychological processes. Just as the properties of water could not be discovered directly from knowing that water consists of two hydrogen and one oxygen atom, the properties of a psychological process such as voluntary attention could not be recovered directly from knowing the way in which individual cells respond to novel stimuli. (Luria, 1979, p. 41)

Vygotsky criticised Pavlov and Bekhterev because a human being is not at all "a sack of skin filled with reflexes", and the brain is not "a hotel for a series of conditioned reflexes accidentally stopping in" (Joravsky, 1987, p. 195). Conditioned reflexes could not explain higher nervous functions, such as reasoning and speaking. Reflexology 
dominated Soviet psychology until the mid-1920s, when Vygotsky and his collaborators called psychologists to the social or "historic cultural" study of the conscious mind that is appropriate to Marxists (Jarovsky, 1987, p. 198).

The interest in higher psychological functions had drawn Vygotsky to Marxist theory. According to Luria (1979), Vygotsky was a leading Marxist theoretician. One of the key concepts from Marx which he later used to develop his theoretical framework to study higher psychological functions is:

The spider carries out operations reminiscent of a weaver, and the boxes, which bees build in the sky, could disgrace the work of many architects. But even the worst architect differs from the most able bee from the very outset in that before he builds a box out of boards, he has already constructed it in his head. At the end of the work process he obtains a result, which already existed, in his mind before he began to build. The architect not only changes the form given to him by nature, within the constraints imposed by nature, but he also carries out a purpose of his own which defines the means and the character of the activity to which he must subordinate his will. (Capital, as cited in Luria, 1979, p. 42)

Influenced by Marx, Vygotsky concluded that the origins of higher forms of conscious behaviour were to be found in the individual's social relations with the external world. "But man $[\mathrm{sic}]$ is not only a product of his environment; he is also an active agent in creating that environment" (Luria, 1979, p. 43).

Luria further pointed out that Vygotsky liked to call his approach to discovering the sources of specifically human forms of psychological activity as "cultural", "historical, or "instrumental" psychology. Each approach emphasised different sources of the general mechanism by which society and social history mould the structure of those forms of activity that distinguish man from his animal neighbours.

"Instrumental" referred to the basically mediated nature of all complex psychological functions. Unlike basic reflexes, which can be characterised by a stimulus-response process, higher functions incorporate auxiliary stimuli, which 
are typically produced by the person himself. The adult not only responds to the stimuli presented by an experimenter or by his natural environment, but also actively modifies those stimuli and uses his modifications as an instrument of his behaviour. We know some of these modifications through folk customs such as tying a string around one's finger in order to remember more effectively. (Luria, 1979, p. 44)

The "historical" element, according to Luria, merged into the cultural one. The "cultural" aspect of Vygotsky's theory involved the socially structured ways in which society organises the kinds of tasks that the growing child faces and the kinds of tools, both mental and physical, that the young child is provided with to master those tasks. One of the key tools invented by mankind is language, and Vygotsky placed special emphasis on the role of language in the organisation and development of thought processes (Luria, 1979, p. 44).

Vygotsky combined the historical materialism developed by Marx and the concept of labour, tool use, and mediation by Engels in sociocultural theory. Vygotsky was first concerned with the transformation of elementary psychological processes into complex ones. The theory has expanded in a variety of ways. From very early in his career, Vygotsky clearly viewed Marxist thought as a valuable scientific resource. "A psychologically relevant application of dialectical and historical materialism would be one accurate summary of Vygotsky's sociocultural theory of higher mental processes" (Cole et al., 1978, p. 6).

Marx's theory of society considers that historical changes in society and material life produce changes in the nature of consciousness and behaviour. In focusing on human learning and development, Vygotsky took a new direction. He viewed learning and development as occurring in interaction with others in society and in the context of 
culture. "He [Vygotsky] creatively elaborated on Engels' concept of human labour and tool use as the means by which man changes nature and, in so doing, transforms himself" (Cole et al., 1978, p. 7). Thus, for Vygotsky, in the tradition of Marx and Engels, the mechanism of individual developmental change is rooted in society and culture (Cole et al., 1978).

Vygotsky (1978, p. 54) provided a synthesis of the relationship between the use of signs and tools as follows,

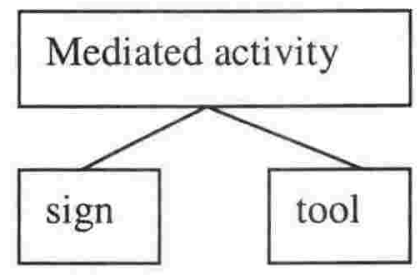

The use of both tools and signs involves mediated activity, but signs are internally oriented and a means of psychological influence to master oneself. Tools are externally oriented to master and triumph over nature. "A sign can be considered only metaphorically as a tool" (Wells, 2002, p. 49). Tools and signs are the "auxiliary means" by which interactions between subject and object are mediated (Cole \& Engeström, 1993).

In addition to mediation (for detailed discussion of mediation, see section 3.3, Chapter 3), Vygotsky especially emphasised the social factors in children's cognitive development:

Human learning presupposes a specific social nature and a process by which children grow into the intellectual life of those around them. (Vygotsky, 1978, p. 88) 
In this sense, sociocultural theory views human agency as non-individualistic and the cultural nature of human psychological tools extends human agency beyond that of a given individual. Central to sociocultural theory is the use of language. "The word is the most direct manifestation of the historical nature of human consciousness" (Vygotsky, 1978, p. 285). Vygotsky (1978) saw that external social speech was internalised through mediation. In this way, sociocultural theory links society and culture to mind through mediation. Language as a tool of the mind bridges the individual understanding of ourselves and particular contexts and situations within the world. In a society, individuals are interdependent, and for a child, language plays a key role in maintaining interdependence and developing their thinking:

The child's intellectual growth is contingent on his mastering the social means of thought, that is, language. (Vygotsky, 1962, p. 51)

In short, Vygotsky and colleagues developed sociocultural theory with an interest in the development of human intellectual and higher functions through the process of social interaction and the mediation of language and tool use. Sociocultural theory drew from historical materialism by Marx and tool use by Engels. Interpretations and applications of sociocultural theory are discussed in Chapter 3. The following section presents Vygotskian pedagogy.

\subsection{Vygotskian Pedagogy}

Vygotsky (1978) was particularly interested in the use of his ideas in the classroom. Originally, Vygotsky was interested in children being educated in their first language. The process puts more emphasis on natural learning and instruction between adults and children. According to Vygotsky, learning and development are not the same. Adults 
need to pay attention to their instructions to engage the children in the process of learning, which leads to development. Vygotsky made these propositions about the relationship between teaching and development:

The only good kind of instruction is that which marches ahead of development and leads it; it must not be aimed much at the ripe as at the ripening functions. (Vygotsky, 1962, p. 104)

What the child can do in co-operation today he can do alone tomorrow. (1962, p. 41)

Vygotsky (1978) also confirmed the idea of learning leading to development and learning happening in interaction with the environment and with others:

Learning awakens a variety of internal developmental processes that are able to operate only when the child is interacting with people in his environment and in cooperation with his peers. Once these processes are internalised, they become part of the child's independent developmental achievement. (p. 90)

The process of social instruction and learning can be informal or formal but formal instruction proves to be more crucial in Vygotsky's perspectives. He wrote,

Instruction is one of the principal sources of the school child's concepts and is also a powerful force in directing their evolution: it determines the fate of his total mental development. (Vygotsky, 1962, p. 85)

In summary, Vygotsky's pedagogy includes these factors: learners who are able to communicate and understand ideas from others, adults or peers who are able to engage learners in the process of instruction or expressing ideas, and language as a means of social interaction for both informal and formal instruction. The process of learning in Vygotsky's view is about developing the ability to engage with, and participate in, a classroom activity, or a cultural setting. 


\subsection{Vygotsky and Contemporary Theorists on the Construction of Mind}

As seen from the discussions in sections 2.4 and 2.5, Vygotsky was interested in the construction of mind. Traditional empiricism treated the mind as an empty bucket, or a blank page waiting to be filled with sense impressions or the results of reasoning. The mind was considered as a passive "mirror of nature" (Ernest, 1995). Vygotsky was against traditional empiricism; however, he was not the only one to view knowledge as socially rather than individually constructed. Social learning and interaction have interested theorists from a variety of countries. In the United States within the same period, John Dewey was working on learning by experience, which aims at fostering social interaction and contact in the process of learning. According to Dewey:

The individual is always a social individual, the self is a social product, and is always subject to change through experience, which is always in some sense social experience. The mind is not "given" but "emergent", meanings and patterns of behaviours develop through participation in group life, of which the central instrumentality is language. (Skilbeck, 1970, p. 30)

The Austrian philosopher, Ludwig Wittgenstein, also considered that knowledge was socially created:

In the later Wittgenstein, the basic reason why the theory of knowledge cannot treat knowledge as primarily the possession of individuals is this: All meanings are created in the public domain in the context of collective situations and activities. (Toulmin, 1999, p. 58, italicised as in originals)

Vygotsky's work converges on Wittgenstein's in their shared concern with practice.

"For both men, language has a definite meaning only when it is related to a given constellation of practical activities" (Toulmin, 1999, p. 59).

Holzman (1996) claimed that a striking parallelism can also be found in the writings of

G. H. Mead, an American philosopher, and Vygotsky. She pointed out, "Both 
Vygotsky and Mead make the point that social interaction is the basis of an individual's development: 'psychological processes for Vygotsky'; 'mind for Mead'”(p. 86).

The French sociologist, Durkheim (1956, p. 71) also wrote on the topic of education and social life:

Education is the influence exercised by adult generations on those that are not yet ready for social life. Its object is to arouse and to develop in the child a certain number of physical, intellectual and moral states, which are demanded of him by both the political society as a whole and the special milieu for which he is specially destined.

Vygotsky's view of construction of mind differs from other theorists in that it places a greater focus on the way social interaction in cultural contexts transforms knowledge in individuals.

\subsection{Behaviourism versus Constructivism}

In this section, Vygotsky's view of learning is viewed from an epistemological viewpoint. Epistemology is the study of knowledge, its structure and acquisition; what constitutes knowledge and how we come to know. There are different theories of learning with divergence in emphasis on whether the individual, or society, is more important. Behaviourism was an early approach to understanding learning. Behaviourism explains learning as a system of behavioural responses to physical stimuli. Pedagogy according to this theory emphasises skill and sub-skill practice, reinforcement, practice, and external motivation (Gagne, 1965; Skinner, 1953). Behaviourism views learners as passive recipients. "Learners are diagnosed in terms of deficiencies, called 'needs', then taught until 'mastery' defined as behavioural competence is achieved at each level" (Fosnot, 1996, p. 8). Behavioural theory supports 
direct transmission teaching approaches which critics have judged as bad practices (Jordan, 2003).

Unlike behaviourism, constructivism views learning as constructions of the active learner. Constructivism examines the relation between the mind and the world. Constructivism is defined as,

A theory about knowledge and learning; it describes both what "knowing" is and how one "comes to know." Based on work in psychology, philosophy, and anthropology, the theory describes knowledge as temporary, developmental, non-objective, internally constructed, and socially and culturally mediated. Learning from this perspective is viewed as a self-regulatory process of struggling with the conflict between existing personal models of the world and discrepant new insights, constructing new representations and models of reality as a human-making venture with culturally developed tools and symbols, and further negotiating such meaning through cooperative social activity, discourse, and debate. (Fosnot, 1996, p. ix)

In the theory of constructivism, learners raise their own questions, generate their own hypotheses, and formulate their own models as possibilities. Dialogue within the concept of a community of learners engenders further thinking. Ideas are accepted as truth only insofar as they make sense to the community and thus rise to the level of "taken as shared". Learners are active constructors, meaning-makers interacting with the physical and social world. There are various interpretations of constructivism. For a full description of constructivism, see Ernest (1995).

Generally, Vygotsky's theory has been described as social constructivism (Fox, 2001; Prawat, 1999; Stetsenko \& Arievitch, 1997). Social constructivism has a common set of assumptions as follows:

1. Learning and development is a process of co-construction leading to an increase in self-regulation and intentionality on the part of the individual. 
2. This process takes place within and is actively shaped by culture and historical context.

3. Language is the mediator in this process. (Prawat, 1999, p. 256)

Wink and Putney (2002) however used the terms social learning, social constructivism, and neo-Vygotskian approach interchangeably.

\subsection{Vygotsky and Piaget: Different Views of Constructivism}

Both Vygotsky and Piaget focused on construction of knowledge and cognitive thinking. However, the dimensions of interest to them were different. Nyikos and Hashimoto (1997, pp. 506-507) considered that Piaget represents the cognitive constructivist view, highlighting individual construction of knowledge in response to interaction in the physical world, but stressing the "primacy of individual cognitive development" as a "relatively solitary act" apart from social context. Vygotsky on the other hand emphasised the primacy of social interaction as the driving force and prerequisite to individuals' cognitive development through internalisation of ideas encountered in the sociocultural realm.

Piaget (1977) sought to create a biological, evolutionary view of cognitive development to explain how an individual constructs knowledge of scientific, logical, and mathematical ideas. He located the development of higher cognitive functions in the development of logical thinking, the construction of the concept of an object, and in an understanding of such constructs as space, time, and number based on an individual interaction with the physical environment. Language, according to Piaget, was only one of a number of possible systems of representation. Vygotsky on the other hand focused 
on social processes and sought to examine the representational systems that are needed to participate in such processes.

Confrey (1995, p. 202) summarised the difference between Piaget and Vygotsky's constructivism as follows:

In Piaget, the flow of construction is from one's interactions with one's nonhuman environment toward an exchange with others. In Vygotsky, the flow of conceptual development is reversed.

Both Piaget and Vygotsky have had a major influence on pedagogy. Piaget's ideas were extremely influential in mathematics education and the education of young children. Currently, it is Vygotsky's ideas that have been become popular in the context of the classroom.

In answer to the question "Where is mind?" Meacham (1996, p. 304) stated that, "Vygotsky locates mind within the interactions of individuals situated within societal, cultural, and historical contexts, whereas Piaget locates mind within the head of the individual". Thus, according to Meacham, Vygotsky and his followers pursue the question of how societal and cultural mind reproduces itself within individuals, whereas Piaget and his followers pursue the question of how individuals construct new knowledge with the potential for transformation of society and culture.

\subsection{Criticisms of Vygotskian Theory}

Despite the increasing interest in Vygotsky's ideas and works, several sociocultural theorists and social constructivists have criticised Vygotsky and his approaches to learning and development. 
Wertsch and Tulviste (1992) criticised Vygotsky for putting so much emphasis on the social and cultural processes. This view minimises the contributions made by the active individual and raises the question of how individuals are capable of introducing innovation and creativity into the system. Wertsch and Tulviste considered the ideas of some of Vygotsky's contemporaries, such as Bakhtin $(1981,1986)$ on voice and dialogicality to complement those of Vygotsky. "In Bakhtin's view, speaking always involves a concrete individual in a unique setting using language tools provided by others to create utterances" (Wertsch \& Tulviste, 1992, p. 555). These authors commented that such ideas provide concrete ways for exploring the Vygotskian account of agency as individual(s)-operating-with-mediational means. The foundation that Bakhtin focused on is the utterance as the unit of speech communication (see section 1.5, Chapter 1).

Confrey (1995, pp. 204-205) also raised several concerns over Vygotsky's model of instruction. He claimed that:

1. Seeing learning as primarily the appropriation of the adult's goals underestimates the importance of students' autonomy.

2. It has a lack of analysis of conceptual development and as a result the more knowledgeable other is unable to select more or less appropriate approaches from among the possible pedagogical routes.

3. An overtly heavy emphasis on the role of language in learning may prove less of a concern in a situation of apprenticeship, where the use of tools and the production of a product require a full engagement with the activity under examination.

4. It meant that there is a tendency to lose the epistemological freshness of child invention and its implications for the revision of expert knowledge. It leaves little room for the acknowledgement of what children teach to adults.

5. It is not clear what prevents the more experienced from using indoctrination to achieve the convergence of a child's activity to his or her own educational approaches. As a result, the Vygotskian treatment of teaching seems to have the potential to be authoritarian. 
Van der Veer and van Ijzendoorn (1985) considered socio-historical or culturalhistorical theory to be the same, claiming that Vygotsky created a dichotomy between the lower and higher processes due to an inadequate conception of the lower psychological processes (see section 1.5, Chapter 1 for definitions of higher and lower processes). They pointed out that Vygotsky considered that higher processes are the result of social interaction between children and adults. However,

Now and then, Vygotsky characterises the lower psychological processes as "natural" and the higher psychological processes as "cultural". In other words, he seems to imply that the influence of culture on the mental development of the child is brought about only by social interaction. (Van der Veer \& van Ijzendoorn, 1985, p. 5)

These authors pointed out that the child is also actively interacting with objects and is influenced by the surrounding culture.

Fox (2001) did not criticise Vygotsky in particular but social constructivism in general. Vygotsky is generally considered as representing social constructivism (see section 2.7). Fox argued that constructivism puts too great an emphasis on either teacher or those taught, which can lead to prescriptions for teaching that either ignore the learners' needs or ignore the teacher as a valuable knowledge resource. In support of the view that knowledge is constructed, rather than innate or passively absorbed, Fox claimed, "Our ability to perceive, to learn, to speak and to reason are all based on the innate capacities of the evolved human nervous system" (p. 26). He went on to criticise social constructivists for failing to account for the fact that a socially constructed reality presupposes a reality independent of all social constructions, for there has to be something for the social constructions to be constructed out of. The insistence on the intrinsically social nature of all knowledge in construction, and hence all learning, also tends towards an implausible extreme because in this case "social factors, or influences, 
alone determined all learning and all conscious thought. This would deny the individual any role or influence whatever in learning" (Fox, 2001, p. 29). Furthermore, Fox criticised the view of constructivism that learning is essentially a process of making sense because learning is also made up of practising to eliminate errors from habitual routines, and secondly because it somehow allows us to transfer our limited powers of conscious attention away from routine competence. In learning a foreign language, practising is often needed to improve language skills.

\subsection{Activity Theory}

Vygotsky (1978) introduced activity as a major explanatory concept in the study of the development of human thought, stating that "The internalisation of socially rooted and historically developed activities is the distinguishing feature of human psychology" (Vygotsky, 1978, p. 57). Later, Leontiev (1981) proposed a formal theory of activity to provide a fuller explanation of the basis of higher mental functioning than Vygotsky had initially developed. Activity theory focuses on the culturally structured interaction of people and tools to produce a mutually desired goal. The fundamental question raised by activity theory is "What is the individual or group doing in a particular setting?" (Lantolf \& Appel, 1994, p. 17). Activity theory comprises a framework on three levels: activity, action, and operation (Lantolf \& Appel, 1994). The level of activity is defined as the social, institutionally determined, setting or context based on a set of assumptions about the appropriate roles, goals, and means to be used by the participants in that setting. Examples of activity settings include play, work, education, worship and leisure time. The level of action is the level of an activity at which the process is subordinated to a concrete goal (Leontiev, 1981). The goal of an activity 
functions as a kind of regulation of the activity, and itself can be segmented into subgoals. At the operational level, physical or mental means are used to carry out the goal. The means are bound to the actual circumstances and conditions under which a goal is realised. Activity theory argues that human socio-cultural activity that gives rise to higher forms of cognition is composed of contextual, intentional, and circumstantial dimensions.

According to Daniels (2001), activity theory and sociocultural theory are related. Both attempt to provide an account of learning and development as mediated process. Both provide methodological tools for investigating the processes by which social, cultural and historical factors shape human functioning. "In sociocultural theory the emphasis is on semiotic mediation with a particular emphasis on speech. In activity theory it is activity itself which takes the centre stage in the analysis" (Daniels, 2001, p. 1).

Arievitch (2002) in his keynote address at the $5^{\text {th }}$ ISCRAT congress in Amsterdam acknowledged Gal'perin's contribution to activity theory. Gal'perin was Vygotsky's colleague and had worked with Vygotsky and Leontiev on the role of material, external activity in the formation of human mind. Arievitch pointed out that whereas Vygotsky addressed the issue of communicative aspects of joint activity on the individual's mental performance, Leontiev focused on the powerful impact of material activity on the individual's mental performance. Gal'perin considered that neither of the factors was completely sufficient.

Gal'perin's internalisation demonstrates the genetic link between external and internal activity and the particular mechanism of how external becomes internal - without a dualistic dichotomy between these two planes. As Gal'perin's work shows, it can be understood not as spatial transfer but as transformation of certain forms of an individual's activity into other forms of that same activity, 
and as a specifically human form of appropriation of new knowledge and skills. In this sense, it appears that we can overcome the dualistic dichotomy without discarding individual cognition and 'internal' plane. Instead, we can reconceptualise them in a non-mentalist way. (Arievitch, 2002, p. 9)

Activity theory has also been used in research in different fields: language and its acquisition, play, learning and instruction, therapy and addiction, and the work place (see Engeström, Miettinen, \& Punamäki, 1999).

\subsection{Vygotsky's Views of Learning a Foreign Language}

As discussed in section 2.5 of this chapter, Vygotsky focused mainly on first language learning and cognitive development. However, in some parts of his works, he discussed the issue of learning a second or foreign language. Although discussions of teaching and learning a foreign language appear infrequently in his works, in Thought and Language, Vygotsky (1962, p. 109) explained his view of the process of learning another language:

The child's strong points in a foreign language are his weak points in his native language and vice versa. In his own language, the child conjugates and declines correctly, but without realising it. He cannot tell the gender, the case, or the tense of a word he is using. In a foreign language he distinguishes between masculine and feminine gender and is conscious of grammatical forms from the beginning. Of phonetics the same is true. Faultlessly articulating his native language, the child is unconscious of the sounds he pronounces, and in learning to spell he has great difficulty in dividing a word into its constituent sounds. In a foreign language he does this easily and his writing does not lag behind his speech. It is the pronunciation, the spontaneous phonetics that he finds harder to master. Easy, spontaneous speech with a quick and sure command of grammatical structures comes to him only as the crowning achievement of long, arduous study. 
The statement above suggests a presupposition on Vygotsky's part of a necessity to teach a foreign language via "academic" methods. This is supported by Barnard (2000, p. 85) who pointed out that:

Vygotsky had very little to say about second language learners; his main reference to them (1962: chapter 5) clearly suggests that he was thinking in terms of formal instructional contexts.

Additionally, Vygotsky (1962, p. 83) had remarked that,

[Tolstoy] found that one could not teach children literary language by artificial explanation, compulsive memorising, and repetition as one does a foreign language.

Levine (1993, p. 210) also criticised Vygotsky's narrow approach to foreign language learning:

In Thought and Language (1962, 1988), he refers only to learning foreign languages under taught, disembedded conditions. The case he makes for formal learning of foreign languages bears inspection, lest it is employed uncritically to support old-fashioned non-communicative language teaching practice...Even while he quotes Tolstoy on the uselessness of teachers attempting to transfer concepts directly to their pupils through explanation, he appears to find this method acceptable when employed in foreign teaching...He then goes on to describe how in foreign language learning, as is indeed the case, one is aware of phonetic, grammatical and syntactic forms before one develops spontaneous, fluent speech. But Vygotsky then takes for granted that it is through study alone that we attain this fluency.

According to Vygotsky, "The teaching of a foreign language should be more concerned with the new language's external manifestations than with semantic aspects since meaning systems have already been constructed by learners in the acquisition of their first languages" (Levine, 1993, pp. 211- 212). Levine commented that, "Vygotsky, in using schooled foreign language learning as an example of conscious learning, left the teaching methodology uninspected" (p. 211). Levine concluded, suggesting that,

In teaching English as a foreign language, it is essential that teaching practices are framed within Vygotsky's general theory of the social basis of learning, and 
not based upon extracted comments of his that seem to support disembedded, separate development - approaches long associated with classroom foreign language learning, and other unholistic approaches to education. (p. 214, italicised as in original)

\subsection{Summary}

Vygotsky and his colleagues developed sociocultural theory to explain higher mental processes in interaction in a cultural context. Vygotsky's view of construction of mind has associated him with social constructivism. Vygotsky proposed a general learning and teaching pedagogy, which emphasises social interaction. However, he did not extend this pedagogy to learning a foreign language. The current research employs general sociocultural theory as a conceptual framework as originally developed by Vygotsky and extended by different sociocultural theorists. Details of sociocultural theory and its concepts interpreted and applied in classroom research are presented in the next chapter. 


\section{Chapter 3}

\section{SOCIOCULTURAL THEORY: INTERPRETATIONS AND APPLICATIONS}

\subsection{Introduction}

Having presented the origin of sociocultural theory, this chapter explores other interpretations and its applications in classroom research. Key terms such as mediation, artifacts, culture and affective factors will be discussed. In addition, the chapter provides the rationale for choosing sociocultural theory as a conceptual framework for the current study.

\subsection{Interpretations}

The publication of Vygotsky's works, namely, Thought and Language and Mind in Society has brought Vygotskian ideas to educationists and theorists worldwide. Thought and Language was originally translated into English by Eugenie Hanfmann and Gertrude Vakar and published in 1962. It was revised, edited, and translated again by Alex Kozulin in 1986 under the same title. In 1987, it was again translated by Norris Minick and published in The Collected Works of L. S. Vygotsky, volume 1 under the title Thinking and Speech. In the past decade, other works by Vygotsky have been translated into English and embraced with enthusiasm by many psychologists and educators. Sociocultural theory has been applied in different fields: psychology (Cole, 1996; Lave \& Wenger, 1991; Wertsch, 1985; Wertsch, del Rio \& Alvarez, 1995), pedagogy (Dixon-Krauss, 1996; Lee \& Smagorinsky, 2000; Tharp \& Gallimore, 1988; Wells, 1993, 1999), and second/foreign language research (Frawley \& Lantolf, 1984, 
Lantolf, 2000a, 2000b, 2002). According to Lee and Smagorinsky (2000, p. 1), the reason researchers have turned to sociocultural theory is that they had become dissatisfied with the stress on individual psychology:

This shift away from the study of the individual and toward the study of the social group and its cultural history highlights the role of social and material context in understanding how knowledge is both constructed and displayed.

Although referred to by different names: sociocultural theory (Cole, 1996; John-Steiner \& Mahn, 1996; Lantolf, 2002; Wertsch, 1998), cultural-historical theory (Bozhovich, 1977), and sociohistorical perspective (Dixon-Krauss, 1996, Matusov, 2001), the main focus of sociocultural theory is on mediation. The following section discusses different aspects of mediation.

\subsection{Mediation}

Daniels (2001), Lave \& Wenger (1996), and Wertsch (1998) considered mediation as the key to the Vygotskian approach to understanding how human mental functioning is tied to cultural, institutional, and historical settings, since the settings shape and provide the cultural tools that are mastered by individuals to form this functioning. The mediational means are what might be termed the "carriers" of sociocultural patterns and knowledge. For followers of Vygotsky, these are in the form of either a material tool (for example, tying a string around one's finger or using a computer); a system of symbols, notably language; or the behaviour of another human being in social interaction. Mediators, in any of these forms, transform natural, spontaneous impulses into higher mental processes, including strategic orientations to problem solving. 
Symbols, language and social interaction are all embedded in some context that makes them inherently sociocultural processes.

In the case of language learning, initially unfocused learning actions may become adjusted and modified based on how the learning of the language is mediated. This mediation can occur through a textbook, visual material, classroom discourse, opportunities for second language interaction, types of direct instruction, or various kinds of teacher assistance (Donato \& Bonnie, 1992; Engström, 1991; Tharp \& Gallimore, 1988). Mediated learning takes place when students act both directly on the sociocultural contexts and indirectly through mediational means, which may be both other humans (to transform information or daily meaning) and human made artifacts or other objects. Gillies (2003, p. 11) said that a person mediates when he/she "paraphrases to assist understanding, prompts, uses open questions in a tentative manner to promote thought about an issue the student is focused on, and engagement about an issue". Kozulin (1998) similarly considered mediation as the instrument of cognitive change. In the current study, mediation involves the use of language and artifacts to provoke speech and thinking in response to questions from the textbook or from other interlocutors.

According to Wertsch (1998), mediation offers both "affordances and constraints" in terms of how it empowers or enables action. Mediational means enable action and help in the understanding of action and the transformations it undergoes. However, mediational means also constrain or limit the forms of action we undertake. He quoted Gustfield: "culture and language not only open doors to experiences, they also form a prison which constricts and narrows" (in Wertsch, 1998, p. 40). 
Jordan (2003, p. 20) distinguished non-mediated learning from mediated learning: "In non-mediated learning, children interact directly with the environment, for example, in the form of observational learning, in what we would call trial and error, or free play. Mediated learning radically changes the conditions for interacting”. Lantolf (2000a) segmented mediation into three general categories: mediation by others in social interaction, mediation by the self through private speech, and mediation by artifacts. In the present study, social and artifact mediation are examined. The use of private speech occurred very infrequently and not often enough to warrant a separate category of mediation by itself. The language task took place within a group and most speech occurred as interpersonal interaction.

\subsubsection{Mediation Through Oral Language}

The primary function of speech, both for the adult and for the child, is the function of communication, social contact, influencing surrounding individuals. (Vygotsky, as cited in Barnard, 2000, p. 80)

Vygotsky (1962, 1978) considered that the nature of language is for social communication and that language develops entirely from social interaction. Language serves as a mediating tool for thought. External social speech was internalised through mediation and language in this sense is a tool for mediated learning. Other sociocultural theorists have interpreted mediation through language in different ways, however, with an emphasis on oral language. For example, Mercer (1995) claimed that Vygotsky described language as a psychological tool or something each of us uses to make sense of experience. Language is also a cultural tool which we use to share experience and so to collectively, jointly, make sense of it. The two functions of language, cultural 
(communicating) and psychological (thinking), are not really separate. Whenever we talk, we have to think of what to say, and think about what we hear:

Language is therefore not just a means by which individuals can formulate ideas and communicate them, it is also a means for people to think and learn together. Socio-cultural theory inevitably highlights the role of language in the construction of knowledge. Individually and collectively, we use language to transform experience into knowledge and understanding. (Mercer, 1995, p. 4)

According to Mercer (1992, p. 217), "An extremely important function of talk in the classroom is as a means for developing shared understanding" (italic in original). "The creation of shared knowledge and understanding is rarely, if ever, a matter of simply pooling information...it has to be generated by working with information" (Mercer, 1995 , p. 67 , emphasis in original). Talk could happen between teachers and pupils or pupils together. Mercer (1995, p. 104) discussed three kinds of talk in knowledge construction: disputational talk which is characterised by disagreement and individual decision-making; cumulative talk, in which speakers build positively but uncritically on what the other has said; and exploratory talk, in which partners engage critically but constructively with each other's ideas.

Wells (1999) considered that language is the tool for participants to engage in an activity together. Through dialogic inquiry, the less mature are helped to appropriate the culture's existing resources and guided as they use and transfer them for the solution of the problems that they consider important.

Candlin and Mercer (2001, pp. 6-7) argued that in sociocultural theory, "language is not only a medium for exchanging and constructing information but also a tool for thinking". Interactions among learners contribute to the emerging sociocultural climate 
when mutual interests are discussed and information and ideas are exchanged (Barnard, 2000, p. 113).

According to Wink and Putney (2002), the social nature of sociocultural theory has two senses: "One sense of social, then, is the interaction with others. Another sense of social has to do with the historical and cultural mediation of language used and produced between individuals" (p. 126).

Vygotsky (1978) originally used the term "language" in a broad way to cover both oral and written forms. By and large, sociocultural theorists have interpreted language as being restricted to speech exercised mainly in dyads or groups. "Sociocultural perspectives derived from Vygotskian theory tend to privilege verbal communication" (Mistry, 2001, p. 339). This gives the body of recent sociocultural studies a bias towards oral language and problems over whether or not students speak. It also accounts for the relative lack of interest in non-verbal language and the link between oral language and written language as presented in forms of artifacts, such as a textbook.

\subsubsection{Mediation Through Non-verbal Language}

In recent times, interest has been shown in non-verbal interaction in sociocultural theoretical perspective. However, this is true for only a few authors (McCafferty, 2002; Negueruela, Lantolf, Jordan \& Gelabert, 2003). Negueruela et al. (2003) argued that in social interaction, gestures complement speech to convey meanings, for example, hand moving as an accompanying illustration. "Gestures not only complement the meaning 
of language, but they also reveal the significance of an utterance for the speaker" (Negueruela et al., 2003, p. 4). These authors pointed out that gestures can serve both a communicative as well as cognitive function. They defined that,

In popular usage, "gesture" refers to the conventional manual movements that optionally co-occur with speech, such as, for example, waving to indicate leavetaking. (Negueruela et al., 2003, p. 2)

Negueruela et al. also considered that in speaking, gestures can simultaneously externalise a speaker's personal understanding of a situation for some other person and aid the speaker in developing this. McCafferty (2002) argued that the role of gesture in and of itself and in conjunction with speech creates zones of proximal development for second language learning and developing. The following section will discuss in detail the concept of the zone of proximal development.

\subsection{Mediation in the Zone of Proximal Development}

Sociocultural theorists have been interested in the concept of the zone of proximal development (ZPD), which Vygotsky (1978, p. 86) defined as,

The distance between the actual developmental level as determined by independent problem solving and the level of potential development as determined through problem solving under adult guidance or in collaboration with more capable peers.

The ZPD has been a springboard for different interpretations of mediation. In this section, only the most common interpretations are discussed. Based on the concept of the ZPD, teaching and learning have been interpreted as scaffolding (Wood, Bruner \& Ross, 1976), co-construction (Jordan, 2003; Rogoff, 1998) and assisted performance (Tharp \& Gallimore, 1988). 
Scaffolding is a metaphor for graduated assistance provided to the novice, akin to the builder's scaffold. The scaffolded help that the expert provides to the novice is characterised by six functions:

1. recruitment - enlisting the learner's interest in the task,

2. reduction in degrees of freedom - simplifying the task,

3. direction maintenance - keeping the learner motivated and in pursuit of the goal,

4. marking critical features - highlighting certain relevant features and pointing out discrepancies between what has been produced and the ideal solution,

5. frustration control - reducing stress and frustration during problem solving, and

6. demonstration - modelling and idealized form of the act to be performed by completing the act or by explicating the learner's partial solution. (Wood et al., 1976, p. 98)

Stone (1998) criticised the concept of scaffolding as a metaphor for learning and teaching for seemingly focusing exclusively on adults as the agents for instilling new understanding and for its assumptions about idealised adult-child relationships. Likewise, Matusov (2001, p. 16341) pointed out, "If Vygotskij's concept of ZPD seems to be too focused on the child, then the concept of scaffolding seems to be too focused on the role of the adult in guidance". Rogoff (1998) also criticised scaffolding for being too mechanical because children as well as adults are active and can manage their interaction. She further pointed out that,

The concept of scaffolding does not refer to the institutional and cultural context in which it occurs, whereas the concept of the ZPD requires attention to processes of communication and the relation of the interaction at hand to institutional, cultural, and historical processes." (p. 700)

Jordan (2003) proposed the concept of co-construction may be more appropriate than scaffolding, because working in a co-constructive environment, learners have the power to make decisions, their expertise is valued and the outcomes of their efforts are 
not pre-determined. A scaffolding environment, according to Jordan (2003), is aimed at specific outcomes and success is achieved through being able to ascertain what it is that the teacher wants them to learn or produce.

Also based on the concept of the ZPD, Tharp and Gallimore (1988, p. 21) considered Vygotskian pedagogy as assisted performance, "Teaching consists of assisting performance. Teaching is occurring when performance is achieved with assistance". In this pedagogy, children and youths are active engineers of their own learning and play, and teachers/experienced others have a key role in determining their next intellectual challenge. The role of teacher mediation goes beyond modelling or demonstrating how to do something. While the teacher is interacting with the student, he/she continuously analyses how the student thinks and what strategies the student uses to solve problems and construct meaning. Rather than being pre-planned, scripted or didactic, the teachers' conversations should be "instructional conversations" or based on the assumption that the students "may have something to say beyond the 'answers' already known by the teacher" (Tharp \& Gallimore, 1988, pp. 196-197). Teacher assistance is contingent upon students' responses. "Scaffolding involves not simply helping to do but helping how to do" (Gibbons, 2003, p. 249). In other words, in sociocultural theory, language is used as a tool in interaction and the teacher is the mediational tool.

The mechanism for growth in the ZPD is the interaction with a more experienced member of society. "More experienced" does not always mean an older person but can be someone of the same age or even younger, whoever has more experience and knowledge of certain fields. Furthermore, Wells (1998, p. 345) stated that the current view of the ZPD has been expanded beyond expert-novice interaction, "The ZPD as an 
opportunity for learning with and from others applies potentially to all participants, and not simply to the less skilful or knowledgeable."

The ZPD has been seen as an especially promising concept for organising teaching/learning activities, because learning in the ZPD is about developing an ability to engage with, and participate in, a particular environment, whether it be the classroom or another cultural setting. This means that the learners, with support from the teacher and other learners, must assume control of their own participatory activities (van Lier, 2000).

Hence, the ZPD concept emphasises collaboration in learning. It offers a set of pedagogical practices in which students are grouped and urged to work together in order to facilitate active involvement in discussing, explaining, critiquing and defending different perspectives on a common theme (Forman \& McPhail as quoted in Newman \& Holzman, 1993, p. 76). It may be seen as a process of which the constituent elements may be broken down as follows:

1. a 'problem' (a learning task) is identified by expert and/or learner

2. the existing knowledge or ability of the learner is ascertained

3. the inability of the learner to do the task without assistance is assumed

4. the learner's potential ability ('buds of development') to do the task is gauged

5. an intervention strategy is designed and applied by the expert

6. the learner's awareness, knowledge, skills and experience are activated

7. the learner manifests understanding of specific knowledge, understanding or skill

8. there is indication that the learner's understanding extends beyond the specific task (adapted from Barnard, 2000, p. 87).

Although the concept of the ZPD was originally developed as a pedagogy for teaching children, it has been applied with equal validity to learners of any age, in formal and informal settings and in asymmetrical (i.e. expert-novice) as well as symmetrical (i.e. 
equal ability) settings (Granott, 1993; Storch, 2002; Tharp \& Gallimore, 1988; van Lier, 1996; Wells, 1993). Additionally, the concept of the ZPD has been applied to foreign or second language learning (see section 3.2, this chapter).

Vygotsky himself started the trend. However, Wertsch and Rogoff (1984, p. 8) criticised Vygotsky for making several additional general comments about the ZPD but,

Nowhere in his writing did he provide an account of what constitutes problem solving under guidance or in collaboration with more capable peers. In the absence of such an account, the level of potential development, and hence the zone of proximal development in general, cannot be defined in any precise way.

Similarly, Daniels (2001, p. 59) raised questions regarding the "hints", "supports", or "scaffold", "Are they produced by the "more capable partner' or are they negotiated?" Daniels considered that Vygotsky is unclear on this matter. "The examples Vygotsky gave to demonstrate the use of the zone of proximal development suggest that he conceived of the environment as a static background to the dynamically developing child" (Van der Veer \& Valsiner, 1991, p. 343).

Ageyev (2003) argued that the ZPD has been interpreted, understood and further developed according to American cultural and intellectual traditions. However, originally, Vygotsky wrote about the concept from the collectivist point of view. Ageyev (2003, p. 14) commented,

The social interactions capable of creating the zone should bear some important characteristics typical for collectivistic and feminine cultures, such as profound emphasis on interdependence and the personal and interpersonal aspects of interaction. 
In short, sociocultural theorists emphasise the role of teachers, peers and learners and take into consideration social interaction. In a neo-Vygotskian view, the role of teachers and peers is that of a mediator to provide guidance, strategic support, and assistance to help learners assume control of their own learning.

\subsection{Peers and More Knowledgeable Peers}

Vygotsky's concept of the ZPD and the roles of the more capable peers in assisting learners to move beyond their potential developmental levels have inspired various studies of peer interaction. However, the roles of peers have not been well defined. Originally, the notion of peer in Vygotsky's perspective refers to children in classrooms. The literature to date shows different interpretations: peers as learners (van Lier, 2000), learners different from teachers (Swain \& Lapkin, 1998), more or less proficient learners (Ohta, 1995), more or less informed children (McDonald, Kidman, \& Clarke, 1991), peers as native and non-native speakers in classrooms (Barnard, 2002, Olmedo, 2003). Peers can be symmetrical or asymmetrical (De Guerrero \& Villamil, 1994). Peers are learners working jointly or collaboratively sharing the same goal (Lantolf, 2000a). Overall, the notion of peer has generally been associated with classroom learning.

From a sociocultural theory perspective, both expert-novice and peer interaction can result in mediation. Sociocultural theory supports the roles of adults/more knowledgeable teachers, and peers in mediated learning. Experts and peers are able to assist novices and other peers in the learning process through talk: scaffolding (Wood, 
et al. 1976) (see section 3.4), guided construction of knowledge (Mercer, 1995) and communicative symmetry (van Lier, 1996).

Guided construction of knowledge explains how language is used to create joint knowledge and understanding. It also explains how people help other people to learn and takes account of the special nature and purpose of formal education. Social mediation also happens in peer interaction. Communicative symmetry gives equal respect for the voices of the participants. Peers are often able to exploit the occasions for learning made available by their colleagues in ways they cannot in expert-novice interaction.

Lantolf (2000a, p. 84) argued that peers can mediate each other,

People working jointly are able to co-construct contexts in which expertise emerges as a feature of the group rather than residing in any given individual in the group...Expertise can be collaboratively constructed in the talk that occurs among learners who share the goal of working out a linguistically-based solution to a problem.

Two equally experienced learners can scaffold each other. A less experienced peer can actually self-regulate while working with a more experienced peer or can self-regulate while working by himself/herself with certain materials and tasks.

\subsection{Interpretations of Learning}

From all the interpretations in sections $3.3,3.4$ and 3.5 , it can be said that sociocultural theorists consider learning happens first through social interaction. It can happen through the mediating process, instructed guidance, and dialogic communication in joint construction of knowledge. Language in a sociocultural perspective is the primary 
mediational tool for learning and development. Learning at the micro level consists of face-to-face interaction and at the macro level the practices of culture. The resources for learning include materials and symbols that are available in activity settings and inherited from cultures. In neo-Vygotskian pedagogy, "On the whole, the emphasis is upon pedagogic contexts in which the practice is mediated by the students rather than mediated by the demands of the teacher" (Daniels, 1993, p. xviii). Practice in neoVygotskian pedagogy is socio-centred. Individuals in sociocultural theory when guided are active constructors of their own learning environment (Mitchell \& Myles, 1998).

Learning in sociocultural theory also occurs through collaboration. Students come together as a "community of learners" in which they are engaged in activities that facilitate development of all elements of communication (Brown, Metz, \& Campione, 1996; Jennings \& Di, 1996). They are also part of a "community of practice" in which individuals engage in and contribute to the practices of their communities (Wenger, 1998), and a "community of inquiry" (Linfors, 1999; Wells, 1999) in which learners learn with and from each others as they engage together to elicit another's help in going beyond their own present understanding.

Sociocultural theory provides a framework for pedagogy which places an emphasis on "joint activity" and "collaboration". Also, since making use of social resources is the way in which learning and development are interpreted, sociocultural theory promotes an orientation to the study of dyadic or group interaction. Sociocultural theorists' view of the student as an active participant in shared knowledge and meaning through social interaction is consistent with constructivism (see section 2.7, Chapter 2). However, 
sociocultural theory adds the cultural context of learning to the constructivist perspective.

\subsection{Culture}

Vygotsky (1978) considered that the social environment produces learning and development. The "social environment" embraces the concept of culture. Sociocultural theorists have interpreted the concept of culture in different ways. Cole (1998, p. 291) defined it as,

human being's "social inheritance." This social inheritance is embodied in artifacts, aspects of the environment that have been transformed by their participation in the successful goal-directed activities of prior generations. They have acquired value.

Luria (1979) saw the "cultural" aspect of Vygotsky's theory not only encompassing the learning tools of their society, but also the organisation and types of tasks children face when learning.

Cole (1996) additionally considered that culture and mind create each other. When language, signs and artifacts are objects in the environment, they are external but when they are in mind through the mediational process, they become the internal system of the mind.

Culture also includes the notion of cultural models, scripts, and the like (Cole, 1996). Cultural mediation implies a mode of developmental change in which the activities of prior generations are accumulated in the present as the specifically human part of the environment. This form of development, in turn, implies the special importance of the 
social world in human development since only human beings can create the special conditions needed for that development to occur. Culture in sociocultural theory, therefore, is not high culture or ethnic culture but lived experiences available to all of us.

\subsection{Tools and Artifacts}

Man differs from animals in that he can make and use tools. These tools not only radically change his conditions of existence; they even react on him in that they effect a change in him and his psychic condition. (Luria, 1928, p. 493)

Cultural tools and artifacts represent another aspect of sociocultural theory. Although the words "tool" and "artifact" are considered to be interchangeable (see, for example, John-Steiner, 2000; Moll, 1989; Wartofsky, 1979), tools include the full range of cultural means, such as language, mathematics and computers for use. Cole (1998) considered that it is through participation in such culturally structured activities that we become who we are. And who we are is defined by the ways we interact with others in culturally structured activities. Tools can be understood as historically accumulated artifacts, and the most important of all tools is language.

Artifacts, the constituents of culture, are simultaneously material and ideal/symbolic. They are materialised in the form of objects, words, rituals, and other cultural practices that mediate human life. They are ideal in that their form has evolved to achieve prescribed means to prescribed goals, and they have survived to be tools for our use, "partial solutions to previously encountered problems." (Cole, 1998, p. 292)

The literature of sociocultural theory shows different interpretations of mediation through artifacts. According to Moll (2000), human beings interact with their worlds primarily through mediational means, for example, the use of cultural artifacts, tools 
and symbols, including language which plays crucial roles in the formation of human intellectual capacities. Moll (2000, p. 265) argued that we have to seek culture in practices without detaching human beings from sociocultural dynamics, whether in classrooms, households, or other settings:

Human beings and their social and cultural worlds are inseparable; they are embedded in each other. Thus, human thinking is not reducible to individual properties or traits. Instead, it is always mediated, distributed among persons, artifacts, activities, and settings. People think in conjunction with the artifacts of the culture, including, most prominently, the verbal and written interactions with other human beings.

Cole interpreted Vygotsky's ideas of mediation, and defined the structure of artifactmediated action, in the following triangle:

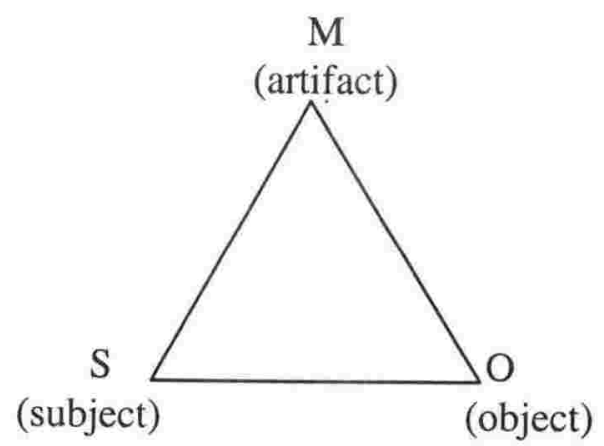

The basic mediational triangle in which subject and object are seen not only as "directly" connected but also simultaneously as "indirectly" connected through a medium constituted of artifacts (culture). (Cole, 1996, p. 119)

Cole pointed out that the "cultural" or "mediated" functions are those where the relation between subject and environment is linked through the vertex of the triangle (artifacts). Artifacts are the fundamental constituents of culture.

Cole (1996) acknowledged that it is hard to grasp language as artifactual, or handed out, but demonstrated it thus: 
When you hear a language that you do not understand, you are experiencing only its material manifestations. The meaning of the words, their 'ideal' aspect, is missing because you lack a past history of interactions of using those artifacts as mediational means.

On discussing artifacts, Wartofsky (1979) considered that there are three hierarchical levels of artifacts. The first level consists of primary artifacts, those directly used in production, such as axes, clubs, needles and bowls. This level corresponds closely to the concept of tool as it is ordinarily used. The second level, secondary artifacts, consists of representations both of primary artifacts and of modes of action using primary artifacts. An important kind of secondary artifact is cultural models, which "portray not only the world of physical objects, but also more abstract worlds such as social interaction, discourse, and even word meaning" (D'Andrale, 1984, p. 93). Similarly, Cole (1996, p. 130) pointed out that secondary artifacts include cultural schemas and scripts. Schemas, according to Cole, have generally been interpreted as mental structures inside the head. Schemas and scripts are the internal notion of culture as meanings. Second-level artifacts play a central role in preserving and transmitting modes of action. The third level is a class of artifacts that "can come to constitute a relatively autonomous 'world' in which the rules, conventions and outcomes no longer appear directly practical, or which, indeed, seem to constitute an arena of non-practical, or "free play or game activity"” (Wartofsky, 1979, p. 208). Wartofsky called these imagined worlds tertiary artifacts. Such imaginative artifacts, he suggested, can come to colour the way we see the "actual" world, providing a tool for changing current praxis.

Cole (1996) proposed that artifacts, such as books and texts are always involved in a dual mediation, with the nonhuman world and with other people. He considered "the 
concept of a tool as a subcategory of the more general conception of an artifact" (1996, p. 117). Tools and artifacts are embedded practices (Wells, 1999) which vary from culture to culture and from one setting to another so we must examine human existence in its social and historical aspects, not only its current surface (Tharp \& Gallimore, 1988) and make the study of surrounding social practices part and parcel of our inquiry (Cole, 1996).

In short, tools and artifacts are human cultural historical products. They include a range of material objects and non-material artifacts such as cultural practices and representations. They are used in an activity to assist and transform the activity and human thinking. An artifact may or not be a mediating tool. It requires a cultural context and an activity for human beings to use artifacts as mediating tools.

\subsection{Affective Factors}

Although sociocultural theory has been embraced by theorists as well as classroom teachers, it cannot avoid the criticisms that it lacks an emphasis on affective factors. Vygotsky acknowledged the social context of these external interactions; however, he paid inadequate attention to the instinctual/affective components of the internalisation process (Dean, 1994; Goldstein, 1999; Wertsch, 1985). Vygotsky did little empirical research on the affective aspects of consciousness and direct discussion of affect emerges infrequently in his writings. Vygotsky focused on the more strictly cognitive aspects of the process, leaving the affective nature of teaching-learning interactions unexplored (Goldstein, 1999). 
In fact, although Vygotsky paid some attention to affective factors, it is possible that his untimely death in 1934 prevented him from carrying out empirical research. The retranslation of his book Thinking and Speech has the following statement:

There exists a dynamic meaningful system that constitutes a unity of affective and intellectual processes. Every idea contains some remnant of the individual's affective relationship to that aspect of reality, which it represents. (Vygotsky, 1987 , p. 50)

In Thought and Language, Vygotksy resisted the separation of cognition and affect, emphasising instead "the existence of a dynamic system of meaning in which the affective and the intellectual unite" (Vygotsky, 1962, p. 8). Similarly, Vygotsky (as cited in Van der Veer \& Valsiner, 1994, p. 286) asserted that "the adolescent's emotional and intellectual aspects of behavior achieve their synthesis in his creative imagination". All of these suggest that he viewed cognition and affect as integrated and interdependent. However, he did not build this into his theory.

As for other sociocultural theorists and their focus on affective factors, Goldstein (1999, p. 654) pointed out,

Allusions to the role and the value of affect and interpersonal relationships in cognitive development can be found in the work of scholars who draw upon Vygotsky's theories; however, they are rarely considered in depth. Barbara Rogoff, for example, writes, "there is more intersubjectivity than just thinkingthinking, feeling, and emotion are all part of it" (1990, p. 9). In their Vygotskycentered book on scaffolding children's development, Berk and Winsler (1995, p. 29) begin a subsection with the heading "Warmth and Responsiveness" and state: "Another important component of scaffolding concerns the emotional tone of the interaction. Children's engagement with a task and willingness to challenge themselves are maximized when collaboration with the adult is pleasant, warm, and responsive. 
In recent times, more interest has been showed in affective factors. John-Steiner (2000) claimed that the facilitating role of social, participating others need not be limited to the cognitive sphere; it should take into account the process of appropriating emotional experiences. "Developing children, as well as developing adults, expand their affective resources by appropriating the consequences of shared experience. Such a process includes identification, scaffolding, expansion by complementarity, and constructive criticism" (John-Steiner, 2000, p. 128). John-Steiner (2000) and Mahn and John-Steiner (2002) pointed out that teachers can build on students' prior experiences, thereby helping them develop the confidence that engenders competence. Teachers can instil the gift of confidence in their students by offering caring support.

In a word, affective factors are not well documented in sociocultural studies. NeoVygotskian theorists did not discuss affective factors in depth, nor provide adequate evidence of empirical research. However, they have focused on the support of cultural resources.

\subsection{Cultural Resources}

Sociocultural theory advocates the connections between schools and the broader cultural contexts where learners live. The ability to communicate in and outside of the classroom is anchored in the social and cultural life of students, active engagement in learning activities, and ability to create social relationships with informed and competent persons (Cole, 1996; Dalton \& Tharp, 2002; Moll, 1990; Wertsch, 1985). Dalton and Tharp (2002) proposed three levels to connect school to students' lives. According to them, at the first level, it is necessary to invoke students' existing schema 
and the content of instruction should be drawn from students' own environments and experiences. The second level should embrace advocacy for instructional use of cultural artifacts as the media in which goals of literacy, numeracy and science are conceptualised. Drawing on personal, community-based experiences afford students opportunities to apply skills acquired in home and school contexts. And at the third level, there are advocates for contextualization of the school itself. School learning is a social process that affects and is affected by the entire community.

Moll, Amanti, and Gonzalez (1992) proposed the concept of "funds of knowledge" to refer to the strategic knowledge and related activities essential in households' functioning, development and well-being. Moll et al. argued that the teachers should not only know students from their performance within rather limited classroom contexts. Classrooms should not be considered encapsulated from the social worlds and resources of the community because,

The cultural structuring of the households, whether related to work or play, whether they take place individually, with peers, or under the supervision of adults, helps constitute the funds of knowledge children bring to school. (Moll et al., 1992, p. 134)

In a similar vein, Wink and Putney (2002, p. 97) considered that cultural resources, household activities and wider communities play a role in the learning process through "funds of knowledge that are grounded in the networking that communities do in order to make the best use of those resources". Moll $(1990,2000)$ also demonstrated the importance of communities of learners within large cultural and familial networks, and argued that schools should draw on the social and cognitive contributions that parents and other community members could make to children's development. 


\subsection{Applications of Sociocultural Theory in Classroom Studies}

In this section, classroom studies within a sociocultural theory framework are presented.

Peer or expert-novice interactions have been the focus of the following sociocultural studies. Tudge, Winterhoff, and Hogan (1996) found that children whose partner exhibited higher-level reasoning were far more likely to benefit from collaboration than those whose partner did not. Swain (2000) examined mediation from the perspective of collaborative dialogue in a French immersion class. She argued that collaborative dialogue is a key form of mediated learning. The study demonstrated how young learners, through collaborative dialogue, are able to organise and mediate their own learning without the intervention of the expert teacher. Native speakers and non-native peer interaction is a critical component of second language development. Barnard (2002) studied peer tutoring in the primary classroom. The finding showed that pupils can scaffold the learning of their classmates and especially those came from nonEnglish speaking backgrounds by explanation, exemplification, demonstration and modelling. Olmedo (2003) similarly found that native speaker peers used strategies, for example, translation of a message, paraphrasing of a message, code switching and making gestures to accompany speech to scaffold communication with non-native speaker peers in the classroom.

Scaffolding has been used to address a variety of aspects of language learning from the acquisition of lexis to the development of learning strategies. Van Lier (2000) found that learners can scaffold their peers' learning, and exploit the "affordances" or 
"occasions for learning" made available by their colleagues in ways they cannot in expert-novice interaction. A study by Donato (1994) explored the process through which adult foreign language learners mediate each other through collaborative interaction in a classroom learning environment. Donato found that collective groups are able to construct jointly the scaffold necessary to complete a learning task. However not all students work collaboratively when assigned to work on language tasks in pairs. Scaffolding is more likely to occur when pairs interact in a certain pattern: either collaboratively or in an expert/novice pattern (Storch, 2002). The teacher could scaffold the learners through such communicative moves as directives, assisting questions, repetition, and nonverbal devices such as pauses and gesturing (Anton, 1999). The process helped the teacher succeed in involving students in negotiating meaning and form, as well as classroom behavioural rules.

It has also been shown that peers in ESL classrooms can scaffold learning (Donato, 1988; Ohta, 1995; Swain \& Lapkin, 1998). Donato (1988) found evidence of "collective scaffolding" in groups in which members drew on their resources and together scaffolded their resolution of language-related problems they encountered. The study by Ohta (1995) showed that peer interaction allows learners to act as both expert and novice, constructing their roles through the varying levels of expertise. Ohta also argued that in the collaborative learning situation, students are able to assist one another through scaffolding to accomplish tasks beyond their current level of linguistic competence and beyond what they are capable of without assistance. Swain (1995) found that dialogues among learners can be similar to instructional conversations between teachers and learners. Peers in pairs can use language for meaning-making activity both within and beyond the context of the assigned role-play task. 
Sullivan $(1996,2000)$ focused on the role of playful behaviour in mediating the interaction that unfolds in an adult EFL classroom setting. Sullivan suggested that the discourse in an EFL Vietnamese classroom is like that of a family where individuals build upon each other's ideas in a humorous way.

Sociocultural theory was originally applied in research involving a first language. However, it has been employed in research in the second/foreign language classroom. In this trend, studies of learner interaction in EFL/ESL have not paid much attention to the use of the first language. The literature shows a small number of studies in collaborative interaction analysed in a sociocultural theory framework with a discussion on the use of the mother tongue (e.g., Anton \& DiCamilla, 1998; Brooks \& Donato, 1994; Swain \& Lapkin, 1998; Villamil \& DeGuerero, 1996).

Anton and DiCamilla's study (1998) in the functions of the first language in collaborative interaction in the second language classroom demonstrated that learners of Spanish used the first language as a means of scaffolding learning. The authors argued that the first language "provides an opportunity for second language acquisition to take place" (p. 322). Anton and DiCamilla (1998) also pointed out that from a sociocultural perspective, the use of the first language is beneficial for language learning since it acts as a critical psychological tool that enables learners to construct effective collaborative dialogue in the completion of meaning-based language tasks by performing three important functions: construction of scaffolded help, establishment of intersubjectivity, and use of private speech. The use of the first language provides a means to create a social and cognitive space in which learners are able to provide each other and themselves with help throughout the task. Brooks and Donato (1994) studied 
Spanish speaking learners who were learning English. They found that the first language is an important component of discursive activity in both initiating and sustaining discourse. It is used in metatalk, or talk about the task at hand, and the discourse that constitutes the task. Metatalk serves to promote verbal interaction and is one type of verbal metacognition. Villamil and De Guerero (1996) studied adult learners whose first language was English and who were learning Spanish. The finding showed that student collaborators used the first language to provide scaffolding, and to vocalise private speech. Swain and Lapkin (1998) compared metatalk performance of French immersion students across two different tasks. They found that lower proficiency students with English as the first language relied more on their first language than higher proficiency students. Although research on the first language use to mediate collaborative activities has uncovered important insights, it has not fully explained why learners opt to rely on their native language (Lantolf, 2000a) and what are the outcomes if they do. The research reported in this thesis was built on these studies with a focus on the use of the first language, Vietnamese, as mediation in group discussion.

With regard to research on non-verbal interaction, McCafferty's (2002) research on gesture and language learning revealed that gesture played a part both in promoting language learning and in facilitating positive interaction between the participants, helping to create a sense of shared social, symbolic, physical, and mental space. Gestures are contextual cues to support comprehension and act as mediators, for example, raising hands to indicate that the children are ready to begin an activity (Olmedo, 2003). The research by Kellerman and van Hoof (in press as quoted in Negueruela, et al., 2003) and Negueruela et al. (2003) found that the first language 
learners showed a strong tendency to transfer their first language gesture-speech patterns to their second language English performance or rely on first language gestures in learning another language.

Sociocultural theory has been criticised for placing too much emphasis on verbal interaction (Mistry, 2001). This seems justified, as there has been little regard to the roles of artifacts in learning as shown in classroom research within a sociocultural theory framework (see for example, Anton, 1999; Forman \& Cazden 1985; Gibbons, 2001; Mercer, 1995, 2001; Moll \& Whitmore, 1993; Palincscar \& Brown, 1984; Swain 2000; Wells, 1999). These studies were conducted at different times and in different contexts. They provided a brief introduction of what was supplied to the students and then began with analysis of the transcriptions. All of the studies came to the conclusion that talk between the teachers and students or among peers could help the learners to be able to do the tasks that were beyond their level of competence. However, the discussion of findings did not give an explanation of how the artifacts and tools, such as a textbook, a worksheet, a computer programme, or a toy car were used to create talk. Artifacts seem to be considered as a backdrop to oral language mediation although they are often examples of language in written forms.

Other researchers have investigated the roles of artifacts in the science classrooms. For example, van Boxtel, van der Linden, and Kanselaar (2000) carried out a study on the mediational role of textbooks in physics learning. The research findings showed that the textbook had a negative influence on the amount of elaboration and co-construction in the student interaction. Hill, Cummings, and van Aalst (2003) studied the mediating artifacts such as electric circuit boards, electricity concepts, and Knowledge Forum 
database (a resource in which ideas and questions are recorded in words as well as in pictures and discussed) in a science class. They found that artifacts structured participation in the knowledge building discourse. Contributions to the discourse are shaped by the particular artifacts that students were using. Schwarz and Hershkowitz (2001) explored the role of computer artifacts, the representative, in mathematics classrooms. They found that "the construction of meaning of mathematical functions is described as a process that occurs through social interaction and the interweaving of the ambiguous computer-based artifacts" (p. 250). Nevertheless, even the studies in science classrooms have generally been concerned with students' talk when using the artifacts (Sfard \& Kieran, 2001) rather than with exploring the meaning transmitted by the artifacts themselves; that is, their function as mediating tools.

Although a great deal of empirical classroom research using sociocultural theory as a framework has been carried out, very little has focused on affect in face-to-face social interaction. The study by Mahn (1997) seems to be the only one using sociocultural theory as a framework to investigate students' emotions using dialogue journals. The findings showed that students revealed their anxiety through frequent reference to their fear of making mistakes. This anxiety inhibited their writing and caused further frustration as they hindered their ability to communicate their ideas. However, the study also showed that students gained confidence through dialogue journals, and their writing was transformed, as it became a vehicle for self-discovery. The narrative fluency that helped students forge writing identities also helped them to develop ideas more completely in academic writing. The collaborative aspect of the journal writing and the creation of a relatively risk-free environment also carried over into the course as a whole and contributed to the construction of a collective ZPD. Overall, the 
students gained confidence which was developed through the genuine support in the teacher's responses, and which helped them to express ideas and emotions that they might not otherwise have attempted.

As seen from the discussion in this chapter, there are some gaps in the literature of sociocultural theory. Firstly, there is no discussion of the relation between oral interaction and the artifacts in the form of written language, for example, a textbook. Oral interaction may not be the only means of learning in every culture. Secondly, the affect in the learning processes is not comprehensively documented or studied. Thirdly, culture as part of emotion has not received attention. Fourthly, each of the previous studies using a sociocultural theory framework tended to focus on a particular aspect of sociocultural theory, whether mediation through language, artifacts as mediation, or mediation in cultural contexts. While all these aspects complement each other and can mediate the learning processes, no sociocultural studies have been set up to investigate all of these factors in one study. Fifthly, none of the previous research reviewed above compared and contrasted what happens in group work among students from the same class with group work with peers and a student from a more advanced class, which this research addresses. Lastly, despite the volume of interpretation of sociocultural theory, there are some unanswered questions, for example, "What is a peer?", "How does the composition of a group influence the interaction of peers?" and "How do peers work in different group settings?" The current study was built on the previous research but looked to a fuller picture of learners in both face-to-face interactions in groups in the classroom and in their broader cultural context to see what opportunities are available for them to learn English outside the classroom. In addition to investigating the role of peers and languages (both English and the mother tongue, Vietnamese), it aimed to find 
out more about aspects of mediation through artifacts, affective factors in group interaction, the links between cognition and emotion, and between emotion and culture.

\subsection{Sociocultural Theory and EFL Approaches}

Viewed in EFL approaches, sociocultural theory holds a strong interactionist view of language learning (Le, 2003a). There is a difference, however, between the sociocultural perspective and other perspectives that also view interaction as important in language learning. Interactionist models assume that input modification provides learners with linguistic raw material that they will then process internally and invisibly (Lightbown \& Spada, 1999). In the interactionist view, language input must be modified and comprehensible. Thus, the use of group work in the language classroom has long been supported because group interaction provides a rich language input environment. Group work increases the quality and quantity of student talk, individualising instruction, and creating positive affective climate in the classroom (Long \& Porter, 1985, pp. 207-212).

Negotiation is used in second language learning as the process of interaction for input and output. Negotiation in the current research means the process in which "speaking is used as a strategic tool for cognising and constructing tasks, meaning, and shared situational definition" (Brooks \& Donato, 1994, p. 263). In other words, the role of speaking is a cognitive activity (Vygotsky, 1986). Brooks and Donato (1994, p. 266) further pointed out that in sociocultural theory speaking has mediational functions which

Do not neglect the learners as co-constructors and sense-makers of their own interactions with respect to the task and to each other. Moreover, speaking and 
thinking are closely linked and are seen as co-constituting the total activity of the learners.

From a sociocultural perspective, learning goes beyond providing a rich language environment for learners. Learners and teachers use the language as a cognitive tool to develop thoughts and ideas. As Gibbons (2003, p. 248) put it, "Sociocultural theory views language learners not as processors of input or producers of output". Teaching and learning language in the $\mathrm{ZPD}$, teachers and more capable peers must pay attention not only to language input but also to language learners (Lantolf, 2000a, p. 80) because they have to provide the learners with a supportive and guiding environment in addition to knowledge that is beyond the learners' current level. With an emphasis on social learning and mediational roles of cultural and linguistic artifacts as well as teachers and peers, sociocultural theory promises a profound theoretical base for teaching English as a foreign language (Le, 2002b).

\subsection{Sociocultural Theory as a Conceptual Framework for the Current Study}

If learning another language is mediated by the social environment of the classroom and if, in addition, this social environment is supposed to lead to the reorganisation of sociocultural consciousness through the acquisition of foreign cultural meanings, then we need much more research into the social context of classroom FLL [Foreign Language Learning]. (Kramsch, 1990, p. 31)

The study described in this thesis is a classroom study.

The findings of classroom research need to be embedded in an evolving explanatory theory of classroom learning that is of practical value to teachers.

(Nuthall \& Alton-Lee, 1990, p. 547)

Sociocultural theory was chosen as the conceptual framework for the current study for the following reasons: 
Firstly, a fundamental idea in Vygotskian approaches to learning is that knowledge and learning are constructed in the mind of the learners in a social or cultural context. This social construction of knowledge is mediated by language and social activity. Language is a cognitive tool whose acquisition enables learners to gain, process, organise and evaluate knowledge. It is also a cultural tool, by which knowledge is shared, stored, and made available to successive generations, and a pedagogic tool by which intellectual guidance is provided to learners by other people (Mercer, 2001). This fits in with current emphases in education on interaction for learning, collaborative approaches, and recognising the importance of the social context and interaction in the classroom.

Secondly, "the perspective of sociocultural theory is able to integrate levels of analysis from the macrolevels of culture to the microlevels of social interaction and individual thinking and speech" (Panofsky, 2003, p. 19).

Thirdly, despite an increased interest in socio-cultural theory worldwide, there is very little empirical research that has actually been conducted from the perspectives of teaching and learning English as a foreign language. Vygotsky's theory is essentially concerned with teaching-and-learning, rather than joint learning. Neo-Vygotskian research involves either studies of asymmetrical teacher-learner relationships, or symmetrical relationships among learners. The current study focused on both interaction among peers and between a senior (or a more advanced) peer and learners.

Finally, sociocultural ideas about knowledge construction provide a way of analysing learning situations. As Confrey (1995, p. 212) points out, "Vygotskian theory emphasises the importance of the sociocultural context in which learning takes place 
and how that context has an impact on what is learned". The original feature of this research is to set up two different kinds of groups: unassisted and assisted, and explore the similarities and differences between them.

The components of sociocultural theory, namely, mediation through oral and written language, artifacts as mediation, interdependence and co-construction processes of joint learning, cultural tools, and community support will be used as analytical tools in the current research. Chapter 4 will provide a research diagram to show the relations of these components and the unit of analysis.

\subsection{Summary}

This chapter continued on from Chapter 2 by discussing how sociocultural theory has been interpreted and applied in classroom research. The focus was on mediation at different levels, such as mediation through oral language (the target language and the mother tongue), expert-novice mediation, peer mediation, and artifacts as mediation. The chapter also discussed the role of the wider community as a source of support and knowledge for learners. The gaps in the literature were identified. These gaps include the lack of research on the roles of peers and how peers work in different settings, the link between oral interaction with written artifacts, the relation between learning and emotion, and culture and emotions. The chapter also provided a rationale for choosing sociocultural theory as the conceptual framework for the research. The current study looks to sociocultural theory for research design and data interpretation. The next chapter will present the research design and methodology. 


\section{Chapter 4}

\section{RESEARCH METHODOLOGY}

\subsection{Introduction}

This chapter presents the research design and details about the pilot and main study. It describes the research methodology and methods of data collection, namely audiorecording, video recording, journal writing, and interviews. In addition, the chapter includes information about the coding scheme, analytic categories, and data analysis procedures. Finally, the chapter discusses the validity and limitations of the research.

\subsection{Research Design}

As pointed out in section 1.2, Chapter 1 , there were two aspects to the current study. The primary focus was to use socio-cultural theory to underpin the research questions and data interpretation. The second was to observe the differences in two pedagogical settings when a more experienced student was introduced into a group of students engaged in group work. A comparison of unassisted groups and assisted groups aimed to provide insights into the roles of peers and the variable nature of group work. The following research diagram shows the interrelation of all sub-units of analysis in the framework of sociocultural theory and the methods of data collection. 


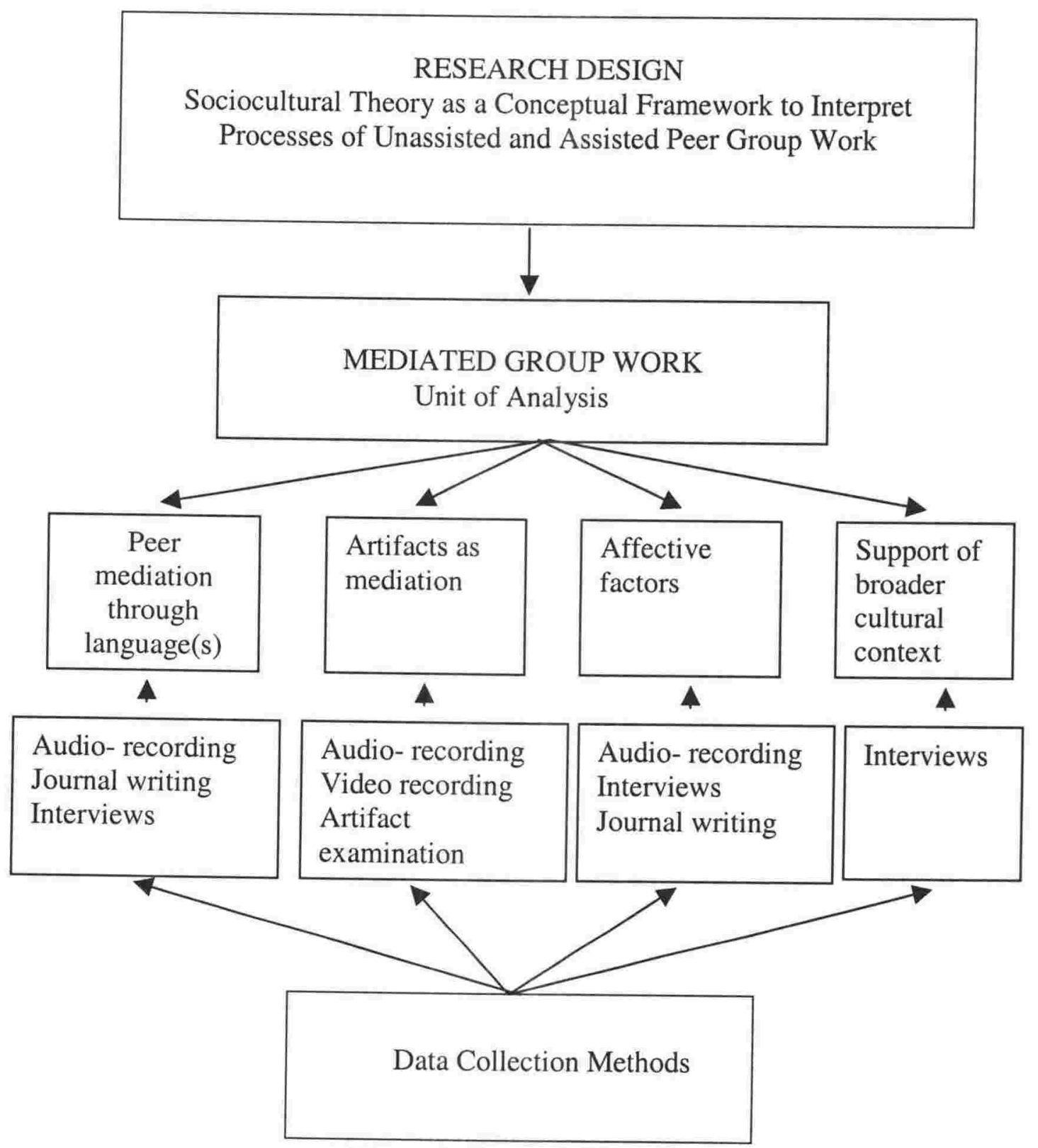

Figure 1: Research Diagram

The unit of analysis of mediated group work matched the focus of the research. It allowed comparison of group processes between the two kinds of groups: Unassisted and assisted peer groups. With this unit of analysis, it was also possible to examine groups at work within each kind of group condition (unassisted or assisted). Each subunit of analysis reflects an aspect of sociocultural theory as discussed in Chapter 3 and the research questions in section 1.2, Chapter 1. For example, the sub-unit of peer mediation through language(s) focused on interaction in group work. The artifact sub- 
unit explored the role of the textbook and other classroom materials. The affective factors unit examined the students' feelings during group work. The last sub-unit studied the environment for learning English in Vietnam. The data collection methods, as shown in Diagram 1, are discussed in detail in section 4.10.

I began my research with several ideas in mind. I wanted to introduce sociocultural methods into EFL in Vietnam and to find a better way of conducting group work. I also wanted to make a contribution to socio-cultural theory. First I identified in the literature some issues relating to sociocultural theory which had been applied in classroom studies, including studies of language learning (see section 3.11, Chapter 3), which did not appear to have been explored. For example, I could not find studies which compared peer groups according to their composition, and although there were some studies which included artifacts these were mainly in studies of mathematics and science. Then I tried to build a framework for the current study which would allow me to examine these topics. The subunits of analysis in the research diagram (see Figure 1) show how I built into my research design, a capacity to explore the topics I had identified.

I then returned to Vietnam for the fieldwork. The data collection in the classroom turned out to be a complex process. Learning depends on more than cognition and social interaction. After the pilot study, I realised I had to juggle the differing requirements and make decisions about what to look at. The students used artifacts during the group work which motivated me to look more into the issue of artifacts as mediation. However, I could not video all the processes of learning in groups because the students sat on fixed benches and I had to video record each group in intervals of 
five minutes. I then relied on audio recordings to look for more evidence of how artifacts influenced students' discourse in the two group settings.

I was afraid at first that the audio recordings would not give me good data because the class was crowded. However, the evidence gathered by audio-recording was the richest. The students were very enthusiastic about writing in their journals and they came to the interviews as true research participants, responsible for being on time, and eager to talk to me.

I might have been able to obtain stronger evidence of development in learning English if I had been able to observe the students for a longer period and had recorded them more often. However, a university term in Vietnam lasts only four months, followed by three months of summer break. I had to return to New Zealand to continue my $\mathrm{PhD}$ journey.

\subsection{Qualitative Approach}

Due to the nature of the research in a holistic and naturalistic classroom environment, qualitative research methods (Anderson, 1998, Firestone, 1993; Denzin \& Lincoln, 1994) were used. Creswell (1994, p. 2) defined qualitative research as

An inquiry process of understanding a social or human problem based on building a complex, holistic picture, formed with words, reporting detailed views of informants, and conducted in a natural setting.

In a qualitative study, the participants can be seen as whole persons in connection with the environment of the classroom and the wider society. Classroom practices cannot be 
understood without observing and talking to students first or letting them have an active voice in the process of inquiry.

Another reason for using a qualitative approach is that it emphasises many coincidental actions by looking at a wide sweep of contexts: temporal, historical, political, economic, cultural, social, and personal (adapted from Stake, 1995). These perspectives provide and deepen broader contexts and factors in connection with the research participants. In this particular study, the temporal context was embedded in the processes of group work. The social, cultural and historical contexts were used to interpret the data. As pointed out in section 2.4, Chapter 2, in sociocultural theory, human activities can be best understood when investigated in their sociocultural contexts. Political, economic and personal factors were also used to interpret students' motives to learn English.

Qualitative research is criticised for its subjectiveness. In this research, several strategies were used to enhance robustness (see section 4.13).

\subsection{Ethical Considerations}

An institution of higher education in Vietnam was chosen for the fieldwork for both the pilot and main study. Ethical approval was sought from the Ethics Committee of the School of Education, Victoria University of Wellington. The provisional working title of the research as stated on that application was changed subsequently to reflect the study more accurately (see Appendices A-E). Ethical approval was also sought from the director and university board of the institution in Vietnam. The research 
information sheet and consent form, written in Vietnamese, were submitted to the board of the department in which the study was carried out and the university rector in Vietnam asking for permission to access the site and to work with the students. Teachers' information sheet and consent form were written in English. Students' information sheets and consent forms were written in Vietnamese to make sure that the students understood clearly the procedure of voluntary participation. The English translations are included in Appendices B-F. The information sheet provided the background, general purpose, and the procedures of the research in which the students would participate. The consent form was attached to the information sheet asking for the signatures of the students as a proof of their voluntary participation. All the students approached agreed to participate.

When collecting the data, I assured the students of confidentiality, and confirmed that the research would not harm their marks or grades. I kept in mind that I was not a classroom teacher and I could not require them to do any task. I was aware that they were helping me to do the research. I treated them with respect and dignity at all times. Concerned that my relationship with them should not influence their responses in the journals and interviews, I assured them that my presence was not that of an authority figure or a classroom teacher. The student information sheet informed them of the purpose of the study but not the specific objectives, "My research is to investigate the learning and teaching of English as a foreign language at university level in Vietnam in the framework of sociocultural approach developed by Vygotsky and his followers." (Appendix B). I did not describe the specific objectives of the research, for example, the languages they used in the group discussion, the ways they referred to the artifacts available, or their gestures during the discussions. I was not too specific about the exact 
focus of my study, because awareness of this could affect the students' behaviour. I also had a formal talk with all the students in the class before the data collection about:

1. Their involvement in group work;

2. The presence of an observer (me) with a video camera;

3. The presence of a senior student working with the students in one kind of group;

4. The use of a tape recorder during group work, and that the students would wear a microphone;

5. The interview with me of about 20 minutes for each participant with a tape recorder; and

6. The confidentiality of any data gathered during the study and the removal of personal identifiers in any published documentation.

In Vietnam, a class period lasts 45 minutes. Between class periods, there is a break of five minutes. I arrived and left the class during break time to set up and put away the video camera and cassette recorders. I collected journal entries on the next day and also during break time.

Concerned that the video camera and microphones would interfere with the learning processes as well as the students' reaction to the research, I assured the students that the camera and cassette recorder were to record their natural behaviours and speech. I suggested that they should act naturally and avoid pretending to be serious. The audio transcriptions showed that the students spoke naturally. The laughter and the jokes (see section 10.2, Chapter 10) suggested that the students were behaving naturally during the learning process under observation. 
In their interviews and journal entries, only two students reported the influence of cassette recorders and the video camera, which made them nervous or shy.

Usually I feel comfortable but today I was recorded so I was not natural. (01U-J)

I felt stressed because it was the first time I contacted many strange things. (03U-J)

Because qualitative research seeks to understand the world through the eyes of the participants (Anderson, 1998), I was careful to make sure that the participants felt comfortable enough to speak with me. During the interviews or when I talked to the students, I tried to ensure that the students were comfortable. To create rapport, I 
4. To clarify any ambiguities or misunderstanding of the questions in the journals and in the interviews.

The pilot study was carried out with a different class but at the same level and in the same institution as the class in the main study. Three observations with video and audio recordings were made. The recordings operated well and did not seem to interfere with the classroom process. Nine students were interviewed and twenty journals were collected. It took the students about 15 minutes to complete a journal entry. The students did not have any difficulties in understanding the questions in the journals and interviews. The pilot data were analysed and some changes were made. Two journal questions were reworded so that they were less direct than the original ones as the students were from a culture in which people prefer to communicate in an indirect way. The two original journal questions:

-If the senior student was not helpful to you, please explain why not. -If you did not enjoy the group work today? Please explain why not and what in particular you did not enjoy?

were reworded as followed,

-What do you think are the disadvantages in having a senior student in the group work today? Please explain and give examples.

-In your opinion were there any disadvantages in the group work today? Please explain and give examples.

The original interview question: "Do you like to have learning and teaching aids, for example, overhead projector, cassette player in classroom? Why or why not?" was 
changed into "What learning aids do you have at your home? What learning aids are there in your classroom? Which learning aids do you find particularly helpful?"

\subsection{The Class Unit of Work}

The purpose of the class unit was to practise English speaking skills. The students were encouraged not to use Vietnamese. The teacher at the beginning of each discussion reminded all members that they had to speak and use English. The textbook Well Spoken by Ramsey and Rees-Parnal (1989) was the assigned course book for this class. Each student had a textbook either in English or in English and Vietnamese. It was up to each individual student to buy his/her own textbook. Some students shared their textbooks with classmates. The following is an extract from the introduction to the textbook, referring only to the topics covered by the students in the research (Ramsey \& Rees-Parnal, 1989, pp. 4-5).

\section{Topics}

Rural and urban

Good luck and bad luck

Advertising

Traditional and modern

Becoming an adult

Animals

\section{Thematic areas}

Description of transport, atmosphere and leisure time in town and country; influence of surroundings on health-stress, the homeless in cities; village, town and city life.

Omens of good and bad luck; ways of wishing people luck.

Effective advertisements, describing products for advertising purpose; banning advertisements.

Describing different styles; atmosphere; rooms and furniture; changing tastes.

The process of growing up; the landmarks of adulthood, attitudes to adulthood.

Comparing how people keep animals; different kinds of animals; your ideal holiday. 
Festivals and holidays

Work

Anger

Taking risks
Different kinds of festivals and holidays; the pros and cons of holidays; your ideal holiday.

Working styles in different societies; talking about work; value of jobs; attitudes towards work.

Describing anger; proverbs about anger; expressing emotions; giving advice on anger; personal experience.

Everyday risks; insurance; professional risk-taking; risk and responsibility.

These were the broad aims of the topics. The required task of each group session will be presented at the beginning of the analysis of sequences of the audio transcriptions.

The authors of the book also told classroom teachers and students about that the aim of the textbook as follows:

The aim of the material in Well Spoken is to motivate pre-intermediate learners of English to take part in conversations and discussions of various lengths and levels, about things that are either within their experience or that they can speculate about...The long-term aim of Well Spoken is to increase learners' fluency...The amount of preparation depends mainly on the learners' language level, their familiarity with the necessary vocabulary, their experience of activities which emphasise fluency rather than accuracy, and their willingness and ability to use the language they know spontaneously. Most of the responsibility for making the discussions successful lies with you, and not with the teacher. Try to say as much as you can about the topics, and always try to relate the topics to your own personal experiences and your opinions. (Ramsey \& Rees-Parnal, 1989, p. 7)

The university curriculum book for teaching English in Vietnam in use at the time of the current study, stated the objectives of the English speaking course:

Learners will be able to form necessary questions in verbal interaction. Learners practise discussing life in the countryside and the city, old people, advantages and disadvantages of different types of family, their points of view towards life, working styles, social evils, etc. by using the vocabulary and sentence structures they have learned. (University curriculum book for teaching English, 19961997, pp. 18-19) 


\subsection{Participants in the Main Study}

\subsubsection{The Students}

The institution where the research data collection took place was a degree-granting university in Vietnam. Students came to the university from different parts of Vietnam to obtain a bachelor's degree. They studied for four years. The student participants consisted of a class of 45 Vietnamese students (37 female and 8 male, aged 18 to 20 years) who were studying English as a major. They were in their first year of university study but they had studied English in high school. To be able to major in English, they had had to pass examinations in three subjects: mathematics, Vietnamese literature, and English. The total score for the three exams was summed. In the total score, the result for the English exam was counted twice. Their English proficiency was about "preintermediate level" in Vietnam, but there was no international comparison of the English proficiency of the students. They were in English classes for about 15 hours per week, with separate classes in English reading, writing, grammar, speaking and listening. On average they studied for four 45 -minute periods a day in class. A semester lasts four months. All of the student participants were Vietnamese nationals. They were all doing well with their English learning and there was no suggestion that the study was concerned about their standard of English. The students had been in the same class for one semester.

The data collection was carried out at the beginning of the second semester of the academic year 2002 in a class different from the classes in the pilot study, fitting into the programme of the academic speaking module for the second semester. The students 
met weekly. For each English speaking class session, a period of approximately 20 minutes was spent practising oral English in groups as part of the normal routine. Only two groups were observed during each class session, while the other students were working in groups in the same classroom. One group consisted of five students working by themselves (the unassisted group) and a second group consisted of four students together with a senior student from a higher class (the assisted group). For each observation, different students were recorded, but each student experienced the two different conditions. For each session of either kind of group, there was only one male student and four female students, which meant that some male students repeated their group work and recorded three times during the period of data collection. Some female students were also recorded three times to make the total of five students for the two groups under observation when the students whose turn it was to be observed were not available. The students were randomly assigned to groups. The senior student was introduced for the purpose of the study. Twelve observations were carried out during the course of the study.

All 45 students of one class volunteered to take part in the main study. By the time of the actual observations, the participants in the main study and I had had some acquaintance through informal and formal talks and the returning of consent forms.

\subsubsection{The Senior Student}

The senior student was in her fourth year at the same university and was therefore three years more senior than the participant students. Before my field trip to Vietnam, I sent some email letters to her class to ask for volunteer research participants. She was the 
one who showed great interest in the research. She asked me about the procedures and how to participate as a senior student. Being a fourth year student at university, she had had the experience of doing a mini research project. She communicated very well in English through her emails to me. I decided to invite her to be the senior student. She agreed and we kept in touch by email. The senior student was not a trained teacher and she was not trained for the purpose of the research. When I met her in Vietnam, I had informal talks with her about the procedures of participating in the research. I informed her of her role in the group work as a group member and advised her not to adopt the role of an authoritarian person. I introduced the senior student to the student participants to have an informal talk and to become acquainted. I assured the student participants that she would work with them as a group member and a participant. This approach avoided, to some extent, concerns raised by Confrey (1995) that the more experienced "other" may use indoctrination to achieve the convergence of a learner's activity to his or her own educational approaches. I was concerned that the senior student may encounter uncooperative students in groups but none of this behaviour occurred.

In Vietnam, students stay in the same class for the whole degree programme. Students gather only half a day at university. Different level classes study at different times in a day, whether in the morning from 7 am till 11: 30 am or from $1 \mathrm{pm}$ till 5:30 pm in the afternoon. The availability of a senior student was thus possible.

\subsubsection{The Classroom Teacher}

The classroom teacher was an American volunteer teacher. She had been teaching English in Vietnam for three years when the data collection was conducted. She had a 
master's degree in TESOL and had taught the class for a semester. She co-operated with the project but was not observed as part of the study. I talked to her about how the students would participate in the research. I explained to her that the students would be audio and video recorded as well being asked to write a journal and to have an interview with me. The teacher helped me by arranging the students in groups. This was part of the usual routine in the class. She did not revise her planned activities or methodology for the purposes of the research being conducted. This meant she was present in the classroom but was not involved in the groups. When the students were working in groups and had a question, they could raise their hands to ask her for information. Instances when the students asked for help from the teacher occurred very infrequently during the data collection period.

\subsubsection{The Roles of the Researcher}

During the data collection, I was a non-participant observer in the classroom. I performed the six common roles of a fieldwork researcher suggested by Stake (1995), with my adaptation: (1) Teacher role: to learn what the participants need in order to know how to participate in the research; (2) Advocate role: to discover the best arguments in justifying why certain procedures should be carried out; (3) Evaluator role: to give careful attention to the research's merits and shortcomings; (4) Biographer role: to describe in depth persons involved in the research; (5) Theorist role: to use sociocultural theory as the baseline to design the research and to interpret data; and (6) Interpreter role: to find new ways to make new findings comprehensible. 
I went to the natural environment of the classroom to observe the students. However, I was aware that I must never become so involved that I ceased to be an observer. I tried to avoid the "observer's paradox" (Labov, 1972) by not altering the normal daily routines of the classroom. When the students were working in groups, I stood quietly in a corner, operating the video camera. I handed the journals to the students during break time and collected the journals during break time the next day. I talked to the classroom teacher to make sure that all the classroom activities were organised as usual and the teacher did not revise the tasks for the research purpose. The students were randomly assigned to groups, and took part in the group activity as usual. They were not given any instructions beforehand to prepare them for the activity. Before each session, during break time, I informally told the students to be natural in doing what they usually did when they worked in groups. I told them that each of them would experience two kinds of group settings. I also aimed to adopt a detached and reflective approach as recommended by Hitchcock and Hughes (1989, p. 37) who pointed out, "Detachment and reflection are important skills for qualitative researchers to develop". I also conducted audio-recorded interviews with students out of the classroom context when each student had experienced working in both an unassisted and assisted group. I had the role of an "insider" and an "outsider". I was an "insider" from being a learner of English in Vietnam. I also had the advantage of having been a lecturer of English for seven years there. I know how the system works, the hierarchical structures of the community, the setting and the formal as well as informal behaviour systems. As a Vietnamese, I also understand the culture of Vietnam and the institutional and social context where I was investigating. I know Vietnamese ideology, traditions, customs, value systems and beliefs very well. My experience as an "insider" gave me insights into data interpretation. 
I was an "outsider" from living and studying abroad for a significant period of time: two years in the United States and three years in New Zealand. I had had time to reflect on the process of learning and teaching English in Vietnam and also on sociocultural theory. My extensive time abroad helped me to view afresh Vietnamese culture and its reflection in the classroom context.

\subsection{Classroom Physical Set-up}

Figure 2 shows the diagram of the classroom under observation in the current study. In this class, the teacher's desk was at the front and to the left of the students. The students sat closely together on shared benches set up in rows. The two groups to be observed sat near the door to avoid interfering with other students who were also working in groups but were not observed. The other groups operated as usual when the two groups under observation were recorded. When it was the turn for other students to be recorded, they moved into the shaded areas to work in an unassisted or assisted peer group. By the end of the data collection, every student had experienced the two kinds of group work. 


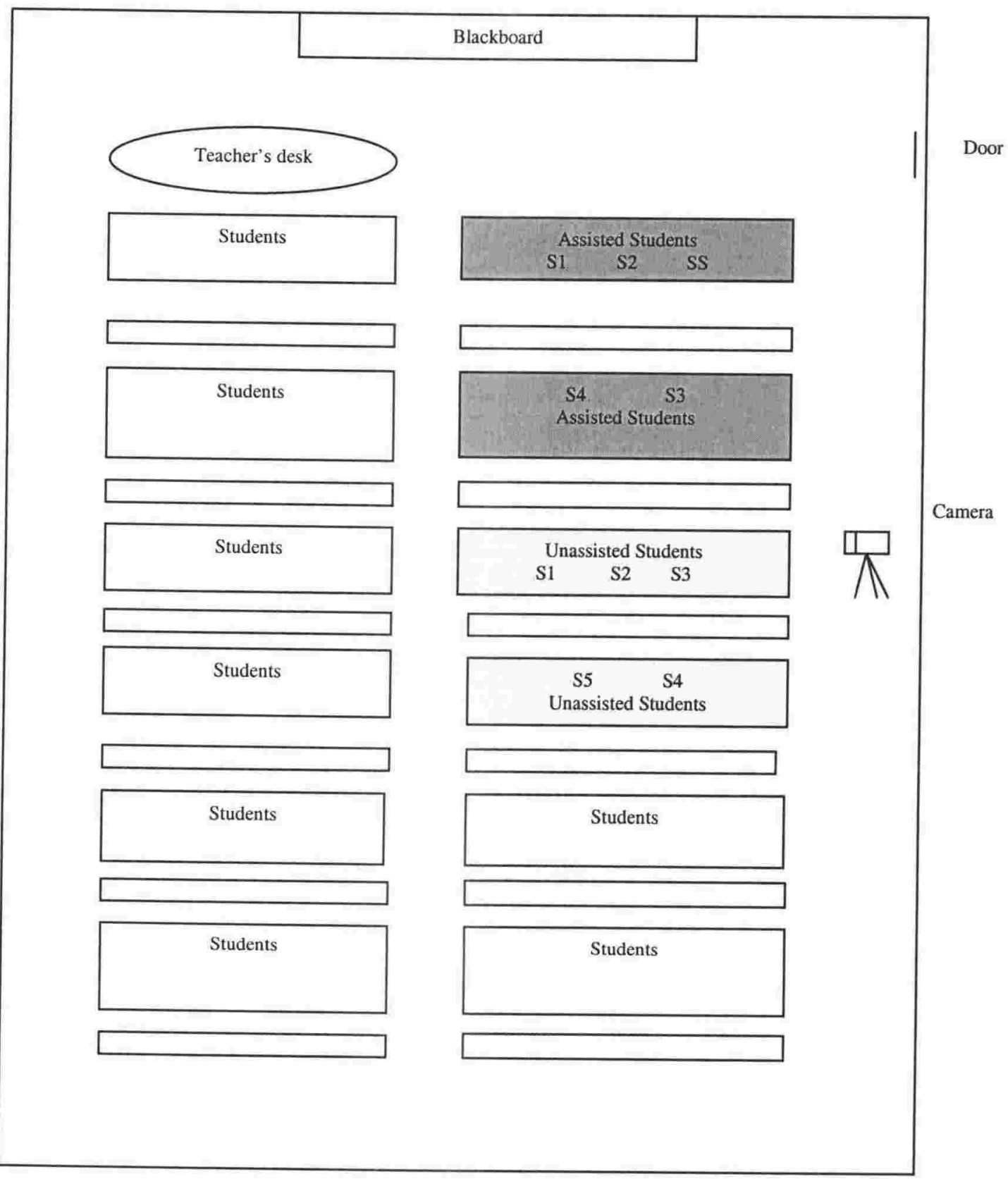

Figure 2: Diagram of the Classroom Setting and Data Collection 


\subsection{Data Collection}

\subsubsection{Audio Recordings}

Audio recording was used to capture students' natural speech in group work. All of the students wore the same kind of small button microphone attached with a clip to hook onto a button or shirt collar when working in the groups under observation. Two small Walkman cassette recorders were set up at the same time. Neither the microphones nor cassettes took up much space.

\subsubsection{Video Recordings}

"Observation directly records what people do, as distinct from what they say they do" (Denscombe, 1999 , p. 146). A portable camera was set up on a tripod in a corner as far from the students as the space of the classroom allowed. During the time allocated to group work, the camera focused alternately on the unassisted and assisted peer group for five minutes at a time. In the first few minutes of the first observation, the students seemed to be self-conscious. After the first observation, all appeared to be unaware of the camera. The video camera recorded how the students behaved and their use of materials. It also captured the gestures the students made during group interactions. The gestures however cannot be matched with the audio transcriptions because the video recording was restricted to intervals of five minutes for each group under observation. In addition, the students' speech could not be heard from the video recordings because the built-in microphone of the video camera failed to record clear speech produced by 
the students during group work. The video recordings only provided images of the upper parts of the students' bodies, their faces and their hands above the desks.

\subsubsection{Journals}

"Diaries, logs, and journals are important introspective tools in language research as they can provide a first person account of the experience of language learning" (Nunan, 1992, p. 118). After each group work session, each student was asked to write a journal entry. The journal was to collect the students' reflections on their experience of group work. Each student in the current study wrote at least two journal entries during the whole project. Some students wrote three entries because they had participated three times in the groups under observation to make the total of five students for each group for each observation. However, their third entries were not used for data analysis. To overcome the problem of lack of recall, guiding questions were provided in the journal (Appendix F and G) and the students completed an entry immediately after the group work.

There were eight journal questions for unassisted students. The journal for assisted students contained the same questions with two additional questions on the students' perception and evaluation of the role of the senior student. The journal questions were presented in both English and Vietnamese. The responses in Vietnamese were translated into English by the researcher. The first two questions focused on students' opportunities to speak in group work and were designed to help students recall experience in the group work. The next three questions examined the students' perception of the role of other peers in the group work and their experience of working 
in a group. The last three questions investigated affective factors and particularly whether the students enjoyed the group work or not.

The journals were given to the students after each session. The students took the journals home and returned them to the researcher the next day. By the end of the fieldwork, a total of ninety journal entries, written by the 45 students, were used for analysis.

\subsubsection{Interviews}

It was not assumed that all English learning necessarily occurred in the classroom, and a semi-structured interview was developed to investigate support for the students' learning of English outside the classroom. The semi-structured interviews were conducted immediately after each student had experienced both types of groups. The semi-structured interviews allowed me to control the dialogue because the nature of this data collection method is selective (Kelep-Malpo, 2003). Furthermore, Bell (1999, p. 138) pointed out, "The advantage of a focused interview is that an outline is established beforehand and so analysis is greatly simplified". In face-to-face interviews, I could question the students about their experience of being in the two kinds of groups. The semi-structured interview questions (Appendix I) were aimed at exploring how the participants compared the experience of being in unassisted and assisted peer groups. The journal provided students' reflections after a single group work experience whereas the interview could elicit a comparison in students' points of view of their two group work experiences. A particular benefit was that the face-to-face environment allowed for immediate clarification. 
Each student was interviewed for 20 minutes in Vietnamese. The interviews were taperecorded but not videotaped. Before the interviews, I had informed the participants about the tape recording, which was also explained in the consent form. At the beginning, I tried to establish rapport with the participants to make them feel at ease by having some friendly and informal talk. During the interviews, I took notes on important answers from the participants in relation to the interview questions in order to help with the transcription later when I returned to New Zealand.

None of those methods described above (audio-recording, video recording, journal writing, and interviews) could provide a complete description of the groups. Each method was designed to capture different kinds of data. The audio-recordings recorded the speech that the students produced during the group work. The videos revealed what the students did when there was a period of silence on the audio tapes, their gestures during group work, and their use of the artifacts. The journals recorded the students' reflections. Through the interviews, I asked for individual opinions and gathered more information in relation to what was written in the journals. The interviews also focused on aspects of the sociocultural context of Vietnam and institutional, social and personal factors. Both journals and interviews were based on the students' recall.

By the end of the data collection period of three months in 2002 , the following data had been collected for the main study:

Audio : 368 minutes of tapes (6 hours 8 minutes)

Video: 184 minutes of tapes (3 hours 4 minutes)

Journals: 90 entries

Interviews: 900 minutes of tapes (15 hours)

Artifacts: record of a textbook and other materials used during the group work. 


\subsection{Identification}

Each student was assigned an ID number. The journal entries and interview transcription of the same student were matched for analysis. SS, S1, S2, S3, S4 and S5 were used to refer to the senior student, and other students in turn in the audio transcriptions. That is, in each session, students were identified as S1, S2 etc. in the order they first spoke. The letter "U" refers to "Unassisted" group work experience and "A" refers to "Assisted" group work experience. The data from journal entries and interviews were coded in the following way,

$\begin{array}{ll}\text { Student ID-Unassisted-Journal } & \text { ID-U-J } \\ \text { Student ID-Assisted-Journal } & \text { ID-A-J } \\ \text { Student ID-Interview } & \text { ID-I }\end{array}$

The audio transcription was identified by a number for a sequence for analysis, whether the group was unassisted or assisted and the topic, for example, Sequence 01: Unassisted Group: Topic - Anger, and Sequence 02: Assisted Group: Topic - Animals.

\subsection{Analysis}

The coding, filing and categorising of the data were carried out in New Zealand when I had completed the fieldwork. The audio transcriptions were analysed first, then video records, journal entries, and finally interviews. I translated all speech and responses by the students in the audio transcriptions, journal entries and interviews in Vietnamese into English. The translated equivalents of the audio transcription are presented in square brackets [ ]. The Vietnamese responses in the journal entries and interviews are commented as translation from Vietnamese which is put in round brackets ( ). Some students used Vietnamese dialects which are different from region to region. The 
Vietnamese in the transcriptions has been reworded using standard Vietnamese. The diacritical marks of the Vietnamese words were omitted from the audio transcriptions due to the difficulties of using the software in English font. However, the meanings of Vietnamese words without diacritical marks are still able to be read by Vietnamese readers. Some students used Vietnamese or switched to combined Vietnamese and English in their journals. I also translated all these combined reports into English before analysing them. The data were categorised according to each research collection method, such as audio recording, video, journals and interviews. They were then stored in separate folders and compiled into areas relating to the research questions. They were viewed and re-viewed to enable the development of possible coding categories to emerge rather than analysed based on pre-set categories. During the process of synthesising data from the variety of sources, initial analysis and categorisation were revisited and revised. A table of the detailed coding scheme is included in Appendix J.

The coding starts with the initial of the data source, for example $\mathrm{T}$ for transcription, and continues with initials of the main categories or sub-categories, for example NTP for negotiation of task planning and procedures. The following sections discuss in detail the data analysis.

\subsubsection{Analysis of the Audio Transcriptions of Group Work}

A total of 360 minutes of tapes were used for data analysis. I transcribed the audio recordings with the help of a transcribing machine. An example of typed transcription of the audio recording of a group work session of both kinds of groups is included in Appendix K. Each group had one male only, so it was possible to identify the male 
student's voice. The senior was distinguished from other students as I clearly recognised her voice, and other students were identified because their VietnameseEnglish accents varied according to areas of origin and personal differences in voices. Sociocultural discourse analysis (see section 1.5, Chapter 1) was used to analyse the audio-taped transcription. According to Coll and Onrubia (1997), discourse is part of the joint activity in which participants are engaged in educational situations and its analysis reveals the contribution to the construction of shared meanings in the classroom. The names in the audio transcriptions are pseudonyms. The transcription was numbered according to the changes of speakers to count the number of utterances (see section 1.5, Chapter 1 for the definition of an utterance). This process was repeated for other topics. Utterances were numbered consecutively for each topic but not for the whole transcription of all the topics. The coding of the transcription then was carried out by segmenting each transcription of a group session into its constituent sequences and then each sequence was examined in the coding protocol corresponding to the categories in the coding instrument. The categories include the followings:

(1) Utterances: Students' participation in group work was examined in terms of the utterances they produced. For example, the following sequence has three utterances:

$01 \mathrm{~S} 1$ In a restaurant, the waiter ignores you and begins to serve other tables although you were sitting first.

02 S3 I will not come to that restaurant again.

$03 \mathrm{~S} 1$ But you need to eat.

(2) Negotiation: The categories for negotiation in this research include:

2.1) The negotiation of task planning and procedures: The process in which the students worked together on what they had to do to complete a language task.

2.2) The negotiation of understanding: This category was originally used by McDonald et al. (1991), who studied children just starting school who spent a 
lot of time working out what to do in class. McDonald et al. used three kinds of negotiated explanations to examine the interaction of students: negotiation of direct meaning, negotiation of understanding, and negotiation of the learning environment. The negotiation of direct meaning refers to understanding and comprehension of classroom tasks. The negotiation of understanding involves what can be called general or background understanding either about things in the classroom or the world in general. The negotiation of the learning environment means the understanding of the processes of a teaching-learning event. In the current study, the students had studied long enough to know the rules. The negotiation of understanding in the current research expresses interactive behaviours involving general or background understanding either about things in the classroom or the world in general. It is the process of shared understanding and knowledge of the topics under discussion. The negotiation of understanding in the current research also means negotiation of shared cultural meanings in tasks as designed by foreigners in relationship to Vietnamese cultural practices.

2.3) The negotiation of word meanings and forms: The students shared their knowledge of word meanings or forms by helping and explaining these to each other.

If an episode had different negotiations, all were examined.

(3) Laughter: Laughter episodes on the audiotapes were extracted to look for the reasons why the students laughed. 


\subsubsection{Videotape Analysis}

About 180 minutes of video tapes were viewed and reviewed. The episode of analysis was an interval of five minutes for each group as it was recorded. The interval was small enough to capture snapshots of starting points and ending points of each session of group work and to examine group dynamics, such as who was speaking, eye contact and gestures. The video tapes were also viewed to examine what the groups were doing when there were episodes of silence on the audio tapes.

\subsubsection{Journal Analysis}

Ninety journal entries written by the 45 students were used for analysis. The journals provided the students' reflections and perceptions of group work. The categories examined were:

(1) Opportunity to speak: The students reported their opportunities to speak in group work.

(2) Vocabulary: The students reported whether they learned vocabulary in group work. The subcategories included: new words, joint learning of vocabulary, collective memory of vocabulary, and unsuccessful vocabulary learning.

(3) Discussion of a topic: How an assigned topic was discussed in a group. The subcategories include: students' knowledge and evaluation of the topics, and whether the discussion was effective.

(4) Students' feelings: Enjoyment, stress and other feelings as reported by the students. 
(5) Students' evaluation and perception of the senior student's role: How the senior student helped with the group work.

\subsubsection{Interview Analysis}

I transcribed all of the 45 interviews with the aid of a transcribing machine, and translated all the Vietnamese responses into English. The interview transcriptions were taken from approximately 18 hours of total recorded time on cassette tape. Phonological and paralinguistic information was omitted; the focus was on content. A sample of interview transcription is provided in Appendix L. The interviews were examined on the basis of the semi-structured and predetermined questions used as guides. The categories include:

(1) Learning vocabulary at home: how the students learned vocabulary at home.

(2) Artifacts: what materials and learning tools the students had access to outside the classroom; what artifacts the students used for study in class.

(3) Students' perception and evaluation of speaking English with other students.

(4) Students' perception and evaluation of speaking English with a senior student, for example, whether they liked to speak with a more advanced student and why or why not.

(5) Students' perception and evaluation of group work, for example, if they would like to have more group work and why.

(6) Learning vocabulary in the group work under observation.

(7) Topic discussion in the group work under observation. 
(8) Students' feelings: positive and negative emotions as a result of participation in the group work under observation.

(9) Learning English outside the classroom: with foreigners, friends, family members, and the students' evaluation of the importance of speaking English outside the classroom.

(10) Reasons for learning English: social and individual factors.

\subsubsection{Artifact Analysis}

The use of the textbook, handouts and other materials was examined. Aspects that were taken into account include:

(1) The textbook and classroom handouts: topics assigned, task requirement as set out in the textbook and goals and objectives of the textbook.

(2) Curriculum book: goals and objectives of the class under observation.

(3) Other artifacts: materials that the students used in the process of group work, for example, classroom handouts.

\subsection{Research Validity}

As discussed in section 4.3, this is a qualitative study; therefore, generalisability may not be a relevant standard with which to evaluate its worth. The research did not attempt to generalise the findings but sought to obtain insights into the group work processes of two different settings which were analysed through a sociocultural lens. The research validity was strengthened in a number of ways. First, the current research employed methodological triangulation to collect a chain of evidence in data collection. 
The rationale for methodological triangulation is that the flaws of one method are often strengths of another, and by combining methods, observers can achieve the best of each, while overcoming their unique deficiencies (Denzin, 1970). In this study, audio recording, video recording, journal writing, interviews, and artifact examination were used. Each of them gave different sources of inter-personal and intra-personal information pertaining to the research questions. No single method, of all listed, could elicit the complete data, as a result, a combination was used. The data collection methods captured what participants said (audiotapes), what participants wrote (journals), what participants thought (interviews) and how participants used artifacts and produced gestures that could not captured by the audio transcriptions (videos). The data gathered through these triangulated means were then cross-analysed in order to obtain an in-depth understanding of group work processes and of the meanings underlying them. According to Anderson (1998, p. 159) triangulation in interpreting findings helps to test alternative ideas, identify negative cases and point the analysis towards a clear conclusion based on the evidence collected.

Second, "thick description" (Geertz, 1993) was employed in the research. Thick description means to supply a substantial amount of clear and detailed information about the issue studied and the setting in which that issue was investigated. The current study described a holistic picture of the participant students in their social, political, cultural, and institutional context. The thick description of the research participants, settings, research instruments, research design and the findings of the current research may be transferred to other similar contexts using group work for teaching and learning English as a foreign language. Firestone (1993) and Mayan (2001) considered "thick description" can help transfer the research findings to contexts outside the study 
situation to other settings. Recommendations from this study for other Vietnamese institutions of English and foreign language institutions elsewhere may be beneficial to those in similar situations.

Third, as mentioned in section 4.8 .4 in this chapter, I have the insights of both an "outsider" and an "insider" in interpreting the data. The analysis and interpretation of data specific in the framework of sociocultural theory used in the current research thus, to some extent, has content validity.

Lastly, the research validity was also strengthened in the category of data "trustworthiness" (Mishler, 2000). Data trustworthiness can establish confidence in the findings (Lincoln \& Guba, 1985). To search for trustworthiness, I did not "distort" participants' meanings (Brown, 1989, p. 280), and my conclusions or claims had supportive documentation. In this research, for each point discussed, different sources of data are quoted and illustrated.

\subsection{Research Assumptions}

The research was based on three methodological assumptions. Firstly, qualitative methods were suitable to investigate the processes which occurred when groups of students were engaging in practising English as a foreign language. Secondly, students were likely to be honest in their responses to interviews and in their journal entries. Finally, if students answered in Vietnamese, it was possible to provide a translation for Vietnamese utterances and responses that was true to the meanings the students wanted to express. In addition, the current study assumed that the differences in the starting 
levels of the participants students are considered irrelevant, and the content of the journal is seen as a function of the students' cognitive and linguistic abilities.

\subsection{Limitations}

This is an in-depth qualitative study in one classroom in one educational institution with some extension to the broader culture and society where the participants lived. The data collected were from the students' speech when they were working in groups and from their perceptions of their own learning as written in their journal entries and reported through the interviews with the researcher. The study did not investigate independent learning as a result of participating in group work in an EFL classroom. In investigating demonstrated gains in learning English, the study focused on vocabulary acquisition only.

The research was carried out in a natural classroom with an awareness of the need not to interfere with the normal classroom processes. The data collection depended on the students' presence in the class. A student who participated in the unassisted peer group was not necessarily present the next day and therefore available for experience in the assisted peer group. In addition, the groups differed in size with regard to the junior students. There were five junior students in the unassisted peer groups and four junior students and a senior student in the assisted groups. The data from the videos could not be matched with the audio-transcriptions. However, the videos could be used to examine the gestures the students made and how they used the artifacts. Snapshots at the beginning and ending of the discussions were viewed for the group work processes at these points and the students' eye contact patterns. Also due to the small percentage 
of volunteer male students ( 8 male students as opposed to 37 female students), the study could not explore gender differences in group work.

The students in Vietnam sit on fixed benches. As the classroom diagram shows (see

Figure 2), the students could not move in a circle when they worked in groups. The camera could not focus on all 10 students at the same time. Instead, I video-taped each group for five minutes. The records of nonverbal communication as collected from the videotapes were thus treated as exploratory.

\subsection{Summary}

This chapter presented the research design. There were two different group settings in the current study: unassisted peer groups, each of five peers from the same class, and assisted peer groups, each of four peers from the same class and a senior student from a more advanced class. The introduction of the senior student as a new member in the group aimed to capture differences of the roles of peers and group processes in the two different settings. Different data collection methods, namely, audio recordings, video recordings, journal writing, interviews were used. The chapter provided the "thick description" of the research participants and the context in which the data were collected. It also provided a complete description of categories, coding scheme and data analysis procedures. The research validity was strengthened by different strategies including triangulation in data collection and interpretation, thick description and establishing data trustworthiness. 


\section{Chapter 5}

\section{GROUP DISCUSSION OF TOPICS}

\subsection{Introduction}

This chapter discusses how students in each type of group carried out the group discussions. It addresses the first research sub-question: "What are the differences in the way a topic is discussed?" The students were required to discuss a number of topics or themes set out in the textbook. The chapter begins with an overview of what the requirements for each discussion were and to what extent the students completed the requirements. Then it focuses on the topics and the discussions. The topics were considered with regard to whether they were interesting to the students, whether the students gained knowledge from them and how they used their prior knowledge in the discussions. They were then examined according to: whether the students considered a discussion to be effective or not. Non-verbal communication, mainly eye contact and gestures, are reported. The data came from the audio transcriptions, video records, journal entries, and interviews. Learning is defined as occurring

When an individual comes to know something he [sic] did not know before or becomes able to do something he could not do before. (Ingram, 1975, p. 218)

The chapter ends with a discussion of what the students said that they had learned.

\subsection{Topics}

In this section, I provide a brief overview of the requirements for in-class discussion of each topic and how the students in the two kinds of groups carried out the discussions. 
Topic 1: Anger. The students had to discuss 10 situations regarding how they would react to an incident which might make them feel angry. For example, "What would you do if a visitor breaks your favourite vase?" The unassisted group discussed all ten situations. They produced two Vietnamese utterances. The assisted group completed only six out of ten situations but for each situation all the members of the group contributed ideas. The senior student also called for group agreement or disagreement with each statement.

Topic 2: Animals. The students had to discuss twelve animals according to whether they are eaten, kept as pets, treated as wild, or used as a working animal. The textbook provided a list of the twelve animals. The assisted group talked about nine animals, two items more than the unassisted group. When deciding what the animals should be used for, the students in both groups explained why and how the animals were used in certain ways. Neither of the groups completed the discussion as required. The students in both groups used only English in discussion.

Topic 3: Vacation. The students were to talk about where to take a vacation, when and what to do. The classroom teacher however modified the textbook requirement by asking the students to choose three places (cities or countries), three restaurants and three leisure activities. When the unassisted group was not clear about the required task, they used Vietnamese to figure it out. There was confusion both at the beginning and in the middle of the discussion. The group did not develop a clear focus. In the assisted group, the senior student provided a clear introduction. She explained the required tasks. The assisted students understood the task and proceeded without 
confusion. The senior student kept them focused on the task and also added more specific contexts for the discussion.

Topic 4: Good luck and bad luck. The students were asked to think of the things in their country, which bring good or bad luck. Approximately half of the total utterances of the unassisted students were in Vietnamese. The students used Vietnamese to plan the task and to figure out what they had to do. In the assisted group, the senior student rephrased the required task for the students in her group by saying, "So we are talking about good luck and bad luck. Can you tell me what is considered good luck and bad luck in Vietnam?" When one student presented an idea, the senior student called for agreement or disagreement from other students so that each idea was discussed in depth. The assisted group discussed one item fewer than the unassisted group (18 as opposed to 19) but they tended to discuss each in greater depth.

Session 5: Review. This was a review session. The students were to ask two questions for each topic that they had studied. The unassisted students did not understand clearly what they had to do. In the first four minutes, they spoke in Vietnamese about the planning and procedures of the task. Forty-three out of 102 utterances of the whole discussion were in Vietnamese. The unassisted group prepared 14 questions and ended the discussion abruptly. In the assisted group, the senior student started the discussion. She stated clearly from the beginning what the students had to do and encouraged the students to speak in English. The students prepared 20 questions.

Topic 6: Rural and urban. The students were to discuss the differences and similarities between the city and the country. The textbook provided a list of categories but the 
classroom teacher asked the students to talk about only four of these. The unassisted students thought they had to discuss all of them. They experienced confusion (see sequences 02,04 , and 06 in Chapter 7) and did not know exactly what they had to do. In the assisted group, the senior student started the discussion. She repeated clearly the instruction from the classroom teacher. For each category to be discussed, all students contributed ideas. The senior student also called for evaluations by asking group members, "Do you think so? Do you agree or disagree?" The students did not use Vietnamese in this session.

Topic 7: Traditional and modern. The students were given pictures of rooms by the classroom teacher. They had to imagine who might live in each room. The textbook provided some questions, "What kind of people are they? How old are they? What jobs do they do? Have they got any children? What sort of clothes do they wear? What is their taste in music? What about their taste in food? What does the rest of the house look like?" The unassisted students discussed three pictures. Their discussion was sometimes off the assigned topic. The assisted students discussed four pictures and kept to the topic. The senior student started and ended the discussion.

Topic 8: Advertising. The students had to create an advertisement to sell a product. There was no negotiation about the choice of the product in the unassisted group. One student wanted to advertise a pen but the ensuing discussion was not about creating an advertisement. The student who wanted to advertise a pen ( $\mathrm{S} 2$ in this case) tried to persuade other students to buy the pen. The unassisted students occasionally used Vietnamese. In the assisted group, the students first chose a product. The group then discussed ways to advertise it. The senior student started the talk. The first three 
minutes were used for one particular product choice and then they changed to another product. The senior student suggested changing the product to be advertised and the students followed her suggestion.

Topic 9: Becoming an adult. The students had to decide at what age people in Britain and in Vietnam could do certain things. The textbook provided a list of "landmarks" when young people in Britain are allowed to do certain things and a chart of a range of ages to match with the landmarks. The unassisted group discussed the whole list. They produced four Vietnamese utterances and some utterances which were off the topic. The students did not check the answers as required by the classroom teacher. In the assisted group, the senior student started the discussion and assisted the students in task procedures and planning. The students followed the procedures outlined in the book. The senior student told the students when to start on a new statement in the text. Then they checked the answers in the textbook as required.

Topic 10: Working styles. The students had to decide whether each statement in a list of twelve in the textbook would be likely to be made about Japanese or European companies. The students in the unassisted group presented their ideas on each statement but the ending came abruptly because they ran out of time. In the assisted group, the senior student started the conversation and explained to the group what they had to do. There was a clear beginning, ending and turning point for discussion of each statement in the textbook. The discussion of the assisted group was structured.

Topic 11: Work and salary. The students had to mark on a chart the position of eleven jobs listed in the textbook regarding their salary and prestige. In the unassisted group, 
there was no clear signal as to where the task began and ended. The unassisted students frequently used Vietnamese. In the assisted group, the senior student started the conversation and explained to the students what they had to do. The assisted group used a chart to mark the position, the salary and prestige of all the jobs provided. For example, a teacher was ranked with the highest prestige but low to medium salary. A police officer had less prestige than a teacher but a higher salary. A nurse had medium prestige and salary. The senior student helped the students with the task procedures and kept the group on task. Both groups ran out of time to complete the discussion.

Topic 12: Taking risks. The students had to answer questions about taking risks. The textbook provided a list of questions, for example, "Would you make a parachute jump?" The textbook also provided a number score for the answers of the questions. A "yes" answer to a question is counted with five points, a "maybe" answer three points, and "no" answer one point. The students had to count up the total score for all the answers to see how willing each person in the group was to take risks. In the unassisted group, the students answered seven questions out of the ten required. They did not count up the total score for each person to see who was the most willing to take risks. The students went off task when they talked about the risk of buying shares. The assisted students completed the task and calculated the final score to see who in the group was the most willing to take risks. For example, one student (S4), the bravest, had the score of 27. Another student, S3, had a score of 15. Both S1 and S2 had a score of 12 . The senior student explained to the students what they had to do, and kept them on task. 
The audio transcriptions showed that while the unassisted students were often confused about the task requirements during four group sessions, the assisted students were not. Three-quarters of all unassisted group tasks (9 out of 12) were not completed. In the assisted group one quarter ( 3 out of 12) of all the tasks were not completed. The senior student always started the discussion, and she helped the students understand clearly what they had to do and kept them focused. The processes of interaction in the two kinds of groups will be presented in Chapters 6 and 7. The following section reports the data from the journal entries with regard to the discussions and topics in each type of group.

\subsection{Journals}

The journal questions (Appendices F and G) did not directly ask the students about the discussions of the topics but about group work in general. As discussion was the requirement of the group sessions, the students reported their experience and provided an evaluation of whether the discussion was satisfactory or not.

\subsubsection{Unassisted Group Discussions}

The reports from the students in the unassisted groups showed that only one unassisted student considered the group had a satisfactory discussion, "We had group collaboration by giving advice to complete the task" (37-U-J-ED). Nine unassisted students wrote that the discussions in their groups were not satisfactory due to reasons such as: the group did not discuss the topic deeply enough (two responses), the students did not understand clearly what they had to do (two responses), time constraints (two 
responses), limited proficiency of English (one response), familiar topic (one response), and insufficient preparation for the topic (one response). Their comments were, for example:

I did not understand the topic at first. The discussion was simple and repeated. At the end of the discussion, there was not a concrete conclusion. (5-U-J-ID)

The discussion was not fluent and slow because of different level of competency and the fact that each person had to give out his/her own ideas. (20-U-J-ID)

Although the topic was interesting, the discussion was not deep. Because a member chose to advertise a pen the task was not interesting. We did not discuss the topic enough and exactly. (36-U-J-ID)

The comments above provide some explanations of the students for their evaluation of the efficiency of the unassisted group discussions. The discussions were reported as unsatisfactory work due to a variety of factors.

\subsubsection{Assisted Group Discussions}

More assisted students wrote about the group discussions in the journal entries than the students in the unassisted groups did. Seven students considered that the assisted group discussions were effective. Some reports follow,

There was collaboration among the group so that each member could be helped with new ideas. (03-A-J-ED)

The senior student led the discussion and called for collaboration from others. (28-A-J-ED)

It was a review session so I found it difficult to ask interesting questions. But with assistance of the senior student, we could think of many questions for each topic in a short time. (18-A-J-ED)

In the assisted students' view, the discussions were effective because there was help from other group members (two responses), and the senior student's suggestions and 
help with the discussion (two responses). According to some students in the assisted groups, the discussions were effective because they were expanded and focused (two responses), and there were ideas from several students (one response). However, four assisted students reported that discussions were unsuccessful. The ineffective discussion was due to time constraints (three responses) and limited English (one response).

We did not have enough time because we discussed too profoundly. (12-A-J-ID)

Because of our limited English, we could not express fully our points of view. (32-A-J-ID)

We did not have enough time to make an advertisement. (30-A-J-ID)

Whether in an unassisted or assisted group, the students' perception of the success of the discussion was similar. That is, successful discussion depended on the collaboration of group members, whereas different reasons were given for ineffective discussions. The students in the unassisted groups reported unclear understanding of task requirements, time constraints and limited English proficiency. The assisted students mainly reported the time constraints.

\subsubsection{Knowledge of the Topics}

In the journals, I asked the students, "Did the discussion today bring you knowledge of English and other things? If yes, what was it? If no, please explain?" The questions aimed to encourage the students to write about any of the things that they had learned from the discussions whether this was aspects of the English language or knowledge of the topics. The Vietnamese form of the questions in the journals gave the students a clearer idea of what to write. The students understood the questions and wrote about 
their experience. In their journals, the students wrote about the topics, focusing on the extent of their understanding of these.

Twenty-two unassisted and 19 assisted students reported that participating in the discussion helped them know more about the topics and expand their knowledge. However, three unassisted students claimed that the discussion did not bring them any knowledge. None of the assisted students reported that the discussion did not bring them knowledge. Some comments follow:

New knowledge of the country of Holland, its people, and natural conditions. (13-U-TK)

The discussion brought me knowledge of good luck and bad luck in my country. (18-U-TK)

I knew more about the difference between life in the city and life in the countryside in many aspects. (29-U-TK)

I knew about some places to visit in many countries and in my country. I learned more things from my friends' ideas. (04-A-J-TK)

Knowledge of different jobs in society. For example, teaching in Vietnam gets average salary but high prestige, which is different in Britain. (45-A-J-TK)

I learnt about classical music and old and modern rooms. (34-A-J-TK)

The knowledge reported was strongly associated with the types of topics under discussion. The students' knowledge, according to these reports, seemed to be extended through interaction with the other members in the groups. For example, the textbook did not provide information about Vietnamese belief in good luck and bad luck or the differences between the countryside and the city. It only asked the students to talk about the topics. Student 04 in the examples above mentioned knowledge that came from the discussion of the topics and also from the contributions of group members. In this way, the topics and the task requirements triggered talk and shared understanding 
among the students. They reported that the discussions brought them knowledge when they listened to group members and the ideas they contributed. However, the textbook appeared to be important in bringing the students new understanding of the topics under discussion (see Chapter 9 for a discussion of the roles of the textbook and other artifacts).

The three unassisted students who reported not gaining any knowledge gave the reasons as follows,

The discussion did not bring me new knowledge today because we talked about the topic that was very familiar to me. (07-U-J-TK)

The topic today was about laws and we do not know much about it. The topic limited our discussion today. (41-U-J-TK)

The topic was about laws in Britain and we do not know much about them so we discussed based on our guess and we did not know if our guess was correct. (42-U-J-TK)

If extending topic knowledge base is part of the aim, then the reports above suggest that knowledge introduced in the discussion must be something new to the students but not too foreign. If the students were familiar with the topics or knew something about them, they did not consider the discussions brought them knowledge (for example, students 07 and 41). Student 42, however, considered knowledge came from prior knowledge and then from sharing with other members. If this student did not know about the topic, it would be impossible or very difficult to bring about further knowledge through discussion. The students' reports also suggested that knowledge came from their existing cultural and social backgrounds, and their familiarity with the topics. Student 42 mentioned the need for students to know "if our guess was correct". 
The reports in the journal entries revealed that the students, when working in groups, were likely to obtain knowledge of the topics under discussion by talking and listening to ideas from each other. However, it is possible that some students might not be listening or were pretending to listen. Although the main aim of the task was not to acquire knowledge but to practise a foreign language, the students reported incidental learning of new facts. The knowledge could be their understanding of the topics and the world in general. The students used English and also Vietnamese (see Chapter 7) as a means of exchanging and sharing the facts and ideas with group members. Students' prior knowledge of English and prior knowledge of the topics in their first language were used in the discussions.

\subsubsection{Students' Evaluation of the Topics}

In the journal entries, the students also reported whether the topics under discussion were interesting to them or not. Nine unassisted students wrote about the topics but only five of those considered the topics were interesting and the other four considered the topics were not interesting. Six assisted students wrote about the topics but only three of them considered the topics were interesting. Examples follow:

Yes, talking about health and people in the country and the city was the most interesting. The topic was easy to discuss. (27-U-J-TI)

Yes, I liked to talk about the people who lived in the house. (33-U-J-TI)

I think anger was an interesting topic for discussion. (09-A-J-TI)

I like the topic becoming an adult. (36-A-J-TI)

Some students reported that the topics were not interesting. Here are some examples:

The topic [good luck and bad luck] is not very interesting because I am not a superstitious person. (19-U-J-TI) 
It seemed to be an unfamiliar topic, we had never thought of a journey or a vacation in other countries. (14-U-J-TI)

The disadvantage was that we had studied all these topics so I found it difficult to make interesting questions. [This was a review session] (18-A-J-TI)

The topic for discussion today [working styles] was not very interesting and rather difficult. (40-A-J-TI)

The statements above show that the students' interest in the topics depended on the students' understanding and familiarity with the topics, and whether the topics were relevant to Vietnamese culture. Some unassisted students enjoyed the topics, such as animals, good luck and bad luck, rural and urban, and traditional and modern. Some assisted students liked the topics, such as anger, advertisement, and becoming an adult. Some unassisted students did not like the topics: animals, advertising, vacation, and good luck and bad luck whereas some assisted students reported that they did not find the topic working styles and the review session to be interesting to discuss.

In short, the extent to which students liked the topics varied in the two group settings. Students' interest in the topics was based on personal choice, their prior knowledge of the topics, familiarity with the topics, and English competence. The interest differed in the two kinds of groups. The students' reports in the journals showed that that they did not like the topics working styles and vacation because these topics were not familiar to them. The topic working style asked the students to talk about working styles of Japanese and European people, which were also foreign to the students. Vietnamese people rarely travel. Therefore, the students probably found it difficult to talk about the vacation as required. However, their evaluation of the discussions could be due to other factors, such as the requirements of the discussion as set out in the textbook, the 
classroom atmosphere, and affective factors at the time the students were discussing the topics.

\subsection{Interviews}

The interview was held after a student had experienced both the unassisted and assisted group sessions. In the interviews, I asked the students: "In what ways did your experience in the unassisted group and the assisted group differ?" Their responses, translated from Vietnamese, revealed the student's evaluation of the discussions in the two group settings.

\subsubsection{Unassisted Peer Groups}

Three unassisted students stated that they were freer to speak in the unassisted groups, and two reported they felt more cheerful and comfortable:

We were not task-focused but we were comfortable and not afraid of making mistakes. (35-I)

Without the senior student, we just listened and did not correct each other. We were free to speak what we wanted to say. (43-I)

Without the senior student, we did not follow any order, we did not discuss the topic in depth but we were more natural and spoke more. We were cheerful and when we could not express ourselves, we spoke Vietnamese. (24-I)

The absence of the senior student was one of the factors that led to the evaluation of unsuccessful/unsatisfactory group discussions among these unassisted students. There was not an authority or a "teacher" figure in the group to control or tell the group what to do. The senior student, to some extent, was a stranger because she was from another class. The students in the current study had known each other and studied in the same 
class for some period of time. However, most of the students evaluating the unassisted group work made more negative than positive comments. The unassisted students in the interviews reported the experience of unsuccessful group discussion because the discussion was incomplete (eight responses), boring (seven responses), unequal in idea contribution (seven responses), not focused (six responses), and unclear about task planning (six responses). Following are some reports:

We answered the questions from the book but we did not discuss them. It was less interesting than the assisted groups because everyone paid attention only to his part. (04-I)

Without the senior student, we did not understand clearly what we had to do. We spoke off the topic and did not discuss the topic in depth. There were no suggestions. We were confused and spoke with difficulty. (11-I)

We did not understand clearly what we had to say. Whoever wanted to speak contributed ideas, if not he/she kept silent. The group did not pay attention to slow members. Two students in the group did not speak much. (12-I)

\subsubsection{Assisted Peer Groups}

Nineteen students in the assisted groups reported that the senior student's suggestions helped them with the discussions. These reports corroborated the data from the audio transcriptions. The transcriptions showed that the senior student always started the discussions. During a discussion, she prompted the students based on the textbook questions. She also explained new words. More details about the role of the senior student and the processes of group work are presented in Chapters 7 and 8 .

Sixteen students considered the assisted group discussions to be more interesting and exciting than the unassisted group discussions. The reasons included that: there was 
collective contribution of ideas (20 responses), faster completion of task (eight responses), and expanded discussion (eight responses). Some examples follow,

With the senior student, we discussed. I thought more deeply because the senior student asked me more questions. Other students contributed ideas. I felt more motivated when I spoke in the group with a senior student. (04-I)

The group was more exciting and motivating. With the senior student, I had more ideas. I had to think more about my opinions because I had to speak to a senior student. With friends, we were at the same level, I was more natural. (03I)

The senior student helped to direct the discussion, guided us, and asked for more opinions. With the senior student, the group work was more exciting and effective. The senior student helped me to express ideas. (09-I)

With the senior student, we were focused. We had more ideas. She suggested ideas to us and posed a lot of questions. The discussion was clearer and more fluent. The process was faster. (20-I)

She helped to start the discussion and gave suggestions so that we could express more ideas. (30-I)

According to the students' responses, the unassisted groups did not discuss the topics as successfully and efficiently as the assisted groups. The reasons were that there was not a starter, moderator, and facilitator in the groups. The assisted groups had the help of the senior student who knew how to start the discussion as well as calling for the contribution of ideas from all the members. She also gave suggestions so that the groups completed the tasks and expanded the discussions.

The reports from the students also suggested that emotional factors cannot be separated from interaction in group work. The discussion made them feel cheerful, free, comfortable, or that they had achieved something. It also caused the feelings of boredom, or lack of achievement. More details on students' feelings will be reported in Chapter 10. 


\subsection{Group Work with Peers or With Peers and the More Knowledgeable Peer}

In the journals, when asked "Was the senior student helpful to you today? If yes, please explain and give examples" and "What do you think were the disadvantages of having the senior student in the group work today? Please explain and give examples", all 45 students answered that the senior peer was helpful. In general, the students reported that the senior student helped them to start the discussions. In addition, she encouraged the group members to speak by giving explanations and suggestions. She helped with vocabulary when the students could not find words to express themselves. She provided correction for linguistic mistakes. Speaking with the senior student was reported to help improve speaking skills. However, eight students reported some disadvantages of working in the groups with the senior student. Four of these students pointed out that the senior student spoke too fast and they found it difficult to follow her. Two others reported the loss of independence and freedom working with the senior student. Another two reported they felt less confident in speaking to her.

In the interviews, I asked the students, "Do you think it is a good idea to learn from other students? Please explain if yes or no." Twenty-one students reported that they liked to learn with other students in groups because they had opportunities to learn vocabulary from friends. Group work was an opportunity to exchange and share ideas among friends (18 responses). Overall the students appreciated working in groups with classmates because they benefited from the other students' understanding of how to learn English (eight responses), linguistic correction (six responses), explanation (six responses), and recommendation of materials (two responses). Working with other group members made the students feel motivated (three responses), comfortable or 
natural (two responses), confident (seven responses), and know more about their friends (two responses). The students also thought that group work with peers could help them improve their speaking skills, grammar, and sentence structures. One student reported that friends in group work sometimes used Vietnamese and this made her/him feel at ease. Interestingly, the students used the words "bạn" or "friend" to address group members or classmates in writing their journals.

Generally, the students liked working in groups whether unassisted or assisted. Fortyone out of 45 students, when interviewed, reported that they liked working in groups with classmates. Two of them said that they liked working with classmates in groups but with active and more capable members only.

An interesting category was the two students who thought that classmates did not help because they competed for scholarships or had the same level of English. In Vietnam, students are given scholarships based on their achievement in the class. This could have been a factor making for competitiveness.

\subsection{Video Recordings}

This section reports the forms of nonverbal communication as recorded on the video tapes. The non-verbal communication included eye contact and gestures which entail an ability to coordinate one's actions with others. The camera focused on each group, unassisted and assisted, for five minutes before moving to the other group. It captured only part of what was going on during group discussions. Therefore, snapshots of the first two students to speak in a discussion and snapshots at the end of a discussion were 
chosen for analysis. These snapshots showed group processes at the starting and ending of the 12 group sessions in each type of group setting.

\subsubsection{Starting Snapshots}

All the starting snapshots of the unassisted and assisted group sessions were viewed and reviewed. The snapshots of the unassisted groups showed that an apparently assertive student started the discussion and might look at any student in the group. The first person who replied had eye contact with the first speaker, or any student in the group, or looked at the textbook. In the assisted groups, the senior student started the discussion. She spoke while other students were looking at the textbook. She was always the first person to speak. She looked either at a particular student or all the students in the group. The first person to reply looked at the senior student.

At the beginning of the discussion, the unassisted groups did not have a clearly defined leadership position. In the assisted groups, the senior student directed the students to look at the textbook for information to discuss. The eye contact in the unassisted groups was in an irregular pattern whereas in the assisted groups, the senior student had eye contact with other group members and the person who spoke second usually looked at the senior student. The eye contact pattern suggests differences in power relationship in the two groups. The unassisted groups were freer and the assisted groups attended to what being said by looking at the senior student. Alternatively, the assisted students could have been looking at the senior student to show their respect for her. 


\subsubsection{Ending Snapshots}

All the ending snapshots of the unassisted and assisted group work were viewed and reviewed. The pattern of eye contact among the unassisted students varied. The last student to speak in the unassisted groups might look at any student in the group. In two instances, all members looked at the speaker in the group. In two snapshots, there was eye contact between the last student to speak and another student in the group. In one instance, there was eye contact between the last student to speak with all other members, and in another, the last student looked at the textbook and spoke.

In the assisted groups, the ending snapshots showed that individuals or students in groups looked at the senior student when they presented ideas or when they listened to her speak. The eye contact pattern was generally dyadic between the senior student and one student in the group or between two students. It was sometimes between the senior student and two other students in the group. Also the senior student sometimes received eye contact from all students in the group. The eye contact pattern in the assisted groups varied slightly but the senior student was the central focus.

The different composition of groups created different group interactions at the starting and ending points. The unassisted groups were less predictable in the pattern of eye contact at the beginning and ending of the discussions. In the assisted groups, the senior student was always the first person to speak. As a result, the first person to reply to the senior student had eye contact with the senior student. 


\subsubsection{Gestures}

I viewed all the video recordings and wrote down all descriptions of physical movements and gestures during group interaction to examine specific patterns. For a definition of gesture, see section 3.3.2 in Chapter 3. In the current study, gestures included movement of a hand, the head, and or the body when the students were talking to each other in groups. The number and types of gestures were then tallied. The videos showed the differences in the use of gestures in communication in the two kinds of groups in 12 group sessions:

Table 1: Number and Types of Gestures Used by Unassisted and Assisted Students

\begin{tabular}{lll}
\hline \multirow{2}{*}{ Types of gestures } & Unassisted students $(\mathrm{N}=60)$ & Assisted students $(\mathrm{N}=48)$ \\
\cline { 2 - 3 } & Number & Number \\
\hline Moving hands & 12 & 6 \\
Pointing & 11 & 1 \\
Nodding & 5 & 10 \\
Others & 2 & 2 \\
\hline Total & 30 & 19 \\
Note: Pointing at objects includes pointing at the textbook, the blackboard, the handout, \\
pictures, and a pen. The number of the unassisted students was counted by 5 students in a group \\
in 12 sessions and by 4 students in 12 sessions in the assisted groups. The senior student's \\
gestures are not tallied in Table 1.
\end{tabular}

The students in the unassisted peer groups moved their hands more often than making any other types of gestures. Usually the students in any kind of the groups moved one hand up and down or in a broken circle, and in and out toward the body. They moved a hand to illustrate or explain points when they were speaking. For example, in the topic anger, an assisted male student could not express himself completely and he moved his hand around in a circular fashion. The senior student broke in to help the student complete his sentence. In the topic animals, an unassisted student was speaking and moving her hand up and down. The group then laughed. It could have been the student's speech, or her gesture, that made the group laugh. 
A gesture which appeared frequently in the videos is nodding, five instances in the unassisted groups and 10 in the assisted groups. Nodding in the assisted groups happened more often because when the senior student was speaking other members who were listening to her nodded their heads. These gestures indicate that the students showed their agreement with the senior student's statement, or they probably wanted to say "yes". The audio transcriptions showed that the senior student asked a lot of questions, which led to some "yes", or "agreement" nodding from the assisted groups. The unassisted students also nodded as a reply to the previous speakers. As in some other cultures, the nodding and shaking of heads in Vietnam means "yes" or "no" or expressions of agreement or disagreement.

The unassisted students pointed more often to the textbook and other artifacts, such as the blackboard, pictures, and a pen. The videos showed that in the assisted groups, the senior student usually pointed to the textbook for the students to locate the information. As a result, the students in the assisted groups pointed to artifacts only once as opposed to 11 times in the unassisted groups. Pointing denotes the meaning of attracting the attention of the other members, and also of showing a source of information. The unassisted students pointed at the pictures when they were discussing the photos of traditional and modern houses, which the classroom teacher gave them. An example of pointing at a pen happened when the unassisted students were asked to make an advertisement to sell a pen. One student in the group held up a pen, and one member in the group pointed to it.

Other gestures included pointing to oneself (two examples) in the unassisted groups, shaking the head (one example) and shrugging the shoulders (one example) in the 
assisted groups. The two examples of pointing to oneself happened in the topic holiday; a student in this group when talking pointed to herself twice. The speech from the video, though not very clear, showed that this student was talking about her choice of a package holiday. Although not all of the students used this gesture, the two examples mentioned indicate something about a speaker. Vietnamese people, when talking about themselves, sometimes produce this gesture.

Gestures are cultural representations. In this research, nodding and shaking the head, or pointing at oneself showed agreement, disagreement or reference to oneself. In the group work, the artifacts, such as the textbook, blackboard, and classroom handouts generated gestures of pointing to attract attention and to help other members locate information.

\subsection{Discussion and Conclusion}

The results presented in this chapter showed that there are differences in the way the students in each kind of group setting discussed the assigned topics and the extent to which the students met the requirements of the tasks. There are also differences in students' perceptions of the success of the discussions. The data from the journals and interviews indicated that the students believed that they had gained knowledge of the topics through participating in group discussion, for example, knowledge about other countries, life, jobs and payment, and animals. The success of the discussions, however, was perceived differently by the students in the two kinds of groups. More assisted students considered the assisted group discussions to be efficient because they were structured, focused and helped by the senior student. The unassisted students 
reported more instances of unsatisfactory discussions because of lack of time, structure, and understanding of the required task.

The four data collection methods collected different information with regard to the discussions of the topics. The audio transcriptions showed that the unassisted groups completed fewer tasks than the assisted groups. The journals revealed the students' reports on whether the topics were interesting or not, and knowledge of the topics they gained from the discussions. The videos showed the patterns of nonverbal communication among group members through eye contact at the beginning and at the ending of a discussion. They also revealed the gestures used by the students during the discussions. The unassisted groups had irregular eye contact between the speakers and other group members. They produced more hand movements and pointed to artifacts more often than the assisted groups. The assisted groups exhibited dyadic eye contact between the senior student and the students who were speaking, or between group members. The assisted students produced fewer hand movements but more nodding of heads. The interviews touched on the students' evaluation of the group discussions, for example, how they considered whether a discussion was successful or not. In the interviews, more assisted students reported that the assisted group discussions were efficient. 


\section{Chapter 6}

\section{OPPORTUNITIES TO SPEAK}

\subsection{Introduction}

This chapter attempts to answer the second research sub-question, "What are the opportunities for speaking English in the two kinds of groups?" The data for this chapter were taken from the audio transcriptions of students' interactions in group work, students' journal entries and interview transcriptions. An utterance is defined as a unit of speech communication determined by a change of speaking subjects. As mentioned in section 1.5, Chapter 1, utterances indicate students' opportunities to speak and their participation in group work. Although the goal of the sessions under observation was to practise speaking English, utterances in Vietnamese or a combination of Vietnamese and English contributed to the meaning of the discussions. For this reason, utterances which included Vietnamese words were also counted.

This chapter begins with the presentation of the total number of all kinds of utterances. Then it continues with diagrams of the patterns of utterances. The quality of utterances was judged based on whether an utterance was on or off the assigned topic, whether it was a repetition of the textbook question, or whether it was an elaboration of it. Data from the interviews and journal entries which show opportunities to speak in group work are presented at the end of this chapter.

The audio transcriptions of group work showed that the students did not exhibit disagreement. There are some reasons for this. Firstly, the nature of the task was 
mainly to give solutions or responses to the questions from the textbook. Secondly, the students came from a cultural background influenced by a Buddhist and Confucian heritage of seeking harmony in social relationships (section 1.4.2, Chapter 1). "Elaboration" was used to describe the utterances in which the students used their own knowledge and understanding to give responses to the imagined situations and to construct ideas with other members. It has the features of both cumulative talk and exploratory talk as described by Mercer (1995) (see section 3.3.1, Chapter 3).

\subsection{English, Vietnamese and Combined Utterances}

The transcriptions were numbered based on the boundaries of utterances as defined in the introduction to this chapter. A consecutive number was given to each utterance within each topic for the transcription of each group discussion. A few utterances produced by the classroom teacher as responses to the students' questions were caught on tapes but were not counted. These utterances were brief and referred only to guidance with the tasks. The process of numbering was the same for all the topics. This allowed a comparison across topics and between the unassisted and assisted peer groups. The utterances were classified as (1) English utterances, (2) Vietnamese utterances, and (3) combined Vietnamese and English utterances.

The total utterances are presented first, followed by evaluation of their quality. The total number of all types of utterances and the means are shown in Table 2. Although the senior student's utterances were all recorded, they were not included in the opportunities to speak because the purpose was to compare utterances of the students at the same level. 
Table 2: All Utterances of Students in Unassisted and Assisted Peer Groups

\begin{tabular}{lrrrrr}
\hline & \multicolumn{2}{c}{ Unassisted students $(\mathrm{N}=60)$} & & Assisted students $(\mathrm{N}=48)$ \\
\cline { 2 - 3 } \cline { 5 - 6 } \cline { 5 - 6 } & Total & Mean & & Total & Mean \\
\hline English & 706 & 11.77 & & 685 & 14.27 \\
Vietnamese & 124 & 2.06 & & 5 & 0.10 \\
Combined & 44 & 0.73 & & 0 & 0 \\
\hline Total & 876 & 14.60 & & 690 & 14.37 \\
\hline
\end{tabular}

Note: A combined utterance includes both English and Vietnamese. The number of the unassisted students was counted by 5 students in a group in 12 sessions and the assisted groups by 4 students in 12 sessions.

Table 2 shows that there is a very small difference in the mean frequency of students' contributions according to whether the students were in the unassisted or the assisted groups. On average, an unassisted student produced slightly more utterances, 14.60 as compared with 14.37 produced by an assisted student. All students, whether in unassisted or assisted groups contributed to the discussions.

As the senior student spoke individually to each member in the assisted groups, she produced 630 utterances in 12 group sessions, which is nearly equal to all the utterances produced by all the assisted students in the same number of group sessions. Despite this, the mean of the total utterances of students in the assisted groups was only slightly lower than that of the unassisted groups (see Table 2).

However, the language of their utterances differed. On average, each assisted student produced over $20 \%$ more English utterances. The senior student always used English and the students used English to speak to her. The unassisted groups switched to Vietnamese far more often, producing 124 Vietnamese utterances whereas the assisted students produced only five utterances in Vietnamese (see Table 2). 
The two topics that generated the most Vietnamese utterances in the unassisted groups were good luck and bad luck (38 Vietnamese utterances) because when discussing this topic, the students talked about Vietnamese cultural beliefs and norms. In the session review, the students produced 42 Vietnamese utterances for planning and summarising what they had learned. The unassisted students also switched to Vietnamese when they started task planning and working out task procedures (see sequences 02, 04 and 16, Chapter 6). They also used Vietnamese to ask and explain the meanings of words in English or Vietnamese (see sequences 21, 27 and 28, Chapter 8), and to contribute ideas to the discussions. The five Vietnamese utterances produced by the assisted students were in the discussion of the topic anger (one Vietnamese utterance), and the review session (four Vietnamese utterances). When discussing the topic anger, one assisted student used Vietnamese to explain an English word. In the review session, one assisted student used Vietnamese for task planning and procedures and for asking the English equivalents of Vietnamese words.

The students in both types of groups used Vietnamese for a similar learning purpose; that is, working on task procedures and word meanings. However, the unassisted students also used Vietnamese to contribute ideas. More discussion on the role of Vietnamese is presented in section 7.2.3, Chapter 7.

Two unassisted students reported, in their journal entries, why they used Vietnamese:

At the beginning of the discussion, I did not understand the requirement of the task, so I used Vietnamese. (U-J-13)

Because we are first-year students, our speaking competence is limited so sometimes I spoke Vietnamese. (U-J-40)

One student in the interview gave the reason for using Vietnamese: 
We discussed more comfortably in Vietnamese. (16-U-I) [Translation from Vietnamese]

It is worth noting that the unassisted students produced 44 utterances of combined language whereas the assisted students did not produce any. Here is an example of a combined English and Vietnamese utterance from the transcription of session review. The student was explaining how to ask, "What do you think?"

09 S1 Moi nhom dat ra cau hoi. [Each group prepares questions.] "What do you think?" chu khong phai [not] "Do you think?"

The unassisted students produced combined utterances in similar situations to those which produced English utterances; that is, in task planning and procedures, asking and explaining words, and contributing ideas. They tended to insert Vietnamese or English words into an English or Vietnamese sentence, for example "You have good luck if your right eye nhay mat la gi vay?" This means "You [will] have good luck if your right eye winks".

\subsection{On and Off-the-assigned-topic Utterances}

Despite the fact that socio-cultural theory has been widely used in pedagogical studies, it is not a pedagogical theory in itself, rather an analysis of human behaviour in settings that are not confined to classrooms and formal education. Sociocultural theory emphasises collaboration in goal-oriented activities. The goal of each activity in the research was set out in the textbook. In this sense, whether the utterances were in Vietnamese or English, off-the-assigned-topic utterances were not relevant to the task requirement or the objective set out in the textbook, or given by the teacher. The following sequence provides an illustration of utterances which were off the assigned 
topic. The students were discussing at what age people could go to a pub in Britain.

\section{Sequence 01: Unassisted Group: Topic - Becoming an Adult}

71 S2 I think in Britain they are allowed to go to the pub at any age but not buy anything. When they go to pub in order to buy a drink or alcohol they are not allowed.

72 S5 But we don't buy a drink, I think everybody can go there.

73 S2 Yes, I think so. Even a child.

74 S3 But people go to the pub, they usually buy and drink alcohol.

$75 \mathrm{~S} 1$ If they don't buy and drink, can people go to pub and don't drink at all?

$76 \mathrm{~S} 2$ Are there people who can go to pub and don't drink at all?

77 S1 They can go to pub and meet friends.

$78 \mathrm{~S} 5$ A pub is a place with many people there but they don't want to drink alcohol. They can drink many kinds of drinks, such as Sprite, Fanta, Coca Cola...

(Laughter)

79 S2 What kind of drink do you think best?

80 S5 Sprite. And you?

$81 \mathrm{~S} 1$ Fanta.

82 S4 Bottle water.

$83 \mathrm{~S} 3$ I like the river water.

84 S2 You can when you are swimming.

Lines 79-84 were not relevant to the required discussion though they added to the atmosphere of pleasure and enjoyment.

The total utterances produced by both unassisted and assisted peer groups were then recounted based on whether they were on or off the assigned topic. The result is shown in the following table:

Table 3: On- and Off-the-assigned-topic Utterances

\begin{tabular}{lrrrr} 
& \multicolumn{3}{c}{ Unassisted students $(\mathrm{N}=60)$} & Assisted students (N=48) \\
\cline { 2 - 5 } & Total & Mean & Total & Mean \\
\hline On the assigned topic & 850 & 14.16 & 690 & 14.37 \\
Off the assigned topic & 26 & 0.43 & 0 & 0 \\
\hline Total & 876 & 14.60 & 690 & 14.37 \\
\hline Note: There were five students in the unassisted groups. The senior student's utterances are not \\
included in the table which records the utterances of four students in the assisted groups.
\end{tabular}

The unassisted students produced about $3 \%$ of utterances that were not relevant to the topics under discussion whereas the assisted students produced none. It is interesting 
that all the off-the-assigned-topic utterances were in English. In most cases, the students were joking in English. Other off-the-assigned-topic utterances were similar to lines 79-84 in sequence 01 of this section. The management utterances, for example, "Let's move to question two" was not considered as an off-the-assigned-topic utterance.

From an EFL perspective, the assisted students, on average, produced slightly fewer utterances, but more were in English and on the topic. The senior student spoke individually to each group member and so spread the opportunities to speak. Each group had a fixed time in which there were opportunities to speak. Therefore, if the senior student spoke, the assisted students had slightly less time than the unassisted groups. The unassisted students tended to have an unequal share of turns, with the students who were more assertive producing more utterances. This situation may have created a feeling among the unassisted students that they experienced fewer opportunities to speak. For example, some unassisted students reported in their journals, and answered in their interviews that they had fewer opportunities to speak in English in the unassisted groups (see sections 6.6 and 6.7, this chapter).

Alternatively, the use of Vietnamese in the unassisted groups could also have created the feeling that the students did not have as many opportunities to speak English as the assisted students did. If the purpose of the activity was to practise English, assisted groups produced more English utterances. 


\subsection{Utterance Allocation}

An excerpt in which all students produced utterances was chosen to examine how the utterances were allocated. Excerpts from three topics anger (week 1), work (week 10), and taking risks (week 12) were used to illustrate the allocation at the various points: the beginning, middle, and end of a discussion. For each illustration, both the unassisted and assisted group transcriptions are shown.

\subsubsection{Utterance Allocation at the Beginning of a Discussion}

\begin{tabular}{|c|c|c|c|c|c|}
\hline $\begin{array}{l}\text { Utterance } \\
\text { number }\end{array}$ & S1 & $\mathrm{S} 2$ & S3 & S4 & S5 \\
\hline 1 & 0 & & & & \\
\hline 2 & & & & & \\
\hline 3 & & & & & \\
\hline 4 & & & 0 & & \\
\hline 5 & & & & & \\
\hline 6 & & & & & \\
\hline 7 & & & & U & \\
\hline 8 & & & & & \\
\hline 9 & 0 & 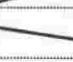 & & & \\
\hline 10 & & & & $\Rightarrow$ & \\
\hline 11 & 0 & & & & \\
\hline 12 & & & & $\rightarrow$ & \\
\hline 13 & & & 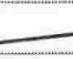 & & \\
\hline 14 & 0 & & & & \\
\hline
\end{tabular}

Note: The arrow shows the moving of each turn or utterance. It does not show the length of each utterance. The figure was adapted from Sfard \& Kieran (2001).

Figure 3: Utterance Allocation of an Unassisted Peer Group: Topic - Anger

Student S1 produced the most utterances in this excerpt: five utterances. S4 produced four utterances. S5 was the last but one person to speak and produced only one utterance. 
An illustration of utterance allocation in an assisted group follows:

\begin{tabular}{|c|c|c|c|c|c|}
\hline $\begin{array}{l}\text { Utterance } \\
\text { number }\end{array}$ & SS & S1 & $\mathrm{S} 2$ & S3 & S4 \\
\hline 1 & 0 & & & & \\
\hline 2 & & $\Rightarrow 0$ & & & \\
\hline 3 & 0 & & & & \\
\hline 4 & & $>0$ & & & \\
\hline 5 & U & & & & \\
\hline 6 & & & & & \\
\hline 7 & & & & & \\
\hline 8 & & & 0 & & \\
\hline 9 & 6 & & & & \\
\hline 10 & & & $\Longrightarrow 0$ & & \\
\hline 11 & $a$ & & & & \\
\hline 12 & & & 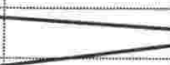 & $\Rightarrow$ & \\
\hline 13 & $9=$ & 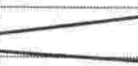 & & & \\
\hline 14 & & & & $\rightarrow 0$ & \\
\hline 15 & $0_{4}$ & & & & \\
\hline 16 & & & & $\Rightarrow 0$ & \\
\hline 17 & 04 & $E$ & & & \\
\hline 18 & & & 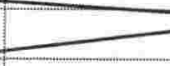 & 0 & \\
\hline 19 & 04 & & & & \\
\hline 20 & & & 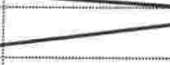 & 0 & \\
\hline 21 & 0 & & & & \\
\hline 22 & & & & 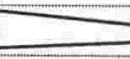 & $\Rightarrow 0$ \\
\hline 23 & 0 & 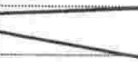 & & & \\
\hline 24 & & & $\rightarrow 0$ & & \\
\hline
\end{tabular}

Figure 4: Utterance Allocation of an Assisted Peer Group: Topic - Anger

As Figure 4 shows, the senior student spoke to each group member in turn. She controlled the discussion. Other group members took turns to speak to her. S1 produced three utterances, S2 made three utterances, S3 made five, and S4 produced only one utterance. In comparison with Figure 3, the assisted students followed a pattern of dyadic turn taking, the order of which was likely to be decided by the senior student. As figure 3 shows, the order in the unassisted group was irregular. 


\subsubsection{Utterance Allocation in the Middle of a Discussion}

Figure 5 below is an example of utterance allocation in the middle of an unassisted group discussion.

\begin{tabular}{|l|l|l|l|l|l|}
\hline $\begin{array}{l}\text { Utterance } \\
\text { number }\end{array}$ & S1 & S2 & S3 & S4 & S5 \\
\hline 31 & & & & & \\
\hline 32 & & & & & \\
\hline 33 & & & & & \\
\hline 34 & & & & & \\
\hline 35 & & & & & \\
\hline 36 & & & & & \\
\hline 37 & 0 & & & & \\
\hline 38 & & & & & \\
\hline 39 & & & & & \\
\hline 40 & & & & & \\
\hline 41 & & & & & \\
\hline
\end{tabular}

Figure 5: Utterance Allocation of an Unassisted Peer Group: Topic - Work

S5 in this excerpt produced the most utterances while S3 produced only one utterance. 
Followed is the flow of the utterances in the middle of an assisted group discussion:

\begin{tabular}{|l|l|l|l|l|l|}
\hline $\begin{array}{l}\text { Utterance } \\
\text { number }\end{array}$ & SS & S1 & S2 & S3 & S4 \\
\hline 31 & & & & \\
\hline 32 & & & & & \\
\hline 33 & & & & & \\
\hline 34 & & & & & \\
\hline 35 & & & & & \\
\hline 36 & & & & & \\
\hline 37 & & & & & \\
\hline 38 & & & & & \\
\hline 39 & & & & & \\
\hline 40 & & & & & \\
\hline 41 & & & & & \\
\hline 42 & & & & \\
\hline 43 & & & & \\
\hline 44 & & & & \\
\hline 45 & & & & \\
\hline 46 & & & & \\
\hline
\end{tabular}

Figure 6: Utterance Allocation of an Assisted Peer Group: Topic - Work

The senior student spoke to each member in the group. As it was in the middle of a discussion, the allocation of the utterances did not go in the order from S1 to S4 as in the beginning of the discussion as shown in Figure 4. Now the allocation was based on the interaction of each student with the senior student. S1 and S2 produced the same number of utterances. S3 produced only one utterance in this excerpt. S4 produced two. The interaction was almost entirely between the senior student and individual members of the group. 


\subsubsection{Utterance Allocation in the End of a Discussion}

An example of utterances in the end of an unassisted group discussion follows:

\begin{tabular}{|l|l|l|l|l|l|}
\hline $\begin{array}{l}\text { Utterance } \\
\text { number }\end{array}$ & S1 & S2 & S3 & S4 & S5 \\
\hline 63 & & & & & \\
\hline 64 & 0 & & & & \\
\hline 65 & & & & & \\
\hline 66 & & 0 & & & \\
\hline 67 & & & & & \\
\hline 68 & & 0 & & & \\
\hline 69 & & & & & \\
\hline 70 & & 0 & & & \\
\hline
\end{tabular}

Figure 7: Utterance Allocation of an Unassisted Peer Group: Topic - Taking Risks

$\mathrm{S} 2$ in this excerpt tended to be the centre of the discussion, S2 producing three utterances. S5 however produced only one utterance.

\begin{tabular}{|l|l|l|l|l|l|}
\hline $\begin{array}{l}\text { Utterance } \\
\text { number }\end{array}$ & SS & S1 & S2 & S3 & S4 \\
\hline 63 & 0 & & & & \\
\hline 64 & & & & & \\
\hline 65 & 0 & & & & \\
\hline 66 & & & & & \\
\hline 67 & & & & & \\
\hline 68 & & & & & \\
\hline 69 & & & & & \\
\hline 70 & & & & \\
\hline
\end{tabular}

Figure 8: Utterance Allocation of an Assisted Peer Group: Topic - Taking Risks

Again, this excerpt shows that the senior student was the leader till the end of the discussion. 
All six figures above show a clear difference in the patterns of utterances produced in the two types of groups. Whether at the beginning, middle, or end of a discussion, there seemed to be an assertive student in the unassisted groups who produced more utterances than the others. The direction of turn taking moved irregularly. In the assisted groups, the senior student controlled the interaction and spoke to every member in turn. Overall the assisted students had fairly equal opportunities although some had more opportunities than others to speak. Students in the assisted groups sometimes took turns to speak to each other but this pattern was not dominant. The direction of turn taking in the assisted groups was a series of dyadic interchanges between the senior student and each member of the group.

\subsection{Length of the Utterances}

The length of the utterances produced by the students in both groups was examined. As the audio transcriptions showed, one utterance might have two to three sentences but another might consist of one word, "yes" or "no", as an answer to the previous utterance. The length of the utterance was probably determined by competence in English. The utterances from the previous speaker helped to determine the thinking process in the following student. Any further analysis of length of utterance was hampered by the fact that the students used both English and Vietnamese, and the incompatible length of translation of statements in Vietnamese into English. Therefore, the research did not go further into examining the length of utterances. Instead, it focused on the content and other qualities of the utterances. The following section reports another aspect of quality of utterance; that is, whether utterances are a repetition or elaboration of the question in the textbook. 


\subsection{Repetition and Elaboration}

In nine of the 12 sessions of group work, the students had to use the questions from the textbooks to give responses. For the review session, the students did not have any questions from the textbook. In the holiday topic, the students had to imagine a vacation. The teacher gave each group some travel brochures. She adapted the task by asking the students to talk about three cities or three countries, three restaurants, and three leisure activities. The students did not have to answer specific questions from the textbook as for the other topics. In the topic advertisement, the students had to create an advertisement selling an object and again the textbook did not provide questions. Therefore, this section will focus on the remaining nine sessions.

The audio transcriptions of the nine group sessions with questions from the textbook were examined for their quality. Three categories emerged:

(1) Repetition from the textbook questions: Students read aloud the questions without making any comments or giving any further ideas. Line 2 in the following is an example of a repetition:

01 SS Ok, now let's start. You have the books alright? Yeah, we will do part four. Can you read out the first situation?

02 S1 The first one? A visitor to your home accidentally breaks your favourite vase (from text).

(2) Elaboration: Students used their own ideas to respond to the questions in the textbook. There is no repetition of the questions in the textbook. Lines 15 and 16 in the following excerpt are examples of elaboration:

14 S3 People expect to change their jobs and companies during their working lives. (Four seconds pause)

15 S5 I think this is European. 
$16 \mathrm{~S} 2 \mathrm{I}$ think in European companies people like to change jobs and companies because they like to change and maybe they get bored if they work for the same company all their lives.

(3) Repetition and elaboration: Students read out a question from the textbook and then gave some ideas as responses to the question. Here is an example:

02 S2 Hoi, hoi cau mot [Try question number one]. When a visitor to your home accidentally breaks your favourite vase (from textbook), I think I will try to buy another one later.

S2 repeated the question from the textbook and added an elaboration, "I think I will try to buy another one later."

The audio transcriptions also showed other kinds of utterances, for example, "Can we move to question two?" or "Can you read it?" These utterances function as management questions. Some utterances functioned as explanations of vocabulary which will be discussed in the Chapter 8 . The senior student's utterances were almost always in the form of questions. Table 4 shows the quality of utterances examined in the three categories: repetition, elaboration and repetition, and elaboration. It does not include the management utterances or explaining utterances for vocabulary.

Table 4: Quality of Utterances of Students in Unassisted and Assisted Groups

\begin{tabular}{|c|c|c|c|c|}
\hline \multirow[b]{2}{*}{ Utterance } & \multicolumn{2}{|c|}{$\begin{array}{l}\text { Unassisted students } \\
\qquad(\mathrm{N}=45)\end{array}$} & \multicolumn{2}{|c|}{$\begin{array}{l}\text { Assisted students } \\
(\mathrm{N}=36)\end{array}$} \\
\hline & Total & Mean & Total & Mean \\
\hline Repetition from the textbook & 61 & 1.35 & 18 & 0.50 \\
\hline Repetition and elaboration & 13 & 0.28 & 1 & 0.03 \\
\hline Elaboration & 357 & 7.93 & 382 & 10.61 \\
\hline
\end{tabular}

Note: The total utterances in this table were counted from the audio transcriptions of nine topics only. The number of the unassisted students was counted by 5 students in a group in 9 sessions and the assisted groups by 4 students in 9 sessions. The senior student's utterances were not included in Table 4. 
Table 4 shows that the unassisted students produced more utterances repeated from the textbook, a mean of 1.35 per student as compared to 0.50 in the assisted groups. The apparent reason for the fewer repetitions in the assisted group is that the senior student managed the discussion by sometimes reading out the questions from the textbook. When the unassisted students repeated the questions from the textbook, they sometimes also added their own ideas and expanded the questions. As a result, 13 utterances of this kind were found in the audio transcriptions of the unassisted groups as opposed to only one utterance in the assisted groups. The assisted students had a rather higher mean of 10.61 for the elaborated utterances in comparison with that of 7.93 of the unassisted students. This represents one third more elaborated answers from assisted students.

Table 4 also indicates that the assisted students produced more cumulative and exploratory talk. Mercer (1995) discussed talk of these kinds (see section 3.3.1, Chapter 3). Because the senior student helped with the management of the group work, for example, by showing the assisted groups what to do and where to look for information, the assisted students appeared to have more time and opportunities to elaborate their ideas. They expanded and built upon each other's utterances. An alternative explanation could be that the senior student's probing questions facilitated the production of cumulative and exploratory talk among the assisted students. In the unassisted groups, the students produced more utterances which repeated earlier statements in order to fill in the pauses when they had to think of responses to the questions in the textbook. 
The audio transcriptions were also examined to see whether there were examples of private speech produced by individual students speaking to themselves. As pointed out in section 3.3 , Chapter 3 , the use of private speech occurred very infrequently and not often enough to warrant a separate category.

\subsection{Journals}

The students were asked in their journal, "Did you have an opportunity to speak in English today. If yes, what did you say? If no, please explain why not?" The purpose of this question was to help the students recall the lesson, and the general background of the group work in order to answer further questions in the journals. The question also checked whether the students believed they had opportunities to speak. The students in both types of groups reported that they had had an opportunity to speak English in the group session. However, not all of them reported what they had said. Five students in the unassisted group confirmed that they had had an opportunity to use English to participate in the discussion. In comparison, 18 students in the assisted groups reported having had an opportunity. Seven students in the unassisted groups and three in the assisted groups reported that they had had an opportunity to speak, but not much. The reports from some students in the unassisted groups were general. Some examples follow:

It was a good chance for me to speak English. (06-U-J)

I had a chance to express my opinions, thoughts on the topic, and to practise communicative skills. (16-U-J)

In the assisted groups, some students gave more detailed responses, for example, more opportunities to speak with the help of the senior student (six responses), and 
opportunities to speak given by other group members (two responses). Others wrote about the group work as a good chance to state their opinions comfortably and easily. Some examples follow:

The senior student created situations so that everyone in the group could speak. (02-A-J)

The assistance of the senior student helped us to speak more English. (18-A-J)

Friends helped me to speak and understand by speaking slowly and giving me opportunities to speak. (15-A-J)

I could speak as much as I wanted. My friends waited for me and created an opportunity for me to speak. We speak more than we spoke before. (41-A-J)

However, one student stated,

I did not have as much opportunities as in the unassisted group because the senior student tried to share opportunities to speak to every member. (35-A-J)

Seven unassisted students reported that they did not speak much. The reasons included not understanding the requirements of the task (three responses), not feeling well (one response), not preparing for the lesson (one response), other members spoke too much (one response), limited time and lack of English competence (one response), and using Vietnamese (one response).

Three assisted students reported they did not speak much. One said that other members had said already what he/she intended to say. The other two claimed that the topics advertising and working styles were difficult to discuss.

As reported in the journal entries, more students considered assisted group work as a situation where they could readily engage in discussion and use more English. What the senior student in the assisted groups said seemed to be one of the factors that enhanced students' interaction in English although one student (35-A-J) would probably have 
spoken more without the senior student. The responses of the students indicated that they understood the convention that there needed to be opportunities to speak in English and to share turn-taking equally.

\subsection{Interviews}

In the interviews, I asked the students, "In what ways was your experience in the group work with the senior student different from without her?" Twelve students (four unassisted students, and eight assisted students) reported the opportunity to speak in the group work, though they were not overtly asked about it. Their answers, which were translated from Vietnamese, indicated that having an opportunity to speak in groups was a key issue and the students perceived the importance and difference of the opportunity to speak in the two kinds of group settings. Here are some examples from the interviews with the unassisted students:

I spoke less in the group without the senior student. (04-U-I)

More active students contributed ideas. Shy members did not contribute. (06-UI)

The unassisted students did not follow a regular speaking order whereas the senior student tended to allocate turns. Therefore, the students who were shy were likely to consider that the assisted groups gave them more opportunity to speak. Following are some examples from the interviews with the assisted students:

The senior student gave suggestions so we thought more and we spoke more. (05-A-I)

She helped slow students to speak. (12-A-I)

The assisted students depended somewhat on the initiative of the senior student as to when they spoke. 


\subsection{Discussion and Conclusion}

This chapter addresses the second research sub-question on the opportunities for speaking English in the two kinds of groups. The results showed that in general, there was a slight difference in the average number of utterances produced by a student in the two group settings. However, the quality of the utterances varied according to whether the students were in the unassisted or assisted groups. The unassisted groups produced fewer English utterances, but more Vietnamese and combined language utterances than the assisted groups. The assisted groups produced more English utterances, very few Vietnamese utterances, and no combined language utterances. The unassisted groups made some off-the-assigned-topic utterances whereas none of such kind of utterance was made in the assisted groups. The pattern of utterance allocation in group interactions in the unassisted groups was irregular whereas in the assisted groups, the pattern was dyadic and between the senior student and each member of the group.

The different group settings and composition created different opportunities to speak. Although the students, especially those in the unassisted groups, used both English and Vietnamese in the interaction, it seemed that they tended to define the opportunity to speak as the opportunity to speak in English. This does not mean Vietnamese or combined language utterances did not contribute to the discussions. The role of Vietnamese and combined language utterances will be discussed in depth in the next chapter.

The results from all sources revealed an anomaly. The students (mainly the students when in the unassisted groups) perceived the goal of using English in group work but 
they sometimes needed to use Vietnamese in order to express themselves. As a result, they considered they had more opportunities to speak in the assisted groups. 


\section{Chapter 7}

\section{PEER MEDIATION THROUGH ORAL LANGUAGE}

\subsection{Introduction}

This chapter addresses the third research sub-question, "How do English and Vietnamese languages, if used, play a mediational role in the discussions?" It presents sequences of interaction from the audio transcriptions to illustrate peer mediation through the use of both English and Vietnamese. The focus on peer interaction allows the examination of group work which was described as a unit of analysis of the current study. The sequences were chosen from different topics and at different stages of the discussions. Most sequences are presented in pairs, one from an unassisted peer group and the other from an assisted peer group. Unsuccessful sequences of negotiation are also presented to examine what factors affected the discussions.

\subsection{Negotiation in the Group Work}

Participation is always based on situated negotiation and renegotiation of meaning in the world. This implies that understanding and experience are in constant interaction, indeed, are mutually constitutive. (Lave \& Wenger, 1996, p. 146)

As pointed out in section 4.12.1, Chapter 4, the students' participation in group interaction is examined within the category of negotiation, focusing on the mediational functions of speech as it is used as a tool for constructing meanings and sharing understanding. The category of negotiation reported in this chapter includes: 1) The negotiation of task planning and procedures, and 2) The negotiation of understanding. See definitions of the categories in section 4.12.1, Chapter 4. 


\subsubsection{Negotiation of Task Planning and Procedures}

Among many sequences recorded, those from the topics holidays, urban and rural, and advertisement were chosen to illustrate how the negotiation of task planning and procedures occurred in the two kinds of groups. In the topic holidays, the students had to decide where to take a vacation. The classroom teacher, however, adapted the task as set out in the textbook and required the students to talk about three cities, or countries, three restaurants and three leisure activities which the students would like to do on their vacation. The class teacher gave travel brochures to the students to share.

\section{Sequence 02: Unassisted Group: Topic - Holidays}

01 S1 What will we do?

02 S2 Toi Ha Lan duoc khong? [How about Holland?] Which country would you like to go on your vacation?

03 S1 On your holidays.

04 S3 Hoi nhu vay? [Should we talk like that?]. Hoi thu. [Yes?] Minh khong hieu. [I don't understand.] Quyet dinh cung nhau di dau do va lam gi. [We have to decide together where we want to go and what activities to do.] (Inaudible)

05 S2 Which country would you like to go?

(Inaudible)

$06 \mathrm{~S} 1$ Di Ha Lan. Chon mot thanh pho. [Holland. Choose a city!] (Five seconds pause)

07 S4 Hoi co giai thich them. [Let's ask the teacher for more information.] Minh noi chua duoc dau. [I don't think we can talk now.] Three cities, three countries, three activities and restaurants, three activities.

$08 \mathrm{~S} 3$ Hoi co minh chon duoc ba nuoc hay sao. [Ask the teacher if we can discuss three countries.] Minh co the chon ba dat nuoc hay sao? [Can we choose three countries?]

(10 seconds pause)

09 S2 Di Trung Quoc [Go to China]

10 All China.

11 S2 Excuse me, should I choose three countries?

$\mathrm{T}$ I want you to choose three and decide on one. You can have three cities if you want, but one is ok. You have to see things when you are there. You can have one city if you want, but three will be better. You do things together.

12 S4 Thao luan ba noi de chon mot [We have to choose one out of three.]

13 S2 Minh se co ba cai nhung minh se chon mot de di. [We have three but we have to choose one.] 
14 S4 Ba noi minh thich noi nao thi minh di. [Three places but we choose a place which we want to visit.] Minh se noi minh thich di mot noi nao do [We will say that we would like to go to a certain place.]

15 S3 Noi di. [Let's talk!]

16 S2 I think China is an interesting place to visit.

S1 started the discussion but was not clear about the required tasks (line 01). S2 used combined English and Vietnamese to suggest a country and asked others for their opinions (02). S3 was listening but did not understand completely what the task required. S3 used Vietnamese to negotiate a collective decision (04). S2 broke in and asked other students about a place to visit (05). S1 chose Holland as a country but suggested they talk about a city (06). S1 used Vietnamese in this utterance. S4 was still confused about what to do and suggested asking the classroom teacher for more details (07). S4 used both English and Vietnamese. S3 listened to S4 but was not sure about the required tasks (08). S3 used Vietnamese. S3 wanted to seek help from the teacher. S2 suggested going to another country different from S3 and S4's suggestion (09). The group then agreed to choose China (10). S2 asked the teacher what was required (11). S4 repeated the teacher's explanation in Vietnamese (12). S2 added more information to S4's explanation (13). S4 confirmed what the group had to do (14). S3 encouraged other students to speak (15). The chain of interaction among S4-S2-S4-S3 was in Vietnamese. S2 responded by identifying China (16). The discussion continued.

In this sequence, the students were confused about what they were supposed to do and they used mainly Vietnamese. S5 did not say anything. When the students failed to ascertain what to do, they sought help from the teacher. The students then started planning again. Four members of the group participated and finally they started the discussion. The task was shared. The negotiation of the task planning and procedures 
required the collective participation of the whole group. The interaction among the group members and interaction in Vietnamese helped the students to figure out what they had to do.

The following sequence illustrated the negotiation of task planning and procedures in an assisted group.

\section{Sequence 03: Assisted Group: Topic - Holidays}

01 SS OK, can we start now? Please tell which picture you want the first, the second or the third? You have to choose three countries, three cities, three activities, and three restaurants. Which place do you want to go? You can choose from this magazine.

02 S1 I want to visit Dalat. I want to visit Love Valley.

03 SS Love Valley. Wow! So where else in Dalat do you want to visit?

The senior student explained what was required and suggested what the students should do. She told other students where to look for the information (01). S1 provided an answer to the suggestion of the senior student (02). The senior student praised S1's response and suggested more specific relevant answers (03). The senior student's explanation helped the students understand it within the first minute of discussion. There was no lengthy process of negotiation about what to do. S1 immediately chose Dalat, a famous holiday resort in Vietnam, as a travelling destination.

In sequence 04 , the students were required to prepare two questions for each topic that they had studied. The teacher told the class that she might use these questions again in their mid-term oral English exam. 


\section{Sequence 04: Unassisted Group: Review Session}

01 S1 Mot nhom hai cau hoi. [Each group has to prepare two questions.]

02 S2 Noi tieng Anh di. [Let's speak English]. (Noisy background, chatting in Vietnamese, 20 seconds)

03 S3 Moi nhom hai cau. [Two questions for each group.]

04 S1 Moi nguoi dat hai cau hoi. Dat ca nhom roi chon ra mot hoac hai cau. [Each person has to prepare two questions and then the group has to choose one or two questions for each topic.]

$05 \mathrm{~S} 2$ Tat ca moi nguoi deu dat cau hoi. [Everyone in the group has to prepare questions.]

06 S4 Moi nhom dat hai cau ve moi chu de. [Each group prepares two questions for each topic.]

07 S5 Khong phai mot nguoi dau. Ca nhom dat ra roi hoi. [It does not mean that each person has to prepare two questions. The whole group has to prepare questions.]

$08 \mathrm{~S} 3$ Tat ca dat roi chon ra mot hoac hai cau. [Everyone prepares questions and then we choose one or two questions].

(Six seconds pause)

09 S1 Moi nhom dat ra cau hoi. [Each group prepares questions.] "What do you think" chu khong phai [not] "Do you think".

10 S2 Ban dat cau hoi di. [Please ask questions!]

11 S4 Mau len, le len. [Hurry up!]

(Five seconds pause)

12 S3 Noi chu de nay di. [Let's talk about this topic] (The student was focusing on the topic in the textbook and produced a question). Among good health and money, which one do you choose?

13 S5 Minh noi cai nay roi. [We have studied this.] (20 seconds pause)

14 S3 Chon di. [Decide, please.]

$15 \mathrm{~S} 1$ Health.

$16 \mathrm{~S} 2$ Ghi vao. [Write it down!]

$17 \mathrm{~S} 1$ Ghi lai cau hoi nay ha? [Write down this question?] (16 seconds of pause)

$18 \mathrm{~S} 3$ How are good women in a modern life?

The students in this sequence produced only three utterances in English (09, 12, and 18). Utterances 12 and 18 were the questions that the students were required to prepare. The other 15 utterances were in Vietnamese. The Vietnamese utterances were all about what the students had to do. Vietnamese helped their group management and the process of figuring out what they had to do. All the students spoke in this sequence. For example, S1 started the discussion (01). S3 reminded the required task (03). S4 and S5 wanted the group to be more specific about the task which asked each group to prepare 
two questions for a topic (06 and 07). S2 managed the task by reminding the other students of what they had to do $(02,10$ and 16$)$. Each student contributed to the process of working out the required task. There were long pauses. The students needed time to figure out what they had to do and it can be noted that they then recorded their questions in writing.

Preparing questions for the review session was also carried out by an assisted peer group. Following is the transcription:

\section{Sequence 05: Assisted Group: Review Session}

01 SS OK, are you ready? Can you tell me what units you have already done? Can you tick the units you have already done? You should speak English.

$02 \mathrm{~S} 1$ Noises and sound.

03 S2 Animals.

04 S3 Good luck and bad luck.

$05 \mathrm{~S} 1$ Fears and phobias.

$06 \mathrm{~S} 4$ Women and men.

07 S2 Nhung tui minh chua hoc women and men. [We have not studied the topic women and men]. Len noi co. [We have to tell the teacher about this.] (One student stood up and told the class teacher.) (30 seconds pause)

08 SS First, the topic love. Tell me what do you want to talk about the topic love?

$09 \mathrm{~S} 1$ The role of love in life.

10 SS Now you have to think of questions for each topic, OK? Which questions do you want to ask about the topic love?

11 S2 What do you think about the role of love in your family?

In comparison with the unassisted group in sequence 03, the assisted group was very organised because the senior student told them where to start and what information to look for $(01)$. The group then contributed to the starting process by listing what they had learned (02-06). When they realised that they had not studied the topic women and men, they told the classroom teacher. The senior student orchestrated the discussion, for example, "First, the topic love" (08). She also posed questions so that the students 
could think of their own questions for each topic. S2 made one utterance in Vietnamese (07) when she was reporting to the group that the topic had not been covered. Other students used English and the senior student encouraged them to use English (01). Although the senior student controlled the group process, other members contributed to the discussion planning.

In the topic urban and rural, the students were required to talk about the differences between the city and the country. The textbook provided a list of categories. The teacher asked the students to choose only four of the categories to discuss.

\section{Sequence 06: Unassisted Group: Topic - Urban and Rural}

11 S4 What do you think about health in the country?

12 S1 I don't want to say about health in the country. I want to say about education. The children in the country do not have good conditions to study.

13 S4 But we are discussing health.

14 S1 No, all the differences in the country and city.

$15 \mathrm{~S} 4 \mathrm{I}$ think that our health in the country is very good.

$16 \mathrm{~S} 1$ Sorry, do we have to do part two? We need to talk about the differences between city and country. We can discuss about health, holidays, clothes, stress, people and rainy weather.

17 S2 No, we discuss all the differences between city and country.

$18 \mathrm{~S} 1$ I think the teacher told us to do that.

19 S2 When did she tell you? Yesterday?

20 S1 Some minutes ago. Did you hear that information?

$21 \mathrm{~S} 3$ No.

22 S5 I have no ideas.

$23 \mathrm{~S} 1$ So we talk about all the topics. Continue.

The students were confused about the required task. They had been asked by the teacher to choose four categories among the list in the textbook. However, their disagreement was about task procedures, and did not show the characteristics of "disputational talk" (Mercer, 1995). They argued about what should be included in the discussion. They referred to the teacher as an authority. S1 was rather dominant and 
decided what the group should do. The negotiation of task planning in this case was not successful and the unassisted group did not reach the proper solution. S4 wanted to focus on the task but S1 refused to follow S4's suggestion (12). S1 and S4 continued to negotiate about what should be discussed and then S2 broke in and referred to the classroom teacher for information (18). S1 checked with other members and asserted authority by suggesting that the group move on to discuss all the categories (23). The students in this sequence used only English to negotiate the required task. They had difficulty when there was more than one thing to choose from and when they might have to make more than one choice.

Here is the task planning in an assisted group.

\section{Sequence 07: Assisted Group: Topic - Urban and Rural}

$01 \mathrm{SS}$ Ok are you ready?

$02 \mathrm{~S} 1$ Yes.

03 SS You do part two. You just choose four categories. Ok, what categories would you like to talk? How many categories are there?

04 S2 Six.

05 SS We choose only four. Which ones do you want to focus on?

$06 \mathrm{~S} 2$ Health.

07 SS How about the next one? Do you want to talk about holidays?

08 All Yes. (All students reply.)

09 SS What else?

10 S1 People.

11 SS Yes, and the last one.

12 S3 The rainy weather.

$13 \mathrm{~S} 1$ That's all.

The senior student explained to the students what they had been asked to do and invited them to choose the categories. She also gave a suggestion on one category (07).

The negotiation of task planning generally occurred at the beginning of each discussion. On this measure, the sequences show a clear difference between the two 
kinds of groups. The unassisted students spent more time on this kind of negotiation and used more Vietnamese. The assisted students spent less time and hardly used Vietnamese. The senior student assisted the students to start the discussion. She explained the required task and showed which part of the textbook should be read. She gave the students a clear idea of what to do.

There were also examples of negotiation of task planning and procedures at other stages of the discussion, for example, in the middle, or near the end of the discussion. This kind of negotiation occurred more often in the unassisted groups than in the assisted groups. Following is an illustration of task negotiation near the end of the discussion:

\section{Sequence 08: Unassisted Group: Topic - Urban and Rural}

$53 \mathrm{~S} 1 \mathrm{We}$ can write later. Now we have to do part five. We do not have enough time to write down.

54 S2 We should do like this: one person in the group presents one idea and we will choose ideas.

55 S1 Now we can do part five.

$56 \mathrm{~S} 2$ We have no ideas how our group will present. One member in our group must stand up and present our ideas to our teacher.

S1 and S2 were negotiating first what to do and how they would present their ideas to the teacher and the class. The students were using English in this discussion.

An example of negotiation of task procedures in an assisted group follows:

\section{Sequence 09: Assisted Group: Topic - Advertisement}

26 SS So think of the way to make the whole advertisement from the start till the end. You can imitate the ways they advertise on TV.

27 S2 I don't think there are advertisements on clothes on TV.

28 SS Do you think that you should move closer? Do you think it is easy to advertise clothes?

29 S1 No.

30 SS Why do you choose clothes? I think it is rather difficult. 
31 S4 Maybe we should change.

The students had planned what to advertise in the early discussion. They wanted to advertise jeans and a T-shirt. The senior student then suggested that it was rather difficult to advertise clothes. They then discussed the possibility of changing to another product (31). They discarded the idea of clothes, accepting the senior student's authority.

\subsubsection{Negotiation of Understanding}

The following sequences were chosen from the topics anger, good luck and bad luck, and work. The task of sequences 10 and 11 was set out in the textbook, "Look at these situations. How would you behave in each situation? Would you get angry? How would you show your anger? Or would you try to control yourself?" In the first situation, the students were asked how they would respond to a situation in which a visitor accidentally breaks their favourite vase.

\section{Sequence 10: Unassisted Group: Topic - Anger}

$01 \mathrm{~S} 1$ Mau len. [Hurry up!]

02 S2 Hoi, hoi cau mot [Try question number one]. When a visitor to your home accidentally breaks your favourite vase (from textbook), I think I will try to buy another one later.

03 S3 When a visitor to my home accidentally breaks my favourite vase, I will try to make the visitor feel comfortable when they have break carelessly.

04 S4 Yes, if a visitor to my home accidentally breaks my favourite vase although I am very sad but I try to make the visitor not to make my visitor feel uncomfortable.

$05 \mathrm{~S} 3$ Uncomfortable?

06 S4 Khong duoc thoai mai. [Uncomfortable.] (Two seconds pause)

07 S2 When a visitor to my home accidentally breaks my favourite vase, I think that may be a big problem, so we must think about the problem and have best solutions to choose to solve when we have made them.

$08 \mathrm{~S} 1$ I think you need to answer one two three four five (five seconds pause) (Inaudible). Question two. 
The students in this episode used the cues provided in the textbook as a resource. Each student produced a solution for the situation. S1 adopted the role of group leader. At first, S1 suggested a plan of the task and later reminded other students about keeping to the plan (01 and 08). S2 produced the first response (02). S3 expanded and elaborated S2's idea and added another idea that the visitor may have been careless (03). S4 understood what S3 meant and expanded the discourse by adopting the idea of making the visitor comfortable (04). S4 showed understanding of the previous statement and picked up the idea of comfort from S3 to provide a response different from the ones given earlier. S3 obviously followed what S2 and S4 had said but asked for the meaning of a word (05). S4 broke in to help explain the word in Vietnamese (06). A linked discussion between S3 and S4 stimulated another answer from S2 (07). There were four different solutions to the imagined situation. Although S1 took on the role of leader, his/her function was to negotiate task planning and procedures. For example, S1 suggested answering task questions number one, two, three, four, and five as set out in the textbook and moving on to question two (08). Other students followed the rule that everyone in the group had to contribute ideas. All the students understood this protocol since they were not school beginners as in the McDonald et al.'s (1991) study and they already knew the behaviour expected of them. S5 did not speak in this first sequence.

The same situation, "What to do if a visitor accidentally breaks your favourite vase?" was also discussed by the assisted group. The transcription follows:

\section{Sequence 11: Assisted Group: Topic - Anger}

01 SS Ok, now let's start. You have the books, alright? Yeah, we will do part four. Can you read out the first situation?

02 S1 The first one? A visitor to your home accidentally breaks your favourite vase (from textbook).

03 SS What will do you then?

04 S1 Maybe I will get angry.

05 SS Angry? Why? 
06 S1 Because it's my favourite vase. Maybe I can't buy another one.

07 SS So how about you?

$08 \mathrm{~S} 2$ I will get rather angry. It is my favourite vase.

09 SS You mean you will get a little angry. Why do you get a little bit angry?

$10 \mathrm{~S} 2$ Because it's my favourite vase.

11 SS And that means you can't buy another one. Will you get angry also?

12 S3 I don't think so. Because he is my guest. You can't be angry with him.

13 SS So if you don't get angry what will you do then? What is your behaviour then?

14 S3 I think it is ok.

15 SS Will you smile? (Laughter)

$16 \mathrm{~S} 3$ Yes.

$17 \mathrm{SS}$ Will you tell him to buy you another one?

$18 \mathrm{~S} 3$ Yes.

19 SS Yes?

20 S3 No.

21 SS You mean you just smile? How about you?

22 S4 I agree with Hung. If he breaks my favourite vase, I can buy another one.

23 SS Do you get angry with the guest? Just smile? So which behaviour do you like the most?

24 S1/S2 Hung's behaviour.

25 SS Just smile. Don't get angry. How about the second situation? Can you read out the second situation?

The senior student started the discussion (01). S1 read out the situation from the textbook and the senior student expanded it with a task-focused question (03) "Angry? Why?" The senior student's question sought more detail from S1's response (06). The senior student then focused on another student (07). She asked S3 another "why" question to S2 (09). She tried to elicit a different idea from another student (11). S3 gave a negative answer to the senior student's question and S1's answer and provided a new response (12). The senior student tried to elicit a further answer from S3 (13-15). When S3 had four responses, the senior student addressed another student (21). S4 said that she agreed with an earlier speaker thus indicating that she had been listening to the exchange. When all the students had given a response, the senior student called for a conclusion (23) which produced answers from S1 and S2 (24). The senior student then confirmed the responses that the students had given and suggested moving to the next 
situation (25). The senior student engaged each student in turn. She asked for reasons for the answers and all students used English to contribute ideas.

The students in the following sequence were discussing the topic "What brings good luck or bad luck in your home country".

\section{Sequence 12: Unassisted Group: Topic - Good Luck and Bad Luck}

42 S2 If the person visits your house in the New Year is not good.

Nguoi ma khong tot. [The person who is not good.]

$43 \mathrm{~S} 1$ The first person to visit you in the New Year is a bad person, you will have bad luck in this year but if he is a good person you will have good luck.

(Two seconds pause)

44 S4 Nguoi co tang thi khong toi nha nguoi khac. [If your family has just had a funeral, you should not visit other families.]

45 S2 Nguoi ma co tang thi khong duoc toi nha nguoi khac vao Tet. [If your family has just had a funeral, you should not visit other families on Tet Holidays.]

46 S3 If you have funeral, if your family has a person that died, you must not visit other houses in Tet.

47 S2 On Tet holidays because you will bring bad luck.

48 S5 If you dream about bad things, you will have good luck.

S2 started with the idea of good luck associated with the first visitor on New Year's Day which is very important in Vietnam. Vietnamese people expect cheerful and fortunate people to visit them on New Year's Day. S1 adopted the idea and talked about a good person. S4 expanded with more detail (44). S2 and S4 shared a sentence and a single idea (44-45). S3 translated S2 and S4's Vietnamese sentence into English. Listening to the exchange between S4 and S2 in Vietnamese about the concept of good luck and bad luck associated with a "funeral", S3 understood that there was a word in English to express the idea. S3 then used English and shared a sentence with S2 (4647). 


\section{Sequence 13: Assisted Group: Topic - Good Luck and Bad Luck}

69 S3 The first day of the New Year, I see a woman.

70 SS The first one to enter your house to visit your house on the first day of a new year is a woman. Do you think that it is good luck or bad luck?

71 S3 I don't know.

72 SS How about you? Do you think it is bad luck?

$73 \mathrm{~S} 4$ It depends on the person. If the person has good relation with your family, you will have good luck. But if the person has bad relation with your family, you will have bad luck.

74 SS Do you agree with her?

$75 \mathrm{~S} 1$ Yes.

76 SS What else do you think is good luck or bad luck in your life?

Similar to the unassisted group, the assisted students in this sequence talked about the first visitor on New Year's Day, illustrating its cultural importance. S3 started and the senior student asked other students questions. S4 answered in detail (73) and S1 agreed with S4 when asked for agreement by the senior student. The senior student changed the discussion to another topic.

The students in sequences 14 and 15 were discussing the topic work. The textbook explains that, "Different countries have different styles of working. For example, Japanese companies are very different from those in Europe. Do you think these statements are more likely to be made about Japanese or European companies?" The textbook gives a list of statements.

\section{Sequence 14: Unassisted Group: Topic - Work}

14 S3 People expect to change their jobs and companies during their working lives.

(Four seconds pause)

15 S5 I think this is European.

16 S2 I think European people like to change jobs and companies because they like to change and maybe they get bored if they work for the same company all their lives.

17 S4 They want to have a new life.

$18 \mathrm{~S} 1$ The director eats in the canteen with the workers. 
S3 read out the statement from the textbook. S5, S2, and S4 stated what they knew, thereby sharing their knowledge. S2 gave details and S4 elaborated S2's idea. S1 broke in to direct the group to another statement in the textbook.

\section{Sequence 15: Assisted Group: Topic - Work}

37 SS How about the next one? People expect to change their jobs and companies during their working lives (from textbook). This is different from the first one.

38 S2 European people want to make money so they want to change their jobs. Sometimes their work is boring or they get low salary so they change their work to earn more money.

39 SS Do you think so?

40 S1 European people want to change their jobs they feel more interesting than doing the same job.

41 SS Do you think that they change their jobs for interest or for money?

42 S1 I think both.

$43 \mathrm{SS}$ Do you have any more ideas?

44 S4 No.

45 SS Let's move to the next one.

The senior student read out the statement. S2 and S1 contributed ideas. S1 added more information to S2's answer (38-40). The senior student tried to draw S4 into the discussion but S4 said only "no".

The sequences presented in the section demonstrated the peer mediated learning through oral language. The students expanded, elaborated and adopted each other's ideas and those suggested by the senior student (sequences 10,11 and 12). The students asked questions, provided interpretation, and answered the questions from the textbook and from other members. They exchanged ideas and promoted thought in response to the questions from the textbook (sequences 14 and 15). The senior student challenged the students in the assisted groups to think about the situation at hand (sequence 11). The processes of using the oral language in these sequences have the feature of mediated learning as reported in the study by Gillis (2003) (see section 3.3, Chapter 3). 
The shared learning process also included reproducing knowledge acquired by growing up in Vietnam (sequences 12 and 13). Because the utterances were produced in a chain in which students took turns, it became harder for students who spoke later if this idea had been used by others.

The audio transcriptions of all the unassisted group work show eight examples of negotiation of task planning and procedures as opposed to five examples in the transcriptions of assisted group work. The unassisted groups produced 64 examples of negotiation of understanding whereas the assisted groups produced 86 .

With regard to the negotiation of task planning and procedures, in the topic vacation, the unassisted group produced three episodes of negotiation of this kind because they were not clear about what they had to do. They collectively tried to figure out what to do to complete the task. Topic good luck and bad luck produced one example. There were two examples of this kind of negotiation in the review session and in the topic rural and urban. The negotiation of task planning and procedures in the unassisted groups occurred most often at the beginning at the discussion. During the discussion, if the students were confused about the task procedures, the students again collaborated to work out what to do. They usually used Vietnamese and English speech when working together. They were sometimes successful and sometimes unsuccessful when they worked on task procedures.

By comparison, the assisted groups produced five examples of negotiation of task planning and procedures. There was one example at the beginning of the discussions of the topics vacation, review and urban and rural. The topic advertisement produced one 
example at the beginning and another one in the middle of the discussion. The negotiation was between the senior student and the other students when they were setting up the task. In this circumstance, she spoke to the group rather than an individual and individuals in the groups took turns to talk to her. The senior student helped them to be precise about what needed to be done. In the topic advertisement, the students changed topic and they had another example of negotiation of task planning and procedures in the middle of the discussion. While the unassisted group negotiated an understanding of the required tasks first and then moved on with task procedures, the assisted students moved more rapidly because, with the help of a senior student, they understood the required tasks, and they just needed to decide in consultation with the senior student the next steps to take. The unassisted students took more time over negotiation of task planning and procedures.

In each topic, for both kinds of groups, there were examples of negotiation of understanding. The topics for which the unassisted groups produced the most examples of this kind of negotiation were good luck and bad luck, and work (eight examples each topic), followed by anger (seven examples), animals, traditional and modern, and taking risks (six examples in each topic), becoming an adult (five examples), and vacation, urban and rural, advertisement (four examples each topic). There were four examples of negotiation of understanding in the review session and two examples in the topic job and salary.

In the assisted groups, the frequency of negotiation of understanding in all the discussions also varied across the topics. The topic work produced 14 examples, traditional and modern, 12 examples, and good luck and bad luck, 10 examples. The 
review session and the topic urban and rural each produced eight examples. There were also examples from the remaining topics: animals (seven examples), anger, and taking risks (six examples), vacation (five examples), advertisement and becoming an adult (four examples), and jobs and salary (two examples).

\subsubsection{Mediation Through the First Language}

The audio transcriptions (for examples, sequences $02,04,10$ and 12 in this chapter) showed that the unassisted students used oral Vietnamese in the process of group work (see also Table 2, Chapter 6). The assisted groups produced only five Vietnamese utterances in the whole transcriptions of all the topics. At the beginning of discussion, the students in the unassisted groups used Vietnamese more often for task planning and procedures (sequences 02 , and 04) or when they were negotiating and sharing knowledge which could happen at any stage of the discussion (sequences 10 and 12). Another example of the use of Vietnamese in an unassisted group follows:

\section{Sequence 16: Unassisted Group: Topic - Jobs and Salary}

35 S3 Next.

$36 \mathrm{~S} 1$ What can you say about how different jobs are valued by society? (From textbook). Minh khong hieu cai nay lam. Value la co gia tri trong xa hoi a? [I don't understand this clearly. Valued by society?]

37 S4 Ban co the noi gi vi du co nghe lam duoc nhieu tien. [What you can say about, for example, some jobs that can earn a lot of money.]

38 S2 Mot nguoi co the lam gi trong xa hoi. Viec kho khan hay la nguy hiem va duoc tra nhieu tien hon. [What can a person do in society. An easy job or a dangerous job which is paid higher.]

39 S5 Duoc dao tao khong? [Is there training?]

(Eight seconds pause)

$40 \mathrm{~S} 1$ Khong quyet dinh gia tri tien luong dau. [It does not determine the pay.]

$41 \mathrm{~S} 2$ Co chu. [Yes, it does.]

(Four seconds pause)

$42 \mathrm{~S} 1$ The job requires training moi co gia tri. [A job that requires training has more value.]

43 S3 I think they receive training, they can do jobs.

44 S5 Dao tao thuong cho nhung viec do. [Training is required for some jobs.] 
45 S1 Minh khong hieu. Cong viec va gia tri khac nhau trong xa hoi. [I don't understand. Different jobs and how they are valued by society.]

(End of tape.)

S1 was confused about the concept of how jobs are valued by society. The whole group then helped to explain it. They elaborated the concept (37, 38 and 39). They used Vietnamese. It could also be that their English was not sufficient for the task. On occasion, oral Vietnamese helped the production of oral English. For example, the idea in Vietnamese by S5 about payment and a job that requires training facilitated the use of English by S1 and S3 to express the related idea.

In sequences $02,04,10,12$ and 16 , Vietnamese clearly helped the students to negotiate task planning and procedures, and to find answers to the questions in the textbook. The Vietnamese utterances contributed to the meaning of the discussions and sometimes facilitated the use of oral English.

\subsection{Unsuccessful Negotiation}

Negotiation in group work was not always successful. The unassisted students in four instances did not negotiate successfully. The unsuccessful negotiation of understanding occurred in the topic anger (two examples), and animals (one example). There was one unsuccessful negotiation of understanding in an assisted group for the topic advertisement. Following is an example of the unassisted group when the students were discussing the topic anger.

\section{Sequence 17: Unassisted Group: Topic - Anger}

$26 \mathrm{~S} 2$ If I am watching the TV program but it is cancelled by a sports program I will ask them to

27 S3 No, TV program. 
In the topic advertisement, the assisted students were advertising an exercise machine.

\section{Sequence 19: Assisted Group: Topic - Advertisement}

36 S2 In Vietnam there are no products to advertise ( $S 2$ was referring to the exercise machine). I think we should advertise another product.

$37 \mathrm{SS}$ In some books, magazine, you can see some bicycles. When we advertise these things, we can talk about a model. Maybe show a picture of a model. Do you know a model? A very nice woman. You can ask, "Do you want to be like her? How can you be like her?" And then we can talk about the product. I think it is rather interesting.

38 S3 Can we have a sample of the product?

39 SS How can we start our advertisement?

S2 suggested changing to another product but the senior student ignored S2's suggestion and kept on with other suggestions (36-37). S3 asked the senior student about a sample of the product that the senior student suggested. However, the senior student continued the talk about another aspect of the advertisement (38-39).

Although episodes of unsuccessful negotiation did not occur frequently, they showed different patterns of interaction in the two types of groups. The unassisted groups experienced more unsuccessful negotiation of understanding because peers sometimes ignored their partners' ideas. In addition, the students did not understand clearly the required tasks or their limited English proficiency prevented them from expressing their ideas. The failure in the assisted group was to due to the power relationship between the senior student and the group. The senior student was task-focused but in the example of sequence 19 , she tended to dominate the discussion and the student could not negotiate with her. 


\subsection{Discussion and Conclusion}

Sociocultural studies have tended to concentrate on that part of Vygotsky's work which discusses how thought arises from verbal communication during social interaction. They have generally studied the role of interaction in pairs and in groups. The results of the current study showed that language mediation occurred not only in the language the students were learning but also in their mother tongue. In addition, the patterns of mediation varied in the unassisted and assisted peer groups. The results from the audio transcriptions showed that mediation occurred in the process of negotiation of task planning and procedures and negotiation of understanding. During the group interactions, the students spoke to exchange thoughts, expand and adopt ideas as well as to promote thinking of the solutions to the questions from the textbook and from other peers. However, the process was not always successful. In some group discussions, utterances were sometimes ignored by other group members. The unassisted students experienced more negotiation of task planning and procedures but less negotiation of understanding. The senior student guided the students in the assisted groups, which helped them understand clearly what they had to do. As a result, the assisted students spent less time on task planning and did not use Vietnamese in group discussions. The unassisted groups used Vietnamese when a task was more cognitively challenging. They could also use Vietnamese at any stage of the discussion. The interactions in the unassisted groups were irregular in turn taking whereas in the assisted groups, the senior student controlled the interactions and spoke to individual members in the groups. The discourse of the students in both groups was unlike conversation in everyday settings. Behind the interactions, there was a textbook, a teacher, and set tasks designed to help the students to practise English. 


\section{Chapter 8}

\section{LEARNING VOCABULARY}

\subsection{Introduction}

Research in learning vocabulary in a second/foreign language is well documented (see for example, Nation, 1990). However, only a few studies have investigated learning vocabulary in group work (Knight, 1996; Nation, 2001; Newton, 2001). Nation (2001, p. 114) pointed out that, "With careful thought and planning, listening and speaking can be important means of vocabulary growth". Even when working with language tasks that do not require learners to learn a list of words, learners can learn words incidentally (Nation, 2001). Knight (1996) found that although learners used the first language a lot when working in groups, they were actually discussing unknown second language words. Code-mixing or the use of a first language word in a second language utterance was an effective and efficient way of learning a language (Celik, 2003). One of the challenging ways of learning vocabulary is to encourage learners to negotiate the meanings of new words among themselves, using each other as a resource rather than relying on external assistance (Newton, 2001) because while many words are not known by individuals, most are known by at least one other learner. Newton found that even without help from a dictionary or a teacher, so long as the learners were prepared to work cooperatively, they had sufficient resources within a group to tackle most unknown words. However, Newton did not point out what resources were used in a group. The resources could be the knowledge of new words in the target language, or the knowledge of concepts associated with words in the mother tongue for equivalent translation. The resources involve how students help peers with words. The students 
could also learn words from discovering the meanings of words from the written contexts provided in the textbook.

Sociocultural theory emphasises social interaction as a means of exchange of thoughts. This denotes an essential role of understanding word meanings. As viewed in this aspect, sociocultural theory can be used to explain the process of learning vocabulary in group work in a language classroom because it explains how learners use language to help each other to learn and social interaction brings new understanding of new words in learners. However, the literature of sociocultural theory does not document any studies that have explored the process of shared learning of word meanings in language classroom interaction.

This chapter answers the research sub-question, "How is vocabulary learnt when the students are practising oral English?" It presents negotiation of word meanings and forms. It is the process by which students shared their knowledge of word meanings and forms by helping to explain new words to each other. In the process of clarifying and explaining words, the students were co-constructing their understanding of concepts. The data sources for this chapter came from the audio transcriptions. Sequences at different stages of discussions were examined. Due to the dissimilar examples of negotiation of word meanings and forms in the two types of groups for each topic, the sequences of the unassisted peer groups are presented first and then those from the assisted peer groups. The data also came from the journal entries and interviews. The journal entries were examined and the three categories which emerged were: 1) the students provided examples of the words that they had learned, 2) joint learning of vocabulary in which the students shared their knowledge of words, and 3) 
collective memory in which group members through interaction helped other members in recalling and remembering words. Data from the interviews provided more details of the experience of learning vocabulary in the two kinds of groups.

\subsection{Audio Transcriptions}

Sequences from the topics: vacation, traditional and modern, taking risks, work, and good luck and bad luck, among many other sequences recorded, were chosen to examine the negotiation of word meanings and forms during group discussion. Sequences 20-23 were extracted from the audio transcriptions of the unassisted peer groups and sequences $24-26$ from the audio transcriptions of the assisted peer groups. The following sequence occurred near the end of the discussion of choosing a country for a vacation.

\section{Sequence 20: Unassisted Group: Topic - Vacation}

72 S4 What kind of holiday do you choose to go to Holland?

73 S2 I don't know what you mean.

74 S4 Kind of holiday means you go by yourself or you want to visit with any tourist company and they will take responsibility for all the activities you would do and journeys. They will choose the restaurants for you and decide which places for you to visit.

$75 \mathrm{~S} 2$ What about your idea?

76 S4 I think I would like to go to Holland on package holiday because I know nothing about Holland. So people in the tourist company will help me to see the most interesting Holland.

77 S3 They will guide about some sightseeing, where you want to go, the restaurants you want to eat.

S4, S2 and S3 were engaged in a collaborative negotiation of the meaning of the phrase kind of holiday. S4 was helping S2. S3 was listening and broke in to provide more details of kind of holiday. The recording ended at this point. It does not show whether 
$\mathrm{S} 2$ could use the phrase in the next utterances but line 75 seems to show that $\mathrm{S} 2$ understood the meaning of kind of holiday.

The students in the following sequence were to prepare two questions for each topic that they had studied.

\section{Sequence 21: Unassisted Group: Review Session}

$77 \mathrm{~S} 3$ Ban lam gi de phuc vu cho [What do you do to serve]. What can you do to contribute?

78 S4 Contribute la chi? [What does it mean 'contribute'?]

79 S2 Dong gop (Explains in Vietnamese the meaning of the word 'contribute')

80 S4 Contribute gi vay? [Contribute what?]

81 S3 Contribute to the success of the transport.

S4 was listening to S3 and asked for the meaning of the word contribute. S4 used both English and Vietnamese to ask. S2 broke in to help and S2 used Vietnamese to give the equivalent meaning of the word in Vietnamese (79). S4 then understood the meaning of the word and asked S3 to finish the early sentence that S4 interrupted (80). S3 then completed the sentence.

Sequences 22 and 23 below are examples of negotiation of word forms in the unassisted peer groups. In the following situation, the students were given a picture of a room to talk about, for example, what they thought about the room, who was most likely to live in the room, their age, taste of music, and so on. The textbook provided the questions and the classroom teacher provided the picture.

\section{Sequence 22: Unassisted Group: Topic - Traditional and Modern}

$88 \mathrm{~S} 1$ What do you like best?

89 S3 If you have a chance to choice something in this room? What will you choice?

90 S4 Choose. (12 seconds pause)

91 S3 I think I will choose this dish because I am now very hungry. 
S4 listened to S3 and offered correction for the wrong usage of the word choice. S3 accepted correction and used it in the new sentence correctly.

\section{Sequence 23: Unassisted Group: Topic - Becoming an Adult}

42 S5 What do you think about in Britain they are allowed to go to the pub and drink alcohol?

(Two seconds pause)

43 S3 I think drinking alcohol is very interesting.

44 S4 When you drink, you will be drank.

45 S2 Drunk.

46 S4 Drunk.

47 S2 Minh noi cai nay roi. Minh dang noi ve Britain. [We have discussed this. We are talking about Britain.]

S4 was responding to S3's idea. S2 corrected the word drank. S4 repeated and accepted the correction. S2 broke in again with another suggestion regarding the process of the discussion.

Sequences 24-26 below were extracted from the audio transcriptions of the assisted groups. In sequence 24 , the students were responding to a list of statements provided in the textbook. The situation was, "Someone pushes in front of you in a queue in a busy shop."

\section{Sequence 24: Assisted Group: Topic-Anger}

50 SS What about the third situation? Can you read it out?

$51 \mathrm{~S} 2$ Someone pushes in front of you in a queue in a busy shop (from textbook).

52 S4 Queue? What does it mean?

53 SS Whenever you buy something, you will have to stand in a row to wait for the other person to buy something and then it's your turn next. Suppose that you have to wait for your turn, you know, when you buy something, for example, the ticket. Do you understand?

$54 \mathrm{~S} 4$ Yes.

$55 \mathrm{SS}$ What will you do then? Will you get angry at the person?

$56 \mathrm{~S} 4$ Of course.

57 SS What else will you react?

58 S4 I think I talk with him about his behaviour. 
$\mathrm{S} 4$ was listening to the statement from the textbook read by S2. While S4 showed lack of understanding of the word queue, the senior student explained the meaning providing some examples (53) and checked to see whether S4 understood. S4 confirmed understanding and gave a response related to the situation (56 and 58). The concept of queuing is not very common in daily practice in Vietnam.

The students in sequence 25 were discussing the situation "Would you take part in a séance?" in the textbook:

\section{Sequence 25: Assisted Group: Topic - Taking risks}

33 SS Can we move to a new one? Would you take part in a séance? (from textbook) Do you understand the word?

34 S2 No.

$35 \mathrm{SS}$ Let me explain. A séance is a place where you can meet dead people. Something like superstitious.

36 S2 Dead?

37 SS Someone died. You know. For example, your grandparents died and you would meet them.

38 S2 I think I wouldn't because I am not a superstitious person.

39 SS So, you won't take part in a séance. In this place you can talk with a dead person.

40 S2 But I don't believe.

41 SS Ok, do you want to take part in this play?

42 S4 Yes.

The senior student suggested moving on to another situation. When she read out the word séance, she tried to find out whether the students knew the word (33). When S2 answered "no", the senior student gave an explanation (34). S2 was listening to the explanation and confirmed understanding by saying "Dead?" (36). The senior student provided further explanation to help S2 (37). S2 then understood the meaning of the word and provided an answer to the senior student's previous question (38). The senior student heard S2's answer and tried to elicit a further response (39). S2 gave a personal opinion and the senior student addressed another student (41). S4 who was listening to the conversation expressed a different point of view. The senior student and S2 were 
engaging in a process of negotiation for the meaning of the word séance and other members in the group appeared to learn from their interaction. Explanation by the senior student and checking for correct understanding by S2 made the process successful.

The students in sequence 26 were responding to a list of statements from the textbook. They had to decide whether a statement is related to a Japanese company or a European one.

\section{Sequence 26: Assisted Group: Topic - Work}

20 SS So you think that a Japanese company has a company song. Have you ever met this situation?

21 S1 I don't understand a company song.

22 SS For example, you work for a company and that company has a song for everybody to sing. Just the same song the song to think of the company. You know that. Do you think that it is European or Japanese?

$23 \mathrm{~S} 1 \mathrm{I}$ think both.

In Vietnam, there are no company songs for everyone to sing although some foreign companies in Vietnam may practise this tradition. The senior student explained to S1 what a company song was. S1 then understood the task and provided an answer to the question.

In the unassisted groups, there were four examples of negotiation of word meanings and forms in the review session and two examples in the topics good luck and bad luck, and becoming an adult. Other topics each produced one example except the topics anger, animals, jobs and salary, and taking risks. In the assisted groups, there were two samples of negotiation of word meanings in the topic good luck and bad luck and one example in other topics except the review session, and the topics urban and rural, traditional and modern, advertisement, and becoming an adult. In total, the unassisted 
groups produced 13 examples of this kind of negotiation as opposed to nine in the assisted groups. Among the 13 examples of the unassisted groups, six were about word meanings, five about word grammatical forms, and two were unsuccessful attempts. The nine examples of negotiation of the assisted groups were about word meanings. The transcriptions did not show any negotiation of word forms in the assisted groups. It could be that the students in the assisted groups did not make any mistakes with the word usage. Alternatively, the assisted students were cautious in their vocabulary usage when speaking in the groups with the senior student.

The negotiation of word meanings and forms embraces the process of exchanging and sharing understanding knowledge about words. The students negotiated the meanings of words more than the simple word forms. The sequences showed the collective effort in learning words in group work. The students in both groups were attentive to questions and the vocabulary use of group members. The students understood what words other members needed and provided prompt corrections and explanations. The unassisted students received help from the group members, but the pattern of negotiation of word meanings and forms in these groups was not predictable. Any student in the group might help other members. The pattern of negotiation of word meanings in the assisted groups was that the senior student explained the meanings of words if the students asked. She often checked for understanding. The students then were able to use the words in their utterances. The shared learning in the unassisted groups was collaborative whereas the process was expert-novice in the assisted groups. 


\subsection{Unsuccessful Negotiation of Word Meanings and Forms}

The negotiation of word meanings and forms was not always successful in group work. In the following sequence, S1 wanted to know the meaning of the word wink to use in her/his sentence. S3 provided winkle instead. However, this word was not correct in the context. S1 did not repeat the word but continued his/her sentence assuming the word was right.

\section{Sequence 27: Unassisted Group: Topic - Good Luck and Bad Luck}

20 S1 Nhay mat la chi? [What are the English words for 'wink eyes'?]

$21 \mathrm{~S} 3$ Winkle.

22 S5 Mat phai hay mat trai? [Right eye or left eye?]

$23 \mathrm{~S} 1$ You have good luck if your right eye nhay la chi rua? [What is the English word for 'wink'?]

24 S3 Winkle

25 S1 Right eye you will have bad luck.

S1 wanted to have an English equivalent of the phrase in line 20. S1 used Vietnamese. S3 provided the English equivalent. S1 in the episode above clearly considered the word provided by S3 was correct. There was no judgement by members of the group and the students worked on the system of trusting each other's knowledge. The Vietnamese utterances in lines 20 and 22 facilitated the expressing of the idea of S1 in English in line 25 although S1 still did not incorporate the word 'winkle'.

\section{Sequence 28: Unassisted Group: Topic - Good Luck and Bad Luck}

50 S1 Tha den hoa dang la gi? [What does it mean in English dropping small lanterns into the river?]

(Five seconds pause)

51 S2 Ho cung dau nam de mang lai may man phai khong? [Is that right that people make offerings to their ancestors at the beginning of a new year for good luck?] 
S1 wanted to express a Vietnamese idea but did not know the English equivalents and S1 asked for help with the words in Vietnamese. No one in the group answered. S2 broke in with another different idea. The concepts that the students were discussing were firmly associated with Vietnamese culture.

\subsection{The First Language in Learning English Vocabulary}

The negotiation of word meanings and forms happened more often in the unassisted groups than the assisted groups. The most common pattern of negotiation in the unassisted groups was that one person who wanted to know a word asked other students in the group in Vietnamese or in English and was supplied with an explanation in Vietnamese (sequences 21 and 27 this chapter). Another pattern was that they shared words in sentences or helped each other to correct the wrong use of words (sequences 22 and 23).

The unassisted students frequently used Vietnamese when they encountered new words. This indicated that the task required a level of English which was somewhat beyond the current level of the students. The first language was a firm source of help for the students. In the assisted group, the senior student was a fast and reliable help for explaining words or providing the words needed. The senior student used only English. However, this tended to prevent the contribution from other group members. 


\subsection{Journals}

The guiding questions in the journal did not ask a specific question about learning vocabulary. The students were asked if the discussion brought them new knowledge of English and how other group members or the senior student helped them in the group work. The data were sorted and the reports regarding learning vocabulary were put together. Seventeen students in the unassisted groups and 22 assisted students reported that the group work helped them learn new words. Six unassisted students and seven assisted students wrote that when learning in groups, they experienced joint-learning of vocabulary with other members in the groups. Interestingly, five students (one unassisted and four assisted) said that working with others helped them memorise vocabulary, a finding which could not have been established from the audio transcriptions. Some students also reported unsuccessful vocabulary learning (two unassisted students, and one assisted student). The students' reports on learning vocabulary are discussed in the following sections.

\subsubsection{New Words}

Although many students in both groups reported learning new words, not all of them provided the number of words that they learned. Following are some examples reported in the journals of both the unassisted and assisted students.

I learned some new words, such as funeral. (19-U-J)

I learned some new words such as pillow, carpet, and synonym of friendly. (34U-J)

New words through speaking with friends: pub and consent. (41-U-J)

Other group members explained the new word permanent. (12-A-J) 
Some new words about advertising an exercise machine. (39-A-J)

New words such as break law, and dismiss. The senior student explained some new words to me. (40-A-J)

The examples given above provide concrete evidence of learning new words. The data from the transcription showed that when the students encountered new words, they usually asked others. The data from the journal reported as presented in this section, however, indicated that some of them learned new words by listening to the interaction among the group members. One student reported, "Something I don't know, I can hear from other members" (44-J-U). Alternatively, the students could learn the words from the textbook. Some students recalled their experience of learning vocabulary in the group work:

Knew many vocabularies. (11-U-J)

Learned from other members sentence structures and new words. (32-U-J)

Learned new words through the ways of expression of other members in the group. (24-A-J)

New words used in advertisement. (29-A-J)

\subsubsection{Joint-learning of Vocabulary}

The students in the both types of groups also reported joint-learning of vocabulary. Here are some examples:

They helped me use more exact and effective words when I was expressing something. (23-U-J)

When I gave out an idea and I could not express some words, my friends helped me. (38-U-J)

I was helped by the senior student when I got some mistakes with the meaning from some words. (03-A-J) 
Other group members helped me with vocabulary so that I could express my opinions. The senior student helped us when we had problems with words, she provided suitable words. (23-A-J)

\subsubsection{Collective Memory in Learning Vocabulary}

Collective memory helped the students in the two kinds of groups recall and remember words. Some of the students' reports follow,

They helped me remember some words so that I could express my ideas. (25-U-J)

Friends helped remember low-frequency words in English such as celebrate and cage. (02-A-J)

When I forgot the meanings of new words, my friends could help me. (03-A-J)

For some words, I could not understand when listening to them, my friends explained to me and I found it easy to remember. (31-A-J)

Collective memory was used in different forms: students remembered the words they thought they had forgotten, or the interaction provoked their memory of the words. It also helped the students remember the words more easily. Remembering words in this sense requires the context in which the interaction took place, the use of the words by interlocutors, and attention from the interlocutors.

The students did not report learning word forms although the audio transcriptions of group work showed that the unassisted students helped each other with grammatical forms of words (for example, see sequences 22 and 23 in this chapter). However, two students wrote that other group members helped them with the pronunciation of words,

They helped me to pronounce some words correctly such as advertisement, and scholarship. (40-J-U) 
I say lives /livz/ but my friends helped me to pronounce /aivz/. (41-J-A)

When I was talking, my friends corrected my mistakes and I remember long those mistakes. (28-J-U)

\subsubsection{Report of Unsuccessful Learning Vocabulary}

The reports from the students' journals show some examples of unsuccessful vocabulary learning.

There were some new words that we did not know. (39-U-J)

The topic today was about business but our vocabulary for this field is not much and this was disadvantage. (40-U-J)

Student 40 reported that it was a disadvantage not knowing the vocabulary for the topic under discussion. Although only three students wrote about unsuccessful vocabulary learning, the audio transcriptions (sequences 27 and 28) showed that there were more instances and this could happen when a person asked for help but was ignored.

In summary, the answers from the journals indicated that the students, whether in the unassisted groups or assisted groups, did learn vocabulary. More assisted students reported learning vocabulary, 22 as opposed to 17 unassisted students, but the difference is small. In addition, the journals were based on students' recall and reflection, and not on a verbatim record. There were no reports from the senior student. The data from the journal entries support evidence from the audio transcriptions of the process of negotiation of word meanings and forms. Both the collaborative and expertnovice participation helped the students learn the words that they believed that they had not known before. More students from assisted groups reported learning new words 
and collective memory for words. They also referred to episodes which showed the operation of collective memory.

\subsection{Interviews}

In the interviews, I asked the students in Vietnamese, "How did your experience in the unassisted group and assisted group differ?" The interview question did not ask directly about learning vocabulary. However, as learning vocabulary was part of the process, the students gave some information on how they benefited or not from unassisted and assisted learning in terms of vocabulary. The students' responses reported in this section were translated from Vietnamese.

Two unassisted students reported that they did not know how to use words in group work.

Without a senior student, sometimes we could not use vocabulary. (20-IU)

When I met a new word that I did not know, I had to think for a long time. But when I worked with the senior student, I could ask her about the word. (05-IU)

These two statements indicate an important role played by the expert. Twenty assisted students reported their experience of learning vocabulary in the assisted group.

When I am speaking, if I forget words, the senior student can help me. (07-I)

The senior student helped me to express ideas and to use words correctly. (09-I)

She gave me suggestions so that I could say what I was supposed to. She helped me with vocabulary when I was finding my way. (14-I)

She encouraged us to speak more and I was able to learn more vocabulary from her. (24-I)

The students here again perceived the importance of vocabulary in the process of using English. They appreciated the role of the senior student in helping them with 
vocabulary. The data from the interviews matched the audio transcriptions which showed that the senior student explained new words to the students.

\subsection{Discussion and Conclusion}

The chapter presented evidence to show the ways in which the students learned vocabulary when they were practising oral English. The evidence was collected from the audio transcriptions, journals and interviews. The audio transcriptions provided data on the process of learning vocabulary. In the unassisted groups, the students used more Vietnamese in explaining new words. The assisted groups had direct help from the senior student who always used English to explain new words. Both the journal entries and interviews provided further details of what and how vocabulary was learned through group participation. Group members and the senior student appeared to have a mediational role in explaining new words, helping other students to understand word meanings and forms, and in being able to use new words. More students reported learning vocabulary in the assisted groups because they had an expert to help them.

The data from the journals and interviews provided examples of how the students learnt new words, used collective memory and received help from other group members in learning and using vocabulary. The interviews were more general but again confirmed the importance of learning vocabulary. The data from all sources appeared to show the advantage of working in groups for accessing someone who knew the required new words in English which conveyed the concepts and ideas needed for the discussions. However, there were instances when they misled each other. The students in the 
assisted groups had the advantage of working with the senior student who represented the expert.

As seen in the EFL approaches, the students were engaging with words and they also were using each other's vocabulary knowledge. In the process of social interaction they explored concepts, such as a séance, a package holiday, or a company song. They also shared knowledge of working styles in different countries and cultural practices, for example, queuing. The textbook in this study did not provide a word list for each activity. However, the thematic words written for these activities helped the learners expand their vocabulary knowledge. The students were not instructed to learn specific words but the data show that they learned words incidentally from discussion of the themes. Using Vietnamese helped the students to code-mix their English utterances with Vietnamese words to express their ideas. 


\section{Chapter 9}

\section{ARTIFACT USE}

\subsection{Introduction}

In sociocultural theory, artifacts also play an important role in the mediating process. However, as mentioned in section 3.11, Chapter 3, classroom research using a sociocultural theoretical framework focuses mainly on the mediational role of oral language. Artifacts include both physical objects and immaterial cultural tools (Wertsch, 1998). In Vietnam, where English is not used as a native language, learning the language depends on situations presented primarily in textbooks. The students in this research used the textbook Well Spoken (see section 4.7, Chapter 4).

This chapter is an attempt to answer the research sub-question, "What artifacts function as mediational tools?" It reports how the textbook, blackboard instructions, and classroom handouts were used by students in the two different kinds of groups. Furthermore, it discusses the role of cultural understanding as mental artifacts. Culture is constituted by artifacts which include both concrete objects and mental artifacts, such as cultural schemas, representations and the like (section 3.7, Chapter 3). This chapter provides evidence of how the students reproduced their internalised cultural representations in response to the textbook questions and other artifacts. The data sources for this chapter came from the audio transcriptions and the video tapes. The chapter ends with discussion and comparison of how artifacts were used in the two group settings. 


\subsection{Audio Transcriptions}

The pauses from the audio transcriptions showed a significant difference between the unassisted and assisted groups, 707 seconds of silence in the unassisted groups compared to 245 seconds of silence in the assisted groups. It was possible that the unassisted students took more time to think, to form ideas and utterances in English, or to respond to other members in the groups. They could take time to write down their ideas as, for example, revealed in the audio tapes of the review session, when the students were asked to prepare questions for each topic they had studied. The pauses also seemed to indicate that the students in both types of groups were referring to the artifacts. As seen from the audio transcriptions, in the assisted groups, the senior student's task-focused questions engaged the students more in interaction (for some examples, see the sequences 11,13 and 15, Chapter 7). Her utterances could have taken places of the pauses in the unassisted groups. As a result, there were fewer intervals of silence on the audio tapes of the assisted groups.

In 9 of the 12 topics for discussion, the students were required to answer questions related to topics in the textbook. In the following section, the role of the textbook as an artifact is discussed.

\subsubsection{The Textbook}

The textbook used is an artifact which stores some kind of knowledge, generally in written form, and provides the author's suggestions for learning and teaching. The audio transcriptions showed that in the nine topics with the textbook questions, the 
discourse structure was similar and so was the pattern of use of artifacts. A student in an unassisted group read out a question in the textbook and other group members joined in the discussion (see sequences 10,16 and 18 in Chapter 7). In the assisted groups, the senior student always told the students which part in the textbook they had to view and what the required tasks were. The students working with the textbook discussed a range of specified topics, including, for example, work. The following two sequences of the audio transcription of the topic work illustrate how the textbook was used. The task in sequence 29 is, "Different countries have different styles of working. For example, Japanese companies are very different from those in Europe. Do you think these statements are more likely to be made about Japanese or European companies?" The textbook provides a list of statements.

\section{Sequence 29: Unassisted Group: Topic - Work}

$01 \mathrm{~S} 1$ People expect to work for the same company all their lives (from textbook). Do you think this is about Japanese or European companies?

02 S2 I think Japanese.

03 S1 Why? (10 seconds pause)

04 S2 Because Asian people think relationship with other people is important so they expect to work for the same company all their life.

05 S3 I agree because if they work for the same company all their lives, their salary will be increased. (Three seconds pause)

06 S4 Maybe both.

07 S1 Cau hai hi. [Let's move to question two.] (Three seconds pause)

08 S5 There is a company song which everyone sings (from textbook).

S1 read from the textbook. S2 responded. The situation presented in the textbook generated two different points of view from S2 and S3. S2 brought in her knowledge about Asian working styles and S4 agreed with S2 and S3's answers. The textbook determined the procedure and the ways the students used their prior knowledge to respond. 
Here, similarly to sequence 29 , the students were responding to the textbook question:

\section{Sequence 30: Assisted Group: Topic - Work}

01 SS Are you ready? Part one, OK. So you should start to choose which of these are more likely to be made about Japanese or European companies. What do you think? Who made this statement? (The students were reading statement one in the textbook.)

02 S1 I think Japanese.

03 SS Do all of you agree?

04 All Yes.

05 SS What do you think?

06 S2 People expect to work in the same company all their lives because they got good jobs. ( $S 2$ was reading from the textbook and added some contribution.)

07 SS It's good. So this does not mean Japanese.

$08 \mathrm{~S} 1 \mathrm{I}$ think in general Asian not only Japanese people do not want to change their jobs in their lives.

09 SS That sounds good. That means they started with fixed jobs. How about the next one?

What the senior student said was based on the textbook. She pointed out which part the textbook the students had to read. She asked a group member to read part of the text. The senior student used the textbook as a guide for further discussion. The students used the textbook for information.

As seen from the two sequences 29 and 30, the students in the two kinds of groups approached the task in a slightly different way. The unassisted students were working directly with the textbook. Whereas, in the assisted group, while the senior student assumed the role of a teacher, what she told the students was based on the textbook. Ultimately, therefore, the content and direction of the discussions of the two groups were determined by the textbook. The textbook provided the general background for the activity and determined the procedure and order of students' discussion. The situations in the textbook stimulated students' thinking and generated ideas for the 
discussions. The silence on the audiotapes will be explained in the discussion of the video data (see section 9.3).

The four following sequences illustrate how the textbook questions determined the structure of group discussion. In sequence 31, the students were discussing whether certain animals should be used for eating, working or kept as pets. The textbook provided a list of animals.

\section{Sequence 31: Unassisted Group: Topic - Animals}

54 S5 Do you like a fox, Hoa?

55 S3 No. I haven't seen it. I saw it on TV. It is a dangerous animal.

$56 \mathrm{~S} 1$ Because in many fairy stories, it is considered as a dangerous and cruel animal. I think it is a wild animal and must be protected.

57 S4 Do you think fox is very intelligent?

58 S3 Smart?

$59 \mathrm{~S} 2$ Yes.

$60 \mathrm{~S} 1$ How about a duck?

61 S3 Very popular meat! (Laughter) (End of tape)

As the textbook had asked, the students discussed each animal in turn. Having talked about a fox, they went on to discuss a duck (60). The students laughed because of the familiar image generated by lines 60 and 61 in the culture that they knew.

A sequence of an assisted group relating to the same topic follows:

\section{Sequence 32: Assisted Group: Topic - Animals}

92 SS How about a fox? You know this one? Do you think a fox can be kept as a pet?

93 S3 No.

94 SS Is it a dangerous or a nice animal?

95 S3 Dangerous.

96 SS Do you think we can eat a fox?

97 S4 No, just treat as wild animal.

98 SS Have you ever seen a fox used as a working animal?

99 S4 No.

$100 \mathrm{~S} 1 \mathrm{It}$ can be used in a film. In some kinds of film, people use a fox to play some roles. 
101 SS So it can be used as a working animal.

102 S1 Yes, I think so.

103 SS How about a duck? Do we have ducks in Vietnam?

104 Sl Yes.

(Laughter)

As with the unassisted group in sequence 31 , the students in the assisted group went from talking about a fox to talking about a duck. All students whether in unassisted and assisted groups laughed at familiar cultural image of a duck. The textbook elicited knowledge from the students in a form of what they had seen from film or from daily observation.

Following are two more illustrations of how the textbook determined the continuity of the discussions.

\section{Sequence 33: Unassisted Group: Topic - Urban and Rural}

44 S5 I think in the country people were not fashionable because of their working conditions.

45 S1 Holidays.

$46 \mathrm{~S} 4$ Holidays is a public time for people to relax. In the city there are entertainment activities. People can go swimming and dancing.

The students were discussing the clothes that people in the country wear before going on to another situation. The textbook then guided them to discuss holidays.

\section{Sequence 34: Assisted Group: Topic - Urban and Rural}

29 SS In brief, do you think that your health is better in the city or in the country?

30 All In the country.

31 SS How about holidays? We should move to the next one.

32 S2 I think holidays in the country are more interesting because in the country there are more beautiful places. I like green fields.

Here, the list of topics and questions in the textbook determined the order in which they were discussed. 
In summary, the textbook provided directions for the students to follow. During group work, the students read out the situations provided in the textbook and referred to the textbook for instructions about the task. However, the textbook was used differently in the different types of groups. The unassisted students made more use of the textbook, whereas the assisted students followed the senior student who was herself guided by the textbook. Regardless of whether they were in groups of peers all at the same level or groups of peers and the senior student, the students were dependent on the textbook to guide them. The textbook offered both "affordances" and "constraints" to the students' discussions. On the one hand, it helped generate ideas. On the other hand it structured and limited the contents and directions of the discussions.

\subsubsection{Other Classroom Artifacts}

In the three topics vacation, review, and advertisement, the students did not have detailed questions from the textbook to respond to. In discussing the topic vacation, the students used travel brochures given to them by the classroom teacher in order to discuss an imagined vacation. The brochures contained relevant information and the senior student told students where to look, "Please tell me which picture you want the first, the second or the third? You have to choose three countries, three cities, three activities, and three restaurants. Which place do you want to go? You can choose from this magazine". The transcription of the unassisted group did not reveal how the students consulted the brochure.

In the review session, the senior student asked group members to refer to the textbook, "OK, are you ready? Can you tell me what units you have already done? Can you tick 
the units you have already done?" The transcription of the unassisted group did not show that the students referred to the textbook for the essential information (see sequence 04, Chapter 7). In the topic advertisement, one unassisted student wanted to sell a pen, while the assisted group chose clothes. When the senior student suggested choosing an exercise machine, the assisted group seemed to be confused because they perhaps had never seen one.

In the topic traditional and modern, the students used both the textbook and the pictures in doing the task. The pictures mediated the ideas of the students in relation to the questions in the textbook. For example, a student in an unassisted group stated, "I think the room is for an old man because there are a pair of glasses" (audio transcription of the topic traditional and modern). This is an example of reasoning or what Vygotsky (1978) called "higher mental function". An assisted student also made an assumption when looking at a picture from the classroom handout.

69 SS Who lives in this room?

70 S2 A child?

71 SS Why?

72 S2 Because the room is very small.

(Transcription of the topic traditional and modern.)

Unlike the textbook, a travel brochure, a pen, clothes and an exercise machine did not determine the structure of the discourse. The objects, whether concrete or imagined, provided illustrations which served to mediate thoughts among the students. For example, the brochures made the viewers want to travel to a certain country, and a picture of a room generated thoughts about the persons who lived in it. In the review session, the textbook served as a store of relevant information. 


\subsection{Video Recordings}

I viewed and reviewed the videos. I then wrote down the descriptions of what was happening during the group work. The videos provided images of the use of artifacts, or references to them. Often in the pauses noted in the audio transcriptions, the students were working with artifacts. The teacher wrote instructions on the blackboard and they were consulted by the students when they wanted to know what they were required to do.

The most commonly used artifact was the textbook. The students in the two kinds of groups referred to the textbook at all stages of a discussion. They also looked at the instructions on the blackboard. They used paper, notebooks and pens for writing. When the groups were discussing a vacation, they looked at the brochures that the classroom teacher gave them for information. In the topic traditional and modern, the students were looking at the pictures of the rooms and houses that the teacher gave. In the topic advertisement, the unassisted group used a pen as an object to make an advertisement. In one instance, a student from the unassisted group, when looking for examples of good luck and bad luck, turned and searched through her notebook. This incident could not be noticed from the audio transcriptions. The student used the notebook as a resource for keeping and searching for information. One student also used a dictionary to check the meaning of the word "funeral".

A major difference in artifact use in the two kinds of groups was in the frequency of reading blackboard instructions. The unassisted groups produced 14 instances (four instances in groups, three in pairs, and seven individually) of looking at the blackboard 
for information, whereas the assisted groups referred to the blackboard two times individually and one time as a group. The instructions on the blackboard were, therefore, more important for the unassisted students as they did not have the help of the senior student.

Individuals in the unassisted groups consulted the textbook less often than the individuals in the assisted groups (three incidents in the unassisted groups as opposed to 14 incidents in the assisted groups). The reason was that when the senior student was talking to the students, individuals in the assisted groups were both listening to her and reading along with her. However, the students of both kinds of groups read the textbook in groups quite often ( 20 incidents for the unassisted groups and 21 incidents for the assisted groups). The unassisted groups wrote more often than the assisted groups, 18 incidents as opposed to 10 incidents for each group respectively.

Travel brochures and pictures of rooms and houses were consulted 11 times for the unassisted groups and 9 times for the assisted groups.

\subsection{Artifact Use in Other Classes}

In the interviews, I asked the students about the learning aids used in other class sessions beside the class under observation in this research. All of the students reported the main use of textbooks. They also reported the use of cassettes in the listening classes which the students attended separately from the speaking classes. 


\subsection{Vietnamese Culture as Mental Artifacts}

The following sequences illustrate how Vietnamese cultural understanding served as mental artifacts, which mediated the students' responses to the questions in the textbook. The students reproduced the common behaviours and practices in Vietnam. In the topic anger, the instruction from the textbook asked the students to respond to the situation, "Someone bumps into your car when you are waiting at the traffic lights". Two students in an unassisted group stated:

$16 \mathrm{~S} 1$ Someone bumps into your car when you are waiting at the traffic lights, what will you do, $\mathrm{Ha}$ ?

17 S2 I think the same one hits your motorbike.

In Vietnam, especially in the city where this research took place people mainly travel by motorbike. S2 thought it would be more suitable to talk about the situation with a motorbike rather than a car. S2 was clearly referring to the knowledge of cultural norms controlling behaviour: cars and motorbikes and their use in daily life. S2 was referring to the means of transport that S2 was familiar with.

For the same topic anger, the students in an assisted group were discussing the situation, "Your child runs across the road without looking at the traffic."

42 SS So usually the father in the family gets angry and beats the child if the child does wrong things. Will you beat your child?

$43 \mathrm{~S} 3$ Yes.

44 SS As a mother, will you do so?

45 S2 Maybe I will beat.

In response to the situation, the students were referring to the Vietnamese cultural practices of physical punishment of children. Physical punishment from parents to 
children in Vietnam is accepted. In Vietnamese culture, the tradition is practised as this saying means, "Spare the rod, and spoil the child."

For the topic rural and urban, the unassisted students were discussing the differences between people in the country and in the city in their holiday time.

46 S4 In the city there are entertainment activities. People can go swimming and dancing.

47 S2 Children in the country can make kites and fly kites. They can play riding buffalos, can go fishing, hunt some birds, or can catch some fish.

The textbook probably aimed to ask students from different cultures to talk about universal practices of people on holiday in the country and the city. S2 however referred to the cultural practices that S2 knew. The knowledge of Vietnamese culture was in S2's mind.

The following excerpt from the assisted group illustrates a further reference to Vietnamese culture in an assisted group. The students were talking about the belief of good luck and bad luck in Vietnam.

70 SS The first one to enter your house to visit your house on the first day of a new year is a woman. Do you think that it is good luck or bad luck?

$71 \mathrm{~S} 3$ I don't know.

72 SS How about you? Do you think it is bad luck?

$73 \mathrm{~S} 4$ It depends on the person. If the person has good relation with your family, you will have good luck. But if the person has bad relation with your family, you will have bad luck.

As mentioned before, the association between good relationships with others and good or bad luck is a belief commonly accepted in Vietnam. What the students knew mediated what they said. 
The same idea was expressed during the topic work when an unassisted student said, "Asian people think relationship with other people is important so they expect to work for the same company all their lives."

There were no correct answers to the questions in the textbook. In the topic jobs and salary, the students in both an unassisted and an assisted group considered teachers to have the highest prestige. In Vietnamese culture, the value system is expressed as, "First emperor, second teacher". The students used this value as a standard for their response to the textbook question.

\subsection{Discussion and Conclusion}

This chapter reported how artifacts were used in the two group settings. Artifacts include both physical artifacts and mental ones. In examining the physical artifacts, the chapter focused on the textbook, classroom handouts, learning aids, and instructions on the blackboard. In examining the mental artifacts, the chapter collected data on how the students' first cultural understanding as mental artifact was reproduced in their discussions, and how these mediated the students' ideas.

The results showed that the textbook determined the structures of the discussions. It gave instructions to the students about what to do. The textbook controlled the continuity of the group discussions because the changing of questions was dependent on the topics or the sub-topics designed in the textbook, whether the students were in the unassisted or assisted groups. 
In Vietnam, access to English materials for classroom studies is limited. It is clear from the data presented in the chapter that the textbook was the main source for the tasks observed. Textbooks are also the main source for teachers' lesson planning in Vietnam (Lewis, 1996). Textbooks or other artifacts are physical tools, but they contribute to the use of the psychological tool, or the language of both Vietnamese and English. The textbook represents a written artifact. In this case, written language also has mediational roles in generating oral language.

The videos provided images of how the students used the artifacts in the ways that could not be heard from the audio transcriptions. The students looked at the textbook at any stage of a discussion. They read instructions on the blackboard and used other classroom handouts to respond to the questions of the assigned tasks.

The audio transcriptions also showed evidence of how the students, whether in the unassisted or assisted groups, made use of their knowledge of Vietnamese culture, of the textbook, and of other students in the process of knowledge construction in learning English as a foreign language. Classroom artifacts and cultural beliefs and values generated ideas from the students. The students referred, for example, to their familiar objects and the use of a motorbike and a kite, and the value of the high prestige of teaching.

In summary, the textbook was used as a main source of information in the discussions of both types of groups. It guided the discussions and mediated the students' responses. Other classroom artifacts worked as resources for the discussions. The students in the unassisted groups read the instructions on the blackboard more often than the students 
in the assisted groups. Individuals in the unassisted groups wrote on sheets of papers and took notes more often than individuals in the assisted groups. 


\section{Chapter 10}

\section{STUDENTS' FEELINGS}

\subsection{Introduction}

This chapter addresses the research sub-question, "Do students have different feelings according to the kind of group they are in?" As discussed in section 3.9, Chapter 3, Vygotsky and other sociocultural theorists (for example, Lantolf, 2000a; 2000b, Mercer, 2001, Sfard \& Kieran, 2001) have focused more on cognitive aspects of the process of learning, leaving the affective side relatively unexplored. A few other sociocultural theorists, for example, John-Steiner (2000), and Mahn and John-Steiner (2002), however, claimed that emotional experiences play an important role in helping children and adults develop. Human beings do not possess only cognitive functions; emotions are part of the learning process. The Vietnamese students in the current study were learning English as a foreign language. Apart from the feelings aroused in interaction with peers, they may also experience certain feelings when speaking another language. The feelings may be of enjoyment and/or of stress or be neutral. The feelings may be bad or good about one's performance. It is thus worthwhile to explore two emotional states involved in the process of learning EFL in the classroom: enjoyment and stress.

\subsection{Laughter}

Incidents of laughter were heard on the audio tapes: 1) The students laughed at the ideas related to the task (13 incidents in the unassisted groups and 10 in the assisted 
ones); 2) The students laughed at each other (18 incidents in the unassisted groups as opposed to eight incidents in the assisted groups); 3) The students laughed when discussing some Vietnamese practices related to food, and belief in good luck and bad luck (12 incidents for the unassisted groups as opposed to 10 for the assisted groups); 4) The students made jokes which were not directly related to the task (eight instances in the unassisted groups); and 5) The students responded to humorous comments by the senior student by laughing. The topics which produced the most laughter were advertising, taking risks, and traditional and modern. In the following section, sequences that contained episodes of general laughter among group members are presented.

\subsubsection{Laughing at the Ideas Related to the Tasks}

\section{Sequence 35: Unassisted Group: Topic - Anger}

$36 \mathrm{~S} 1$ Someone leans out of your car (from the textbook).

37 S2 Lean (pronounces the word again)

38 S1 And makes a rude sign at me, shouting that I am a bad driver (from the textbook).

39 S5 I think he is impolite.

40 S1 Impolite.

41 S5 Do you think he is mad?

(Laughter)

The students in the following sequence were required to advertise an exercise machine.

\section{Sequence 36: Assisted Group: Topic - Advertisement}

65 SS Imagine some easy name of the product. Some name easy to remember. It must be easy to call.

66 S4 "Perfect".

(Laughter) 


\subsubsection{Students' laughing at Each Other}

The students were creating an advertisement for a pen.

\section{Sequence 37: Unassisted Group: Topic - Advertisement}

71 S2 I can use it for five months.

72 S3 Really?

73 S2 Yes. You can believe me.

74 S1 I don't believe. Maybe I shouldn't believe you. (Laughter)

The following sequence is an example of students' laughing at each other in the assisted group.

\section{Sequence 38: Assisted Group: Topic - Urban and rural}

16 SS So you think you have good health in the city because you do exercise? In the country or in the city?

17 S4 In the city.

$18 \mathrm{~S} 1$ Tell a lie.

(Laughter)

\subsubsection{Laughing at Vietnamese Cultural Beliefs and Practices}

Sequences 39 and 40 show a similar pattern of students' laughing at familiar Vietnamese cultural practices. The students were talking about the belief of good luck and bad luck.

\section{Sequence 39: Unassisted Group: Topic - Good Luck and Bad Luck}

$27 \mathrm{~S} 1$ Before you take an examination, if you eat banana, and eat egg

$28 \mathrm{~S} 3$ and bean

29 S5 If you eat bean you will pass the exam.

(Laughter)

30 S1 Before examination, if you eat egg, you will have bad luck.

(Laughter) 
Sequence 40: Assisted Group: Topic - Good Luck and Bad Luck

06 S2 When we go out of home, if we see a pregnant woman, we will have bad luck.

(Laughter)

07 SS Oh, do you think so?

08 S2 Yes, my mother tells me.

\subsubsection{Laughing Because of the Senior Student's Humorous Remarks}

The students were talking about activities for a vacation.

\section{Sequence 41: Assisted Group: Topic - Vacation}

S2 I want to go book shopping.

SS What do you mean? What do people sell there?

S2 Book shop to buy some books.

SS Industrious person.

(Laughter)

The students were talking about the willingness to take risks.

Sequence 42: Assisted Group: Topic - Taking Risks

24 S4 Would you give up your job without having a new one?

25 S3 Maybe I would. In case the job is not suitable for me.

26 SS But you haven't got a new one. And you stay at home.

27 S3 Yes.

28 SS Very daring.

(Laughter)

\subsubsection{Off-Task Laughter}

The students were talking about what people can do at certain ages in Britain.

Sequence 43: Unassisted Group: Topic: Becoming an Adult

47 S2 Minh noi cai nay roi. Minh dang noi ve Britain. [We have discussed this. We are talking about Britain.]

48 S3 Noi tu nhien di. Mien noi tieng Anh la duoc. [Speak naturally. Speak whatever we want but in English.] 
49 S1 Now do you want to get married?

$50 \mathrm{~S} 3$ Yes.

(Laughter)

51 S1 I want to get married but I can't.

52 S5 You haven't got enough age.

$53 \mathrm{~S} 2$ Over 25 for men in Vietnam to get married. Over 22 for women.

$54 \mathrm{~S} 1$ But you can wait.

(Laughter)

55 S3 I don't want to wait. I want to get my wife.

(Laughter)

The students were not asked to discuss their own marriage. All the students in the current research were single.

As seen from the sequences in this section, laughter was generated while the students were working in either peer groups or peer groups with the senior student. Laughter occurred after the students had used English. The students seemed to enjoy playing with the language they were learning. The exchanges often appeared to be fun for the students. Social interaction and negotiation of understanding involve feelings. The sequences in this section suggested that peers in a group, or an activity itself (in this particular study, a language task) could arouse laughter.

\subsection{Enjoyment}

Most of the responses presented in this section came from the students' answers to the journal question, "Did you enjoy the group work today? If yes, what in particular did you enjoy?" (See Appendices F and G). However, responses from all journal reports relevant to the issue of enjoyment were put together. When asked about enjoyment, 41 unassisted students and 43 assisted students answered "Yes, I enjoyed the group work". Two students in the unassisted groups reported that they enjoyed group work but not much. Four students, two from each kind of group, reported that they did not enjoy the 
group work. With such a high number of students giving positive responses to the enjoyment of group work, it was desirable to check if they did so to please the researcher. Therefore, in the following section, quotations that the students provided about their enjoyment are presented and triangulated with the data presented in Chapters 5, 6, 7 and 8 .

Whether in unassisted or assisted groups, the students said that they enjoyed group work. What most of them enjoyed was working with friends (32 and 28 responses from the students in unassisted and assisted groups respectively). Twelve assisted students but only three unassisted students reported that they enjoyed the discussion because they had opportunities to speak English. Several students reported that they liked the topics (four responses in the unassisted groups and six responses in the assisted groups). Ten assisted students enjoyed the discussion because they had assistance from the senior student. One unassisted student stated that he/she enjoyed the discussion because it helped to prepare for the exam. Some comments follow:

In the group work today you can give your ideas what you think about that problem. You also can learn a lot of good ideas from members in group. (04-U-J)

I really liked the discussion today because there were a lot of ideas from other friends which helped me very much. (01-U-J) [Translation from Vietnamese]

Yes. I enjoyed the group work today because I could express my thoughts to my friends. Besides, I could get more information from my friends' ideas. (14-U-J)

I enjoyed the whole group when each of us tried to discuss the topic. (03-A-J)

I enjoyed the enthusiastic contribution of ideas from me and my friends. (20-AJ) [Translation from Vietnamese]

I could gain ideas from friends and the senior student about advertisements. (29-A-J) [Translation from Vietnamese] 
The enjoyment, according to the students who reported, stemmed from the exchange of ideas with the group members who were addressed as friends. In addition to this kind of enjoyment, the assisted students considered encouragement and guidance from the senior student made them enjoy their group work:

Yes, with a senior student, every member in the group felt less stressful when answering questions or speaking. The English level of the first year students was not sufficient to express what we wanted to say, so with assistance from the senior student, we could speak better. (02-A-J) [Translation from Vietnamese]

I liked the group work today because there was assistance from the senior student so I could speak more about this very interesting topic. (10-A-J) [Translation from Vietnamese]

Assistance seemed to bring more confidence to participate in the discussion.

The topic was one of the other factors that led to enjoyment. The students' responses regarding the topics somewhat overlapped with the responses presented in section 5.3.4, Chapter 5. However, the students' responses presented here were associated with positive feelings:

Yes, I enjoyed the group discussion today because talking about health and people in the country and in the city was the most interesting. (27-U-J) [Translation from Vietnamese]

I liked the group discussion today because the topic is easy to speak and I can present my opinions. (30-U-J)

I enjoyed the group work today. The thing I liked best is I could have a chance to talk about my vacation in the future. (04-A-J)

I loved speaking English and discussing with friends about the topic work. (37A-J)

Some reports of some unassisted students were conventional rather than personal reflection. 
Group discussion has some advantages because it helps me to have opportunities to say more and understand the lesson better. (45-U-J) [Translation from Vietnamese]

I enjoyed the group discussion today because we were assisted by the senior student and had more chance to speak English. (45-A-J) [Translation from Vietnamese]

Following are some examples from the journals explaining why the students did not enjoy the group work:

No, not much because I am not a superstitious person so today discussion was ok. (19-U-J)

Sometimes, group members' ideas were in conflict so the discussion was not interesting. (38-U-J) [Translation from Vietnamese]

The students' reports presented in the chapter provided more information on the students' experience in the group work. The data support what was presented in Chapters 5 and 6, which showed that the students found some topics interesting to discuss and that the assisted students used more English.

\subsection{Group Members' enjoyment}

In the journals, the students were asked, "Do you think that other group members did not enjoy the discussion today? If so, can you explain why they did not like the group work?" Thirty unassisted and 36 assisted students reported that they thought other group members enjoyed the group discussion. Twelve unassisted and five assisted students did not think that other group members enjoyed the discussions. Seven students (three in the assisted groups and four in the assisted groups) left their answers blank. It could be that these students did not want to write about other students' feelings or they did not pay attention to the journal question. 
Thirty students in the unassisted group said that their friends enjoyed the group work. Following are some extracts:

I don't think that some students did not enjoy the group work today because they all attended the discussion enthusiastically. (05-U-J)

I think everyone in my group liked the group work today. The discussion introduced interesting things and we as friends could understand each other better. (22-U-J) [Translation from Vietnamese]

No, I don't think so. I realise the enjoyable feelings on their faces. (26-U-J)

These students reported the group interaction which involved the contribution of other members and the relationships among the group members.

Thirty-six assisted students reported active participation and exciting discussion. Some of the assisted students said that they enjoyed working together. They also wrote about the eagerness of group members to participate in the group work.

Each member spoke a lot and it is an interesting discussion. Especially there is the senior student. (04-A-J)

I think that every student in my group enjoyed the group work today because I find that we discussed excitedly. (05-A-J)

I think that they were satisfied because we had an interesting conversation. (09A-J)

The students reported both positive and negative feelings associated with the group work. Twelve students in the unassisted groups gave examples of why some other students in the groups did not enjoy the discussion. The reasons as reported in the journals included shyness and stress (five responses), feeling of inferiority and worrying about making mistakes when speaking to friends (two responses), working with new group members (one response), lack of motivation to learn (one response), no assistance from a senior student (one response), different ideas of different members 
(one response), and being unable to explain why (one response). Following are some examples:

One or two students did not enjoy the group work today because they were shy or their knowledge of English is poor or they do not have enough vocabulary to speak English fluently. (12-U-J)

Some members did not enjoy the group work today. It could be that they had a feeling of inferiority or the topic was not interesting. (23-U-J) [Translation from Vietnamese]

The reports based on students' observations suggested that the group discussion was not always enjoyable.

Five assisted students reported negative emotional factors amongst group members.

Some students did not enjoy the group work because they were shy and worried about things that they could not tell to someone. (03-A-J)

Only one person did not enjoy the group work. He may be shy and afraid of discussion in the group. (06-A-J)

The answers seem to show that lack of participation was interpreted by students as lack of enjoyment.

\subsection{Stress}

Emotional reactions include not only enjoyment but also stress. In the journals, the students were asked, "Did you find the group work today stressful? If yes, please explain. If no, why not?" Eleven unassisted and eight assisted students said that they experienced stress during the group work. Thirty-four unassisted and thirty-six assisted students reported that they did not experience stress. One assisted student left the 
answer blank. The stress, according to two of them, was from the experience of being recorded (see section 4.4, Chapter 4).

In the unassisted groups, five students reported that they felt stressed because they had to concentrate on listening to other members, and were required to express ideas. Being afraid of making mistakes when speaking English was reported as causing stress by two students. These two students said that they felt stressed in the first few minutes and after that they gained confidence to continue. Thinking of a place for a vacation caused stress in one student who could not think of anywhere to go. Another student reported stress because she was shy. Some of the students' journal reports are as follows:

I felt stressed because I had to speak English from beginning to end so I felt difficult to express what I needed to speak in English. (13-U-J)

Yes, I felt stressed because I had to concentrate to keep up with friends and I had to contribute my ideas. And the ideas must be correct. (20-U-J) [Translation from Vietnamese]

In the assisted groups, eight students reported stress. Like some unassisted students, they felt stressed for the first few minutes but after that they participated in the group work. Lack of vocabulary caused stress in two students. Two assisted students said that group work sometimes made them work harder and this led to stress. The task of making an advertisement made one student feel uncomfortable, as it was unfamiliar and his/her vocabulary was insufficient to discuss the topic. Working with the senior student caused stress for one student because he/she was afraid of not speaking English well with the senior student.

Yes, I felt stressed a little because the topic today was difficult and my vocabulary for the topic was limited. (34-A-J)

Maybe I feel stressful today because I speak with the senior student. I was afraid that I could not speak well. (25-A-J) 
Some examples of the students' responses for not feeling stressed are as follows:

No, I didn't. I absolutely felt comfortable because it helped me to improve my speaking and my English knowledge. (16-U-J)

No, absolutely not. On the contrary I felt comfortable and excited. My friends had special and humorous ideas so the atmosphere was friendly and cheerful for learning. (32-U-J) [Translation from Vietnamese]

I did not find group work stressful because many behaviours of my friends made us laugh a lot and the detail we discussed today was very interesting. (08-A-J)

No, not at all. On the contrary, I felt comfortable and excited because it was a good opportunity to express our ideas in English. With the assistance of the senior student, I felt more confident when I spoke. (32-A-J) [Translation from Vietnamese]

One student reported that learning with an open textbook brought a comfortable feeling rather than stress:

No, I did not feel stressed because it was a review session so we could open the course book and look at it. (18-A-J) [Translation from Vietnamese]

In both types of groups, some students reported that they did not experience stress. Instead, they reported enjoyment. The reasons that the students provided in response to the question about stress also support the reliability of their answers. The students may have enjoyed the group work and felt stressed in the same session. However, none of the students reported this feeling. Nor did they give contradictory reports.

\subsection{Emotions and Learning}

As pointed out in the introduction of this chapter, sociocultural theory has generally overlooked emotional factors in favour of cognition. The reports from the students in the current study suggested how feelings and learning were interrelated in the process of group work. In the journals, the unassisted students reported that they enjoyed the group work because they learned new ideas from group members (eight responses) and 
shared ideas with group members (five responses). Some considered that the group work improved their language skills, for example, speaking and listening skills (three responses), and through group work, they could correct their language mistakes (two responses). One unassisted student reported that he/she enjoyed learning the things that he/she did not know by listening to other members (44-U-J). In the assisted groups, the students similarly reported that they enjoyed the group work because they learned new ideas (nine responses), improved language skills (four responses), and shared ideas (two responses). In addition, four assisted students reported they enjoyed learning new ideas as well as language skills from the senior student. Some comments follow:

I learned from friends more opinions, vocabularies and English expressions. (42U-J)

I enjoyed discussing and then giving our opinions so we could combine opinions for some problems. (16-U-J)

I enjoyed the group work. I liked to hear opinions of other members and especially the speaking style of the senior student. (32-A-J) [Translation from Vietnamese]

The senior student helped me with more knowledge and experience about working in the group. (36-A-J)

Few students reported a link between stress and learning. Five students in the unassisted groups and two in the assisted ones reported in their journals that they had felt stressed because they had had to contribute and share ideas with other members (see section 10.5). Stress in this sense was the result of participation in group work.

Interaction in peer groups or in peer groups with the more advanced peer led to reports of learning. The students were pleased to be introduced to new ideas or knowledge of the topics under discussion. They enjoyed learning and sharing ideas with other members. However, the process of sharing ideas and contributing to the group work 
also caused stress for some students speaking English. The assisted students also enjoyed learning from the senior student.

\subsection{Other Feelings}

Emotional reactions are not restricted only to enjoyment and stress. In the interviews, I asked the students, "In what ways was your experience in the group work with the senior student different from without her?" The students reported different feelings rather than enjoyment and stress. Their responses presented in this section were translated into English. Eleven students in the unassisted groups reported their positive feelings. Seven reported feelings of being at ease and of being natural among friends. Three stated that they felt independent and active, and one felt it was easy to speak.

Without a senior student, we did not follow any order. We did not discuss the topic in depth but we were more natural and spoke more. We were cheerful and when we could not express ourselves, we spoke Vietnamese. (24-I)

Other comments were;

The senior student helped me to think but I was passive. (21-IU)

Without a senior student, we did not know how to start a conversation. However, it was easy for me to speak. (26-IU)

Without a senior student, I spoke short sentences and it was more difficult to express my opinions. I was less comfortable. (10-IU)

The discussion was not focused and I was less confident. (15-IU)

Twenty assisted students reported positive feelings of working with a senior student. The senior student helped them feel more confident (nine responses), motivated (five responses) and comfortable/cheerful (five responses). Examples follow: 
Without a senior student, I was not confident. The group work with friends was less exciting. The senior student helped me with vocabulary and mistakes. (04IA)

With a senior student, I was more confident and motivated. I spoke more. (38-IA)

However, not all the students reported that they liked the assisted group work. Working in the assisted groups caused feelings of being dependent on the senior student, passive (three responses), and afraid of making mistakes (two responses). Here are some examples translated from Vietnamese:

The senior student contributed ideas but I did not want to think. I understood more but she gave all the suggestions. (42-IA)

When we were together with friends, we had to do our best. But when there was a senior student, we relied on her. (02-IA)

She led us to the focus of the task and the discussion was interesting and not stressful. However, I was afraid of making mistakes. (35-IA)

The reports somewhat showed the dependence of the students on the senior student.

The students in both the unassisted and assisted groups reported both positive and negative feelings. While some of the unassisted students enjoyed being natural among friends, others felt more confident doing the task with the help of the senior student. The students had been in the same class for their first year at university. The students' enjoyment varied according to which group they were in and the topics to be discussed. More assisted students reported positive feelings. On the audio tapes, the senior student could be saying things, such as: "That's good", "It's a great idea", or "Wow!" 


\subsection{Discussion and Conclusion}

This chapter presented data from the audio transcriptions, students' journals and interviews. It reported the students' feelings during group work in the two different group settings and found differences according to group setting. There were many instances of laughter heard on the audio tapes. The journals were the source of the students' reports on their enjoyment and stress and the reasons why the students had these feelings. However, the interviews when conducted face to face revealed more details about other feelings. In general, the students in the both types of groups reported enjoying group work rather than feeling stressed. The students enjoyed exchanging understanding with group members, discussing certain topics, and having opportunities to speak. In addition, most students enjoyed the assistance of the senior student. However, working in groups also caused stress to some students. In the interviews, some unassisted students reported feelings of being natural and comfortable among friends but also less confident and stressed. Some assisted students experienced being more confident and motivated while others felt dependent on the senior student, or afraid of making mistakes. The students' English competence could have influenced their feelings of enjoyment or stress during group work.

The data presented in this chapter report emotions in the process of learning English, and suggest the relation between learning and emotion, and between culture and emotion. The students reported that they enjoyed learning new ideas from the discussions. They enjoyed the group work because their language skills were improved and because they had opportunities to use English and learn new English words. However, the process of sharing and learning ideas from other members could cause 
stress among some students. The students laughed when discussing familiar Vietnamese cultural practices and beliefs. 


\section{Chapter 11}

\section{ENGLISH OUTSIDE THE CLASSROOM}

\subsection{Introduction}

According to Candlin and Mercer (2002, p. 1), learning in the classroom is tied to learning out of the classroom:

No language teaching and learning takes place however, in a classroom which is isolated from the world of experiences and personal engagements and investments of learners outside the classroom itself. In that sense the wider social context of life outside the classroom has an important effect on what takes place in these interactions between learners and teachers, and among learners. For many learners, the contexts outside the classroom are not only where they make use of the English they have learned in class, but they can also constitute a powerful incentive (or disincentive) for further learning.

This chapter answers the research sub-question, "What support for the learning of

English is provided by the Vietnamese environment?" It reports what the students said about why they were learning English and their opportunities for learning English at home and in the community. All their responses reported in this chapter were translated from Vietnamese.

\subsection{Motives to Learn English}

In the interviews, I asked the students, "Why are you learning English?" Students gave several reasons for choosing English as a major at university. Most of the students said that they were studying English due to extrinsic factors, such as jobs and exams. Twenty-five of the students chose to study English because they wanted to look for a job that required English. In Vietnam, working in the areas that require English 
generally brings higher salaries. One intrinsic factor, reported by twenty-two of the students, was that they liked the language. Another factor that affected the choice of English was that English is compulsory for the high school graduation examination. In Vietnam, students have to pass the high school graduation exam so that they can take the university entrance exam. Eleven students chose English because they were more confident with English than with other subjects. The students also learned English to be able to communicate with foreigners (10 responses), to read English materials (six responses), to travel abroad (six responses), and to learn about other cultures and peoples (six responses). Four students reported that they wanted to learn English to sing English songs, probably popular songs, and one learned English to be able to learn how to use a computer.

The students were driven by both extrinsic and intrinsic factors to learn English. They learnt it for jobs, exams, social communication and also for their own enjoyment of the language.

\subsubsection{Choosing English as a Major}

When interviewed, "Who was influential in guiding you in majoring in English?" Most students named more than one source. Thirty-three students answered that they had themselves decided to take English as major subject at university. Family members encouraged and advised them (17 responses). Students also listened to advice from their teachers (10 responses) and friends (5 responses). Some examples follow,

When I was at high school, my English teacher advised me to choose English as a major at university. (07-I) 
My English teacher is also my aunt. She encouraged me to choose English as a major. And my mother also encouraged me. (10-I)

I chose English as a major myself. My parents encouraged me. My friends also advised me. (24-I)

The answers suggested that the choice of English as a major was commonly discussed with family and friends and that teachers were likely to offer advice.

\subsection{Learning English Outside the Classroom}

\subsubsection{Vocabulary}

In Vietnam, where English is a foreign language, learners do not acquire vocabulary in a natural way as children do with their first language. However, vocabulary is not a subject at university and students are not assigned vocabulary learning as homework. I asked the students, "Do you learn vocabulary at home? If yes, how do you learn?" The answers from the students showed a variety of ways. Thirty-three students reported that they wrote down on paper new words from their readings or from classroom materials, notebook or the board in order to learn them. In the students' responses, writing words down was the dominant way of learning English vocabulary. Dictionaries helped with learning vocabulary. Seventeen students said that they checked with a dictionary to learn new words to expand their English vocabulary. Seventeen students learned English words through reading. Fifteen students practised learning by heart or reviewed words every day. Seven students reported that they talked to friends or teachers out of the classroom in order to learn vocabulary. A few students mentioned making sentences with words or doing vocabulary exercises (seven responses), listening to English music (four responses), or English radio programmes (one response). Two students read the signs and advertisements in English in the streets in the city where 
they lived in order to learn words. Some advertisements for beer, food and tourism were written in English. One student labelled objects with English words in order to learn them.

The students all used writing, reading, speaking and listening skills in order to learn English words. They all made use of books, newspapers, dictionaries, songs or even advertising posters in English.

The data in Chapter 8 revealed the processes of learning vocabulary in the classroom, where interaction and negotiation with group members was shown to be a way to learn words meanings and forms or to help others with words. The students' responses in the interviews as presented in this section revealed the individual ways of learning vocabulary. When outside the classroom, the students had to depend on themselves and what the environment offered them to expand English vocabulary. Writing words on paper or checking words in a dictionary did not require another person to speak to. These artifacts were the dominant support for learning English vocabulary outside the classroom.

\subsubsection{Reading}

I asked the students, "Do you read at home? What materials do you read?" Thirty-nine students read books on different subjects, newspapers, and magazines in Vietnamese. Twenty-eight students said that they read bilingual (English and Vietnamese) materials that included some books, newspapers and magazines published in Vietnam, such as Sunflowers, Special English and Easy English. In English, the students most often read reference books (17 responses). Eight students read an English magazine Heritage 
published by Vietnamese Airlines for passengers to read during a flight. They also read novels and stories in English which were not part of their course (seven responses), test practice books (five responses) such as TOEFL (Test of English as a Foreign Language) and the Cambridge Certificate Tests. These test books were not compulsory at university. Two students said they read French textbooks. French was a compulsory subject for these English major students. However, the majority of the students did not think that they were able to read in French.

The students did not seem to read a variety of materials whether in Vietnamese, English, or both because they had limited access to them and there were not many resources in the city where the research took place. It could be that the availability of or access to reading materials was limited, or the students did not want to read a lot. The students' responses on the materials that were bilingual or in English showed that there were few kinds of materials available to them.

I also asked the students, "Do you find the materials yourself, or were they recommended to you?" "Materials" had a broad meaning. They included whatever the students read. Overall, 38 out of 45 students reported that they looked for materials themselves. Twenty-two of them had friends who introduced them to reading materials or who lent them materials. Sixteen students had materials recommended by teachers and eight by family members. 


\subsubsection{Resources}

When asked, "What learning resources do you have at home?" the students listed different resources as presented in the following table:

Table 5: English Learning Resources Outside the Classroom

\begin{tabular}{ll}
\hline Sources & $\begin{array}{l}\text { Number of students' } \\
\text { responses }\end{array}$ \\
\hline Books & 45 \\
Cassette players & 42 \\
Audio tapes & 27 \\
Materials/Newspapers & 13 \\
Computers & 12 \\
TVs & 11 \\
Music tapes/programmes on radio & 9 \\
Video movies with subtitles & 6 \\
Dictionaries & 5 \\
CDs & 2 \\
\hline Note: A student could report more than one kind of learning resource. \\
N of students = 45.
\end{tabular}

All the students interviewed had books to study both in English or English and Vietnamese. Forty-two of them listened to tapes and the radio. For radio programmes, seven students reported that they listened to the VOA (Voice of America) special English programme, three to the BBC (British Broadcasting Corporation) programme, and six to the FM English programme broadcast from Vietnam. Forty-two students also used cassette players to listen to English tapes at home. Thirteen students reported that they read newspapers and printed materials other than books.

A few students had access to English through TV, computer, videos, and movies. Overall, the students relied mainly on printed materials and they rated books as the most important source for learning English at home (20 responses), cassettes as the second (13 responses), and audio-tapes as the third (11 responses). 


\subsection{Using English outside the Classroom}

\subsubsection{Using English with Foreigners}

Most of the students in the interviews reported that they had opportunities to use English with foreigners outside the classroom. Only four of them did not. Twenty-six students went to speak to tourists at historical sites and public places, such as railway stations and hotels. Some students reported that their houses were near tombs and pagodas and that they had opportunities to speak to foreigners there.

My hometown is a tourist spot in Hoi An so I have opportunities to speak with tourists. (29-I)

My house is near Linh Mu pagoda which a lot of foreigners visit and so I often talk with them. (37-I)

At the tertiary institution where this research was carried out, foreign volunteer teachers had come to teach the students English. The students thought that talking to them was a good way to practise the language. Fifteen of them reported going to the foreign teachers' houses to talk to them.

When the data collection for the current research was being carried out, a cultural festival of nine Asian countries and France took place in the city. The festival is held every two years. Eight students reported that they volunteered to help the tourists coming from all over the world by providing information about the festival. This gave them a chance to use English with foreigners. The students had little access to computers or the Internet at home and only one student reported that he/she used the Internet for learning English. However, seven students reported that they went to Internet shops where they could chat in English to foreigners. In Vietnam, Internet 
shops are popular and chatting on the Internet is a popular means of entertainment among young people.

Three students reported that they had had a chance to translate a letter into English and to speak on the phone in English with relatives abroad. However, one student honestly and bravely reported that speaking to foreigners was not always successful.

I sometimes talk to tourists but usually they do not give me the opportunity to speak. They usually walk away. (38-I)

\subsubsection{Using English with Vietnamese Friends}

While 34 students reported that they used English with friends who were not necessarily their classmates, eleven of them reported that they had never used English with friends outside the classroom. Twenty-four students used English to talk to Vietnamese friends in daily conversation, when they went out together, or when they greeted friends from other classes, classmates or roommates in English. Nine students wrote letters in English to friends. Four of them participated in a conversation group and met weekly to talk. The topics were those that they studied in class. Three students used English to communicate with Vietnamese friends through the Internet.

I also asked the students if they thought speaking English outside the classroom was important. Forty-four students interviewed considered that speaking English outside the classroom was very important. One said "no" because he/she did not think there were speakers to talk to. The students gave different responses for their "yes" answer. For example, most students considered speaking outside the classroom helped them improve their speaking skills (23 responses). They also thought that it could provide 
situations and topics that triggered English learning and use (18 responses). Speaking English outside the classroom was considered to bring the students more confidence and independence (17 responses). Five students wanted to prepare themselves for future jobs through participating in practical situations. As the students were learning English as a foreign language, three of them reported that when talking with foreigners, they could learn about them and their cultures. One student wanted to be an interpreter.

\subsubsection{Speaking English at Home}

When asked if they spoke English at home with family members, 15 students said "yes" and 30 said "no". The two thirds who replied negatively had family members who did not speak English at home. Those who had family members speaking English at home had a chance to speak English to their family members. However, they did not speak English very often, and generally used only some common and simple expressions. All of them reported that they sometimes, or once in a while, used some common English expressions at home.

\subsection{Summary}

This chapter documented the findings on the learning of English outside the classroom and showed that the students did have some access to both written and oral English in the wider Vietnamese society but that their chances of speaking English to native speakers were limited. The interview reports provided a background of why the students learned English and the resources available for them for learning and using English at home and in the community. Factors, such as employment, and the 
requirement of English in the high school graduation exam, to a large extent, determined the choice of learning English among the students. Some students chose to major in English because of their interest in the language or for communication with foreigners. Parents, teachers, relatives or friends could influence their choice of English as a major at university.

Outside the classroom, the students learned English vocabulary, read materials and sought opportunities to speak English with foreigners or friends. The students had access to a few resources in the form of physical artifacts, such as reading materials and audio visual aids for learning English. The majority of the students had books as a primary resource for learning and considered books as the most important and useful learning aid. However, they showed high motivation to seek every chance to use English whether with Vietnamese friends, or with English speaking foreigners. 


\section{Chapter 12}

\section{DISCUSSION OF OVERALL FINDINGS: THEORETICAL CONTRIBUTIONS AND PRACTICAL IMPLICATIONS}

\subsection{Introduction}

My study used sociocultural theory as a conceptual framework to analyse and interpret the English learning processes in group work. Vygotsky argued that psychological and material artifacts mediate the construction of mind. As part of this process, he proposed the concept of the ZPD but did not explain how a learner moved through the ZPD beyond referring to the agency of more knowledgeable others who were either their peers or were as teachers. Sociocultural theory as it has been applied to classroom studies focuses particularly on peer interaction in small groups and dyads. This raises the question of whether there are differences either in the roles of peers, and in social interaction or in mediation, according to the characteristics of the members of a group, and to the context (Le, 2003b).

This study was carried out in a university classroom in Vietnam. Participants were 45 first year students majoring in English. The class normally spent 20 minutes practising English in groups of five students, using a textbook which set the topics for discussion. Because I wanted to study the sociocultural processes in two different kinds of groups, I reduced the number of students in 12 group sessions to four students from the same class and introduced a senior student from an advanced class to work with them. This resulted in groups which were called "unassisted" because members were all from the same class, and groups which were called "assisted" because they included a student from a more senior class. All the students experienced working in both an unassisted 
and assisted group. The two kinds of groups differed in composition but had the same task, which was to make statements in English relevant to an assigned topic. In the following sections, significant findings and key themes emerging from the current study are discussed. First, the thesis is summarised by presenting the similarities and differences of the processes which were observed in both kinds of groups.

\subsection{Similarities}

Whether in the unassisted or assisted groups, the students had opportunities to speak and to negotiate the meaning of the themes set for the discussion in the target language they were practising. The audio transcriptions of the data showed that while working in groups, the interaction gave students opportunities to: 1) express themselves in English; 2) help each other with task planning and procedures; and 3) to share their understanding of the topics under discussion. The students came to the discussions with their own subjective ways of making sense of it. They discussed differing viewpoints and made their perspectives known (see sequences 02-16, Chapter 7 and sequences 20 , 24 and 25 Chapter 8 ). In the process of their discussions, all students showed support for others. The group members attended to one another's points of view and used them to stimulate their own ideas. They stretched their common understanding to fit in with new perspectives in the shared endeavour as set out in the tasks that they were required to complete. Records of students' discourse indicated their understanding of the topics under discussion and the English they had learned. The process of negotiation of understanding in the group work had features of "collective scaffolding" (Donato, 1994) and "collective co-construction" (Jordan, 2003) in which members drew on the resources from group members. 
Working in unassisted or assisted peer groups, the students reported that sharing ideas among group members was a factor that created a successful or satisfactory discussion (section 5.3, Chapter 5). When they felt that the discussion was ineffective, it was generally explained as due to time constraints, being unclear of the required tasks, and limited English proficiency. In the process of negotiation of understanding, the students shared their understanding by building up, elaborating, adopting, and expanding ideas from other peers. The meaning, which came from the knowledge of each individual, was accumulated from previous learning, classroom artifacts, and what had been learned outside the classroom. The meaning also came from the written language provided in the text and the instructions on the blackboard. Although the principal aim was to practise English rather than acquiring knowledge, the students in both types of groups reported learning of new facts (section 5.3.3 Chapter 5).

As reported in their journals (section 8.5 , Chapter 8), during participation in group work, peers helped each other to learn English words that they had not known before. The students reported that they found it beneficial to study in groups because it provided them with an opportunity for peers to help them understand the meanings of the words that they did not know, and to correct them when words were used incorrectly (sequences 20-26, Chapter 8). Participation helped activate the receptive words which they had learned but might have forgotten, or did not know how to use (section 8.5.3, Chapter 8). As group members explained a particular point, they played a mediational role in helping others understand words. They understood what other peers needed and gave them opportunities to use the words by engaging them in the negotiation of words, meanings and forms. For example, a student might correct the 
pronunciation of another student or translate an English word into Vietnamese to explain the meaning.

One of the issues in sociocultural theory applied to classrooms is that while we can often tell what those who speak know, it is not so clear what non-speakers have learnt (Larsen, 1999). While some of the less assertive students did not speak very often, there was evidence that some students were learning as "overhearers" (sections 8.5.1 and 8.5.3, Chapter 8).

Outside the classroom, all students reported limited access to materials and opportunities to use English. However, they were highly motivated to improve their English through, for instance, learning English vocabulary, reading English printed materials, and practising speaking English. Most of the students were studying English with the aim of future employment and also for the love of the language. They sought opportunities to use English with English speaking foreigners as well as with Vietnamese people.

\subsection{Differences}

Because students had a fixed amount of time to practise English and the senior student took up some of that time, the students in the assisted groups did not have greater opportunities to speak than they did in the unassisted groups. However, the assisted groups completed more tasks and were more efficient in discussing a textbook topic than the unassisted groups. During the group work, the unassisted students produced more Vietnamese and combined language utterances. They used Vietnamese when they 
had to figure out what they had to do, or when they needed explanations for the English words that they did not know. They also produced utterances which were off the assigned topics. The process of sharing understanding in the unassisted groups was not always successful. The students sometimes went off task and made fun of each other (sections 7.3, Chapter 7 and 10.5, Chapter 10). There was also evidence that on occasion they ignored each other's contributions (sequences 17 and 19, Chapter 7). For example, if a question was raised by a group member, it was often left unanswered. Failure to respond may have been because of insufficient knowledge of the topics under discussion, lack of English vocabulary, or lack of confidence to speak.

The patterns of utterances in unassisted groups were irregular whereas the patterns of the assisted groups were mainly dyadic and consisted of exchanges between the senior student and individual members of the group in turns. The unassisted students spent more time working out how to do a task. In some sessions, the unassisted groups experienced confusion about what they were supposed to do (section 7.2.1, Chapter 7). In the assisted groups, the senior student helped the students to understand the required tasks and to start the discussions. The students in the unassisted groups received help from other group members. The assisted students received help both from the senior student and from the other students.

Van Lier (1996) considered that peers working in groups demonstrated "communicative symmetry", showing equal respect for the voices of the participants because the group members are equal in status and proficiency. The transcriptions of the current study showed that the discussions were not always of this kind and that there were examples of domination by a particular student in the unassisted groups. 
Whoever was most assertive would speak more often. Some students in the unassisted groups tried to perform a teacher role but this was not always successful.

In the assisted groups, the senior student engaged the students with IRF (InitiationResponse-Feedback) exchanges (van Lier, 1998, p. 163). She asked a question. The members in the assisted groups answered individually and the senior student gave them some feedback. She was also a source of information and clarification. She encouraged the students to reason, a factor which was absent in the unassisted groups. In this way, the IRF exchanges in the assisted groups sometimes moved beyond mere recitation and display. The senior students' questions can be regarded as mediating questions. IRF has a variety of pedagogical implications, although it has tended to be dismissed as less valuable than, for example, co-operative group learning (Van Lier, 1998). The present study indicated its value for the students in the small groups.

The comparison of the processes in both types of groups revealed that the students in both group settings were able to complete the assigned tasks. Overall, the assisted groups were able to use more English and their discussions were more task-focused and efficient. The unassisted students lacked task structure and often experienced confusion.

The following section discusses the first theoretical issue which emerged from the current study. 


\subsection{The Roles of Peers in Different Group Settings}

In the current study, peers worked differently in the different group settings. The students generally reported that they benefited from the group work, whether they were unassisted or assisted. Peers created a comfortable and friendly atmosphere in which to share ideas (sections 5.3.1 and 5.3.2, Chapter 5). Peers were ready to help, and were sensitive to the needs of group members and in learning vocabulary (Chapter 8). However, peers could also ignore and put group members off-task (sections 6.3, Chapter 6 and 7.3, Chapter 7). Peers worked as discussants in group work and knew how to engage other peers in the discussions. Peers were addressed as friends. In the assisted group work, peers accepted that the senior student was more knowledgeable than they were. They did not challenge her as they sometimes challenged fellow students in the unassisted groups. At the same time, they attended to the contributions of other peers. In either kind of the groups, peers adopted different responsibilities. Some more assertive peers worked as group leaders. The less assertive peers might then become the audience.

The findings showed that the more knowledgeable peer behaved much like a teacher (Chapter 7). She directed and controlled the process of group work. She established the order of the turns and managed the timing by inviting group members to speak. In working with the students, the senior student

1. encouraged students to offer opinions on the topics,

2. assisted the students when they needed help with English words,

3. managed and controlled the group work, and

4. focused the students on the task. 
Unlike the help of peers in the unassisted groups, which tended to focus on issues as they emerged, the help of the more senior peer was more systematic and broader. She shaped the discussion by showing the students the procedures for carrying out a discussion. She gave special attention to the less assertive and less competent students. She sometimes spent a longer time in helping a particular student. She tried to engage all students in the discussion. The senior student in the assisted groups drew upon the group members' background knowledge to encourage a variety of responses. Many of the senior student's utterances were questions in a familiar teaching style. Although the assisted students had relatively fewer opportunities to speak, they had the opportunity to observe how to form questions in English.

In the group discussions in the two settings, there were more knowledgeable peers who scaffolded other peers on issues as they emerged, for example, task planning, explanations for new words, and ideas for responses to the questions in the textbook. The scaffolding process reflected what sociocultural theorists have pointed out (see section 3.4 , Chapter 3 ) that the more knowledgeable peers were able to guide and help other peers to do what they were not able to do by themselves. However, peers did not assist other peers to solve a problem in an organised and oriented manner as described in many accounts of scaffolded talk between teachers and learners and between mothers and children. Peers were sensitive to other peers but did not recruit the interest of other peers in carrying out the tasks. Peers did not praise other peers. In addition, their help depended on their available knowledge which might be correct, or, in some circumstances, might be incorrect. For example, in the current study, unassisted students sometimes provided incorrect information about the required tasks and offered some incorrect meanings for English words. Their scaffolding strategies depended on 
what they knew. When they failed to use English to figure out what they had to do, or to help their peers, they relied on the mother tongue. In other words, the mother tongue was often used as a scaffolding strategy among the unassisted students but very rarely in the assisted groups.

In comparison, the scaffolding strategies used by the senior student were more efficient. She knew how to highlight important features of the required tasks. She demonstrated her knowledge by asking questions and giving responses to questions in English. She demonstrated how English could be used to manage the task as well as to hold a conversation. She played a key role in starting a discussion in the assisted groups, a task which appeared to be difficult for some unassisted groups. When a student was searching for a word, she scaffolded the student by providing examples and checking understanding. As a result the student was able to use the word to complete her/his response. She organised the group work in keeping with her view of herself as a teacher. She praised the students when they gave responses. The senior student's knowledge of the topics and her teaching manner made her scaffolding strategies more successful. The students accepted her authority, and not solely her knowledge, in responding to her scaffolding.

The ZPD was demonstrated as both a process and an outcome in the current study. As a process, peers assisted each other with the tasks that they could not do individually. They cooperated to work with the tasks which required English comprehension. In the discussion planning phase, peers clearly demonstrated their collaboration in figuring out what to do. During the discussions, the more knowledgeable peers used scaffolding strategies to assist other peers. They identified the difficulties experienced by other 
peers and offered help by offering ideas and suggesting English words. Their knowledge of topics discussed and their English speaking skills were activated. The outcomes of the ZPD were shown in the knowledge of vocabulary documented in the audio transcriptions and students' journals where the students reported that they learned certain words they did not know before taking part in the group discussions (see Chapter 8). They also reported that their knowledge of the topics under discussion was expanded (see Chapter 5).

Sociocultural studies have supported the active role of learners and, particularly within the constructivism movement, there has been distrust of a teacher-led, so-called transmission teaching method which views the teacher as the source of knowledge to pass on to the students. To some extent, the senior student's style of operation was similar to transmission teaching. She possessed more knowledge and she passed it on to members of her groups. She controlled the group processes. However, she made a marked difference to the organisation of the assisted group processes as well as providing direct help in the form of getting the students started on their discussions, keeping them on task and explaining new English words. She helped them to use English more often. When working in the groups without her, the students sometimes experienced confusion. They took more time to understand exactly what they had to do. They also discussed matters which were off the topics. The independent work of the unassisted groups tended to lack group work skills. It could be argued that a teaching style contributed substantially to the group work in the current study.

The findings of the current research on the teaching and learning styles between a more capable peer and other peers were similar to the findings from McDonald et al. (1991) 
and Barnard (2002) although these studies were carried out in different periods of time and with students from different cultures. Does this indicate the universality of learning-teaching interaction? Or that "the search for both universal and culturespecific forms of mediation is only beginning" (Kozulin, 2003, p. 29)?

\subsection{The First Language Use}

In sociocultural studies, oral language is generally presented as a mediating tool for thought (Mercer, 1995; Wells, 1999). In learning a foreign language, both the mother tongue and the target language have mediational roles in the interaction and learning in the classroom because second/foreign learners cannot switch off the first language when learning another (Cook, 2001).

In the current study, the students, especially when in the unassisted groups, used Vietnamese in order to figure out what they had to do. On occasion, Vietnamese helped them to express their ideas in English. Vietnamese facilitated the discussions in English. Several reasons for the use of Vietnamese in the unassisted group work were apparent. First, it can be assumed that the students in this research were more competent in Vietnamese than in English. Second, the unassisted students had experience of carrying out many similar tasks using the Vietnamese language, for example, in working on task planning. Their linguistic competence in Vietnamese was fast and reliable. Third, the process of working with task planning and procedures was challenging, and often beyond the current level of competence of the students. The students thus used Vietnamese to overcome the task challenge: speaking English on specific topics and performing according to the rules of group behaviour. Fourth, the 
students used Vietnamese when their English vocabulary seemed to fail. In this way, the use of Vietnamese facilitated the thinking processes but denied the learners opportunities to use English which was the purpose of the groups.

Kobayashi (2003) pointed out that learners of the same mother tongue tended to use their first language when working together. The current study showed that when the students were working with the senior student, they hardly used Vietnamese. On the contrary, the students of the same level in the unassisted groups used Vietnamese more often. This suggests that an equal relationship among peers using the same mother tongue is also one of the reasons why learners used the first language. It could be that when working with peers of the same level, the students had to spend more time figuring out what they had to do. Therefore, Vietnamese was needed when the students were under the pressure of time.

The particular topic played a role in whether the first language or English was elicited. The unassisted students used the first language when the topic was relevant to their own experience as, for example, when it was about cultural beliefs.

Anton and DiCamilla (1998) advocated the use of the first language in the classroom as a psychological tool to meet the demands of the task of learning a second language. Lantolf and Appel (1994) similarly claimed that the first language can support second language. The current study findings raised the issue of how much the first language should be allowed in an EFL classroom. In the context of learning a foreign language in Vietnam, where the main use of English is in the classroom environment, it is necessary to consider the proportion of the first language used in the classroom. If the 
goal of a language activity is to speak English, then a figure of some authority, similar to a teacher, encourages the use of the target language.

Vygotsky (1978) considered language as a psychological tool or a tool of thought but this definition applies to learners learning their mother tongue. In learning another language, more explanation is needed. The data from the current research showed that with EFL learners, language as a psychological tool is associated with familiarity with the tasks. The students were using English to express ideas already in existence and understood within their first language. English tended to be used as an instrumental tool to convey and express the Vietnamese meanings that the students wanted to share with other peers.

\subsection{Social Interaction for Task Management}

As already mentioned, sociocultural studies have focused on the role of talk and how knowledge is constructed through talk (see section 3.3.1, Chapter 3). The goals of talk have generally been interpreted in terms of cognition. This emphasis on the content of talk tends to overlook the management process of discourse. In the current study, talk was not only a means for social interaction and sharing understanding among peers but also a tool for planning. It was used to figure out what a task entailed and what steps needed to be taken to complete the task successfully, using procedures already known but applying them to new situations. Time was spent in organisation and getting started in both kinds of groups, and this took longer in the unassisted peer groups. At least some of the social interaction in groups involved seeking agreement on management rather than knowledge. The result was action rather than knowledge. 


\subsection{The Roles of Artifacts in the Classroom}

Little research gives insight into the way that more traditional resources such as textbooks are used during particular collaborative learning tasks and into the way that such use affects the content and patterns of student talk and the learning outcomes. (Van Boxtel et al., 2000, pp. 59-60)

Mediation through artifacts has certainly been discussed in sociocultural studies. However, research within sociocultural theory tends to focus more on the mediational role of oral language. The current study shows that speech is insufficient to explain the processes of social interaction in group work. In this study, the artifacts existed in different forms: the textbook, classroom handouts, instructions on the blackboard, sheets of paper, notebooks and a dictionary. All contributed to the group discussions and their outcomes.

The textbook played an important role in shaping the students' task structures and thoughts. The two main mediating roles played by the textbook, as shown from the audio transcriptions (section 9.2.1, Chapter 9) included: providing mediating tools for thoughts or ideas and acting as a guide to the language tasks and activities. In playing the role of a mediating tool, the textbook made the students think about the situations described and led to interaction and sharing ideas from the students. In playing the role of a guide, the textbook determined the structures and the content of the discussions. It gave the students directions to follow. Whether in the unassisted peer groups or in the peer groups with the senior student, the students used the textbook as the main source of information. The students read out the situations provided in the textbook. They then responded according to their understanding of the topics. The students referred to the textbook for instructions about the task. In other words, the textbook in the form of a 
material artifact worked as a conceptual artifact to mediate their thinking and forming of ideas. The textbook expressed "intentions", for example, creating contexts and situations for the language activities and constraining the participants' actions.

In the different group contexts of being unassisted or assisted, the textbook was used slightly differently. The textbook guided the unassisted students, whereas in the assisted groups, the textbook was used with the interpretation of the senior student. The students in the assisted groups read the textbook at the same time as the senior student was reading from it and explaining what to do. The video tapes also showed that all students relied on the textbook as a guide to give them direction on what to do, for example, they read out the topics to be discussed, located the questions in the textbook and searched for relevant information.

Van Boxtel et al. (2000) in their study found that when students had to use a textbook for information, it was an impediment to group learning, because its use "constrained elaborative interaction and co-construction". However, the textbook in van Boxtel et al.'s research was used as a source of reference information. It was not an integral part of the lessons. The current study finding, to some extent, is similar to the findings of Hill et al. (2003) in that the artifact structured the participation and the topic to be discussed.

In comparison with the textbook, other artifacts, such as a travel brochure, a pen, and an imagined exercise machine did not seem to determine the structure of the discourse of each respective discussion. Nevertheless, the students used them as additional resources for discussion. In the session review, the students also relied on the textbook 
but as storage of relevant information. The nature of the task also influenced the way an artifact might be used.

The instructions written on the blackboard were examples of written language. The data from the videos showed that reading and understanding the instructions on the blackboard were more important for the unassisted students. During group work, the unassisted groups read the instructions on the blackboard more often. In addition, they wrote on paper and in notebooks more often than did the assisted students.

This research illustrates the roles of speech. It also recognises the mediational roles of classroom artifacts, and especially the textbook which contains written language. It argues that the textbook played an important role in shaping students' thinking in response to the questions it posed and influenced their interactions in group work. As an artifact, the textbook offered the students both "affordances" and "constraints" (Wertsch, 1998, p. 38). It gave the students topics to be discussed and scenarios which required thought. It also constrained their thinking when it asked the students about experiences beyond their knowledge. For example, the students found it difficult to talk about laws in Britain or how to choose and describe a vacation.

In summary, the current study has provided information on the role of the textbook in engaging students in classroom learning and in mediating their thinking. In addition to the textbook, the students used other artifacts and referred to the instructions written on the blackboard for information and guidance. In this way, mediation occurred when students were using written language for oral interaction. 


\subsection{Mediation as a Complex Process}

Mediation is a central concept of sociocultural theory. When the fieldwork was planned, a research model was developed with a unit analysis of mediated group work (see Figure 1). As the study progressed, mediation by groups emerged as a complex process consisting of different layers, which were interconnected. Mediation occurred through both oral and written language and through the language tasks assigned. The students and the classroom teachers were driven by the goals as set out in the curriculum and in the textbook. The instructions of the teacher mediated the processes of group work. The senior students' questions further mediated the students' thought related to the textbook questions. The textbook mediated the adaptation and assignment of tasks by the teacher to students. It also mediated the students' responses to the questions that it posed. Peers mediated each other through their statements and questions. The first language mediated the students' ideas which were presented in English.

In the form of oral language, mediation occurred in the processes of negotiation. Through negotiation, the students arrived at solutions and responses to others and to the questions in the textbook. The mediation process in the assisted groups occurred mainly between the senior student and other peers and partially among peers. The senior student played the key role in shaping the discussion structures. The students in both types of groups at times exchanged input and output of language. However most of the time they were using oral language as a means of regulating themselves, in the flow of the discussion, and to ignite thoughts and responses from other peers. 
In the form of written language, the instruction on the blackboard and the questions in the textbook guided the students and generated the students' thoughts. The assigned tasks mediated the ways in which peers talked and used other artifacts.

The different layers of mediation can be summarised in the following flow chart:

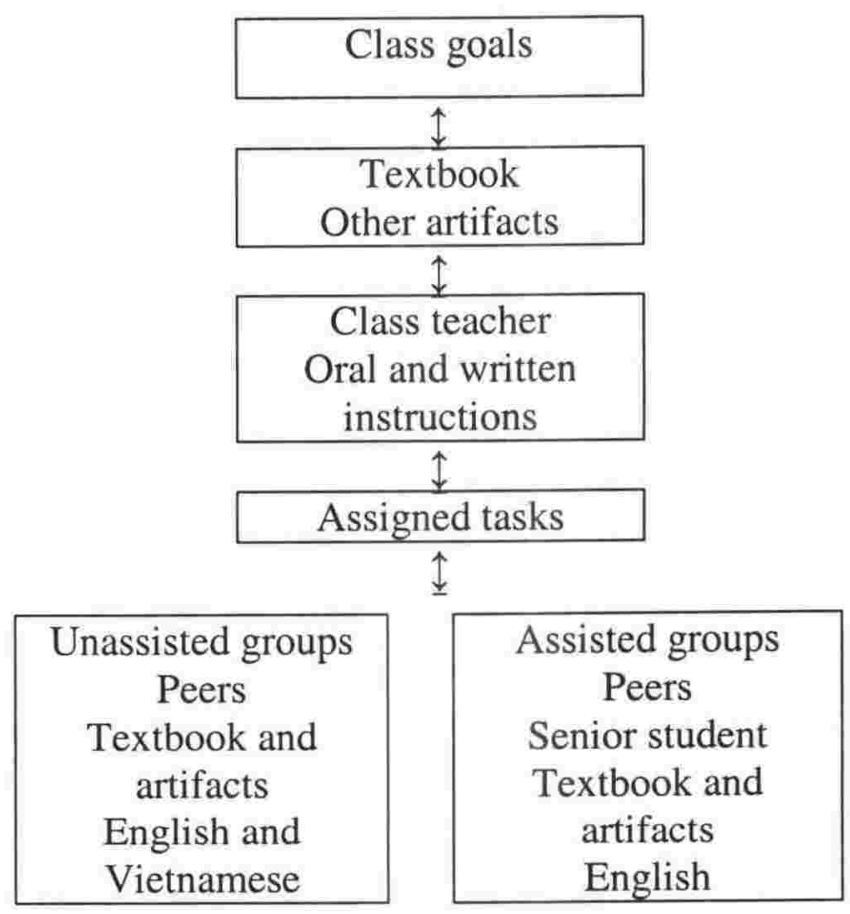

Figure 9: Mediation in Group Work

\subsection{Emotions in Participating in Group Work}

It appears that sociocultural studies have tended to underestimate the importance of affect, and so the current study contributes insights about students' feelings in two group learning conditions. The data collected on affective factors revealed that there were emotional factors when working in groups (Chapter 10). The students experienced different kinds of feelings. For example, they reported both enjoyment and stress when interacting with group members or with the senior student. The feelings resulting from participation in the group work varied according to the type of group the students were 
in, the topics to be discussed, as well as required tasks. The students said that they enjoyed learning new ideas, sharing ideas, and improving language skills.

Overall, the students experienced more enjoyment than stress during group work because they liked being helped by other group members and having an opportunity to express their own ideas. Some students in the both types of groups reported that they felt stressed because they had to concentrate and contribute ideas to the group discussion (section 10.5, Chapter 10).

The pattern of laughter was similar in the two kinds of groups. The students laughed at the ideas raised by the tasks, for example, exercising and getting married. They laughed at familiar practices of Vietnamese culture, such as eating, and beliefs of good luck and back luck (section 10.2, Chapter 10). Working in either unassisted peer groups or peer groups with the senior student was likely to produce laughter. The sequences shown in Chapter 10 demonstrated that peers in a group, or the activity itself (in this particular study, a language task) could arouse laughter. The students referred to their own culture and laughed at ideas they were familiar with but probably did not believe. The topics for discussion could arouse the feelings among the students.

The audio transcriptions showed that being among "friends" was a factor that led to more laughter in the unassisted groups (section 10.2, Chapter 10). The assisted students laughed less during group work than the unassisted students. The assisted students laughed when group members were making fun of each other, and when the senior student made humorous comments. 
In the interviews, some unassisted students reported natural and comfortable feelings among friends but also a lack of confidence. Some reported that they gained more confidence and motivation when working with the senior student but others reported feelings of dependence on her (section 10.7, Chapter 10).

As mentioned in section 3.11, Chapter 3, the literature of classroom research within sociocultural theory has not documented the relation between learning and emotions. The students in this research reported in their journals what they learned and how they felt towards their learning. For example, they enjoyed group work because they thought that they learned ideas from other members, shared ideas with others and improved their language skills (Chapters 5, 6, 7 and 8).

\subsection{Vietnamese Culture in the English Classroom}

Like fish in water, we fail to "see" culture because it is the medium within which we exist. Encounters with other cultures make it easier to grasp our own as an object of thought. (Cole, 1996, p. 8)

"Vygotskij's [sic] primary focus in his sociohistorical theory of development was on how human society reproduces itself" (Matusov, 2001, p. 16432). Sociocultural theory examines how cultural practices relate to the ways people think, remember, reason, and solve problems (Rogoff, 2003). However, as in many fields of research, there are several definitions of culture, and in sociocultural theory the definition relates to practices belonging to one's original culture. This emphasis on the influence of culture on human behaviour leaves open the questions of how learners of a foreign language reproduce culture, for example, "Do learners reproduce the foreign language and culture they are learning?" or "Do they use their first language and culture?" In this 
research, the students in both types of groups used their prior knowledge and understanding accumulated from their first culture and language to solve the problems or to give responses to the questions from the textbook written by the authors from a different culture. Whether in the unassisted or assisted groups, the students needed to rely on their own cultural knowledge as judgement for possible solutions to the situations they were encountering. To some extent, their ideas were constrained by what they had learned and obtained from their own culture. As with the textbook, Vietnamese cultural tools and representations offered both "affordances" and "constraints" to the students' thoughts. For example, the students in the current study accepted physical punishment for children's wrong behaviours and regarded teaching as the most prestigious career. They categorised animals as to be used as pets, or as working animals, based on the cultural practices of Vietnam. They talked about the beliefs of good luck and bad luck which were very specific to Vietnamese culture. However, what they had learned from their own culture gave them thoughts which could be expressed in speech. The students had not travelled to any country beside their own. As a result, the first culture and language were the more important sources of information about the topics set for discussion.

The process of referring to the first culture in learning another language can be expressed in the following flow:

External artifacts/representations of the first culture $\rightarrow$ internalisation of first culture through social and cultural forces, formal learning at school, and informal learning in the community $\rightarrow$ culture in mind $\rightarrow$ reproduction of the first culture when learning another language. 
The reproduction process does not mean that the students just produced the utterances without thinking and replayed what was recorded in their minds. In reproducing the knowledge, the students in this research reconstructed what they knew to give answers in English to the questions in the textbook. Their ideas had to be matched to both the textbook and the content of the discussion as it proceeded.

The negotiation of culture as presented in section 9.5, Chapter 9 suggested a difference between the culture of the authors of the book and the culture of the learners. The textbook is an artifact of the culture where it was produced. Learners however applied their own experiences and knowledge to learn with the textbook. The negotiation also indicated the reconstruction of understanding in learning another language based on a textbook from a different culture. The meanings of group discussion were part of the broader systems of relations in which the students lived and studied. Vietnamese culture was vividly reflected in the students' discourse and in the responses recorded in the journals and interviews.

The classroom is a reflection of a broader culture of which values and beliefs towards the learning and teaching process have been built as cultural representations. The senior student in this research was not trained to be a teacher but she talked to the students in the assisted groups like a teacher. She posed the questions and gave suggestions for the students to respond. She started each discussion and ended it. She gave explanations when needed. She and other students had been familiar with the classroom environment in which the "more knowledgeable other" was a teacher. The students in this research would have expected a more advanced student to have certain authority and sufficient 
knowledge to help. In addition, the senior student probably adopted her role as a teacher automatically.

In this research, the notion of a "peer" was perceived as "friend" as shown in the students' responses in the journal entries and interviews (sections 10.3 and 10.7, Chapter 10). The notion of friendship in Vietnamese culture denotes a long lasting and close relationship. Some students in this research could have known each other since high school. They were in the same class in the first year at university. Although "friend" has a more personal meaning than "peer", the students referred to peers as "friends" who worked in the same group. The notion of "friend" that the students in this research used may have a different meaning from its meaning in English.

The finding of the use of gestures in the current research, though exploratory (see section 5.6.3, Chapter 5), contributes to the literature of nonverbal communication in the studies within a sociocultural framework. The gestures produced by the students in this study were commonly practised in Vietnamese culture. The gestures captured on the video tapes include: moving hands, pointing at objects, nodding, pointing at oneself, shaking head and shrugging shoulders. The students used the gestures to attract the attention of other members and to share artifacts and information (section 5.6.3, Chapter 5). The unassisted students produced more gestures, such as moving hands and pointing at objects than the assisted groups. They pointed more often at the textbook and other artifacts, such as the blackboard, pictures, and a pen (section 9.3, Chapter 9). The videos showed that in the assisted groups, the senior student usually pointed to the text for the students to locate the information. The students in the assisted groups 
nodded more often to share agreement with the senior student (section 5.5.3, Chapter $5)$.

The students in this research were learning English in the formal classroom context and they were not taught the gestures used by native speakers. In addition, they were not exposed to gestures used by native speakers in the natural environment. Although the classroom teacher was a native speaker, she worked with the students for only about two to three hours per week. It was difficult for them to move away from what had been internalised in their understanding and communication processes in the first language and culture. The findings supported those by Kellerman and van Hoof (in press as quoted in Negueruela et al., 2003) and Negueruela et al. (2003) that second language learners showed a strong tendency to transfer their first language gesturespeech patterns to their second language performance or to rely on the first language gestures in learning another language.

As seen from the laughter in the audio transcriptions (10.2, Chapter 10), the students laughed because of ideas that were related to the tasks and those that students were familiar with in their culture. The ideas included Vietnamese cultural beliefs and practices, for example, eating, getting married, superstitions, beliefs of good luck and good luck. The data suggested that Vietnamese culture not only mediated the thinking process in learning another language but also aroused feelings in that learning process. In other words, there is a link between culture and emotions. 


\subsection{Group Work as Viewed in EFL Pedagogy}

The values of unassisted and assisted group work as viewed in EFL pedagogical approaches rest on the opportunities to use English and the processes of group work. The unassisted students had to use all the resources given to them in the group work, for example, group members' ideas and classroom artifacts. They produced less English but the members were responsible for their own activities in group work. The assisted students produced more English and achieved more of the goals as set out in the textbook and in the official curriculum. The assisted groups were more task-focused but the students were under the guidance of the senior student. As presented in Chapter 6 , although the students in the both types of groups produced nearly the same average utterances, the language in which their utterances were produced varied. The assisted students on average produced slightly fewer utterances; however, more were in English and on-the-assigned-topic. In addition, they produced more yes-no answers, which were short but facilitated the flow of the discussions.

The success of the discussions was perceived differently by the students from the two kinds of groups (Chapter 5). More assisted students considered the group discussions were efficient because they were structured and focused thanks to the assistance of the senior student. The unassisted students reported more unsatisfactory discussions because of lack of time, structure and understanding of the required tasks.

During the group work, the students learned words through negotiation with group members or with the senior student (Chapter 8). In their journals the students reported learning new words, with more being recorded by assisted students. This does not mean 
that groups of peers of the same level were inferior. The peer groups had a collaborative learning environment in which they collectively helped each other with words. In the journals (Chapter 8), the students in both the unassisted groups and assisted groups reported the specific words that they learned and how they learned vocabulary conventionally. However, the difference was very small. In addition, the journals were based on students' recall and reflection, and not on a verbatim record. Some students may not have reported their experience of learning vocabulary in group discussions.

According to Joe, Nation and Newton (1996), words that were negotiated or used had a greater chance of being learned. Other members in the group who did not report new words could have learned from hearing new words used or explained. "It was not necessary to be the negotiator in order to learn. Learners learned equally well by observing negotiation" (Joe, et al., 1996, p. 4). However, it cannot be confirmed whether the students will be able to use the words learnt during the group work sessions in this research in future language activities or whether they will be able to remember the words negotiated or explained.

Most of the students appreciated their group work experience (Chapter 5). It was an opportunity to learn from their friends' knowledge and language skills (section 5.3, Chapter 5). Although the senior student was a stranger to the students, they had a high appreciation of her roles of a guide to keep them on task and to use the target language. This suggested the role of formal learning in the classroom context. 
Generally, the group work in the current study allowed the students to practise speaking English. It also facilitated interaction among peers and made use of all resources provided. Furthermore, it made learning enjoyable. However, group work sometimes created misunderstanding and losing focus on the assigned tasks. The research also showed that there are interrelationships between spoken and written language in classroom. Although the students were to practise speaking English, they used written language in the process.

\subsection{Changes in EFL in Vietnam}

Lewis (1996), as discussed in Chapter 1, considered that Vietnamese learners had few chances to use English outside class. Eight years have passed since Lewis discussed English learning in Vietnam and the situation has not been much improved. Although the students in this research had more chances to speak English to foreigners and Vietnamese, they had to do their best to seek these limited opportunities. Lewis (1996, pp. 40-41) also pointed out:

In a language class, cooperation may mean that the students have to wait while one student struggles to find the right word, or the students have to work in groups to complete language tasks and explain a difficult point to a fellow student.

The audio transcriptions of this research showed that the students did not struggle long to find the right words as they collaborated in learning vocabulary. Peers were helpful in explaining word meanings and forms as well as providing the words needed. The tasks could have affected the ways the students in the current research were interacting. It could be that, in the long pauses of the unassisted group work, the students were 
thinking about the words but it appeared that they were more likely to be referring to the artifacts as the videos showed (see Chapter 9).

Although the students in the current research learned English for different motives (section 11.2, Chapter 11), the majority chose English as a major at university because of societal factors including employment, communication, and the integration of Vietnam into the world. The current finding regarding the motives for English learning in Vietnam blended with similar findings by Denham (1997), Do (1999), Gayle (1994), McKay (1999) and Mydans (1995). This indicates that there has been little change in Vietnam regarding motives for learning English in the last decade.

Out of the classroom, the students in this research practised learning English on their own. They learned English vocabulary, read English materials, and sought chances to use English. In their individual learning, they learned the language as practice and revision of skills. In this sense, the students had histories of learning to bring into the discussions in the classroom. When taking part in the group work, the students could have shared ideas and learned new ideas or they simply practised English speaking skills. Fox (2001) criticised neo-Vygotskian constructivism for emphasising teaching as the shared construction of knowledge because this risks ignoring the extent to which learning depends on independent practice. In this research, the students' responses in the interviews (section 11.3, Chapter 11) suggest that these EFL learners did practise language skills by themselves in learning another language. 


\subsection{Implications}

There are several implications regarding group work processes, the use of classroom artifacts, especially, the textbook and the inclusion of the target culture into the language classroom teaching and learning that can be drawn from the findings of the current study.

In sociocultural theory, oral language has a mediational role in the learning process, which was demonstrated in the audio transcription of the current study. The classroom environment therefore should provide the students with opportunities to engage in social interaction. A combination of classroom and broader social contexts would give the students more realistic situations for using the language for interaction. The interactive environment to make such opportunities available includes pair work, group work, English speaking clubs and social projects. In Vietnam, learning English is largely restricted to the language environment in the classroom. In addition, classroom teachers usually have to work with more than 40 students in a class at university. The current research showed that it is possible for peers from the same class or different classes to study together in groups. However, the students should be instructed about how to collaborate to make the most of their time in group work.

The data from current study (Chapter 6) also suggest that there should be measures taken to increase the target language utterances of students in group work. One way to do this is to reduce the size of the group so that students can have more time and turns. Another way is to increase the time allowance for group work. Designing group work is a sensitive task which requires the classroom teacher to anticipate what roles the 
members should play. To enhance interaction in group work, a classroom with moveable chairs would provide the students with a more comfortable setting.

The friendship among the students in the research led to a tendency to have off-theassigned-topic moments (Chapters 6 and 7). However, it still contributed to positive feelings to the group learning, such as the feelings of belonging, or of being more natural and cheerful (Chapter 10). The senior student in the current research was a new member in the class. If the students are to study with more advanced students who have known the students for enough time, it is anticipated that there will be more positive effects when the assistance from the advanced students is provided because their relationship with the students would probably become less hierarchical. However, it is possible that more advanced students would be seen as having less authority.

The senior student in this research was not provided with any special instructions but she did her best to help other members in the groups. When doing this, she used up lots of turns and sometimes asked yes-no questions. As a result, the students sometimes provided "yes" or "no" answers and short responses (see the sequences of the assisted group in Chapter 7). Assisting other members in group work is a skill. Thus, the skill should be taught and learned by the more knowledgeable peer so that his/her assistance would be more effective. Gillies (2003) found that when teachers are trained to use specific communication skills during cooperative-interactional learning conditions, they engage in more mediated-learning interactions, ask more questions, and make fewer disciplinary comments than teachers who have been trained to implement cooperative group work in a cooperative condition only. 
The students in the unassisted groups used more Vietnamese and combined Vietnamese and English (Chapters 6, 7 and 8). EFL students need to be exposed to more cognitively demanding tasks in English, such as task planning and vocabulary explanation. Leontiev (1981) proposed what he termed progressive approaches in psychology and methodology that focus on thinking in a foreign language. He pointed out, "The more multifaceted the mental activity attained by the learner with the help of a foreign language, the more fluent and adequate will be the process of communication in that language" (p. 109). In this sense, the students need to be exposed to a variety of situations in which they can realise their meanings in conversation with native speakers or situations in which native speakers produce the language. The involvement in English-speaking activities will help trigger the cognitive process and the use of English at the same time.

Chapter 7 showed that there was confusion among the students in the unassisted groups when they were expected to understand what to do. The data suggest that there should be clear statements about the required tasks and procedures. When the students fully understand the task in English, they tend to rely less on the first language. If there are new words in a task in the second language, the words need predicting and explaining.

With regard to the textbook, the students in this research when using a textbook designed by foreign authors found some situations and topics foreign to them (Chapters 5 and Chapter 9). For a textbook to be used across cultures, there should be some notice of social and cultural differences. Some brief introduction and notes on the situations for each activity will help facilitate the mediational learning process among the learners. In addition, the classroom teacher's explanations before each activity will help 

for a group of students, a native speaking student and non-native speaking students if they are learning a second/foreign language.

Regarding research methodology, a combination of classroom observation and the investigation of the wider community offers insights in the learning process of the learners in formal and informal settings. As for different methods of data collection used in this research, the audio recording obtained the richest data, which revealed the natural speech of the students when they were working in groups. Video-tapes showed images of group work when they were not heard on the audio tapes, for example, students' non-verbal communication and artifact use. It provided a complementary method of data collection to the audio recording. Journals captured the students' reflections and provided structured responses to the research questions. Although the students could choose to use English or Vietnamese to write in the journal entries, most of them preferred to use English. Writing a journal helped the students in this particular study to practise using English. However, the interview was more flexible than the journals in probing the students' views of group work. The interview also helped to investigate the support of the wider community of Vietnamese society and culture in the process of learning English. Triangulation was to strengthen the robustness of the data. No single data collection method could fully reveal the processes of group interactions in this research.

\subsection{Areas for Further Research}

The following areas merit further investigation from a sociocultural theoretical perspective. First of all, sociocultural theory emphasises the mediational roles of the 
more knowledgeable peers in learning. The current research did not focus on what the senior student learned. However, because social interaction results in learning for both parties, it would provide insights if a further study could investigate what a more advanced student learns from assisting other students. Secondly, sociocultural theory research usually analyses the processes of learning with more capable peers (different from teachers) who are not trained. Would a senior student trained in sociocultural theory use different group work processes if this research were replicated? Thirdly, the current research was conducted with all Vietnamese students. Do groups of students from different cultures produce the same group interaction processes? Fourthly, although sociocultural theory recognises the role of artifacts, it is primarily interested in the role of speech in learning and development. The data from the current research show that the students relied on the textbook to think of responses to the imagined situations and to construct and share understanding with other group members. Can learners learn by themselves by reading textbooks? What roles do textbooks play in knowledge construction? Fifthly, with regard to first language use, the current research provided evidence of the use of and assistance from the first language in learning English. Would learners also use the first language in the tasks that they are familiar with or tasks with no new vocabulary? Lastly, under what circumstances would understanding shared and gained through group interaction be retained for later use?

In terms of EFL pedagogy, the following areas might benefit EFL teachers and learners if they receive further research attention: 1) The qualities of utterances produced to be examined in more detail, for example, the length or number of words (if the students use English only), and the content of each utterance, 2) In what circumstances the words learned through participating in group work are retained for later use, and 3) 
There are other aspects of learning English in addition to the acquisition of vocabulary for example; grammar, pronunciation, syntax and pragmatics.

\subsection{Conclusions}

This research used sociocultural theory as a conceptual framework. Sociocultural theory allowed the study to look at the group, rather than the individual, processes of learning and, particularly, to examine the characteristics of the zone of possible learning known as the ZPD in two different contexts of unassisted and assisted peer groups. Differences were observed in both the processes and outcomes of group discussions. In general, both types of groups were able to complete the language tasks as required; however, the presence of the more capable student in one kind of group created 1) different roles among peers, 2) different mediational processes, 3) different use of languages, 4) different use of artifacts, and 5) different feelings among the students during the group work.

The findings of the current study show that the roles of peers varied in the different contexts of group work. The students in both kinds of groups used oral language to negotiate what to do with a language task, to share understanding and to help each other with English words. Although the students were expected to practise oral English, Vietnamese played the mediational role in the process of planning a language task and explaining new words in English in the unassisted peer groups. The more knowledgeable peer virtually eliminated the use of the first language in the assisted peer groups. The interaction pattern in the unassisted peer groups was irregular whereas the interaction in the peer groups with the senior student was like a teacher-led situation 
with an IEF exchange and was dyadic between the senior student and each member of the groups. While the assisted peer groups were more efficient and task-focused in discussing the assigned topics, the unassisted peer groups experienced confusion and had more trouble getting started. In both types of groups, the group processes were interdependent: participants constructed their knowledge not only on the basis of their existing knowledge, but also on the understanding and knowledge of other members. Within a group, peers directed each other's attention to the new steps of the task by verbal and non-verbal interaction, through talking, sharing, or raising a question, and through pointing at an artifact or showing agreement by nodding.

The findings of the current study also show that students used written artifacts, mainly the textbook and the instructions on the blackboard, in oral interactions. The textbook was a mediational written artifact. It determined the structures of students' participation, guided their discourse and generated their thoughts in response to the questions it posed. The unassisted peer groups were working directly with the textbook whereas the unassisted peer groups were working through the interpretations of the senior student who was guided by the textbook herself. The unassisted peer groups relied more on the instructions on the blackboard to carry out the language tasks. In the assisted peer groups, the senior peer's explanations of the required tasks helped the students understand what to do.

Group work created feelings of both enjoyment and stress. However, the students in both kinds of groups enjoyed the group work rather than felt stressed. Some students reported the feelings of being natural in the unassisted peer groups, and some reported the feelings of being dependent on the senior student in the assisted peer groups. The 
students reported the enjoyment of learning new ideas, sharing ideas with peers and improving their language skills. Some reported stress due to participating in group work and using English.

In this research, Vietnamese culture was vividly reflected. When learning another language, the students reproduced and reconstructed the knowledge of their first culture. Their emotions shown in oral interaction were also related to Vietnamese cultural practices and beliefs. Outside the classroom, the students sought opportunities to use and learn the target language.

As discussed in Chapter 4, the gaps in research literature within a sociocultural framework included the roles of peers and how peers worked in different contexts, the mediation between oral interaction and written language, emotions and cognition and emotion and culture. The current research explored these relations. The data showed that the roles of peers were more complex than the presence of a more knowledgeable peer would suggest. One student's comment illustrates the benefits of the assisted group work:

Without the senior student, we did not know how to start a conversation. We did not reach a conclusion. We went around the topic. We focused only on one point and ignored difficult questions. (26-I) [Translation from Vietnamese.] 


\section{References}

Ageyev, V. (2003). Vygotsky's concept of social mediation in cultural context. Paper presented at the American Educational Research Association Annual Conference, April 21-25, Chicago.

Anderson, G. (1998). Fundamentals of educational research. London: Falmer Press.

Anton, M. (1999). The discourse of a learner-centered classroom: Sociocultural perspectives on teacher-learner interaction in the second language classroom. The Modern Language Journal, 83(3), 303-18.

Anton, M., \& DiCamilla, F. (1998). Socio-cognitive functions of L1 collaborative interaction in the L2 classroom. Canadian Modern Language Review, 54(3), 314-342.

Arievitch, I. M. (2002). Gal'perin's contribution to activity theory: A potential for an integrated view of development and learning? Paper presented at the 5 th Congress of ISCRAT, June 18-22, Amsterdam.

Bakhtin, M. M. (1981). Discourse in the novel (M. Holquist \& C. Emerson, Trans.). In M. Holquist (Ed.), The dialogic imagination: Four essays. Austin: University of Texas Press.

Bakhtin, M. M. (1986). Speech genres and other late essays (V. W. McGee, Trans.). Austin: University of Texas Press.

Barnard, R. (2000). Non English speaking background learners in the mainstream classroom: A New Zealand case study. Unpublished doctoral thesis, University of Southampton.

Barnard, R. (2002). Peer tutoring in the primary classroom: A sociocultural interpretation of classroom interaction. New Zealand Journal of Educational Studies, 37(1), 57-72.

Bell, J. (1999). Doing your research project (3rd Ed.). Buckingham: Open University Press.

Blanck, G. (1990). Vygotsky: The man and his cause. In L. C. Moll (Ed.), Vygotsky and education: Instructional implications and applications of sociohistorical psychology (pp. 31-58). New York: Cambridge University Press.

Bozhovick, L. (1977). The concept of the cultural historical development of the mind and its prospects. Soviet Psychology, 16(1), 5-22.

Brooks, F. B., \& Donato, R. (1994). Vygotskyan approaches to understanding foreign language learner discourse during communicative tasks. Hispania, 77(2), 262274. 
Brown, A. L., Metz, K. E., \& Campione, J. C. (1996). Social interaction and individual understanding in a community of learners: The influence of Piaget and Vygotsky. In A. Tryphon \& J. Voneche (Eds.), Piaget-Vygotsky: The social genesis of thought (pp.145-170). Hove: Psychology Press.

Brown, M. (1989). What are the qualities of good research? In F. H. Hultgren \& D. L. Coomer (Eds.), Alternative modes of inquiry in home economics research (pp. 257-297). Peoria: Glencoe.

Candlin, C., \& Mercer, N. (2001). Introduction. In C. Candlin \& N. Mercer (Eds.), English language teaching in its social context (pp. 1-10). New York: Routledge.

Celik, M. (2003). Teaching vocabulary through code-mixing. ELT Journal, 57(4), 361369.

Cobb, P. (1998). Learning from distributed theories of intelligence. Mind, Culture, and Activity, 5(3), 187-204.

Cole, M. (1996). Cultural psychology: A once and future discipline. Cambridge: Harvard University Press.

Cole, M. (1998). Can cultural psychology help us think about diversity? Mind, Culture, and Activity, 5(4), 291-304.

Cole, M., \& Engeström, Y. (1993). A cultural-historical approach to distributed cognition. In G. Salomon (Ed.), Distributed cognitions: Psychological and eductional considerations (pp. 1-46). New York: Cambrige University Press.

Cole, M., John-Steiner, V., Scribner, S., \& Souberman, E. (Eds.). (1978). Mind in society: The development of higher psychological processes. Cambridge: Harvard University Press.

Coll, C., \& Onrubia, J. (1997). The construction of shared meanings in the classroom: Joint activity and semiotic mechanisms in the monitoring performed by teacher and learners. In C. Coll \& D. Edwards (Eds.), Teaching, learning and classroom discourse (pp. 49-66). Madrid: Fundacion Infancia y Aprendizaje.

Confrey, J. (1995). How compatible are radical constructivism, sociocultural approaches, and social constructivism? In L. Steffe \& J. Gale (Eds.), Constructivism in education (pp. 185-226). New Jersey: Lawrence Erlbaum Associates.

Cook, V. (2001). Using the first language in the classroom. Canadian Modern Language Review, 57(3), 402-423.

Creswell, J. W. (1994). Research design: Qualitative and quantitative approaches. Thousand Oaks: SAGE. 
Dalton, S. S., \& Tharp, R. (2002). Standards for pedagogy: Research, theory and practice. In G. Wells \& G. Claxton (Eds.), Learning for the 21st century (pp. 181-194). Malden: Blackwell.

D'Andrade, R. (1984). Cultural meaning systems. In R. A. Shweder \& R. A. Levine (Eds.), Culture theory: Essays on mind, self, and emotion (pp. 88-122). New York: Cambridge University Press.

Daniels, H. (Ed.). (1993). Charting the agenda: Educational activity after Vygotsky. London: Routledge.

Daniels , H. (2001). Vygotsky and pedagogy. London: Routledge Falmer.

Dean, A. (1994). Instinctual affective forces in the internalisation process: Contribution of Hand Loewald. Human Development, 37(1), 42-57.

De Guerrero, M., \& Villamil, O. (1994). Social-cognitive dimensions of interaction in L2 peer revision. The Modern Language Journal, 78(4), 484-496.

Denham, P. (1992). English in Vietnam. World Englishes, 11(1), 61-69.

Denham, P. (1997). Eight Autumns in Hanoi. In B. Kenny \& W. Savage (Eds.), Language and development: Teachers in a changing world (pp. 193-207). New York: Longman.

Denscombe, M. (1999). The good research guide. Buckingham: Open University Press.

Denzin, N. K. (1970). The research act in sociology: A theoretical introduction to sociological method. London: Butterworth.

Denzin, N. K., \& Lincoln, Y. (1994). Handbook of qualitative research. Thousand Oaks: Sage.

Dixon-Krauss, L. (Ed.). (1996). Vygotsky in the classroom. New York: Longman.

Do, H. T. (1996). Foreign language education policy in Vietnam (1975-1995): The emergence of English and its impact on higher education. Unpublished doctoral thesis, University of Southern California, Los Angeles.

Do, H. T. (1999). Foreign language education policy in Vietnam: The emergence of English and its impact on higher education. Paper presented at the Fourth International Conference on Language and Development, October 13-15, Hanoi, Vietnam.

Donato, R. (1988). Beyond group: A psycholinguistic rationale for collective activity in second-language learning. Unpublished doctoral dissertation, University of Delaware. 
Donato, R. (1994). Collective scaffolding in second language learning. In J. Lantolf \& G. Appel (Eds.), Vygotskian approaches to second language acquisition (pp. 3354). Norwrood: Ablex Press.

Donato, R., \& Bonnie, A. (1992). Discourse perspectives on formal instruction. Language Awareness, 1, 73-89.

Duong, T. H. O. (2003). Teaching and learning oral presentation for learners of English in universities in Vietnam and New Zealand: A comparative study. Unpublished doctoral thesis, Victoria University of Wellington, Wellington.

Durkheim, E. (1956). Education and sociology (S. Fox, Trans.). Illinois: The Free Press.

Ellis, G. (1995). Teaching and learning styles in Vietnam: Lessons for Australian educators. Journal of Vietnamese Studies, 8, 9-16.

Engeström, Y. (1991). Overcoming the encapsulation of school learning. Learning and Instruction, 1(3), 243-259.

Engeström, Y., Miettinen, R., \& Punamäki, R.-L. (Eds.). (1999). Perspectives on Activity Theory. New York: Cambridge University Press.

Ernest, P. (1995). The one and the many. In L. Steffe \& J. Gale (Eds.), Constructivism in education (pp. 459-486). New Jersey: Lawrence Erlbaum Associates.

Eyring, J., \& Silverman, M. (1996). Conducting teacher training workshops in Vietnam: Imposition or exchange. CATESOL Journal, 9(2), 133-142.

Firestone, W. (1993). Alternative arguments for generalizing from data as applied to qualitative research. Educational Researcher, 22(4), 16-23.

Flowerdew, L. (1998). A cultural perspective on group work. ELT Journal, 52(4), 323329.

Forman, E. A., \& Cazden, C. B. (1985). Exploring Vygotskian perspectives in education: The cognitive value of peer interaction. In J. V. Wertsch (Ed.), Culture, communication, and cognition: Vygotskian perspectives (pp. 323-347). Cambridge: Cambridge University Press.

Fosnot, C. T. (1996). Constructivism: A psychological theory of learning. In C. T. Fosnot (Ed.), Constructivism: Theory, perspectives and practice (pp. 8-33). New York: Teachers College, Columbia University.

Fosnot, C. T. (Ed.). (1996). Constructivism: Theory, perspectives and practice. New York: Teachers College, Columbia University.

Fox, R. (2001). Constructivism examined. Oxford Review of Education, 27(1), 23-35. 
Frawley, W., \& Lantolf, J. (1984). Second language discourse: A Vygotskian perspective. Applied Linguistics, 6(1), 19-44.

Gagne, R. M. (1965). The conditions of learning. New York: Holt, Rinehart \& Winston.

Gayle, K. (1994). Vietnam. In G. Butzbatch \& T. O'Donnell \& M. Kupetz (Eds.), Teaching EFL outside the United States. (3rd ed., pp. 73-77). Alexandria: TESOL Publisher.

Geertz, C. (1993). The interpretation of cultures: Selected essays. London: Fontana.

Gibbons, P. (2001). Learning a new register in a second language. In C. Candlin \& N. Mercer (Eds.), English language teaching in its social context (pp. 258-270). London: Routledge.

Gibbons, P. (2003). Mediating language learning: Teacher interactions with ESL students in a content-based classroom. TESOL Quarterly, 37(2), 247-273.

Gillies, R. (2003). The effects of training in specific interactional skills on teacher and student discourses during cooperative learning. Paper presented at the New Zealand Association of Research in Education and the Australian Association of Research in Education Joint Conference: Risks and Dilemmas, 29 Nov-3 Dec, Hyatt Regency Auckland and the University of Auckland.

Goldstein, L. (1999). The relational zone: The role of caring relationships in the coconstruction of mind. American Educational Research Journal, 36(3), 647-673.

Granott, N. (1993). Patterns of interaction in the co-construction of knowledge: Separate minds, joint effort, and weird creatures. In R. F. Wozniak, K. (Ed.), Development context (pp. 183-210). London: Lawrence Erlbaum Associates.

Gudykunst, W. B. (1998). Bridging differences: Effective intergroup communication (3rd ed.). Thousand Oaks: Sage.

Hill, C., Cummings, M., \& van Aalst, J. (2003). Activity theory as a framework for analyzing participation within a knowledge building community. Paper presented at the Annual Meeting of the American Educational Research Association, April 21-15, Chicago.

Hitchcock, G., \& Hughes, D. (1989). Research and the teacher. London: Routledge.

Hofstede, G. (1997). Cultures and organisations: Software of the mind. (2nd ed.). London: McGrawHill.

Holzman, L. H. (1996). Pragmatism and dialectical materialism in language development. In H. Daniels (Ed.), An introduction to Vygotsky (pp. 75-94). London: Routledge. 
Ingram, E. (1975). Psychology and language learning (Vol. 2). New York: Oxford University Press.

Jennings, C., \& Di, X. (1996). Collaborative learning and thinking: The Vygotskian approach. In L. Dixon-Krauss (Ed.), Vygotsky in the classroom (pp. 77-91). New York: Longman.

Joe, A., Nation, P., \& Newton, J. (1996). Vocabulary learning and speaking activities. English Teaching Forum, 34(1), 2-13.

John-Steiner, V., (2000). Creative collaboration. New York: Oxford University Press.

John-Steiner, V., \& Mahn, H. (1996). Sociocultural approaches to learning and development: A Vygotskian framework. Educational Psychologist, 31(3/4), 191206.

Joravsky, D. (1987). L. S. Vygotskii: The muffled deity of Soviet psychology. In M. Ash \& W. Woodward (Eds.), Psychology in twentieth-century thought and society (pp. 189-212). New York: Cambridge University Press.

Jordan, B. J. (2003). Professional development making a difference for children: Coconstructing understandings in early childhood centres. Unpublished doctoral thesis, Massey University, Palmerston North.

Kelep-Malpo, K. D. (2003). Gender and school leadership in the Papua New Guinea. Unpublished doctoral thesis, Victoria University of Wellington, New Zealand.

Kellerman, E., \& van Hoof, A. M. (in press). Manual accents. IRAL, 41.

Knight, T. (1996). Learning vocabulary through shared tasks. The Language Teacher, 20, 24-29.

Kobayashi, M. (2003). The role of peer support in ESL students' accomplishment of oral academic tasks. The Canadian Modern Language Review, 59(3), 337-368.

Kozulin, A. (1998). Psychological tools: A sociocultural approach to education. Cambridge: Harvard University Press.

Kozulin, A. (2003). Psychological tools and mediated learning. In A. Kozulin \& B. Gindis \& V. S. Ageyev \& S. M. Miller (Eds.), Vygotsky's educational theory in cultural context (pp. 15-38). New York: Cambridge University Press.

Kramsch, C. (1990). What is foreign language learning research? In B. VanPatten \& J. F. Lees (Eds.), Second language acquisition-Foreign language learning (pp. 2733). Clevedon: Multilingual Matters Ltd.

Kramsch, C., \& Sullivan, P. (1996). Appropriate pedagogy. ELT Journal, 50(3), 199212. 
Krashen, S. (1981). Second language acquisition and second language learning. Oxford: Pergamon.

Labov, W. (1972). Sociolinguistic patterns. Philadelphia: University of Pennsylvania Press.

Lantolf, J. (2000a). Second language learning as a mediated process. Language Teaching, 33(2), 79-96.

Lantolf, J. (Ed.). (2000b). Sociocultural theory and second language learning. New York: Oxford.

Lantolf, J. (2002). Sociocultural theory and second language acquisition. In R. Kaplan (Ed.), The Oxford handbook of applied linguistics (pp. 104-114). New York: Oxford University Press.

Lantolf, J., \& Appel, G. (Eds.). (1994). Vygotskian approaches to second language research. New Jersey: Ablex Publishing.

Larsen, J. (1999). Analyzing participation frameworks in kindergarten writing activity: The role of the overhearer in learning to write. Written Communication, 16(2), 225-227.

Lave, J., \& Wenger, E. (1991). Situated learning: Legitimate peripheral participation. New York: Cambridge University Press.

Lave, J., \& Wenger, E. (1996). Practice, person, social world. In H. Daniels (Ed.), An introduction to Vygotsky (pp. 143-150). London: Routledge.

Le, H. H. P. (2002a). Problems of student participation. Teacher's Edition, 9, 54-55.

Le, H. H. P. (2002b). Sociocultural theory and teaching English as a foreign language. Paper presented at the 5th ISCRAT congress, June 18-22, Amsterdam, Holland.

Le, H. H. P. (2003a). The mediational role of language teachers in sociocultural theory. English Teaching Forum, 41(3), 31-35.

Le, H. H. P. (2003b). What does a more knowledgeable peer mean? A sociocultural analysis of group interaction in a Vietnamese classroom. Paper presented at the NZARE/AARE Joint New Zealand and Australia conference: Risks and Dilemmas, Nov 29-Dec 03, The University of Auckland, New Zealand.

Lee, C., \& Smagorinsky, P. (2000). Introduction: Constructing meaning through collaborative inquiry. In C. Lee \& P. Smagorinsky (Eds.), Vygotskian perspectives on literacy research (pp. 1-18). Cambridge: Cambridge University Press.

Leontiev, A. A. (1981). Psychology and the language learning process. Oxford: Pergamon Press. 
Levine, J. (1993). Learning English as an additional language in multilingual classrooms. In H. Daniels (Ed.), Charting the agenda: Educational activity after Vygotsky (pp. 190-216). London: Routledge.

Lewis, M. (1996). Teaching English in Vietnam: A teacher training handbook. Auckland: Institute of Language Teaching and Learning.

Lightbown, P., \& Spada, N. (1999). How languages are learned. Oxford: Oxford University Press.

Lincoln, Y. S., \& Guba, E. G. (1985). Naturalistic inquiry. Thousand Oaks, CA: Sage.

Linfors, J. W. (1999). Children's inquiry: Using language to make sense of the world. New York: Teacher College, Columbia University.

Long, M., \& Porter, P. (1985). Group work, interlanguage talk, and second language acquisition. TESOL Quarterly, 19(2), 207-228.

Luria, A. R. (1928). The problem of the cultural development of the child. Journal of Genetic Psychology, 35, 493-506.

Luria, A. R. (1979). The making of mind. Cambridge: Harvard University Press.

Mahn, H. (1997). Dialogue journals: Perspectives of second language learners in a Vygotskian theoretical framework. Unpublished doctoral dissertation, University of Mexico, Albuquerque.

Mahn, H., \& John-Steiner, V. (2002). The gift of confidence: A Vygotskian view of emotions. In G. Wells \& G. Claxton (Eds.), Learning for life in the 21th century: Sociocultural perspectives on the future of education (pp. 46-58). Oxford: Blackwell.

Manning, B., \& Payne, B. (1993). A Vygotskian-based theory of teacher cognition: Toward the acquisition of mental reflection and self-regulation. Teaching and Teacher Education, 9(4), 361-371.

Matusov, E. (2001). Vygotskij's theory of human development and new approaches to education. In N. J. Smelser \& P. B. Balters (Eds.), International encyclopedia of the social and behavioral sciences (pp. 16339-16343). Oxford: Elsevier Science Ltd.

Mayan, M. J. (2001). An introduction to qualitative methods: A training module for students and professionals. Alberta: International Institute for Qualitative Methodology.

McCafferty, S. (2002). Gesture and creating zones of proximal development for second language learning. The Modern Language Journal, 86(2), 192-203. 
McCook, F. (1998). A longitudinal study of responses to in-service teacher education by Vietnamese English language teachers. Unpublished master's thesis, Massey University, Wellington.

McDonald, G., Kidman, J., \& Clarke, V. (1991). Junior school study: A study of classrooms containing five-year-olds. Wellington: New Zealand Council of Educational Research.

McKay, S. (1999). A look at Vietnam and Cuba. TESOL Matters, 1(6), $1 \& 7$.

Meacham, J. (1996). Mind, society, and racism. Human Development, 39(5), 301-306.

Mercer, N. (1992). Talk for teaching-and-learning. In K. Norman (Ed.), Thinking voices: The work of the national oracy project (pp. 204-215). London: NCC Enterprises Ltd.

Mercer, N. (1995). The guided construction of knowledge. Clevedon: Multilingual Matters Ltd.

Mercer, N. (2000). The analysis of talk as data in educational settings. Paper presented at the 3rd International conference on sociocultural research, Campinas, Brazil.

Mercer, N. (2001). Language for teaching a language. In C. Candlin \& N. Mercer (Eds.), English teaching in its social context (pp. 243-257). London: Routledge.

Mishler, E. G. (2000). Validation in inquiry-guided research: The role of exemplars in narrative studies. In B. M. Brizuela \& J. P. Stewart \& R. G. Carrillo \& J. G. Berger (Eds.), Acts of inquiry in qualitative research (pp. 119-146). Cambridge: Harvard Educational Review.

Mistry, J. (2001). Narratives in cultural socialization. Human Development, 44(6), 337339.

Mitchell, R., \& Myles, F. (1998). Second language learning theory. London: Arnold.

Moll, L. C. (1989). Teaching second language students: A Vygotskian Perspective. In J. Donna \& R. Duane (Eds.), Richness in writing: Empowering ESL students (pp. 55-69). New York: Longman.

Moll, L. C. (Ed.). (1990). Vygotsky and education: Instructional implications and applications of sociohistorical psychology. Cambridge: Cambridge University Press.

Moll, L. C. (2000). Inspired by Vygotsky: Ethnographic experiments in education. In C. D. Lee \& P. Smagorinsky (Eds.), Vygotskian perspectives on literacy research: Constructing meaning through collaborative inquiry (pp. 256-268). Cambridge: Cambridge University Press. 
Moll, L. C., Amanti, C., Neff, D., \& Gonzalez, N. (1992). Funds of knowledge for teaching: Using a qualitative approach to connect homes and classrooms. Theory Into Practice, XXXI(2), 132-141.

Moll, L. C., \& Whitmore, K. (1993). Vygotsky in classroom practice: Moving from individual transmission to social transaction. In E. A. Forman \& N. Minick \& C. A. Stone (Eds.), Contexts for learning: Sociocultural dynamics in children's development (pp. 19-42). New York: Oxford University Press.

Mydans, S. (1995). Vietnam speaks English with an eager accent. New York Times, CXLIV (50,054), 16.

Nation, I. S. P. (1990). Teaching and learning vocabulary. Massachusetts: Heinle \& Heinle.

Nation, I. S. P. (2001). Learning vocabulary in another language. New York: Cambridge University Press.

Negueruela, E., Lantolf, J., Jordan, R. S., \& Gelabert, J. (2003). The 'private function' of gesture in second language speaking activity: A study of motion verbs and gesturing in English and Spanish. Paper presented at the Annual Meeting of American Educational Research Association, 15-21 April, Chicago.

Newman, F., \& Holzman, L. (1993). Lev Vygotsky: Revolutionary scientist. London: Routledge.

Newton, J. (2001). Options for vocabulary learning through communication tasks. ELT Journal, 55(1), 30-37.

Nguyen, B. (2003). The design and use of English language teaching materials in Vietnamese secondary schools. Unpublished doctoral thesis, Victoria University of Wellington, Wellington.

Nguyen, H. Q. (1994). Tieng Viet hien dai. Hanoi: Trung Tam Bien Soan Tu Dien Bach Khoa Viet Nam.

Nunan, D. (1992). Research methods in language learning. New York: Cambridge University Press.

Nuthall, G., \& Alton-Lee, A. (1990). Research on teaching and learning: Thirty years of change. The Elementary School Journal, 90(5), 547-570.

Nyikos, M., \& Hashimoto, R. (1997). Constructivist theory applied to collaborative learning in teacher education: In search of ZPD. The Modern Language Journal, 81(4), 506-517.

Ohta, A. (1995). Applying sociocultural theory to an analysis of learner discourse: Learner-learner collaborative interaction in the zone of proximal development. Issues in Applied Linguistics, 6(2), 93-121. 
Olmedo, I. (2003). Language mediation among emergent bilingual children. Linguistics and Education, 14(2), 143-162.

Palincsar, A. S., \& Brown, A. L. (1984). Reciprocal teaching of comprehensionfostering and comprehension-monitoring activities. Cognition and Instruction, 1, 117-175.

Panofsky, C. (2003). The relations of learning and student social class. In A. Kozulin \& B. Gindis \& V. S. Ageyev \& S. M. Miller (Eds.), Vygotsky's educational theory in cultural context (pp. 411-431). New York: Cambridge University Press.

Park, C. (2000). Learning style preferences of Southeast Asian students. Urban Education, 35(5), 245-268.

Pham, M. T. (2000). Nghe thuat ung xu cua nguoi Viet. Hanoi: Nha Xuat Ban Van Hoa Thong Tin.

Piaget, J. (1977). Equilibration of cognitive structures. New York: Basic Books.

Prawat, R. (1999). Social constructivism and the process-content distinction as viewed by Vygotsky and the pragmatists. Mind, Culture, and Activity, 6(4), 255-273.

Ramsey, G., \& Rees-Parnal, H. (1989). Well-Spoken. Essex: Longman.

Rogoff, B. (1998). Cognition as a collaborative process. In W. Damon \& D. Kuhn \& R. Siegler (Eds.), Handbook of child psychology (5th ed., Vol. 2, pp. 679-744). New York: John Wiley \& Sons, Inc.

Rogoff, B. (2003). The cultural nature of human development. New York: Oxford University Press.

Schwarz, B., \& Hershkowitz, R. (2001). Production and transformation of computer artifacts: Toward construction of meaning in mathematics. Mind, Culture, and Activity, 8(3), 20-267.

Sfard, A., \& Kieran, C. (2001). Cognition as communication: Rethinking learning-bytalking through multi-faceted analysis of students' mathematical interactions. Mind, Culture, and Activity, 8(1), 42-76.

Silka, L., \& Tip, J. (1994). Empowering the silent ranks: The Southeast Asian experience. American Journal of Community Psychology, 22(4), 479-512.

Skilbeck, M. (Ed.). (1970). John Dewey. London: Collier-Macmillan Limited.

Skinner, B. F. (1953). Science and human behaviour. New York: Free Press.

Stake, R. (1995). The art of case study research. Thousand Oaks: Sage. 
Stetsenko, A., \& Arievitch, I. (1997). Constructing and deconstructing the self: Comparing post-Vygotskian and discourse-based versions of social constructivism. Mind, Culture, and Activity, 4(3), 159-172.

Stone, A. (1998). The metaphor of scaffolding: Its utility for the field of learning disabilities. Journal of Learning Disabilities, 31(4), 344-379.

Storch, N. (2002). Patterns of interaction in ESL pair work. Language Learning, 52(1), 119-158.

Sullivan, P. (1996). Sociocultural influences on classroom interactional styles. TESOL Journal, 6(1), 32-34.

Sullivan, P. (2000). Playfulness as mediation in communicative language teaching. In J. Lantolf (Ed.), Sociocultural theory and second language learning (pp. 97-114). New York: Oxford.

Swain, M. (1995). Three functions of output in second language learning: Studies in honour of H.G. Widdowson. In G. Cook \& B. Seidlhofer (Eds.), Principle and practice in applied linguistics (pp. 125-144). Oxford: Oxford University Press.

Swain, M. (2000). The output hypothesis and beyond: Mediating acquisition through collaborative dialogue. In J. Lantolf (Ed.), Sociocultural theory and second language learning (pp. 97-114). New York: Oxford University Press.

Swain, M., \& Lapkin, S. (1998). Interaction and second language learning: Two adolescent French immersion students working together. Modern Language Journal, 82(2), 320-337.

Tharp, R., \& Gallimore, R. (1988). Rousing mind to life. New York: Cambridge University Press.

Toulmin, S. (1999). Knowledge as shared procedures. In Y. Engeström \& R. Miettinen \& R.-L. Punamaki (Eds.), Perspectives on activity theory (pp. 53-64). New York: Cambridge University Press.

Tran, T. T. B. (2003). The storybook reading practice of first graders in Vietnamese families: Six case studies. Unpublished master's thesis, Dunedin College of Education, Dunedin.

Tudge, J. R. H., Winterhoff, P. A., \& Hogan, D. M. (1996). The cognitive consequences of collaborative problem solving with and without feedback. Child Development, 67(6), 2892-2909.

University curriculum book for teaching English, (1996-1997). Hue: Hue University.

Van Boxtel, C., van der Linden, J., \& Kanselaar, G. (2000). The use of textbooks as a tool during collaborative physics learning. Journal of Experimental Education, 69(1), 57-76. 
Van der Veer, R., \& Ijzendoorn, M. H. (1985). Vygotsky's theory of the higher psychological processes: Some criticisms. Human Development, 28, 1-9.

Van der Veer, R., \& Valsiner, J. (1991). Understanding Vygotsky: A quest for synthesis. Oxford: Blackwell.

Van der Veer, R., \& Valsiner, J. (Eds.). (1994). The Vygotsky reader. Oxford: Blackwell.

Van Lier, L. (1996). Interaction in the language curriculum: Awareness, autonomy and authenticity. London: Longman.

Van Lier, L. (1998). Constraints and resources in classroom talk: Issues of equality and symmetry. In H. Byrnes (Ed.), Learning foreign and second languages: Perspectives in research and scholarship (pp. 157-182). New York: The Modern Language Association of America.

Van Lier, L. (2000). From input to affordance: Social-interactive learning from an ecological perspective. In J. Lantolf (Ed.), Sociocultural theory and second language learning (pp. 245-261). New York: Oxford University Press.

Villamil, O., \& De Guerrero, M. C. (1996). Peer revision in the L2 classroom: Sociocognitive activities, mediating strategies, and aspects of social behaviour. Journal of Second Language Writing, 5, 51-75.

Vygotsky, L. S. (1962). Thought and language (E. Hanfmann \& G. Vakar, Trans.). Cambridge: Massachusetts Institute of Technology.

Vygotsky, L. S. (1978). Mind in society: The development of higher psychological processes. Cambridge: Harvard University Press.

Vygotsky, L. S. (1981). The instrumental method in psychology. In J. Wertsch (Ed.), The concept of activity in Soviet psychology (pp. 134-143). Armonk: Sharpe.

Vygotsky, L. S. (1986). Thought and language (A. Kozulin, Trans.). Cambridge: The MIT Press.

Vygotsky, L. S. (1987). Thinking and speech (N. Minick, Trans.). In R. Rieber \& A. Carton (Eds.), The collected works of L. S. Vygotsky: Problems of general psychology (Vol. 1, pp. 39-243). New York: Plenum Press.

Wartofsky, M. (1979). Models: Representation and scientific understanding. Dordrecht: Reidel.

Wells, G. (1993). Working with a teacher in the zone of proximal development: Action research on the learning and teaching of science. Journal of the Society for Accelerative Learning and Teaching, 18(1/2), 127-222. 
Wells, G. (1998). Using L1 to master L2: A response to Anton \& DiCamilla's Sociocognitive function of $\mathrm{L} 1$ collaborative interaction in the $\mathrm{L} 2$ classroom. The Canadian Modern Language Review, 54(3), 343-353.

Wells, G. (1999). Dialogic inquiry. New York: Cambridge University Press.

Wells, G. (2002). The role of dialogue in activity theory. Mind, Culture, and Activity, 9(1), 43-66.

Wenger, E. (1998). Communities of practice: Learning, meaning, and identity. Cambridge: Cambridge University Press.

Wertsch, J. (1998). Mind as action. New York: Oxford University Press.

Wertsch, J., Del Rio, P., \& Alvarez, A. (Eds.). (1995). Sociocultural studies of mind. New York: Cambridge University Press.

Wertsch, J., \& Tulviste, P. (1992). L. S. Vygotsky and contemporary developmental psychology. Developmental Psychology, 28(4), 548-557.

Wertsch, J. (1985). Vygotsky and the social formation of mind. Cambridge: Harvard University Press.

Wertsch, J., \& Rogoff, B. (1984). Editors' notes. In B. Rogoff \& J. Wertsch (Eds.), Children's learning in the "zone of proximal development". (pp. 1-6). San Francisco: Jossey-Bass, Inc.

Wertsch, J., \& Toma, C. (1995). Discourse and learning in the classroom: A sociocultural approach. In L. Steffe \& J. Gale (Eds.), Constructivism in education (pp. 159-174). New Jersey: Lawrence Erlbaum Associates.

Wink, J., \& Putney, L. (2002). A vision of Vygotsky. Arlington: Allyn and Bacon.

Wood, D., Bruner, J. S., \& Ross, G. (1976). The role of tutoring in problem-solving. Journal of Child Psychology and Child Psychiatry, 17, 89-100. 


\section{Appendix A}

\section{Ethics Application Letter to an Institution in Vietnam}

[Note: This letter was translated into Vietnamese.]

Huong Hoai Pham Le, PhD student

School of Education

Victoria University of Wellington

New Zealand

Dear Sirs and Madams,

I am Huong Hoai Pham Le. I am currently doing my $\mathrm{PhD}$ in education at Victoria University of Wellington. My research is to investigate neo-Vygotskian approaches in teaching English at university level in Vietnam with a focus on assisted versus unassisted group work in English speaking classes.

I would like to inform you of my research. I will invite two first- year English classes, two or three students from a more advanced class, and two teachers of the English department to participate in my research. I will give all the participants an information sheet of the research to explain its nature. I will also give them a consent form to ask for their consent to participate into my research in which they will be recorded, photographed, observed, and asked to keep diaries. When I get their consent, I will be observing these students in their classes during English speaking periods when there are group work activities in a four-month semester. For each observation, I will focus on two groups. One group of four first-year students will be assisted by a more advanced student and the other group of five first-year students will not. Subject to equipment availability and participants' consent, all the observations will be videotaped, their interaction in group work will be audio-recorded and transcribed for data processing. In addition, all of the students will be asked to keep diaries on what they learn from group work. This one-week diary entry would focus on vocabulary, functions of English used in discussion, concepts of a theme and their feelings when engaging in group work. At the end of the semester, all of the participants will be interviewed. The interviews which are expected to take about 20 minutes will be audio recorded. The research purpose is to improve the learning and teaching English in Vietnam. It has no other intentions that might do harm to the students or teachers.

The teachers and students at any time can withdraw from the research. There will not be any penalties if they do so. Students' grades will not be affected. If they would like to withdraw, all the data collected on them will be destroyed.

I would like to request your permission for access to observe one first year English class in two weeks of the second semester beginning late January 2002 and another in three months with audio and video tape recorders. I would like also to request your consent for me to interview and record interviews with the students participating in the research.

All the responses from the interviews or diaries will be put into a written report on a confidential basis. None of the research participants will be identified. All materials 
collected will be kept confidential. No other person besides me and my supervisors, Dr. Val Podmore and Dr. Geraldine McDonald, will see the audio, video and interviews recordings and diaries. The thesis will be submitted for marking to the School of Education and deposited in the Victoria University Library. It is intended that one or more articles will be submitted for publication in scholarly journals. Diaries and videos will be destroyed two years after the end of the project. I will send all the research participants a two-page summary of the research findings and will offer your university one copy of the thesis, subject to its being accepted by examiners, for use as a resource by our university and students.

The participation of the teachers and students is voluntary. There will not be penalties of any sort if they withdraw. If the students withdraw, their grades will not be affected and all materials collected on them will be destroyed. Audiotapes will be wiped but videotapes will be destroyed two years after the research completion. The video-tapes focus on group of students, so one single person's images cannot be taken out without destroying the whole tapes. However, I am willing to look at possible ways to blank out one person's identity on the videos if he/she withdraws.

If you have any questions or would like to receive further information about the project, please contact me at Lehuon@scs.vuw.ac.nz or my supervisors, Dr. Geraldine McDonald and Dr. Val Podmore at the School of Education at Victoria University, P O Box 600, Wellington, New Zealand, phone 64-4-463-5350.

I am looking forward to your reply. Thank you.

Yours sincerely,

Huong Hoai Pham Le 


\title{
Appendix B
}

Information Sheet for the Teacher/s Participating in a Study of Neo-Vygotskian Approaches in Teaching English as a Foreign Language at University Level in Vietnam: Assisted versus Unassisted Group Work in English Speaking Classes*

\author{
Dear colleague/s,
}

I am Huong Hoai Pham Le. I am currently doing my $\mathrm{PhD}$ in education at Victoria University of Wellington. My research is to investigate the learning and teaching English as a foreign language at university level in Vietnam in the framework of sociocultural approach developed by Vygotsky and his followers.

I am inviting you to participate in my research. I will not interfere with your teaching process but will be observing your class during speaking periods when there are group work activities in a four-month semester. Each observation is expected to last 20 minutes every week. For each observation, I will focus on two groups, one is assisted by a more advanced student and the other is not. Each group has five members. Only students will be observed during the study, not the teachers. The camera will be put in the position that focuses on these two groups. With this position of the camera, if you happen to get into the camera area, your face will be visible. An information sheet of the research and a consent form will be sent to each individual student participating in the research. Their engagement in group work will be video-taped with a focus on their facial expressions and gestures. Their speech in group interaction will be audio-taped and transcribed for data processing. All of the students will be interviewed at the end of the semester. The interviews which are expected to last 20 minutes will be audiorecorded. In addition, the students will be also asked to keep diaries on what they learn from group work. This one-week diary entry would focus on some vocabulary, functions of English used in discussion, concepts of a theme and their feelings when engaging in group work. The students will write freely on their reflections on group work study regarding these categories. Papers with guiding headings for diaries will be given to the students every week for their diary entry.

All the data from observations, interviews or diaries will be put into a written report on a confidential basis. None of the research participants will be identified. No other person besides me and my supervisors, Dr. Val Podmore and Dr. Geraldine McDonald, will see the audio, video, and interview recordings and diaries.

The research participants will be encouraged to ask for feedback about the process of the research personally. I will answer any inquiries from them during my four- month field trip in Vietnam to help clarify what I am doing and why I need the information from them. Diaries and videos will be destroyed two years after the end of the project. The thesis will be submitted for marking to the School of Education and deposited in the Victoria University Library. It is intended that one or more articles will be submitted for publication in scholarly journals. I will offer the research participants a two-page research findings and your university one copy of the thesis, subject to its being accepted by examiners, for use as a resource by the university and students. 
Please let know whenever you prefer your class not to be observed. You always have the right to withdraw. And so do the students. If the students withdraw they will not be asked to join the groups under observation and all data collection on them will be destroyed. If they withdraw, their grades will not be affected. If you have any questions or would like to receive further information about the project, please contact me at Lehuon@scs.vuw.ac.nz or my supervisors, Dr. Geraldine McDonald and Dr. Val Podmore at the School of Education at Victoria University,

P O Box 600, Wellington, New Zealand, phone 64-4-463-5350.

Yours sincerely,

Huong Hoai Pham Le

* This was the initial general topic of the thesis. 


\section{Appendix C}

Consent to Participation in Research: Teacher

\section{A Study of Neo-Vygotskian Approaches in Teaching English as a Foreign Language at University Level in Vietnam: Assisted versus Unassisted Group Work in Speaking Classes}

I have been given and have understood an explanation about this research project. I would agree that Huong Le may observe my class during group work time. I consent to my classroom being recorded by audio and video tapes and being photographed. I understand that I will not be recorded or identified under any circumstance. The individual students have the right to withdraw at any time without penalties of any sort. Their grades and class participation will not be affected. If the students withdraw, they will not be asked to join groups of students under observations and all the data collected on them will be destroyed. Their voice on the audio-tape will be wiped but their pictures on the videotape will not be erased until two years after the research completion. As the videotape focuses on a group of five members, one single image can not be taken out without destroying the whole videotape.

I understand that I can ask for feedback about the process of the research personally. I also understand the thesis will be submitted for marking to the School of Education and deposited in the Victoria University Library in New Zealand. A 2-page summary of the research findings will be sent to us and one copy of the thesis, subject to its being accepted by examiners, will be offered for use as a resource by the university and students in Vietnam.

I have read this letter and understood its terms. I am willing to take part in this research.
Name:
Signature:
Date: 


\section{Appendix D}

\section{Information Sheet for Students Participating in a Study of Neo-Vygotskian Approaches in Teaching English as a Foreign Language at University Level in Vietnam: Assisted versus Unassisted Group Work in English Speaking Classes}

[Note: This information sheet was translated into Vietnamese.]

\section{Dear student/s,}

I am Huong Hoai Pham Le. I am currently doing my $\mathrm{PhD}$ in education at Victoria University of Wellington. My research is to investigate the learning and teaching English as a foreign language at university level in Vietnam in the framework of sociocultural approach developed by Vygotsky and his followers.

I am inviting you to participate in my research. I will not interfere with your learning process but will be observing you during speaking periods when there are group work activities in a four-month semester. For each observation, I will focus on two groups, one is assisted by a more advanced student and the other is not. Each group has 5 members. All the observations will be video-taped and audio-recorded. Your engagement in group work will be video-taped with a focus on your facial expressions, and gestures. Your interaction in group work will be audio-taped and transcribed for data processing. You will be interviewed at the end of the semester. The interviews which are expected to last 20 minutes will be audio- recorded. You will be also asked to keep a diary on what you learn from group work. This one-week diary entry would focus on some vocabulary, functions of English used in discussion, concepts of a theme and your feelings when you engage in group work. You can write freely your reflections on group work study regarding these categories. A paper with guiding headings will be given to you every week for your diary entry.

All of your responses from interviews or diaries will be put into a written report on a confidential basis. You will not be identified under any circumstance. All materials collected will be kept confidential. No other person besides me and my supervisors, Dr. Val Podmore and Dr. Geraldine McDonald, will see the diaries or video or interview recordings. The thesis will be submitted for marking to the School of Education and deposited in the Victoria University Library. It is intended that one or more articles will be submitted for publication in scholarly journals.

You will be encouraged to ask for feedback about the process of the research personally. I will answer any inquiries from you during my four- month field trip in Vietnam to help clarify what I am doing and why I need the information from you. Diaries, audiotapes and videos will be destroyed two years after the end of the project. I will offer our university one copy of the thesis, subject to its being accepted by examiners, for use as a resource by the university and students. I will also send you a two-page summary of my research findings.

Your participation in this research is voluntary and not compulsory. If you do not consent, you will not be asked to join the groups under observation. There will be no 
penalties on your grades if you do not consent to participate or you withdraw. However, you are encouraged to participate to make the research possible.

If you would like to withdraw from the project, you may do so without question at any time before the data is analysed before 30 May 2002. Please let me know at the time. If you withdraw, you will not be asked to join the groups under observations when there are group work activities in the class and the data collected on you will be destroyed. Your voice on the audiotape will be wiped but your pictures on the videos will be wiped two years after the completion of the research. The videos focus on a group of five, so your pictures cannot be erased without destroying the whole tapes with pictures of other members. However, I would be willing to look at possible ways to blank out your face on the videos if you withdraw. If you have any questions or would like to receive further information about the project, please contact me at Lehuon@scs.vuw.ac.nz or my supervisors, Dr. Geraldine McDonald and Dr. Val Podmore at the School of Education at Victoria University, P O Box 600, Wellington, New Zealand, phone 64-4-463-5350.

Yours truly,

Huong Hoai Pham Le 


\section{Appendix E}

\section{Consent to Participating in Research: Students}

\section{A Study of Neo-Vygotskian Approaches in Teaching English as a Foreign Language at University Level in Vietnam: Assisted Versus Unassisted Group Work in Speaking Classes}

[Note: This consent form was translated into Vietnamese.]

I have been given and have understood an explanation about this research project. I understand that my participation is voluntary and I might at any time withdraw myself or any information I have provided from this project before data collection and analysis are complete (30 May 2002) without having penalty of any sort. I understand that if I withdraw, my grades will not be affected and the data collected about me will be destroyed. The tape will be wiped but the video will be erased after the research completion because the tape has groups of students so a single picture cannot be blanked out without ruining the whole tape.

I know that the information provided by me in the diaries and interviews will be kept confidential and I will not be identified under any circumstance. I can ask for feedback about the process of the research personally. I also understand that all the responses will be destroyed two years after the project has been completed. The thesis will be submitted for marking to the School of Education and deposited in the Victoria University Library in New Zealand. A two-page summary of the research findings will be sent to the research participants and one copy of the thesis, subject to its being accepted by examiners, will be offered for use as a resource by the university and students in Vietnam.

I have read this letter and understood its terms. I am willing to take part in this research.

I agree (please tick in the appropriate box)

\begin{tabular}{|l|l|}
\hline To be observed in group work & \\
\hline To be audio-taped & \\
\hline To be videotaped & \\
\hline To be photographed & \\
\hline To keep a diary & \\
\hline To be interviewed & \\
\hline
\end{tabular}

Name:

Signature:

Date: 


\section{Appendix F}

\section{Journal Questions for Unassisted Students}

Name/Tên: -Date/Ngày:

Group discussion topic/activity: Chủ đề hoạt động/hoạt động:

\section{UNASSISTED STUDENT}

Please complete this journal in English or in Vietnamese and return to me tomorrow. I will see you in your class to pick up this journal. Thank you very much for your cooperation.

\section{CHO SINH VIÊN KHÔNG ĐƯợC TRỢ GIÚP}

Xin vui lòng viết nhật ký học tập này bằng tiếng Anh hay tiếng Việt. Tôi sẽ gặp bạn vào buổi học hôm sau để thu bản nhật ký học tập này.

1. Did you have an opportunity to speak in English in the group work today? If yes, what did you say? Please list as many things as you can.

Hôm nay bạn có cơ hội để nói tiếng Anh khi thảo luận trong nhóm không? Bạn đã nói về điều gì? Xin vui lòng viết lại những gì bạn đã nói.

2. If you didn't speak in the group work today, why not?

Nếu bạn không nói tiếng Anh trong thảo luận trong nhóm ngày hôm nay, xin giải thích vì sao bạn không nói?

3. Did the discussion in the group work today bring you new knowledge of English and other things? If yes, what was it? Please give as many examples as you can.

Thảo luận trong nhóm hôm nay có mang lại cho bạn những kiến thức mới về tiếng Anh cũng như các lãnh vực khác không? Những kiến thức đó là gì? Xin cho ví dụ cụ thể.

4. Were other group members helpful to you today? If yes, please explain and give examples.

Những thành viên trong nhóm có giúp bạn hay không? Nếu có, xin vui lòng giải thích và cho ví dụ cụ thể.

5. In your opinion are there any disadvantages in the group work today? Please explain and give examples.

Theo bạn hoạt động luyện nói theo nhóm ngày hôm nay có những bất thuận lợi nào không? Xin vui lòng cho ví dụ cụ thể.

6. Did you enjoy the group work today? If yes, what in particular did you enjoy? 
Bạn có thích thảo luận theo nhóm hôm nay không? Nếu có, xin vui lòng giải thích bạn thích những gì trong thảo luận theo nhóm?

7. Do you think that some students in your group did not enjoy the group work today? Can you explain why they did not like the group work?

Bạn có nghĩ là một số sinh viên trong nhóm của bạn không thích họat động theo nhóm ngày hôm nay không? Xin vui lòng giải thích tại sao họ không thích họat động theo nhóm ngày hôm nay?

8. Did you find the group work today stressful? Please explain if yes or no.

Bạn có thấy căng thẳng trong khi thảo luận theo nhóm hôm nay không? Xin giải thích vì sao có hay không? 


\section{Appendix G}

\section{Journal Questions: Assisted Groups}

Name/Tên:

-Date/Ngày:

Group discussion topic/activity: Chủ đề hoạt động/hoạt động:

\section{ASSISTED STUDENT}

Please complete this journal in English or in Vietnamese and return to me tomorrow. I will see you in your class to pick up this journal. Thank you very much for your cooperation.

\section{CHO SINH VIÊN ĐƯợC TRỢ GIÚP}

Xin vui lòng viết nhật ký học tập này bằng tiếng Anh hay tiếng Việt. Tôi sẽ gặp bạn vào buổi học hôm sau để thu bản nhật ký học tập này.

1. Did you have an opportunity to speak in English in the group work today? If yes, what did you say?

Hôm nay bạn có cơ hội để nói tiếng Anh khi thảo luận trong nhóm không? Bạn đã nói về điều gì? Xin vui lòng viết lại những gì bạn đã nói.

2. If you didn't speak in the group work today, why not?

Nếu bạn không nói tiếng Anh trong thảo luận trong nhóm ngày hôm nay, xin giải thích vì sao bạn không nói?

3. Did the discussion in the group work today bring you new knowledge of English and other things? If yes, what was it? Please give as many examples as you can.

Thảo luận trong nhóm hôm nay có mang lại cho bạn những kiến thức mới về tiếng Anh cũng như các lãnh vực khác không? Những kiến thức đó là gì? Xin cho ví dụ cụ thể.

4. Were other group members helpful to you today? If yes, please explain and give examples.

Những thành viên trong nhóm có giúp bạn hay không? Nếu có, xin vui lòng giải thích và cho ví dụ cụ thể.

5. In your opinion are there any disadvantages in the group work today? Please explain and give examples.

Theo bạn hoạt động luyện nói theo nhóm ngày hôm nay có những bất thuận lợi nào không? Xin vui lòng cho ví dụ cụ thể.

6. Was the senior student helpful to you? If yes, please explain and give examples. Sinh viên từ lớp cao hơn có giúp bạn không? Nếu có xin vui lòng giải thích và cho ví dụ cụ thể. 
7. What do you think are the disadvantages of having the senior student in the group work today? Please explain and give examples.

Theo bạn thì những bất thuận lợi khi có một sinh viên từ lớp cao hơn trong họat động theo nhóm ngày hôm nay là gì?

8. Did you enjoy group work today? If yes, what in particular did you enjoy?

Bạn có thích thảo luận theo nhóm hôm nay không? Nếu có, xin vui lòng giải thích bạn thích những gì trong thảo luận theo nhóm?

9. Do you think that some students in your group did not enjoy the group work today? Can you explain why they did not like the group work?

Bạn có nghĩ là một số sinh viên trong nhóm của bạn không thích họat động theo nhóm ngày hôm nay không? Xin vui lòng giải thích tại sao họ không thích họat động theo nhóm ngày hôm nay?

10. Did you find group work stressful? Please explain if yes or no.

Bạn có thấy căng thẳng trong khi thảo luận theo nhóm hôm nay không? Xin giải thích vì sao có hay không? 


\section{Appendix I}

\section{Interview Questions}

1. Do you study vocabulary at home? If yes, how?

Bạn có học từ vựng ở nhà không? Nếu có bạn học bằng cách nào?

2. Do you read at home? In what languages? And what do you usually read? Bạn có đọc nhiều ở nhà không? Bạn đọc bằng ngôn ngữ nào? Bạn thường đọc những gì?

3. Do you find the materials yourself? Were there recommendations from others? Bạn có tự tìm kiếm tài liệu để đọc không? Có ai giới thiệu cho bạn không?

4. Do you like to practise speaking English when you are by yourself? Bạn có thích luyện tập nói tiếng Anh một mình không?

5. What learning resources are there in your home? What learning resources are there in your classroom? Which learning aids do you find particularly helpful? Ở nhà bạn có những phương tiện học tập nào? Ở lớp bạn có những phương tiện học tập nào? Những phương tiện học tập nào là hữu ích đối với bạn?

6. Do you think it is a good idea to learn from other students? Please explain yes or no.

Bạn có nghĩ là học hỏi từ các sinh viên cùng khóa là một ý kiến hay không? Xin giải thích lý do tại sao?

7. Do you like to have a senior student to help you in group work? Why or why not?

Bạn có thích có một sinh viên trình độ cao hơn giúp bạn khi học theo nhóm không? Tại sao có/không?

8. In what ways was your experience in the group work with the senior student different from without her?

Học theo nhóm có sinh viên trình độ cao hơn và học theo nhóm với các sinh viên cùng lớp khác nhau như thế nào?

9. Would you like to have more group work?

Bạn có muốn có thêm luyện tập theo nhóm không?

10. Have you ever used English with foreigners? If yes, in what contexts? Bạn có bao giờ sử dụng tiếng Anh với người nước ngòai chưa? Nếu có, trong bối cảnh nào?

11. Have you ever used English with your friends? If yes, in what contexts? Bạn có bao giờ sử dụng tiếng Anh với bạn bè chưa? Nếu có, trong bối cảnh nào?

12. Would you consider speaking English out of the classroom important? Why or why not? 
Bạn có nghĩ là nói tiếng Anh ngòai bối cảnh lớp học là quan trọng không? Tại sao có/không?

13. Why are you learning English?

Tại sao bạn lại học tiếng Anh?

14. Who was influential in guiding you in majoring in English?

$\mathrm{Ai}$ đã ảnh hưởng đến việc chọn ngành học tiếng Anh của bạn?

15. Do your family members speak English? Do you speak English to your family members? If yes, in what contexts?

Gia đình bạn có ai nói tiếng Anh không? Bạn có nói tiếng Anh với các thành viên trong gia đình bạn không? Nếu có, trong bối cảnh nào? 


\section{Appendix J}

\section{Summary of Categories and Coding}

\begin{tabular}{|c|c|c|c|c|}
\hline $\begin{array}{l}\text { Data } \\
\text { source }\end{array}$ & Main categories & Subcategories & Definitions & Coding \\
\hline \multirow{8}{*}{$\begin{array}{l}\text { Audio- } \\
\text { transc- } \\
\text { ription }\end{array}$} & \multirow{4}{*}{ Utterances } & English utterances & Completely in English & T-EU \\
\hline & & Vietnamese utterances & Completely in Vietnamese & T-VU \\
\hline & & $\begin{array}{l}\text { English and Vietnamese } \\
\text { utterances }\end{array}$ & $\begin{array}{l}\text { Combined language: English and } \\
\text { Vietnamese }\end{array}$ & T-EVU \\
\hline & & Off-the-assigned-topic & Irrelevant to the requirement of the task & T-OT \\
\hline & \multirow{3}{*}{$\begin{array}{l}\text { Mediated } \\
\text { interaction }\end{array}$} & $\begin{array}{l}\text { Negotiation of } \\
\text { understanding }\end{array}$ & $\begin{array}{l}\text { Sharing understanding of the topics } \\
\text { under discussion }\end{array}$ & T-NU \\
\hline & & $\begin{array}{l}\text { Negotiation of task } \\
\text { planning and } \\
\text { procedures }\end{array}$ & $\begin{array}{l}\text { Joint-working on task requirement and } \\
\text { procedures }\end{array}$ & T-NTP \\
\hline & & $\begin{array}{l}\text { Negotiation of word } \\
\text { meanings and forms }\end{array}$ & $\begin{array}{l}\text { Shared understanding of word meanings } \\
\text { or forms }\end{array}$ & T-NW \\
\hline & Laughter & & Episodes of laughter on audio tapes & $\begin{array}{l}\text { T- } \\
\mathrm{L} 1 \ldots \mathrm{Ln}\end{array}$ \\
\hline \multirow{4}{*}{$\begin{array}{l}\text { Video } \\
\text { tapes }\end{array}$} & $\begin{array}{l}\text { Non verbal } \\
\text { interaction }\end{array}$ & & Hand and head movement, eye contact & V-NVC \\
\hline & Use of Artifacts & & $\begin{array}{l}\text { Students used the textbook, and other } \\
\text { classroom artifacts. }\end{array}$ & V-A \\
\hline & $\begin{array}{l}\text { Starting } \\
\text { snapshots }\end{array}$ & & $\begin{array}{l}\text { The discussion started with the first two } \\
\text { students to speak. }\end{array}$ & V-SS \\
\hline & $\begin{array}{l}\text { Ending } \\
\text { snapshots }\end{array}$ & & $\begin{array}{l}\text { When the students finished the } \\
\text { discussion and the last student to end the } \\
\text { discussion. }\end{array}$ & V-ES \\
\hline \multirow[t]{11}{*}{ Journals } & $\begin{array}{l}\text { Opportunities } \\
\text { to speak }\end{array}$ & & Reports on opportunities to speak. & $\mathrm{J}-\mathrm{O}$ \\
\hline & \multirow{4}{*}{ Vocabulary } & New words & $\begin{array}{l}\text { Reports on the words that the students } \\
\text { learned. }\end{array}$ & J-NW \\
\hline & & $\begin{array}{l}\text { Joint-learning of } \\
\text { vocabulary }\end{array}$ & $\begin{array}{l}\text { The students helped each others with } \\
\text { words. }\end{array}$ & J-JLW \\
\hline & & $\begin{array}{l}\text { Collective memory of } \\
\text { vocabulary }\end{array}$ & $\begin{array}{l}\text { Reports on how students helped each } \\
\text { others to remember and recall words. }\end{array}$ & J-CMW \\
\hline & & $\begin{array}{l}\text { Unsuccessful learning } \\
\text { of vocabulary }\end{array}$ & & J-ULV \\
\hline & \multirow{4}{*}{$\begin{array}{l}\text { Discussion of } \\
\text { topic }\end{array}$} & $\begin{array}{l}\text { Students' knowledge of } \\
\text { the topics }\end{array}$ & $\begin{array}{l}\text { Reports on the familiarity and } \\
\text { knowledge of the students to the topics }\end{array}$ & J-TK \\
\hline & & $\begin{array}{l}\text { Students' evaluation of } \\
\text { the topics }\end{array}$ & $\begin{array}{l}\text { The students liked to discuss the topics } \\
\text { or not }\end{array}$ & J-TI \\
\hline & & Effective discussion & Reports on successful discussion & J-ED \\
\hline & & Ineffective discussion & Reports on unsuccessful discussion & $\mathrm{J}-\mathrm{ID}$ \\
\hline & \multirow{2}{*}{$\begin{array}{l}\text { Students' } \\
\text { feelings }\end{array}$} & Enjoyment & $\begin{array}{l}\text { Reports on positive feelings. The } \\
\text { students enjoyed the discussion. }\end{array}$ & J-E \\
\hline & & Stress & $\begin{array}{l}\text { Reports on negative feelings. The } \\
\text { students were stressed. }\end{array}$ & $\mathrm{J}-\mathrm{S}$ \\
\hline
\end{tabular}




\begin{tabular}{|c|c|c|c|c|}
\hline & $\begin{array}{l}\text { Students' } \\
\text { perception of } \\
\text { the role of the } \\
\text { senior student }\end{array}$ & & $\begin{array}{l}\text { How the senior student helped with the } \\
\text { group work. }\end{array}$ & J-PS \\
\hline \multirow{17}{*}{$\begin{array}{l}\text { Inter- } \\
\text { views }\end{array}$} & $\begin{array}{l}\text { Vocabulary } \\
\text { learning at } \\
\text { home }\end{array}$ & & $\begin{array}{l}\text { How the students learned vocabulary at } \\
\text { home }\end{array}$ & I-VLH \\
\hline & Materials & $\begin{array}{l}\text { Materials for reading at } \\
\text { home }\end{array}$ & $\begin{array}{l}\text { Reports on the availability and access to } \\
\text { materials at home }\end{array}$ & I-MRH \\
\hline & $\begin{array}{l}\text { Learning } \\
\text { resources at } \\
\text { home }\end{array}$ & & $\begin{array}{l}\text { What the students had for learning at } \\
\text { home }\end{array}$ & I-LTH \\
\hline & $\begin{array}{l}\text { Learning } \\
\text { resources in } \\
\text { class }\end{array}$ & & Artifacts in class & I-TIC \\
\hline & $\begin{array}{l}\text { Learning with } \\
\text { classmates }\end{array}$ & & $\begin{array}{l}\text { Students' evaluation and perceptions of } \\
\text { learning with classmates }\end{array}$ & I-LC \\
\hline & $\begin{array}{l}\text { Learning in } \\
\text { group work }\end{array}$ & & $\begin{array}{l}\text { Students' evaluation and perceptions of } \\
\text { group work }\end{array}$ & I-GW \\
\hline & $\begin{array}{l}\text { Learning with } \\
\text { the SS }\end{array}$ & & $\begin{array}{l}\text { Students' evaluation and perceptions of } \\
\text { learning with the senior student }\end{array}$ & I-LSS \\
\hline & $\begin{array}{l}\text { Vocabulary in } \\
\text { group work }\end{array}$ & & $\begin{array}{l}\text { Students talked about the experience } \\
\text { about learning vocabulary in group work } \\
\text { under observation }\end{array}$ & I-VGW \\
\hline & $\begin{array}{l}\text { Topic } \\
\text { discussion in } \\
\text { group work }\end{array}$ & & $\begin{array}{l}\text { Students talked about the experience } \\
\text { about topic discussion in group work } \\
\text { under observation }\end{array}$ & I-TGW \\
\hline & \multirow{2}{*}{ Feelings } & Positive feelings & & I-PA \\
\hline & & Negative feelings & & I-NA \\
\hline & \multirow{4}{*}{$\begin{array}{l}\text { English out of } \\
\text { classroom } \\
\text { context }\end{array}$} & With foreigners & & I-EFo \\
\hline & & With friends & & I-EFr \\
\hline & & $\begin{array}{l}\text { With family members } \\
\text { Evaluation of the }\end{array}$ & & I-EF \\
\hline & & $\begin{array}{l}\text { importance of speaking } \\
\text { English out of the } \\
\text { classroom context }\end{array}$ & & I-IEP \\
\hline & $\begin{array}{l}\text { Motives to learn } \\
\text { English }\end{array}$ & $\begin{array}{l}\text { Intrinsic and extrinsic } \\
\text { motives }\end{array}$ & & I-RLE \\
\hline & & $\begin{array}{l}\text { Factors to affect the } \\
\text { choice of English as a } \\
\text { major at university }\end{array}$ & & I-FEC \\
\hline
\end{tabular}




\section{Appendix K}

\section{A Sample of Audio-Transcription}

Topic: Anger. Activity: What makes you angry? How do you show your anger? Think about your feelings, and then write your answers in the chart below. The textbook provides a list of imagined situations.

\section{Unassisted Group}

$01 \mathrm{~S} 1 \mathrm{Mau}$ len. [Hurry up!]

02 S2 Hoi, hoi cau mot [Try question number one]. When a visitor to your home accidentally breaks your favourite vase, I think I will try to buy another one later.

03 S3 When a visitor to my home accidentally breaks my favourite vase, I will try to make the visitor feel comfortable when they have break carelessly.

04 S4 Yes, if a visitor to my home accidentally breaks my favourite vase although I am very sad but I try to make the visitor not to make my visitor feel uncomfortable

05 S3 Uncomfortable?

06 S4 Khong duoc thoai mai (Explanation in Vietnamese the meaning of the word uncomfortable)

(2 seconds pause)

07 S2 When a visitor to my home accidentally breaks my favourite vase, I think that may be a big problem, so we must think about the problem and have best solutions to choose to solve when we have made them.

08 S1 I think you need to answer one two three four five. (5 seconds pause)

09 S2 Question two: Your child runs across the road without looking at the traffic, and nearly gets run over. What will do you do Ha?

$10 \mathrm{~S} 4$ If my child runs across the road without looking at the traffic, and nearly gets run over, I will say it is very lucky for my child and I will say with my child more carefully and tell my child that you could

11 S1 You should

12 S4 You should be more carefully when you run across the road.

(2 seconds pause)

13 S5 What will you do when someone pushes in front of you in a queue in a busy shop?

(12 seconds pause)

(Inaudible)

$\mathrm{T}$ (Teacher's reminder) Make sure everyone is talking.

14 S2 Accidentally-Accident a

$15 \mathrm{~S} 1$ Someone bumps into your car when you are waiting at the traffic lights, what will you do?

$16 \mathrm{~S} 4 \mathrm{I}$ think if someone hits your motorbike.

17 S2 The same one hits your motorbike.

18 S3 OK, no problem. 
19 S2 No problem?

20 S5 I don't think so. If someone bumps into my car when waiting at the traffic lights, I will recommend him give me some money.

(Laughter)

21 S3 To repair my car.

22 S1 If your car doesn't break.

23 S5 If my car does not break I will wait his apologize.

$24 \mathrm{~S} 1$ Your favourite television program is cancelled because a sports programme goes on too long.

25 S4 In this situation,

26 S2 If I am watching the TV program but it is cancelled by a sports program I will ask them to

27 S3 No, TV program

$28 \mathrm{~S} 2$ What a pity. How about you?

(2 seconds pause)

29 S3 A friend telephones to say they can't come to see you tonight as planned because they've been invited to a party, what would you do?

30 S1 I will say it is a pity I am very sorry for your not inviting not visiting my party but I will say many interesting things at my party for him. And you?

31 S2 Your husband/wife/girlfriend/boyfriend forgets your birthday.

32 S4 For me, for this question, I will say with my friend I am sorry for my apology, I forgot I have a big calendar. Quen [I forgot.] Xin loi [I am sorry.]

(2 seconds pause)

33 S4 I don't have anything to remind them to remember my birthday

34 S1 But I think this friend knows your birthday but he can't come because he is busy. I ask you what would you do if it happens.

35 S4 If that happens I will be very sad.

36 S1 Someone leans out of your car. Lean (Pronounce the word again.)

$37 \mathrm{~S} 2$ Lean

38 S4 And makes a rude sign at me, shouting that I am a bad driver.

39 S5 I think he is impolite.

40 S1 Impolite?

41 S5 Do you think he is mad?

(Laughter)

42 S3 Yes, but if it is not a wrong answer.

43 S4 In the park, someone's dog jumps up at you and covers your coat with mud, what would you do?

44 S5 I will be very angry and shout ah ah.

(Laughter)

45 S3 No I will say the boss of the dog and recommend he should teach the dog.

The dog, (Laughter)

46 S5 Do you understand it is the dog!

$47 \mathrm{~S} 3$ You tell the boss of the ...

(5 seconds pause)

48 S2 The next question

$49 \mathrm{~S} 1$ In a restaurant, the waiter ignores you and begins to serve other tables although you were sitting first.

$50 \mathrm{~S} 3$ I will not come to that restaurant again.

$51 \mathrm{~S} 1$ But you need to eat.

52 S3 I will go to another restaurant. 
$53 \mathrm{~S} 1$ But you don't have money you don't have much time.

54 S3 I think I will come to another restaurant and there are many restaurants to choose. 


\section{Assisted Group}

01 SS OK, now let's start. You have the books, right? Yeah, we will do part 4. Can you read out the first situation?

02 S1 The first one? A visitor to your home accidentally breaks your favourite vase.

03 SS What will do you then in this situation?

04 S1 Maybe I will get angry.

05 SS Angry? Why?

06 S1 Because it's my favourite vase. Maybe I can't buy another one.

07 SS So how about you?

08 S2 I will get angry. It is my favourite vase.

09 SS You mean you will get a little angry. Why do you get a little bit angry?

10 S2 Because it's my favourite vase.

11 SS And that means you can't buy another one. Will you get angry also?

12 S3 I don't think so. Because he is my guest. You can't be angry with him.

13 SS So if you don't get angry what will you do then? What is your behaviour then?

14 S3 I think it is ok.

15 SS Will you smile? (Laugher)

$16 \mathrm{~S} 3$ Yes.

17 SS Will you tell him to buy you another one?

$18 \mathrm{~S} 3$ Yes.

19 SS Yes?

20 S3 No.

21 SS You mean you just smile? How about you?

22 S4 I agree with Hung. If he breaks my favourite vase, I can buy another one.

23 SS Do you get angry with the guest? Just smile? So which behaviour do you like the most?

24 S1/S2 Hung's behaviour.

25 SS Just smile. Don't get angry. How about the second situation? Can you read out the second situation?

26 S2 Your child runs across the road without looking at the traffic, and nearly gets run over.

27 SS Do you understand clearly the point? What will you do then?

28 S1 First I will shout at the children.

29 SS Shout at your child?

30 S1 Don't cross the road...I think he will not have permit to cross the road. We shouldn't permit the child to cross the road because they are small.

31 SS Will you agree with her? Do you have any other behaviours?

32 S2 I will advise him to stay in his room and then I will advise him not to run across the road...

33 SS Without looking at the traffic? Do you think it is a dangerous thing?

So how about you?

34 S3 I agree with you.

35 SS And you?

36 S3 Me too.

37 SS You should have your own opinions. Suppose you are a father and your child runs across the road. Please speak out your opinions, what will you do as a father?

38 S3 I think I will have someone to help my child. 
39 SS What do you mean?

40 S3 Someone to help my child.

41 SS So usually the father in the family gets angry and beats the child if the child does the wrong things. Will you beat the child?

42 S3 Yes.

43 SS As a mother will do you so?

44 S2 Maybe I will beat.

45 SS So which is the best thing to deal with the child? Advise him? Or beat him?

46 S4 Should not beat the child. He is too small. I will keep the child in the room for two hours.

$47 \mathrm{SS}$ In this case, what is your favourite behaviour? The behaviour that you like most?

48 S3 I think I advise my child.

49 SS Will you keep him in the room for two hours like her? What about the third situation? Can you read out?

50 S2 Someone pushes in front of you in a queue in a busy shop.

51 S4 Queue? What does it mean?

52 SS Whenever you buy something, you will have to stand in a row to wait for the other person to buy something and then it's your turn next. Suppose that you have to wait for your turn, you know, when you buy something, for example, the ticket. Do you understand?

$53 \mathrm{~S} 4$ Yes.

54 SS What will you do then? Will you get angry with the person?

$55 \mathrm{~S} 3$ Of course.

56 SS What else will you react?

57 S3 I think I talk with him about his...

58 SS Behaviour. Will you shout at him?

59 S1 I don't think so. I think he sees my face.

(3 seconds pause) (Laughter)

60 SS He sees your face and he knows that you get angry? So, how about you?

61 S2 If it was by accident, and he apologizes, I will smile and forgive him If he ignores, I will shout at him.

62 SS In this situation, what will you react? What is your behaviour?

63 S4 I agree with Dung. If he apologizes, I will forgive him. I think he should not push the other person. I mean maybe he pushes in front of me by accident.

64 SS Do you all agree with her? Advise him to stand in a queue? Should not push the person? Do you think it is a good answer?

65 All Yes.

66 SS How about the next situation?

67 S3 Someone bumps into your car when you are waiting at the traffic lights. Do you understand everything?

68 SS So in this situation, what will you do then?

69 S1 I think I will say the person to be more careful.

70 SS Will you say so, like that?

71 S2 If it happens again, I will ask him to

72 SS To give you some money. (Laughter) (4 seconds pause)

73 S2 To repair the car

74 S4 I will tell him to be more careful

75 SS And you, will you ask him for some more money? 
$76 \mathrm{~S} 1$ No

77 SS As a boy, what will you do then? Usually, the boy is very angry. How about you?

78 S3 If he does not damage the car seriously.

$79 \mathrm{SS}$ In case he bumps into your car and makes a lot of damages to your car, what will you do then?

80 S3 I will be angry and I require him for money for repairing my car.

81 SS All of you will ask him for money?

82 All Yes, of course. (Laughter)

$83 \mathrm{SS}$ You want money. So the best behaviour is to ask for money. Let's move to the next situation.

$84 \mathrm{~S} 4$ Your favourite television programme is cancelled because a sports programme goes on too long.

85 SS So what do you think about this situation?

$86 \mathrm{~S} 1$ If I can't watch the program, I can go and read or listen to music so I will not...won't feel angry.

87 SS And you, what will you do in this situation? Will you wait for your favourite program? Or read some book or listen to music like her?

88 S2 Maybe I will listen to some music, write a letter to the editor.

89 SS That's great. Will you do like that?

90 S4 I think I will do something to wait for my favourite programme.

91 SS What about you?

$92 \mathrm{~S} 3$ I'll turn off the TV, I hate this programme.

93 SS Will you write a letter to the editor of programme like her? Write something to complain about the program. Do you think in this case writing a letter to complain is good?

94 S4 No.

95 SS Why do you think so?

96 S4 I think they change the program by accident- accidentally because the sports programs sometimes are live (pronounces the word as /liv/)

97 S1 Live (pronounces the word as /laiv/)

98 S4 You shouldn't get angry.

99 SS What is your decision then?

100 S2 A letter will make the editor to think about the program again.

101 SS How about the next situation?

$102 \mathrm{~S} 2 \mathrm{~A}$ friend telephones to say they can't come and see you tonight as planned because they've been invited to a party.

103 SS Will you be sad in this situation?

104 S1 Maybe I feel angry about this. Disappointed. Maybe I will change my plan. And make another plan. I will go out with another friend.

105 SS Will you do like her?

106 S4 At first, I feel very sad because I planned to do something, but I think I can do this with her another time.

107 SS That's all for today. 


\section{Appendix L}

\section{A Sample Interview Transcription with a Participant Student}

[This transcription was translated from Vietnamese into English by the researcher.]

I Good morning. How are you? What's your name?

N Good morning. My name is Nhung.

I Could we talk about how you learn English at home?

$\mathrm{N}$ Yes.

I Do you learn vocabulary at home?

N Yes, I do.

I How do you learn vocabulary at home?

$\mathrm{N}$ I write down words in Vietnamese on one side of a piece of paper and the equivalents in English on the other side of the paper. I also look up new words in a dictionary. I ask friends for difficult words. If my friends do not know, I ask teachers.

I That's really good. Do you read at home too?

N Yes, sometimes. I only read some short stories and newspapers in English, for example, Saigon Times. I also read the newspaper and stories in Vietnamese.

I Do you find the materials yourself or are they recommended?

N My friends recommend them to me. I also go to teachers' houses to borrow materials. There are many materials at my teachers' so I don't know which ones I should read.

I Do you like to practise speaking English when you are by yourself?

$\mathrm{N}$ Sometimes. If I use some words many times, I will be more confident to use them. I make up situations and plan what I have to say in these situations and speak English based on these situations.

I Very interesting! Do you have learning resources at home?

$\mathrm{N}$ Yes.

I What are they?

$\mathrm{N} \quad$ Cassette tapes, computer, books.

I How about in class?

$\mathrm{N}$ Cassette, tapes, and books and pictures. We studied grammar in the lab one time only.

I Which learning aids particularly help you with your English learning?

$\mathrm{N}$ Listening to tapes helps me a lot with my English learning, for example, pronunciation, communication skills, and quick reactions.

I Do you think it is a good idea to learn from other students?

$\mathrm{N} \quad$ Yes, I learned ideas from good students in the group.

I Do you like to have a senior student to help you in group work?

$\mathrm{N} \quad$ Yes, a senior student can help me with English learning and vocabulary. A senior student gave me suggestions and expanded the discussion. How was your group discussion with the senior student and without her different?

With the senior student, there was a unity in the group. Every one spoke English. However, sometimes it was easier to express oneself in Vietnamese rather than English. But the senior student knew how to help us to present our opinions. She gave us suggestions. 
Without the senior student, the group members sometimes used Vietnamese. Some students spoke more and some students spoke less. Sometimes I could not understand what they wanted to say because they did not know how to present their ideas. However, both kinds of groups were fun.

I Do you like to have more group work?

$\mathrm{N} \quad$ Yes, to improve my English. I want to speak and listen to English well to be more confident.

I Have you ever used English with foreigners?

$\mathrm{N}$ Yes, through letters with some Americans. I sometimes chat in English to learn English.

I Do you use English with your friends?

N Yes, but a little only, some common daily expressions.

I Do you think it is important to speak English out of the classroom context?

$\mathrm{N} \quad$ Yes, because it is different from the classroom. The discussions in class are limited to topics and some topics are compulsory and I don't know how to talk about them.

I Why are you learning English?

$\mathrm{N} \quad$ Because I have learned English since primary school and was better at English than other subjects. So I took the English university entrance exam as a major.

I Who was influential in your choice?

N Myself. English is my strong subject. I am not good at other subjects.

I Do your family members speak English?

N No, they don't.

I Do you speak English with them at home?

N No, of course not.

I Well, thank you very much for your time and your cooperation. 